César Yukishigue Kiyono

Método de Otimização Topológica aplicado ao Projeto de Sonotrodos para Transdutores Piezelétricos 
César Yukishigue Kiyono

\title{
Método de Otimização Topológica aplicado ao Projeto de Sonotrodos para Transdutores Piezelétricos
}

\author{
Dissertação apresentada à Escola Politécnica \\ da Universidade de São Paulo para obtenção \\ do Título de Mestre em Engenharia \\ Mecânica.
}


César Yukishigue Kiyono

\title{
Método de Otimização Topológica aplicado ao Projeto de Sonotrodos para Transdutores Piezelétricos
}

\author{
Dissertação apresentada à Escola Politécnica \\ da Universidade de São Paulo para obtenção \\ do Título de Mestre em Engenharia \\ Mecânica. \\ Área de concentração: \\ Engenharia de Controle e Automação \\ Mecânica \\ Orientador: \\ Prof. Dr. Emílio Carlos Nelli Silva
}


Este exemplar foi revisado e alterado em relação à versão original, sob responsabilidade única do autor e com a anuência de seu orientador.

São Paulo, de abril de 2008.

Assinatura do autor

Assinatura do orientador

FICHA CATALOGRÁFICA

Kiyono, César Yukishigue

Método de otimização topológica aplicado ao projeto de sonotrodos para transdutores piezelétricos / C.Y. Kiyono. -ed.rev. -- São Paulo, 2008.

$161 \mathrm{p}$.

Dissertação (Mestrado) - Escola Politécnica da Universidade de São Paulo. Departamento de Engenharia Mecatrônica e de Sistemas Mecânicos.

1. Sonotrodos 2. Ultrasonografia 3. Método dos elementos finitos 4. Método de otimização topológica 5. Transdutores piezelétricos I.Universidade de São Paulo. Escola Politécnica. Departamento de Engenharia Mecatrônica e de Sistemas Mecânicos II.t. 
Dedico este trabalho à minha noiva e à minha família, especialmente à minha mãe 


\section{Agradecimentos}

À Deus, por oferecer mais esta oportunidade para minha evolução.

Ao orientador, Prof. Dr. Emílio Carlos Nelli Silva, pela atenção, dedicação, paciência e empenho para a realização desse trabalho.

À FAPESP (Fundação de Amparo à Pesquisa do Estado de SP), pelo apoio financeiro deste trabalho, através da bolsa de mestrado.

Aos Prof. Dr. Júlio Cezar Adamowski e Prof. Dr. Alexandre Kawano, do Departamento de Engenharia Mecatrônica e de Sistemas Mecânicos da USP, e ao Prof. Dr. Eduardo Lenz Cardoso, do departamento de Engenharia Mecânica da UDESC, pelos comentários e dicas para o aprimoramento deste trabalho.

Aos meus pais, que sempre estiveram ao meu lado, fisica ou espiritualmente, dando todo tipo de suporte que eu necessitava, e por serem modelos de perseverança e disciplina, o que sempre me motivou a continuar. Aos meus tios Roberto e Elza, que cuidaram de mim em todos esses anos de faculdade enquanto estava em São Paulo. À minha noiva Patrícia, que esteve ao meu lado em todos os momentos dessa fase da minha vida.

Aos amigos de pesquisa Dr. Carbonari, Paulo Godoi, João, Sandro, Nakasone, Luís, Andres, Koga, Rogério, Wilfredo e ao alemão Christian, pelos bons momentos de discussões científicas e não científicas.

Aos amigos da Faculdade, Marcos, Erick, Carmelo, Marcão, Polastro, Rodrigo Alves, Fausto, Ramon, Tyson e etc., pelos momentos divertidos e descontraídos em reuniões do grupo.

Ao meu mestre de Kung Fu e amigo César, sua seposa Selma e a todos os amigos da academia Núcleo 7 Esferas do Tao por serem como uma segunda família para mim. Aos amigos de Mogi Guaçu e região, que fazem parte da minha vida, me fazendo ser a pessoa que sou hoje.

E por fim, a todos que direta ou indiretamente contribuíram para a realização deste trabalho. 


\section{Resumo}

Este trabalho tem por finalidade desenvolver um método baseado em Otimização Topológica para projetar uma estrutura mecânica, chamada de sonotrodo, acoplada a um transdutor piezelétrico de potência (dispositivo mecânico capaz de transformar energia elétrica em deformação mecânica ou vice-versa). Um sonotrodo é uma estrutura utilizada para transmitir vibrações mecânicas de um transdutor piezelétrico, ajustando a amplitude e a distribuição dos deslocamentos gerados por essa vibração para obedecer aos requisitos do projeto do transdutor. Dentre as aplicações de transdutores piezelétricos utilizando sonotrodos, pode-se citar sonares para navegação, limpeza e solda ultrassônica, tomografia acústica, furadeiras ultrassônicas, corte ultrassônico de tecidos, etc. Os requisitos de projeto do sonotrodo variam para cada aplicação, desde a necessidade de se obter o deslocamento máximo em um único ponto do sonotrodo até a uniformização do deslocamento de um plano inteiro da estrutura. Para a obtenção do resultado ótimo, neste trabalho são aplicadas técnicas de Otimização Topológica (OT). OT é um procedimento para projetar o leiaute ótimo de estruturas distribuindo material dentro de uma região fixa. O método de OT é implementado utilizando a Programação Linear Seqüencial (PLS) como algoritimo de otimização, e é baseado na interpolação "Simple Isotropic Material with Penalization" (SIMP) como formulação de modelo de material. É apresentado também um estudo sobre a utilização do modelo de material "Rational Approximation of Material Properties" (RAMP) na tentativa de se reduzir instabilidades numéricas como modos localizados. O Método de Elementos Finitos (MEF) é aplicado para modelar o sonotrodo utilizando a formulação de elemento axissimétrico de quatro nós e de elemento em Estado Plano de Tensões Mecânicas (EPTM). É apresentada a implementação para OT estrutural contínua baseada em variáveis de projeto por nós, o que minimiza instabilidades numéricas, tais como "instabilidade de tabuleiro". As freqüências de ressonâncias e os modos de vibrar são computados através do método de Lanczos. É utilizado um algoritmo baseado na formulação "Modal Assurance Criterion" (MAC) para que um certo modo de vibrar seja encontrado, de modo que a freqüência de ressonância relacionada a esse modo seja otimizada. São apresentados exemplos para verificar a eficiência e a generalidade do método proposto, e também, um estudo sobre a influência dos parâmetros de otimização utilizadas no método. Por fim, são apresentados resultados que atendem a todos os requisitos de projeto, bem como seu pós-processamento, e análise no software comercial ANSYS ${ }^{\circledR}$.

Palavras Chave: Sonotrodos para Ultrassom, Otimização Topológica, Métodos de Elementos Finitos Piezelétrico, Método de Lanczos, SIMP, RAMP. 


\section{Abstract}

This work aims at the development of a method based on Topology Optimization to design a mechanical structure, called sonotrode, which is a device usually connected to a high power piezoelectric transducer (mechanical device capable of converting electric energy into mechanical displacement or vice-versa). A sonotrode transmits mechanical vibrations of a piezoelectric transducer, adjusting the amplitude and the distribution of these vibrations to fit the design needs of the transducer. Among applications of piezoelectric transducers using sonotrodes, we can cite navigation sonars, ultrasonic cleaning and melting, acoustic tomography, ultrasonic drilling, ultrasonic fabric cutting, etc. The design needs of the sonotrode differs for each application, ranging from obtaining maximum displacement in one single point of its structure, to obtaining uniform displacements on a whole face of the sonotrode. To improve the attainment of the optimum result, in this work "Topology Optimization" (TO) is applied to design the sonotrode. TO is a procedure to design the optimal layout of structures by distributing material within a fixed domain. The objective of the developed TO formulation is to find the best topology of the sonotrode that produces maximum and uniform displacements at one of its face. The TO method is implemented using the "Sequential Linear Programming" (SLP) as the optimization algorithm, and it is based on the "Simple Isotropic Material with Penalization" (SIMP) interpolation for material model formulation. It's also presented a study about the material model "Rational Approximation of Material Properties" (RAMP), in an attempt to reduce numerical instabilities like localized modes. "Finite Element Method" (FEM) is applied to model the sonotrode considering piezoelectric four-node axisymmetric elements. A node-based design variable implementation for continuum structural topology optimization is presented to minimize numerical instabilities such as "checkerboard pattern". The ressonance frequencies and modes are computed through Lanczos Method. A "Modal Assurance Criterion" (MAC) based formulation is used to track a certain mode, so that, the ressonance frequency related to this mode can be optimized. Examples are presented to verify the efficiency and the generality of the proposed method, and also, a study about the influence of the optimization parameters used in the method. Finally, results that meets all the design requirements are presented, as well as their post-processed topology, and the analysis in the commercial software ANSYS ${ }^{\circledR}$.

Keywords: Sonotrodes for Ultrasonics, Topology Optimization, Piezoelectric Finite Element Method, Lanczos Eigensolver, SIMP, RAMP. 


\section{Lista de Figuras}

1.1 Representação esquemática de um transdutor piezelétrico de potência. . . . 20

1.2 Representação esquemática do transdutor tipo moonie. . . . . . . . . . . . 21

1.3 Representação esquemática do transdutor tipo moonie entalhado. . . . . . 22

1.4 Representação esquemática do transdutor tipo cymbal. . . . . . . . . . . 22

1.5 Representação esquemática dos sonotrodos. . . . . . . . . . . . . . . . . . 23

1.6 Representação esquemática da montagem da máquina de corte ultrassônico de tecidos. . . . . . . . . . . . . . . . . . . . . . 24

2.1 (a)Esquema de estrutura axissimétrica; (b) sistema de coordenadas; e (c) estado de tensão da estrutura. Polarização considerada na direção 2 . . . . 29

2.2 Esquema de estrutura em Estado Plano de Tensões Mecânicas (EPTM). Polarização considerada na direção 2 . . . . . . . . . . . . . . . . . . . . . . 30

2.3 Apresentação esquemática da formulação do MEF. . . . . . . . . . . . . . . 31

2.4 Mapa de coordenadas globais e locais normalizadas. . . . . . . . . . . . . . 32

2.5 Equivalência entre as coordenadas polares e cartesianas. . . . . . . . . . . . 35

2.6 Representação esquemática de um transdutor piezelétrico expandido. . . . 41

2.7 Representação de inversão de modos: antes da inversão (a) e (b), e depois

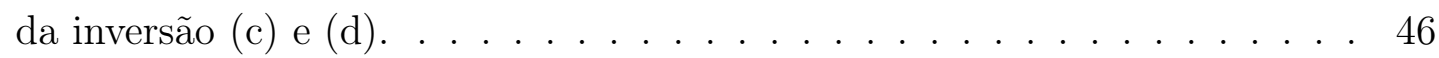

3.1 Solução de um problema por diferentes abordagens de otimização. . . . . . . 48

3.2 Procedimento típico de projeto estrutural por otimização topológica. . . . . 50

3.3 Representação de um domínio desconhecido (a) e do domínio estendido fixo(b) . . . . . . . . . . . . . . . . . . . 52

3.4 Definição do domínio de projeto. . . . . . . . . . . . . . 53

3.5 Representação dos modos localizados de uma placa em vibração. . . . . . . . 55 
3.6 Curva de interpolação da razão massa-rigidez para o modelo SIMP aplicado na densidade e no módulo de elasticidade. . . . . . . . . . . . . . . . . . 55

3.7 Curvas de interpolação da razão massa-rigidez para os modelos RAMP e SIMP

3.8 Gráficos comparativos entre a razão massa-rigidez para os vários casos de combinação entre os modelos SIMP e RAMP. . . . . . . . . . . . . . . . . 58

3.9 Representação da escala de cinza. . . . . . . . . . . . . . . . . . . . . . 59

3.10 Representação esquemática da influência da penalização $p$ no problema de OT.

3.11 Arranjo de instabilidade de xadrez. . . . . . . . . . . . . . . . . . . 60

3.12 Análise da dependência da malha.

3.13 Representação esquemática do filtro aplicado sobre os limites móveis em um problema com variáveis de projeto por elemento.

3.14 Representação de que a área de abrangência do filtro espacial não muda quando (a) é refinado para (b).

3.15 Representação esquemática do filtro em um problema com variáveis de projeto nodais.

4.1 Definição do problema de OT, do domínio de projeto e condições de contorno do problema.

4.2 Representação dos deslocamentos gerados por (a) excitação por tensão elétrica $\tilde{\phi}_{1}$ e (b) carregamento mecânico unitário $\tilde{\mathbf{t}}_{2} \ldots$. . . . . . . . . . . . 69

4.3 Representação dos deslocamentos gerados pelo carregamento $\mathbf{t}_{3}$. . . . . . . 71

5.1 Representação esquemática do filtro em um problema com variáveis de projeto nodais.

5.2 Limites móveis . . . . . . . . . . . . . . . . . . . . . . . . . 84

5.3 Diagrama de fluxo para o Método de Otimização Topológica. . . . . . . . . 85

6.1 Representação do modelo em 3D. . . . . . . . . . . . . . . . . . 86

6.2 Representação do modelo em 2D, (a) domínio completo e (b) domínio simplificado pela simetria. 
6.3 Representação da simetria utilizada no modelo 3D.

6.4 Comparação do segundo modo de vibração. Análise 2D (a) com a freqüência de ressonância em 8324,6Hz, e análise 3D, (b) vista frontal e (c) vista isométrica, com a freqüência de ressonância em 8076,6 Hz . . . . 91

6.5 Comparação do quinto modo de vibração. Análise 2D (a) com a freqüência de ressonância em $17287 \mathrm{~Hz}$, e análise 3D, (b) vista frontal e (c) vista isométrica, com a freqüência de ressonância em $17223 H z$. . . . . . . . . . 91

6.6 Comparação do sétimo modo de vibração. Análise 2D (a) com a freqüência de ressonância em $20748 \mathrm{~Hz}$, e análise 3D, (b) vista frontal e (c) vista isométrica, com a freqüência de ressonância em $20590 H z$. . . . . . . . . . 92

6.7 (a) Domínio de projeto. (b) Condiçoes de contorno. . . . . . . . . . . . . . 94

6.8 Modo de pistão da estrutura da Fig. 6.7(a). Modo alvo a ser seguido durante a otimização. . . . . . . . . . . . . . . . . . . . . . . 95

6.9 (a) Topologia ótima, (b) deformada (enfatizando o sonotrodo) da topologia ótima a $20 \mathrm{kHz}$, e (c) modo de pistão obtidos com $w_{1}=300, w_{2}=40$, $w_{3}=450, w_{4}=280, d_{0}=0,45, \Theta_{\text {lim_sup }}=55 \%, \beta=0,027 \mu \mathrm{m} \mathrm{e}$ $R_{\max }=5,5 \mathrm{~mm} \ldots \ldots \ldots \ldots \ldots \ldots$

6.10 Gráfico de convergência das funções com a vista ampliada das 50 últimas iterações. A abscissa dos gráficos representam o número de iterações e a ordenada os valores das respectivas funções. . . . . . . . . . . . . . . . . 97

6.11 Gráficos de uniformização (com vista ampliada nas últimas 50 iterações) e de freqüência. A abscissa dos gráficos representam o número de iterações e a ordenada os valores das respectivas funções. . . . . . . . . . . . . . . . 98

6.12 Topologias ótimas para $w_{2}=20, w_{3}=450, w_{4}=280, d_{0}=0,45$,

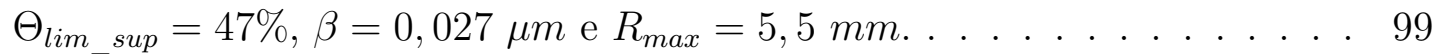

6.13 Gráficos de convergência da função $L_{1}$. . . . . . . . . . . . . . . . . . . 100

6.14 Gráficos da uniformização, razão $U_{\min } / U_{\max }$. . . . . . . . . . . . . . . . 100

6.15 Configuração dos deslocamentos na face de trabalho do sonotrodo. A abscissa representa o comprimento do sonotrodo em $m$. . . . . . . . . . 100

6.16 Topologias ótimas utilizando diferentes valores para $\beta . \quad$. . . . . . . . . . 101

6.17 Gráficos da uniformização, razão $U_{\min } / U_{\max } \ldots \ldots$. . . . . . . . . . . 102 
6.18 Topologias ótimas para $w_{1}=300, w_{3}=450, w_{4}=280, d_{0}=0,45$, $\Theta_{\text {lim_sup }}=47 \%, \beta=0,027 \mu m$ e $R_{\text {max }}=5,5 \mathrm{~mm} \ldots \ldots \ldots 2$

6.19 Gráficos de convergência da função $L_{2}$. . . . . . . . . . . . . . . . . . . . . 103

6.20 Gráficos da uniformização, razão $U_{\min } / U_{\max } \ldots$. . . . . . . . . . . . . . . 103

6.21 Configuração dos deslocamentos na face de trabalho do sonotrodo. A abscissa representa o comprimento do sonotrodo em $m$. . . . . . . . . . 103

6.22 Topologias ótimas para $w_{1}=300, w_{2}=50, w_{4}=280, d_{0}=0,45$, $\Theta_{\text {lim_sup }}=47 \%, \beta=0,027 \mu m$ e $R_{\max }=5,5 \mathrm{~mm} \ldots \ldots \ldots 4$

6.23 Configuração deformada apenas dos sonotrodos das topologias ótimas mostradas na Fig. 6.22, excitadas a 20 kHz. . . . . . . . . . . . . . . . 105

6.24 Configuração dos deslocamentos na face de trabalho do sonotrodo. A abscissa representa o comprimento do sonotrodo em $m$. . . . . . . . . . . 105

6.25 Gráficos de convergência da função $L_{3}$. . . . . . . . . . . . . . . . . . 106

6.26 Topologias ótimas para $w_{1}=300, w_{2}=50, w_{3}=450, d_{0}=0,45$, $\Theta_{\text {lim_sup }}=47 \%, \beta=0,027 \mu m$ e $R_{\max }=5,5 \mathrm{~mm} \ldots \ldots \ldots 6$

6.27 Modo alvo e modo de pistão das topologias ótimas obtidas na Fig. 6.26. . . 107

6.28 Gráficos de convergência da função $L_{4}$. . . . . . . . . . . . . . . . . . . . 108

6.29 Gráficos de convergência da freqüência do modo de pistão. . . . . . . . . . 108

6.30 Topologias ótimas utilizando diferentes valores para $d_{0} \ldots \ldots$. . . . . . 109

6.31 Gráficos da uniformização, razão $U_{\min } / U_{\max } \ldots \ldots$. . . . . . . . . . . 109

6.32 Topologias ótimas utilizando diferentes valores para $R_{\max }$. . . . . . . . 110

6.33 Gráficos da uniformização, razão $U_{\min } / U_{\max }$. . . . . . . . . . . . . . . . 110

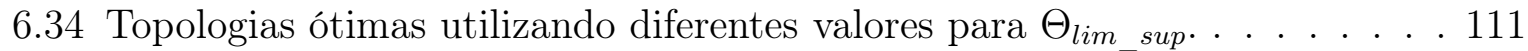

6.35 Gráficos da uniformização, razão $U_{\min } / U_{\max } \ldots \ldots$. . . . . . . . . . . 112

6.36 Topologias ótimas utilizando diferentes discretizações, com $w_{1}=300, w_{2}=$ $40, w_{3}=450, w_{4}=280, d_{0}=0,45, \Theta_{\text {lim sup }}=55 \%, \beta=0,027 \mu \mathrm{m} \mathrm{e}$

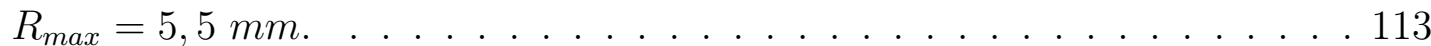

6.37 Gráficos de convergência da (a) uniformização, e (b) freqüência do modo de pistão do exemplo 1. . . . . . . . . . . . . . . . . . . . . . . . . . . 114 
6.38 (a) Topologia, (b) deformada e (c) modo de pistão do pós-processamento somente do sonotrodo da topologia apresentada no exemplo 1, omitindo o transdutor.

6.39 (a) Topologia, (b) deformada, (c) modo de pistão e (d) configuração dos deslocamentos do pós-processamento, em ANSYS ${ }^{\circledR}$, somente da topologia do sonotrodo do exemplo 1, omitindo o transdutor. . . . . . . . . . . . 116

6.40 Topologia ótima do exemplo 2 , com $w_{1}=300, w_{2}=40, w_{3}=430, w_{4}=$ $280, d_{0}=0,45, \Theta_{\text {lim_sup }}=55 \%, \beta=0,027 \mu \mathrm{m}$ e $R_{\max }=5,5 \mathrm{~mm} . \ldots 117$

6.41 Gráficos de convergência da (a) uniformização, e (b) freqüência do modo de pistão do exemplo $2 \ldots$. . . . . . . . . . . . . . . . . . 117

6.42 (a) Topologia, (b) deformada e (c) modo de pistão do pós-processamento somente da topologia do sonotrodo do exemplo 2, omitindo o transdutor. . 118

6.43 (a) Topologia, (b) deformada, (c) modo de pistão e (d) configuração dos deslocamentos do pós-processamento, obtido no ANSYS ${ }^{\circledR}$, somente da topologia do sonotrodo do exemplo 2, omitindo o transdutor. . . . . . . . . 119

6.44 Gráfico de impedância do exemplo 2. . . . . . . . . . . . . . . . . . . . . 120

6.45 Modelo simétrico 3D da estrutura com o sonotrodo otimizado. . . . . . . . 120

6.46 (a) Deformada, (b) modo de pistão, (c) configuração dos deslocamentos e (d) gráfico da impedância do modelo 3D, $\left(\right.$ ANSYS $\left.^{\circledR}\right)$. . . . . . . . . . . . . . 122

A.1 Domínio de projeto. . . . . . . . . . . . . . . . . 128

A.2 (a) Topologia ótima, (b) deformada somente do sonotrodo da topologia ótima a $3 k H z$, e (c) modo de pistão a $4,5 k H z \ldots . . . . . .128$

A.3 Gráfico de convergência das funções. A abscissa dos gráficos representam o número de iterações e a ordenada os valores das respectivas funções. . . . 129

A.4 Gráficos de uniformização e de freqüência. A abscissa dos gráficos representam o número de iterações e a ordenada os valores das respectivas funções.

A.5 Gráfico da Impedância próximo à freqüência de $3 k H z . . . . . .131$

A.6 (a) Topologia ótima, (b) deformada somente do sonotrodo da topologia ótima a $10 \mathrm{kHz}$, e (c) modo de pistão a $9,8 \mathrm{kHz}$. . . . . . . . . . . . . . 132 
A.7 Gráfico de convergência das funções. A abscissa dos gráficos representam o número de iterações e a ordenada os valores das respectivas funções. . . . 133

A.8 Gráficos de uniformização e de freqüência. A abscissa dos gráficos representam o número de iterações e a ordenada os valores das respectivas funções. . . . . . . . . . . . . . . . . . . . . . . . . . . . . . . 134

A.9 Gráfico da Impedância próximo à freqüência de $10 \mathrm{kHz}$. . . . . . . . . . . 134

B.1 (a) Modelo 3D completo, (b) simétrico e (c) 2D simétrico. . . . . . . . . 135

B.2 Deformadas da estrutura excitado em $20 \mathrm{kHz}$ do modelo 3D em (a) Vista isométrica, (b) frontal e (c) do modelo 2D simétrico. . . . . . . . . . . . 136

B.3 Gráficos das amplitudes de deslocamento dos modelos 3D e 2D, (a) no acoplamento entre amplificador e sonotrodo e, (b) na superfície superior do sonotrodo. . . . . . . . . . . . . . . . . . . . 137

B.4 Terceiro e quarto modos do modelo 3D completo, correspondentes aos modos dos modelos 3D simétrico e 2D simétrico. . . . . . . . . . . . . . 139

B.5 Exemplos de modos não-simétricos e modos em outros planos da estrutura. 140

A.1 Estrutura do PZT antes (a) e depois (b) do processo de polarização. . . . . 148

A.2 Dipolos elétricos no domínio de Weiss: (a) despolarizado; (b) durante polarização; (c) após polarização. . . . . . . . . . . . . . . . . . . . . . . . 149

A.3 Sistema de coordenadas hexagonais. . . . . . . . . . . . . . . . . 150

D.1 Extensão do teorema da reciprocidade para o meio piezelétrico. . . . . . . 158 


\section{Lista de Tabelas}

6.1 Propriedades do material PZT-8. . . . . . . . . . . . . . . . 88

6.2 Propriedades do Aço 4340 e do Alumínio 7075. . . . . . . . . . . . . . . . . 88

6.3 Teste de convergência. . . . . . . . . . . . . . . . . . . . . . . . 89

6.4 Dez primeiras freqüências de ressonância para as análises em Bidimensional (2D) e Tridimensional (3D). . . . . . . . . . . . . . . . . . . . 92

6.5 Taxa de uniformização da superfície de trabalho para algumas freqüências entre 19,5 e 20,5 kHz . . . . . . . . . . . . . . . . . . . . 121

B.1 Análise modal dos modelos 3D completo, 3D simétrico e 2D simétrico entre 18000 e $22000 \mathrm{~Hz}$. . . . . . . . . . . . . . . . . . . . . . . . 138 


\section{Lista de Abreviaturas}

ACDM Aproximação Contínua da Distribuição de Material

CAMD "Continuous Approximation of Material Distribution"

MEF Método de Elementos Finitos

MEMS "Micro-Electro-Mechanical Systems"

MOT Método de Otimização Topológica

OT Otimização Topológica

PLS Programação Linear Seqüencial

CO Critério de Optimalidade

PQS Programação Quadrática Sequencial

SIMP "Simple Isotropic Material with Penalization"

RAMP "Rational Approximation of Material Properties"

PZT Titanato Zircanato de Chumbo

EPTM Estado Plano de Tensões Mecânicas

MMA Método de movimento assintótico

TO "Topology Optimization"

SLP "Sequential Linear Programming"

FEM "Finite Element Method"

MAC "Modal Assurance Criterion"

3D Tridimensional

2D Bidimensional

IRAM "Implicity Restarted Arnoldi Method" 


\section{Conteúdo}

1 INTRODUÇÃO $\quad 19$

1.1 Transdutores Piezelétricos . . . . . . . . . . . . . . . . . . . . . 19

1.2 Histórico dos Transdutores Piezelétricos . . . . . . . . . . . . . . . . . 20

1.3 Sonotrodos para Transdutores Piezelétricos . . . . . . . . . . . . . . . . 22

1.4 Motivação . . . . . . . . . . . . . . . . . . . . . . . . . . . 24

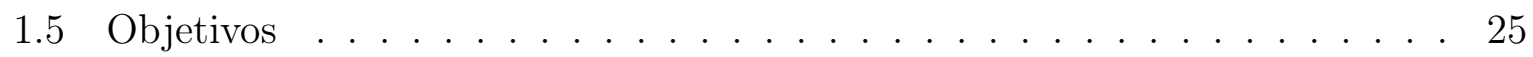

1.6 Atividades em Destaque . . . . . . . . . . . . . . . 26

2 MEF PIEZELÉTRICO 28

2.1 Formulação Axissimétrica . . . . . . . . . . . . . . . . . . . . . . . . . . . 28

2.2 Estado Plano de Tensões Mecânicas (EPTM) . . . . . . . . . . . . . . . . . 29

2.3 Implementação do Método de Elementos Finitos . . . . . . . . . . . . . . . 30

2.3.1 Formulação do Elemento Isoparamétrico de Quatro Nós . . . . . . . 31

2.4 Formulação Matricial do Elemento . . . . . . . . . . . . . . 37

2.5 Cálculo de Autovalores e Autovetores . . . . . . . . . . . . . . . . . . 40

2.6 Busca do Modo de Vibrar Baseado no Método MAC . . . . . . . . . . . . 45

3 MÉTODO DE OTIMIZAÇÃO TOPOLÓGICA (MOT) 47

3.1 Introdução . . . . . . . . . . . . . . . . . . . . . 47

3.2 Histórico . . . . . . . . . . . . . . . . . . . . . . . . . . . . . . . . . . 49

3.3 Procedimento para Implementação da Otimização Topológica . . . . . . . 50

3.4 Conceitos Teóricos da Otimização Topológica . . . . . . . . . . . . . . . 51

3.4.1 Domínio Fixo Estendido . . . . . . . . . . . . . . . . 52 
3.4 .2 Modelo de Material . . . . . . . . . . . . . . . . . . . 53

3.5 Método das Densidades . . . . . . . . . . . . . . . . . . . 53

3.6 Aspectos Numéricos da OT . . . . . . . . . . . . . . . . 58

3.6 .1 Escala de Cinza . . . . . . . . . . . . . . . . . . . 59

3.7 Instabilidade de Tabuleiro . . . . . . . . . . . . . . . . . 60

3.8 Dependência de Malha . . . . . . . . . . . . . . . . . . . . . . . . . . . 62

\section{FORMULAÇÃO DO PROBLEMA DE OT PARA O PROJETO DO SONOTRODO}

4.1 Formulação do Problema para Uniformizar os Deslocamentos . . . . . . . . 68

4.2 Formulação do Problema para Maximizar os Deslocamentos . . . . . . . . 69

4.3 Formulação do Problema para Maximizar a Rigidez da Estrutura . . . . . 70

4.4 Formulação do Problema para Atender a Função dos Autovalores .... 71

4.5 Função Multi-Objetivo . . . . . . . . . . . . . . . . . . . . . 73

4.6 Formulação Discreta das Funções Objetivo . . . . . . . . . . . . . . . . . . 74

4.7 Problema de OT na Forma Discreta . . . . . . . . . . . . . . . 75

4.8 Cálculo de Sensibilidades . . . . . . . . . . . . . . . . . . . . . . 76

5 IMPLEMENTAÇÃO NUMÉRICA 80

5.1 Modelo de Material . . . . . . . . . . . . . . . . . . . . . . 80

5.2 Implementação Numérica do MEF . . . . . . . . . . . . . . . . . . . . . . . 81

5.3 Programação Linear Seqüencial . . . . . . . . . . . . . . . . . . . . . . . 82

6 RESULTADOS $\quad 86$

6.1 Materiais utilizados . . . . . . . . . . . . . . . . 87

6.2 Teste de Convergência . . . . . . . . . . . . . . . . . . . . 88

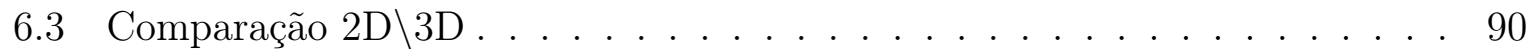

6.4 Resultados Numéricos Utilizando o Programa Implementado . . . . . . . . 93 
6.4.1 Verificação da Formulação e do Programa Implementado . . . . . . 95

6.4.2 Influência do Coeficiente $w_{1}$ e do Parâmetro de Uniformização $\beta$. . 98

6.4.3 Influência do coeficiente $w_{2} \ldots \ldots$. . . . . . . . . . . . . . . . 101

6.4.4 Influência do coeficiente $w_{3} \ldots$. . . . . . . . . . . . . . . . . . . 104

6.4.5 Influência do coeficiente $w_{4} \ldots \ldots$. . . . . . . . . . . . . 106

6.4.6 Influência do Valor Inicial das Variáveis de Projeto $\left(d_{0}\right) \ldots$. . . . . 108

6.4.7 Influência do Raio do Filtro $\left(R_{\max }\right) \ldots \ldots$. . . . . . . . . . 109

6.4.8 Influência da Restrição de Volume Superior $\left(\Theta_{\text {lim_sup }}\right)$. . . . . . . . 111

6.4.9 Conclusão sobre as Influências dos Parâmetros . . . . . . . . . . . . 112

6.5 Resultados Numéricos Utilizando Domínio mais Discretizado . . . . . . . . 112

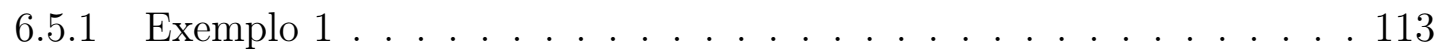

6.5 .2 Exemplo $2 \ldots \ldots \ldots \ldots$

7 CONCLUSÕES

7.1 Proposta de Trabalhos Futuros . . . . . . . . . . . . . . . . 124

Anexo A - Verificação da Generalidade da Formulação Proposta quanto à $\begin{array}{lr}\text { Uniformização dos Deslocamentos } & 127\end{array}$

A.1 Freqüência de Excitação igual a $3 k H z$. . . . . . . . . . . . . . . . . 127

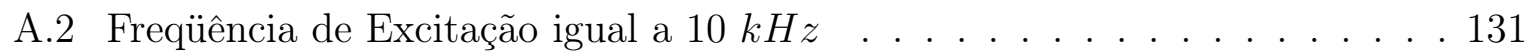

Anexo B - Comparação 2D \3D

B.1 Acoplamento Amplificador/Sonotrodo . . . . . . . . . . . . . . . . . 136

B.2 Análise modal do modelo 3D completo . . . . . . . . . . . . . . . 138

Referências

A.1 Material Piezelétrico . . . . . . . . . . . . . . . . . . . 148 
A.2 Equações Constitutivas Piezelétricas . . . . . . . . . . . . . . . . . . . 149

Apêndice B - Princípio Variacional Piezelétrico 152

Apêndice C - Implementação do Controle Elétrico por Potencial Elétrico 155

C.1 Implementação no MEF . . . . . . . . . . . . . . . . . . . . 155

C.2 Análise de Sensibilidades . . . . . . . . . . . . . . . . . . . 156

Apêndice D - Formulação da Transdução Média 158 


\section{INTRODUÇÃO}

Neste capítulo são discutidos alguns conceitos básicos, aplicações e histórico de transdutores piezelétricos, conceitos e aplicações de sonotrodos, a motivação de se utilizar OT no projeto de sonotrodos e os objetivos deste trabalho.

\subsection{Transdutores Piezelétricos}

Os transdutores piezelétricos de potência são encontrados em projetos como sonares para navegação (já utilizados há mais de 50 anos) (DESILETS et al., 1999), projetos de ultra-som de alta potência, transmissão acústica de dados em baixo da água, limpeza e solda ultrassônica (SHUYU, 1995, 2004, 2005, 1997), em projetos como sonar para tomografia acústica e monitoramento do oceano global (MOROZOV; WEBB, 2003), processos químicos (HEIKKOLA; LAITINEN, 2005), máquinas de corte e solda ultrassônica de tecidos (LUCAS; GRAHAM; SMITH, 1996), e até no projeto de furadeiras ultrassônicas (SHERRIT et al., 2000), recentemente pesquisado pela NASA para expedições para Marte ou outros planetas com baixa gravidade.

Os transdutores piezelétricos de potência se baseiam no efeito piezelétrico, que é definido como a conversão de energia mecânica em elétrica (efeito direto) ou a conversão de energia elétrica em mecânica (efeito inverso), ou seja, é um fenômeno físico pelo qual aparece uma tensão elétrica entre as faces de um material quando este é submetido a uma pressão mecânica. O efeito inverso também se aplica, surgindo uma deformação mecânica ao ser aplicada uma tensão elétrica. Os transdutores piezelétricos de potência (Fig. 1.1) são construídos, em geral, na forma de sanduíche, ou seja, as cerâmicas piezelétricas são comprimidas entre duas massas metálicas por um parafuso de alta resistência mecânica. As cerâmicas são polarizadas na direção longitudinal e são montadas com os sentidos de polarização alternados a cada cerâmica, possibilitando a ligação em paralelo. As duas massas de metal diminuem a freqüência de ressonância do transdutor bem abaixo do que se fosse apenas uma pilha de piezocerâmicas (DESILETS et al., 1999) devido à adição de 
massa ao sistema. O pré-tensionamento das cerâmicas piezelétricas evita a fratura destas devido a altas tensões aplicada às cerâmicas na ressonância (ARNOLD; MUHLEN, 2001b). O valor típico para essa pré-tensão é de 30 MPa (ARNOLD; MUHLEN, 2001a).

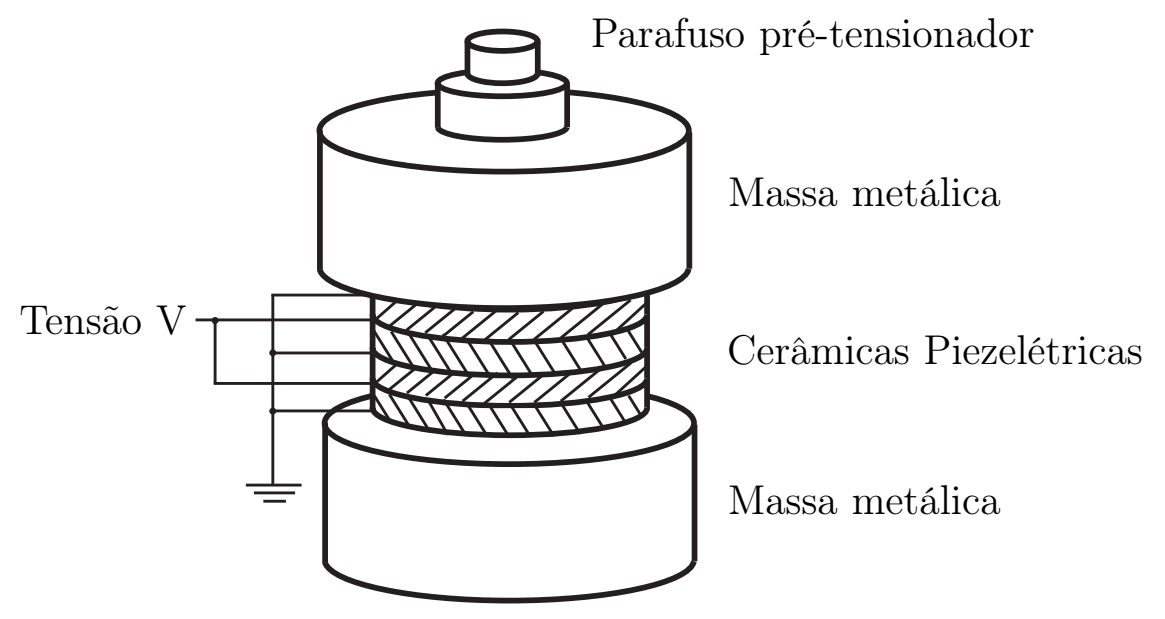

Figura 1.1: Representação esquemática de um transdutor piezelétrico de potência.

Esse tipo de montagem, utilizando massas metálicas, permite que o transdutor seja projetado para que a sua primeira freqüência de ressonância do modo longitudinal esteja na faixa entre $20 k H z$ e $40 k H z$ (SILVA, 2006), utilizando-se cerâmicas piezelétricas com espessura de apenas $5 \mathrm{~mm}$. Essa restrição deve ser imposta no projeto pois é a menor faixa de freqüência ultrassônica que se pode trabalhar, pois não engloba a freqüência audível pelo ser humano, que está na faixa de $20 \mathrm{~Hz}$ até $20000 \mathrm{~Hz}$. Se, por exemplo, um equipamento ultrassônico de aproximadamente $1 \mathrm{~kg}$ vibrar a uma freqüência de $18 \mathrm{kHz}$, o ruido seria insuportável ao ouvido humano, sendo necessário um sistema de isolamento acústico, o que aumentaria significantivamente o custo do projeto.

\subsection{Histórico dos Transdutores Piezelétricos}

O projeto dos primeiros atuadores piezelétricos era baseado em modelos analíticos simples (XU et al., 1991; DOGAN; UCHINO; NEWNHAM, 1997) e técnicas experimentais (DOGAN; UCHINO; NEWNHAM, 1997). Inicialmente, a otimização dos atuadores piezelétricos limitava-se a dimensões específicas (por exemplo, a espessura da piezocerâmica). Os modelos analíticos eram unidimensionais e aplicados somente a geometrias muito simples, e portanto, deficientes quando aplicados a transdutores com geometrias complexas (BRISSAUD, 1991).

O atuador piezelétrico do tipo moonie (Fig. 1.2), um exemplo clássico de atuador piezelétrico definido como um sanduíche de um disco piezocerâmico entre capas de metal, 
onde cada uma das capas tem a forma de meia lua na cavidade interna da superfície (por isso o nome de moonie), utilizado como transformador mecânico para converter e amplificar o deslocamento lateral da piezocerâmica em deslocamento axial maior na estrutura flexível (XU et al., 1991). Os atuadores moonie são utilizados na geração de ondas ultrassônicas, por exemplo, em hidrofones (XU et al., 1991; ONITSUKA et al., 1995). Os atuadores moonie foram caracterizados através de técnicas experimentais e modelos analíticos considerando como parâmetros os deslocamentos, a força gerada e a freqüência de ressonância (XU et al., 1991; DOGAN; UCHINO; NEWNHAM, 1997).

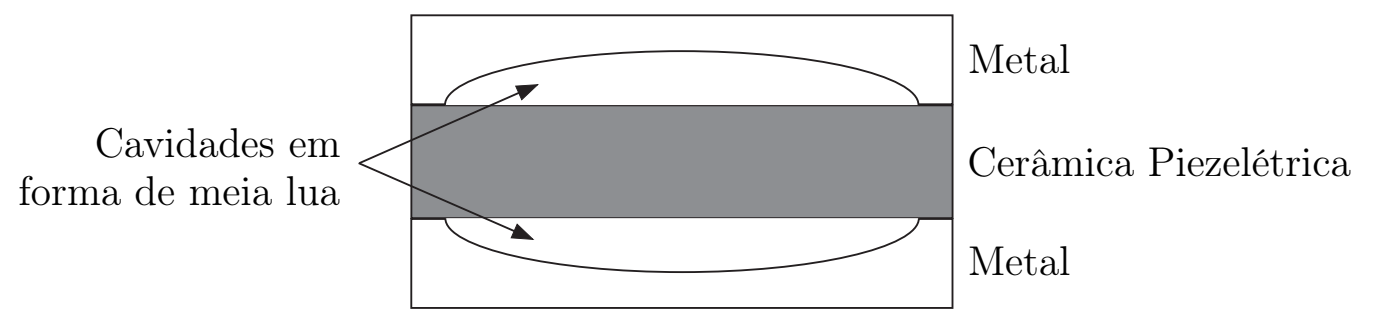

Figura 1.2: Representação esquemática do transdutor tipo moonie.

O avanço no desenvolvimento dos atuadores piezelétricos somente foi possível com a teoria e elementos finitos piezelétrica desenvolvida por Allik e Hughes (1970), possibilitando modelar e otimizar o desempenho dos atuadores piezelétricos considerando geometrias complexas, obtendo novas configurações. Assim, a construção dos protótipos passou a ser necessária apenas para verificações dos resultados, como no caso dos sonares (NAILLON; COURSANT; BESNIER, 1983).

A análise de estruturas piezelétricas foi implementada pela primeira vez num software comercial de MEF por Ostergaard e Pawlak (1986). Assim, as simulações utilizando o MEF piezelétrico passaram a ser utilizadas naturalmente para otimizar dispositivos piezelétricos, tais como, transdutores ultrassônicos para formação de imagens médicas (LERCH, 1990). Conseqüentemente, os atuadores moonie foram otimizados, considerando a freqüência de ressonância, o deslocamento e a força de blocagem como parâmetros e otimização (NEWNHAM et al., 1993; ONITSUKA et al., 1995). Portanto, o MEF piezelétrico possibilitou substituir o método de otimização baseado na intuição do projetista por um método de otimização sistemático, ou seja, utilizando a otimização paramétrica (CHALLANDE, 1990). Assim, o transdutor denominado moonie entalhado (ou "groove moonie") (Fig. 1.3) foi obtido a partir de um estudo da flexibilidade do transdutor moonie, verificando a possibilidade de remover material da estrutura acoplada, gerando entalhes na estrutura. O entalhe aumenta a flexibilidade da parte exterior do acoplamento, provendo um deslocamento gerado maior do que o atuador moonie. 


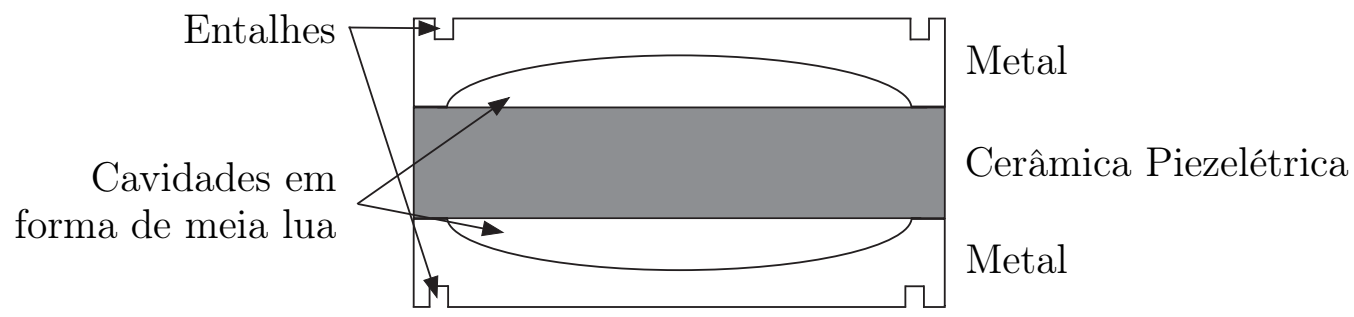

Figura 1.3: Representação esquemática do transdutor tipo moonie entalhado.

Neste caminho, uma variação do atuador moonie entalhado deu origem ao atuador cymbal (DOGAN; UCHINO; NEWNHAM, 1997) (Fig. 1.4). No atuador cymbal o deslocamento gerado é obtido pela combinação de movimentos de flexão e rotação. No entanto, os atuadores moonie apresentam somente movimentos de flexão. Portanto, a evolução dos atuadores moonie proporcionou obter deslocamentos e força de blocagem maiores devido ao aumento da rigidez axial. Analisando o desempenho dos atuadores piezelétricos, concluiu-se que grandes deslocamentos e uma alta força de blocagem são soluções de compromisso, e dependem da rigidez e da flexibilidade da estrutura acoplada a piezocerâmica.

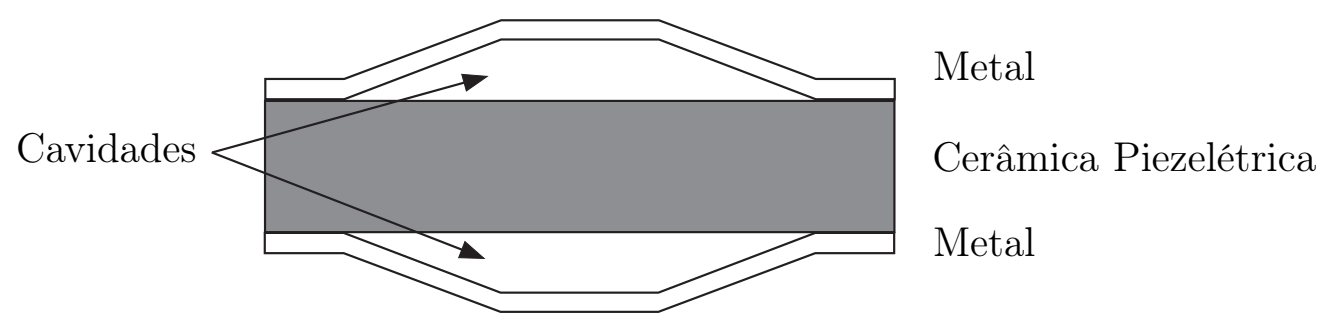

Figura 1.4: Representação esquemática do transdutor tipo cymbal.

\subsection{Sonotrodos para Transdutores Piezelétricos}

Existe uma grande variedade de aplicações para os transdutores piezelétricos de potência, as quais, na maioria dos projetos, inclui um sonotrodo acoplado ao transdutor.

Sonotrodos são elementos utilizados em várias aplicações de potência onde eles geralmente operam como uma ferramenta agindo diretamente na superfície de trabalho (CARDONI; LUCAS, 2002), ou transimindo energia para outros dispositivos. A forma geométrica do sonotrodo varia de acordo com a aplicação, dependendo do padrão de deslocamento a ser produzido, podendo ser cilíndrico (PARRINI, 2001, 2003), na forma de um bloco (ENSMINGER, 1988; LUCAS; GRAHAM; SMITH, 1996; GRAHAM; PETZING; LUCAS, 1999; CARDONI; LUCAS, 2002; CARDONI et al., 2004; ADACHI; UEHA, 1990), ou do tipo lâmina-larga (ENSMINGER, 1988). Tanto os sonotrodos cilíndricos quanto os do 


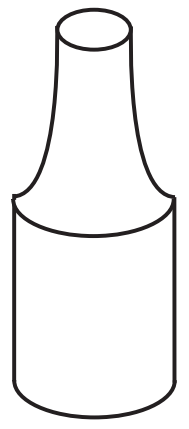

(a) Cilíndrico

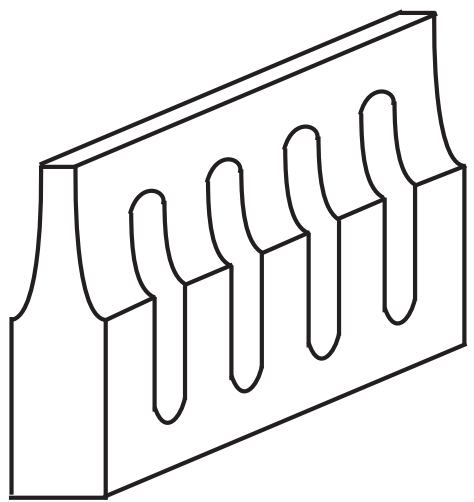

(b) Lâmina Larga

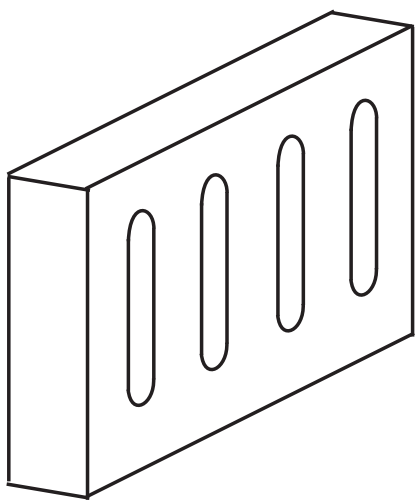

(c) Bloco

Figura 1.5: Representação esquemática dos sonotrodos.

tipo lâmina-larga e bloco são projetados para vibrar em modo longitudinal, sendo que a amplitude de vibração na parte superior e inferior é máxima, e na região central é mínima. Os sonotrodos cilíndrico e de lâmina-larga possuem geralmente um perfil escalonado, o qual produz uma relação de amplificação que é dada pela relação entre as áreas nas duas pontas do sonotrodo.

Em sonotrodos do tipo lâmina-larga e bloco, utilizam-se fendas passantes (CARDONI; LUCAS, 2002; ADACHI; UEHA, 1990) paralelas à direção de movimento longitudinal do sonotrodo. Elas têm como objetivo uniformizar a distribuição de vibração ao longo da face de trabalho do sonotrodo quando o sistema se encontra em ressonância. Um sonotrodo produzido sem essas fendas teria um deslocamento muito elevado nas extremidades da face de trabalho, e ao mesmo tempo, um deslocamento muito pequeno na sua região central (SILVA, 2006). A utilização de fendas visa melhorar a distribuição de vibração ao longo da face de trabalho do sonotrodo, pois, para garantir a qualidade do processo, é necessário que essa distribuição de vibração seja uniforme, isto é, os deslocamentos tanto no centro quanto nas extremidades da face de trabalho devem vibrar com amplitudes próximas. A uniformização é calculada como a razão entre as amplitudes mínima e máxima $\left(U_{\min } / U_{\max }\right)$, então, para que o processo produza resultados satisfatórios, o valor da uniformização deve ser maior que 80\% (CARDONI; LUCAS, 2002).

Uma aplicação interessante para a utilização de sonotrodos é no processo de corte ultra-sônico de tecidos (LUCAS; GRAHAM; SMITH, 1996; CARDONI; LUCAS, 2002; SILVA, 2006), onde é utilizado um transdutor piezelétrico de potência para gerar os deslocamentos, um sonotrodo para amplificação mecânica de deslocamentos e o sonotrodo do tipo lâmina-larga. O esquema de montagem desse sistema pode ser visto na Fig. 1.6. 


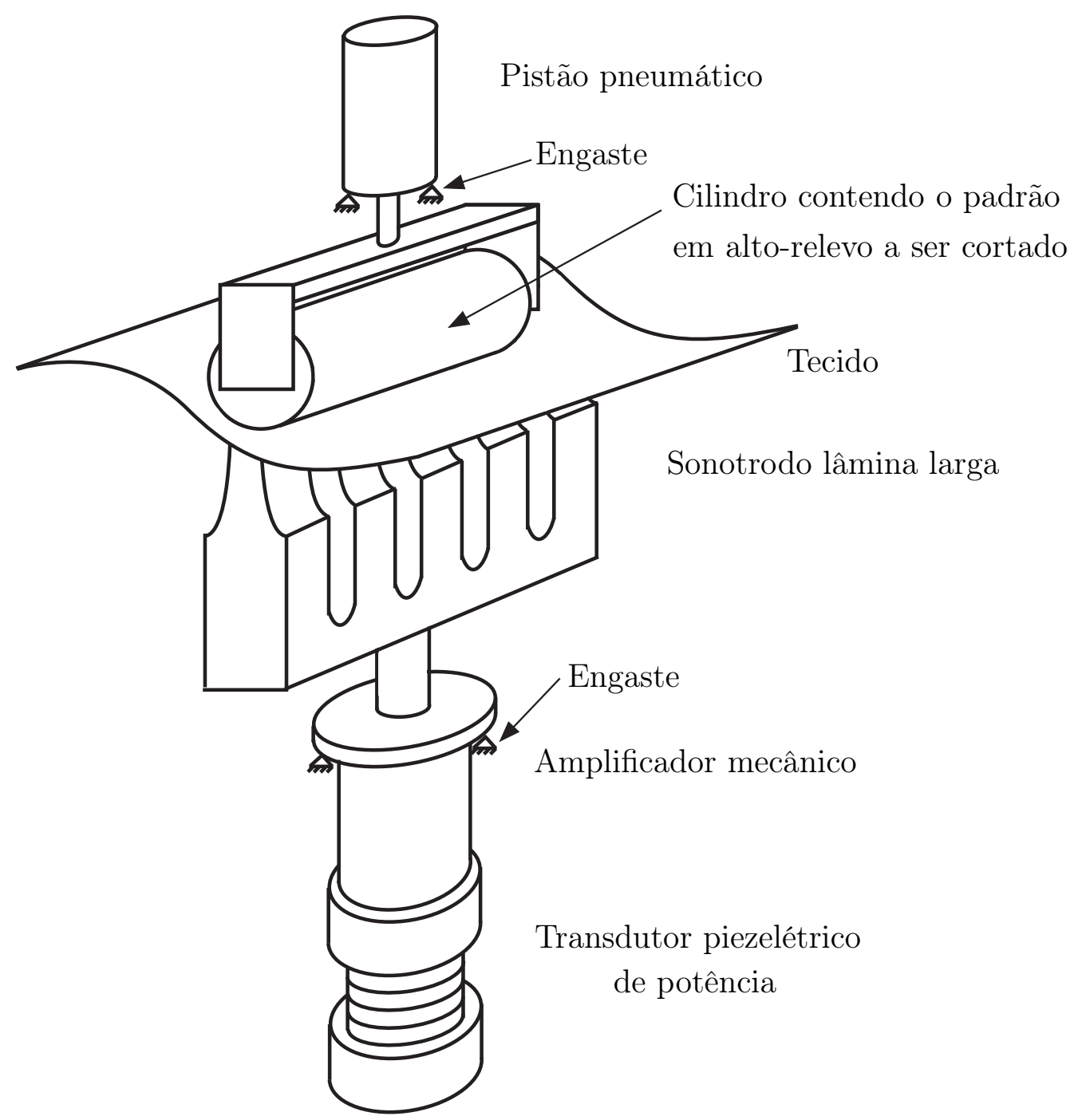

Figura 1.6: Representação esquemática da montagem da máquina de corte ultrassônico de tecidos.

\subsection{Motivação}

Como dito anteriormente, transdutores piezelétricos de potência vêm sendo amplamente utilizados no desenvolvimento de projetos como limpeza e solda ultra-sônica, sonares para navegação, tomografia acústica, furadeiras ultra-sônicas, corte ultra-sônico de tecidos, etc.

Trabalhos desenvolvidos por (ADACHI; UEHA, 1990; LUCAS; GRAHAM; SMITH, 1996; CARDONI; LUCAS, 2002; CARDONI et al., 2004), utilizaram-se de métodos não sistemáticos para a obtenção de uma topologia ótima, como o método de tentativa e erro (ADACHI; UEHA, 1990), embora para alguns casos, por exemplo no caso da máquina de corte ultrassônico de tecidos que utiliza o sonotrodo do tipo lâmina-larga, a uniformização 
que as fendas proporcionam ao sonotrodo é suficiente. A uniformização é medida como a razão entre as amplitudes mínima e máxima na superfície de trabalho, $U_{\min } / U_{\max }$, que no caso desse tipo de sonotrodo com fendas, é igual a 89, 3\% (SILVA, 2006).

A aplicação de técnicas de otimização no projeto de sonotrodo não é recente, destacando-se o projeto das "cornetas acústicas" (ou "acoustic horn"), um tipo muito utilizado de sonotrodo para transmissão, recepção e amplificação mecânica de ondas acústicas geradas pela cerâmica piezelétrica (BANGTSSON; NORELAND; BERGGREN, 2003; MOFFETT; POWERS; JEVNAGER, 1998). As configurações das cornetas anteriormente utilizadas refletiam as ondas não-planas geradas pelo próprio transdutor. A otimização aplicada nesse caso é utilizada para minimizar essa reflexão indesejada das ondas. O método utilizado nesse projeto foi a otimização de forma, a qual procura a estrutura ótima modificando os contornos da corneta.

Estudos recentes feitos por Silva (2006), revelam que, aplicando otimização paramétrica em sonotrodos cilíndricos (Fig. 1.5(a)), é possível aumentar a uniformização do perfil de deslocamentos na face de trabalho de $72,3 \%$ para $85,8 \%$ no modelo em elementos finitos, e de 77,9\% para 87,8\% nas medições feitas no protótipo.

Dessa forma, a aplicação de técnicas de otimização topológica no projeto do sonotrodo visa a obtenção da topologia ótima da estrutura de acordo com a necessidade de cada aplicação, resultando num processo mais eficiente, sistemático e com menor custo computacional que os outros métodos, garantindo que o resultado seja o melhor possível.

\subsection{Objetivos}

Neste trabalho é apresentado o desenvolvimento de um método baseado em Otimização Topológica (OT) para projetar a estrutura mecânica chamada sonotrodo, a qual é acoplada à um transdutor piezelétrico de potência. Para isso, o trabalho envolve o estudo sobre transdutores piezelétricos de potência, Método de Elementos Finitos (MEF) aplicado a materiais piezelétricos e também, um estudo mais aprofundado sobre o Método de Otimização Topológica (MOT). Foi implementado um algoritmo em MatLab, utilizando o MEF e o MOT, para otimizar a estrutura e sua freqüência de ressonância.

Dessa forma, o objetivo deste trabalho é aplicar técnicas de otimização topológica no projeto do sonotrodo visando a obtenção da topologia ótima da estrutura de modo a garantir a maximização e a uniformização da distribuição dos deslocamentos em um 
mesmo plano fazendo com que a freqüência de um certo modo de vibrar seja próxima ou maior que um dado valor de freqüência. A meta de uniformização é fazer com que a razão entre as amplitudes mínima e máxima da face de trabalho do sonotrodo $\left(U_{\min } / U_{\max }\right)$ seja maior que $80 \%$.

A aplicação de técnicas de otimização topológica torna o processo mais eficiente, sistemático e com menor custo computacional.

\subsection{Atividades em Destaque}

Nesta Dissertação de Mestrado são abordados diversos assuntos como trasdutores piezelétricos, MEF e MOT, e dentre eles, alguns merecem maior destaque, tais como:

- Formulação do Elemento Axissimétrico Piezelétrico - esta formulação é utilizada para modelar estruturas cilíndricas piezelétricas, que neste trabalho são as cerâmicas piezelétricas. Para tanto, foi necessária a completa implementação da formulação de elemento axissimétrico piezelétrico. Embora softwares comerciais de elementos finitos (como por exemplo o Ansys ${ }^{\circledR}$ ) já utilizem esse tipo de formulação, na literatura somente foram encontrados referências que descreviam a formulação de elemento axissimétrico não-piezelétrico (COOK; MALKUS; PLESHA, 1989), e a formulação de elemento plano piezelétrico (BATHE, 1995).

- Cálculo de Autovalor e Autovetor Piezelétrico - neste trabalho, deseja-se otimizar a freqüência de ressonância de um certo modo de vibrar, então o cálculo de autovalor e autovetor é necessário para encontrar a freqüência de ressonância e o modo de vibrar desejados. Porém, a rotina utilizada (função "eigs" do MatLab) calcula autovalores e autovetores de estruturas não-piezelétricas. Para isso, foi utilizada uma modificação baseada nas modificações propostas por Yong e Cho (1994), Yong (1995), Yong e Cho (1996), a qual possibilita a utilização dessa rotina para estruturas piezelétricas.

- "Modal Assurance Criterion" (MAC) - como o objetivo é otimizar a freqüência de ressonância associada a um certo modo de vibrar, é necessário, após encontrados os autovalores e autovetores, fazer a busca do modo de vibrar desejado dentre aqueles que foram calculados. Para fazer tal comparação numérica, foi utilizada a formulação do MAC (EWINS, 1986; KIM; KIM, 2000) que compara dois modos de vibrar e fornece o grau de igualdade entre esses dois modos.

- Discussão SIMP/RAMP - o modelo de material é uma equação que define a mistura 
em micro-escala de dois ou mais materiais (um deles pode ser "vazio"), permitindo passar da condição de zero material ("buraco") a sólido em cada ponto do domínio. Para isso, foram utilizados dois modelos de material, o SIMP e o RAMP. Neste trabalho, é apresentada uma discussão detalhada avaliando diferentes combinações na utilização dos modelos nas matrizes de rigidez e massa e com diferentes valores de penalizações.

- Aproximação Contínua da Distribuição de Material (ACDM) - técnica utilizada para reduzir a "instabilidade de tabuleiro" provocada por instabilidades numéricas proveniente das formulações de MEF e OT. A "instabilidade de tabuleiro" ocorre pois, numericamente, uma estrutura com elementos dispostos na forma de tabuleiro, é mais rígida que uma estrutura homogênea.

- Técnica de Projeção - técnica utilizada para eliminar a dependência da malha do problema de OT, baseada no trabalho de Guest, Prevost e Belytschko (2004). Esta técnica também auxilia na redução da "instabilidade de tabuleiro".

- Formulação para Uniformização de Deslocamentos - a formulação proposta nessa Dissertação pode ser considerada como uma contribuição científica para a área de OT aplicada a dispositivos ultrassônicos, pois até dado momento, não foi encontrada nenhuma formulação específica para tal caso, onde é necessária a uniformização das amplitudes de toda uma superfície de uma estrutura vibrando em freqüências ultrassônicas. 


\section{MEF PIEZELÉTRICO}

Um método genérico, como o Método de Elementos Finitos (MEF), é necessário para análises estruturais de estruturas com topologias genéricas. Para isso, uma formulação de MEF linear para piezeletricidade deve ser aplicada.

A teoria sobre piezeletricidade e as equações constitutivas piezelétricas que definem o comportamento de um material piezelétrico linear podem ser encontradas no Apêndice A.

São apresentadas duas formulações de elementos utilizadas nesse trabalho, a axissimétrica e a de Estado Plano de Tensões Mecânicas (EPTM).

A formulação axissimétrica consiste numa aproximação bidimensional para a simulação de estruturas com geometria axissimétrica (ou seja, estruturas rotativas) sujeitas a carregamentos axissimétricos.

A formulação de elementos EPTM também consiste em uma aproximação bidimensional, mas para estruturas planas finas e que possuem carregamento apenas no plano. Ou seja, estruturas podem ser consideradas como estruturas em EPTM desde que sua dimensão característica no plano em que estão aplicados os carregamentos seja significantemente maior do que a espessura perpendicular a este plano, que é a situação deste trabalho.

\subsection{Formulação Axissimétrica}

Essa consideração é utilizada em corpos que possuem uma geometria rotativa sujeita a carregamentos axissimétricos, apresentado na Fig. 2.1.

Neste tipo de formulação, têm-se como hipóteses (COOK; MALKUS; PLESHA, 1989):

$$
T_{13}=T_{23}=S_{13}=S_{23}=D_{3}=0
$$

Então, considerando a Eq. (2.1), a matriz de equações constitutivas piezelétricas 


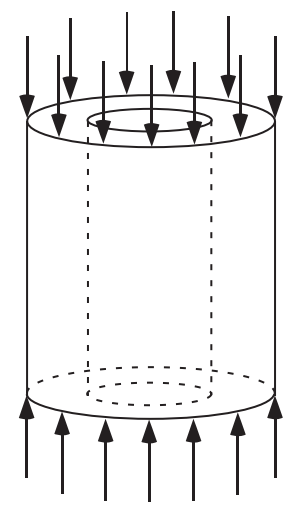

(a)

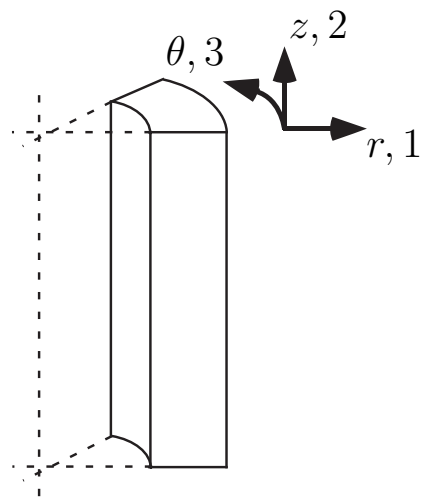

(b)

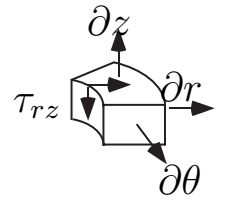

(c)

Figura 2.1: (a)Esquema de estrutura axissimétrica; (b) sistema de coordenadas; e (c) estado de tensão da estrutura. Polarização considerada na direção 2.

(Eq. (A.4)) pode ser reduzida, sendo dada por:

$$
\left\{\begin{array}{c}
T_{11} \\
T_{22} \\
T_{33} \\
T_{12} \\
D_{1} \\
D_{2}
\end{array}\right\}=\left[\begin{array}{cccccc}
c_{11}^{E} & c_{13}^{E} & c_{12}^{E} & 0 & 0 & e_{13} \\
c_{13}^{E} & c_{33}^{E} & c_{13}^{E} & 0 & 0 & e_{33} \\
c_{12}^{E} & c_{13}^{E} & c_{11}^{E} & 0 & 0 & e_{13} \\
0 & 0 & 0 & c_{44}^{E} & e_{15} & 0 \\
0 & 0 & 0 & e_{15} & -\varepsilon_{11}^{S} & 0 \\
e_{13} & e_{33} & e_{13} & 0 & 0 & -\varepsilon_{33}^{S}
\end{array}\right]\left\{\begin{array}{c}
S_{11} \\
S_{22} \\
S_{33} \\
S_{12} \\
-E_{1} \\
-E_{2}
\end{array}\right\}
$$

e a matriz de constantes elásticas para o material isotrópico (BATHE, 1995) fica:

$$
\left\{\begin{array}{l}
T_{11} \\
T_{22} \\
T_{33} \\
T_{12}
\end{array}\right\}=\frac{E}{(1+\nu)(1-2 \nu)}\left[\begin{array}{cccc}
1-\nu & \nu & \nu & 0 \\
\nu & 1-\nu & \nu & 0 \\
\nu & \nu & 1-\nu & 0 \\
0 & 0 & 0 & \frac{1-2 \nu}{2}
\end{array}\right]\left\{\begin{array}{c}
S_{11} \\
S_{22} \\
S_{33} \\
S_{12}
\end{array}\right\}
$$

onde $E$ é o módulo de elasticidade e $\nu$ o coeficiente de Poisson do material.

\subsection{Estado Plano de Tensões Mecânicas (EPTM)}

Esta consideração é utilizada em corpos que possuem uma geometria com a largura (direção 1) e o comprimento (direção 2) com dimensões comparáveis e no entanto, muito maiores que a espessura (direção 3), como mostra a Fig. 2.2. As cargas mecânicas são aplicadas uniformemente sobre a espessura da placa. 


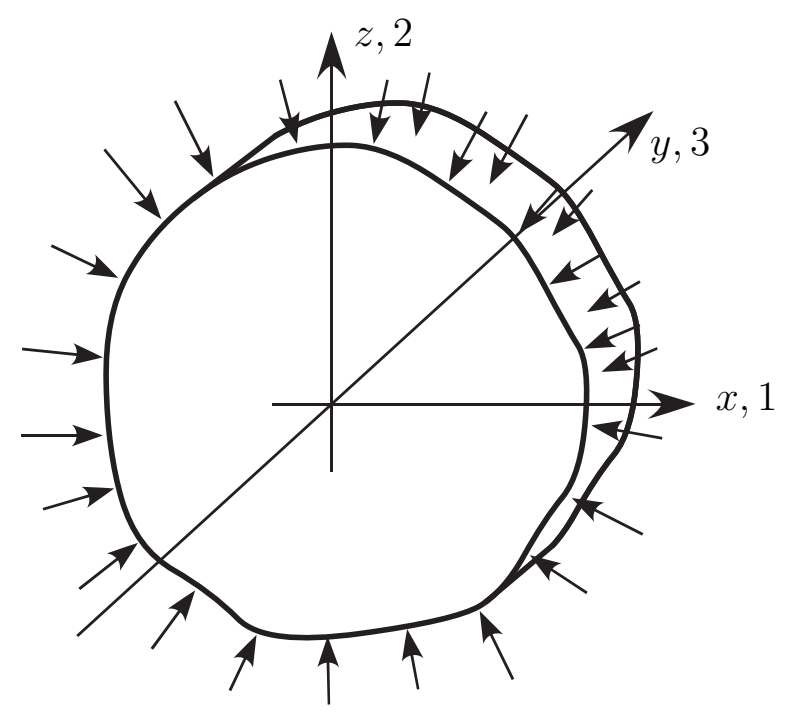

Figura 2.2: Esquema de estrutura em Estado Plano de Tensões Mecânicas (EPTM). Polarização considerada na direção 2.

No Estado Plano de Tensões Mecânicas, têm-se como hipóteses:

$$
T_{33}=T_{13}=T_{23}=D_{3}=0
$$

Assim como no elemento axissimétrico, considera-se a Eq. (2.4), reduzindo a matriz de equações constitutivas piezelétricas (Eq. (A.4)), que passa a ser dada por:

$$
\left\{\begin{array}{l}
T_{11} \\
T_{22} \\
T_{12} \\
D_{1} \\
D_{2}
\end{array}\right\}=\left[\begin{array}{ccccc}
c_{11}^{E}-\frac{c_{12}^{E}}{c_{11}^{E}} & c_{13}^{E}-\frac{c_{12}^{E} c_{13}^{E}}{c_{11}^{E}} & 0 & 0 & e_{13}-\frac{e_{13} c_{12}^{E}}{c_{11}^{E}} \\
c_{13}^{E}-\frac{c_{12}^{E} c_{13}^{E}}{c_{11}^{E}} & c_{33}^{E}-\frac{c_{13}^{E}}{c_{11}^{E}} & 0 & 0 & e_{33}-\frac{e_{13} c_{13}^{E}}{c_{11}^{E}} \\
0 & 0 & c_{66}^{E} & e_{15} & 0 \\
0 & 0 & e_{15} & -\varepsilon_{11}^{S} & 0 \\
e_{13}-\frac{e_{13} c_{12}^{E}}{c_{11}^{E}} & e_{33}-\frac{e_{13} c_{13}^{E}}{c_{11}^{E}} & 0 & 0 & -\varepsilon_{33}^{S}-\frac{e_{13}^{2}}{c_{11}^{E}}
\end{array}\right]\left\{\begin{array}{c}
S_{11} \\
S_{22} \\
S_{12} \\
-E_{1} \\
-E_{2}
\end{array}\right\}
$$

e a matriz de constantes elásticas para o material isotrópico (BATHE, 1995) fica:

$$
\left\{\begin{array}{l}
T_{11} \\
T_{22} \\
T_{12}
\end{array}\right\}=\frac{E}{(1+\nu)(1-2 \nu)}\left[\begin{array}{ccc}
1-\nu & \nu & 0 \\
\nu & 1-\nu & 0 \\
0 & 0 & \frac{1-2 \nu}{2}
\end{array}\right]\left[\begin{array}{c}
S_{11} \\
S_{22} \\
S_{12}
\end{array}\right\}
$$

\subsection{Implementação do Método de Elementos Finitos}

O MEF consiste num método de transformação de um problema, apresentado na forma integral, em um problema aproximado de álgebra linear, onde os coeficientes são integrais avaliadas sobre os elementos finitos da estrutura ou meio. Para se iniciar uma 
análise de elementos finitos como descrito na Fig. 2.3, é necessário que o modelo a ser analisado seja discretizado em uma malha de elementos finitos, que é a representação do domínio de projeto em subdomínios, cujas dimensões devem ser pequenas o suficiente para permitir a aproximação do problema (BATHE, 1995; NAILLON; COURSANT; BESNIER, 1983).

A formulação do MEF é esquematizada como na figura abaixo (Fig. 2.3).

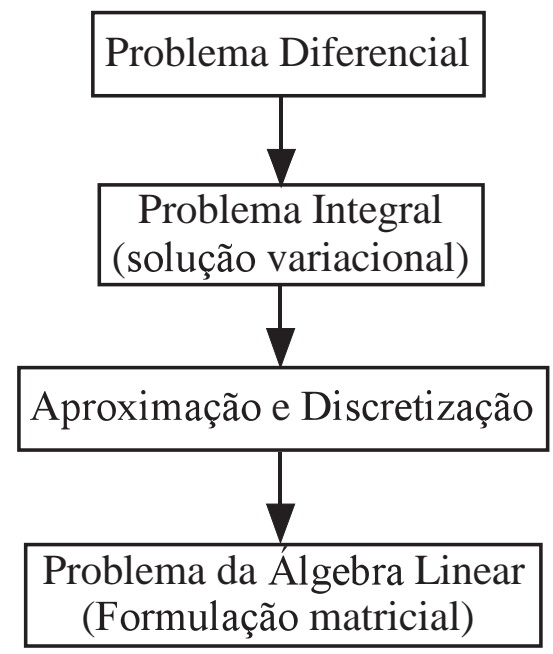

Figura 2.3: Apresentação esquemática da formulação do MEF.

Como visto no Apêndice B, o problema na forma diferencial foi transformado para a forma integral utilizando-se o princípio variacional. Em seguida é necessário discretizar o domínio de projeto para que se possa fazer a aproximação por integração numérica. Para isso, utiliza-se a formulação de elemento isoparamétrico que, neste trabalho, será o elemento de quatro nós. E por fim, utiliza-se o conceito de funções de forma da formulação de elemento isoparamétrico para obter a formulação matricial das equações de equilíbrio piezelétrico.

\subsubsection{Formulação do Elemento Isoparamétrico de Quatro Nós}

Para entender o conceito de elemento isoparamétrico, é necessário entender o conceito de coordenadas locais normalizadas.

Para construir as matrizes de rigidez e de massa globais de uma estrutura (necessárias para a modelagem do sistema), é necessário que se trabalhe no sistema de coordenadas globais, pois ele indica onde cada nó do domínio se encontra. Contudo, essas matrizes globais são construídas utilizando-se as matrizes de massa e de rigidez locais de cada elemento. O cálculo dessas matrizes locais é simplificado utilizando-se as coordenadas 
locais normalizadas, e então, uma matriz de transformação é utilizada para que as coordenadas locais das matrizes locais, se transformem em cordenadas globais, para assim poderem ser combinadas, montando as matrizes globais.

No sistema de coordenadas locais normalizadas $(\xi, \eta)$, o elemento é quadrado (dimensão 2x2) com o centro de coordenadas no centro do elemento (Fig. 2.4(a)). Já para o sistema de coordenadas globais $(x, z)$ o elemento é distorcido da forma retangular (Fig. 2.4(b)).

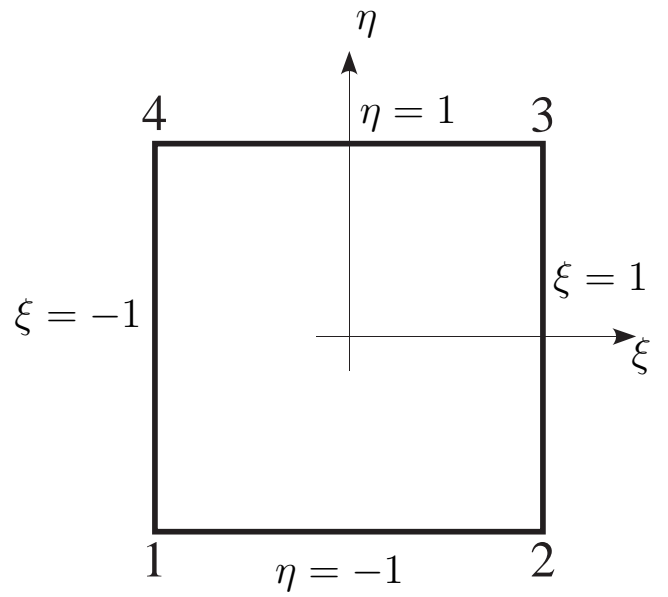

(a) coordenadas normalizadas

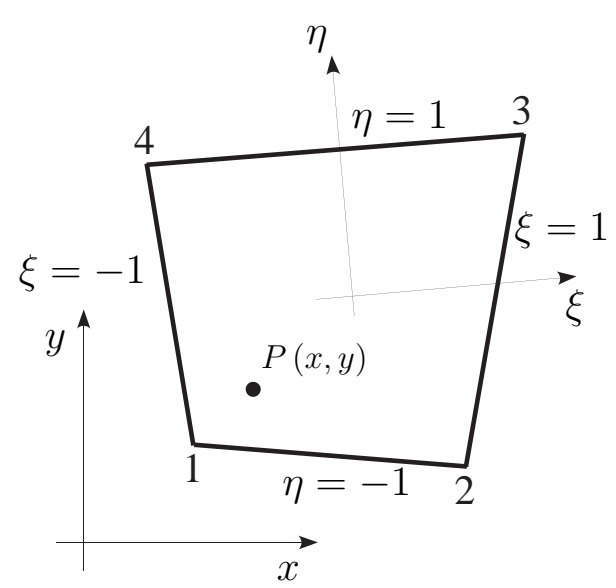

(b) coordenadas globais

Figura 2.4: Mapa de coordenadas globais e locais normalizadas.

Com isso, pode-se definir elemento isoparamétrico como aquele que, dentro do espaço de coordenadas locais normalizadas, utiliza as mesmas funções de interpolação para calcular, através de integração numérica, diferentes propriedades físicas, como deslocamento mecânico e elétrico, coordenadas dos nós, condução térmica, distribuição de tensões, etc. Essas funções de interpolação são definidas para cada nó do elemento, diferente entre si, e são chamadas de funções de forma.

As funções de forma $\mathbf{N}_{\mathbf{u}}$ são funções do sistema de coordenadas locais normalizadas, e as coordenadas de qualquer ponto $P$ podem ser expressas em termos das coordenadas globais $(x, z)$ dos nós:

$$
\left\{\begin{array}{l}
x(\xi, \eta) \\
z(\xi, \eta)
\end{array}\right\}=\left[\mathbf{N}_{\mathbf{u}}\right]\{\mathbf{x}\}_{e}
$$

onde $\{\mathbf{x}\}_{e}$ é o vetor de coordenadas nodais do elemento, ou seja:

$$
\{\mathbf{x}\}_{e}=\left\{\begin{array}{llllllll}
x_{1} & z_{1} & x_{2} & z_{2} & x_{3} & z_{3} & x_{4} & z_{4}
\end{array}\right\}^{t}
$$


Os deslocamentos mecânicos e o potencial elétrico podem ser expressos para cada elemento, através dos seus respectivos valores nodais $\left\{\mathbf{U}_{e}\right\}$ e $\left\{\phi_{e}\right\}$ pelas funções de forma $\left[\mathbf{N}_{\mathbf{u}}\right]$ e $\left[\mathbf{N}_{\phi}\right]$, ou seja:

$$
\begin{aligned}
\mathbf{u}_{e} & =\left[\mathbf{N}_{\mathbf{u}}\right]^{t}\left\{\mathbf{U}_{e}\right\} \\
\phi_{e} & =\left[\mathbf{N}_{\phi}\right]^{t}\left\{\boldsymbol{\phi}_{e}\right\}
\end{aligned}
$$

onde:

$$
\begin{aligned}
& {\left[\mathbf{N}_{\mathbf{u}}\right]=\text { função de forma de deslocamento mecânico }} \\
& {\left[\mathbf{N}_{\phi}\right]=\text { função de forma de deslocamento elétrico }} \\
& \left\{\mathbf{U}_{e}\right\}=\text { vetor deslocamento nodal } \\
& \left\{\phi_{e}\right\}=\text { vetor potencial elétrico nodal } \\
& \mathbf{u}_{e}=\text { deslocamento do elemento } \\
& \phi_{e} \quad=\text { potencial elétrico no elemento }
\end{aligned}
$$

e são dados por:

$$
\begin{aligned}
{\left[\mathbf{N}_{\mathbf{u}}\right] } & =\left[\begin{array}{cccccccc}
N_{1} & 0 & N_{2} & 0 & N_{3} & 0 & N_{4} & 0 \\
0 & N_{1} & 0 & N_{2} & 0 & N_{3} & 0 & N_{4}
\end{array}\right] \\
{\left[\mathbf{N}_{\phi}\right] } & =\left[\begin{array}{lllll}
N_{1} & N_{2} & N_{3} & N_{4}
\end{array}\right] \\
\left\{\mathbf{U}_{e}\right\} & =\left\{\begin{array}{llllllll}
u_{1} & w_{1} & u_{2} & w_{2} & u_{3} & w_{3} & u_{4} & w_{4}
\end{array}\right\}^{t} \\
\left\{\boldsymbol{\phi}_{e}\right\} & =\left\{\begin{array}{llll}
\phi_{1} & \phi_{2} & \phi_{3} & \phi_{4}
\end{array}\right\}^{t} \\
\mathbf{u}_{e} & =\left\{\begin{array}{ll}
u_{e} & w_{e}
\end{array}\right\}^{t}
\end{aligned}
$$

A função de forma são funções de interpolação de Lagrange e são expressas em coordenadas $(\xi, \eta)$ :

$$
\begin{array}{lll}
N_{1}(\xi, \eta)=\frac{(1-\xi)(1-\eta)}{4} & ; & N_{2}(\xi, \eta)=\frac{(1+\xi)(1-\eta)}{4} \\
N_{3}(\xi, \eta)=\frac{(1+\xi)(1+\eta)}{4} & ; & N_{4}(\xi, \eta)=\frac{(1-\xi)(1+\eta)}{4}
\end{array}
$$

Considerando as Eqs. (2.1) e (2.4), o vetor deformações $\mathbf{S}$ e o vetor campo elétrico $\mathbf{E}$ são relacionados com os deslocamentos mecânicos e potenciais elétricos nodais da seguinte 
maneira:

$$
\mathbf{S}^{\text {Axissimétrico }}=\left[\begin{array}{c}
\frac{\partial u}{\partial r} \\
\frac{\partial w}{\partial z} \\
\frac{u}{r} \\
\frac{\partial u}{\partial z}+\frac{\partial w}{\partial r}
\end{array}\right]
$$

para o elemento axissimétrico, sendo

$$
r=\sum_{i=1}^{4} r_{i} N_{i}, \quad z=\sum_{i=1}^{4} z_{i} N_{i}
$$

onde $r_{i}$ é a coordenada de cada nó do elemento. E para o elemento EPTM:

$$
\mathbf{S}^{E P T M}=\left[\begin{array}{c}
\frac{\partial u}{\partial x} \\
\frac{\partial w}{\partial z} \\
\frac{\partial u}{\partial z}+\frac{\partial w}{\partial x}
\end{array}\right]
$$

O potencial elétrico é dado por:

$$
\mathbf{E}=-\nabla \phi
$$

que, para o elemento axissimétrico fica:

$$
\mathbf{E}^{\text {Axissimétrico }}=-\left\{\begin{array}{c}
\frac{\partial \phi}{\partial r} \\
\frac{\partial \phi}{\partial z}
\end{array}\right\}
$$

e para o elemento EPTM fica:

$$
\mathbf{E}^{E P T M}=-\left\{\begin{array}{l}
\frac{\partial \phi}{\partial x} \\
\frac{\partial \phi}{\partial z}
\end{array}\right\}
$$

Como o MEF está sendo considerado no plano, as coordenadas cartesianas $(x, z)$ são utilizadas ao invés das coordenadas polares $(r, z)$, como pode ser visto na Fig. 2.5.

Então pode-se reescrever a Eq. (2.18) da seguinte maneira:

$$
r=x=\sum_{i=1}^{4} x_{i} N_{i} \quad, \quad z=\sum_{i=1}^{4} z_{i} N_{i}
$$




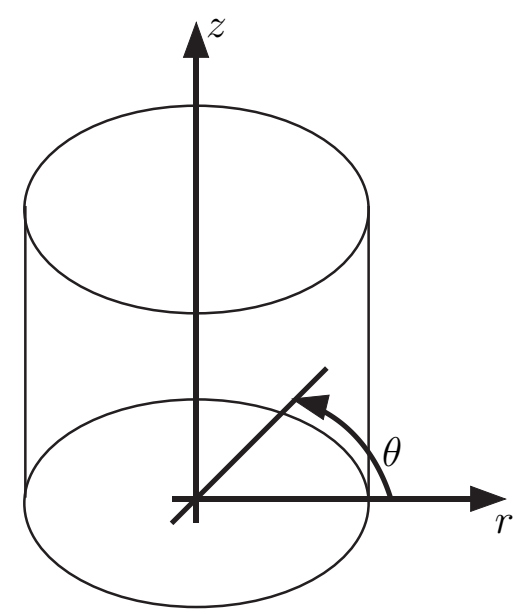

(a) Coordenadas Polares

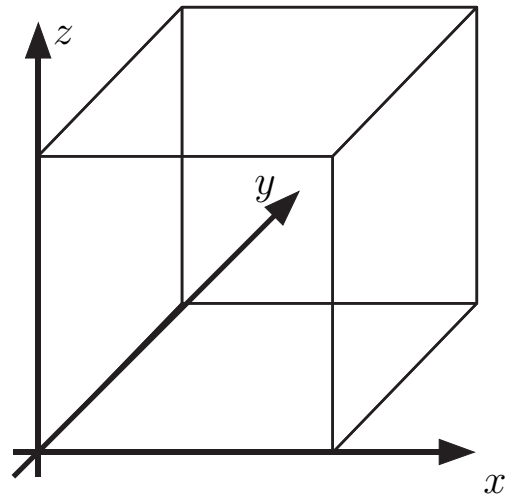

(b) Coordenadas Cartesianas

Figura 2.5: Equivalência entre as coordenadas polares e cartesianas.

e com isso as Eqs. (2.17) e (2.19) ficam:

$$
\mathbf{S}^{\text {Axissimétrico }}=\left[\begin{array}{c}
\frac{\partial u}{\partial x} \\
\frac{\partial w}{\partial z} \\
\frac{u}{x} \\
\frac{\partial u}{\partial z}+\frac{\partial w}{\partial x}
\end{array}\right], \mathbf{S}^{E P T M}=\left[\begin{array}{c}
\frac{\partial u}{\partial x} \\
\frac{\partial w}{\partial z} \\
\frac{\partial u}{\partial z}+\frac{\partial w}{\partial x}
\end{array}\right]
$$

e as Eqs. (2.21) e (2.22) ficam iguais e podem ser expressas como:

$$
\mathbf{E}=-\left\{\begin{array}{l}
\frac{\partial \phi}{\partial x} \\
\frac{\partial \phi}{\partial z}
\end{array}\right\}
$$

Considerando as Eqs. (2.9) e (2.10), pode-se reescrever as Eqs. (2.24) e (2.25) como:

$$
\begin{gathered}
\mathbf{S}^{\text {Axissimétrico }}=\left[\begin{array}{cc}
\frac{\partial}{\partial x} & 0 \\
0 & \frac{\partial}{\partial z} \\
\frac{1}{x} & 0 \\
\frac{\partial}{\partial z} & \frac{\partial}{\partial x}
\end{array}\right]\left[\mathbf{N}_{\mathbf{u}}\right]\left\{\mathbf{U}_{e}\right\} \quad, \mathbf{S}^{E P T M}=\left[\begin{array}{cc}
\frac{\partial}{\partial x} & 0 \\
0 & \frac{\partial}{\partial z} \\
\frac{\partial}{\partial z} & \frac{\partial}{\partial x}
\end{array}\right]\left[\mathbf{N}_{\mathbf{u}}\right]\left\{\mathbf{U}_{e}\right\} \\
\mathbf{E}=-\left[\begin{array}{c}
\frac{\partial}{\partial x} \\
\frac{\partial}{\partial z}
\end{array}\right]\left[\mathbf{N}_{\phi}\right]\left\{\boldsymbol{\phi}_{e}\right\}
\end{gathered}
$$


Para simplificação de notação, pode-se assumir que:

$$
\begin{gathered}
\mathbf{B}_{\mathbf{u}}^{\text {Axissimétrico }}=\left[\begin{array}{cc}
\frac{\partial}{\partial x} & 0 \\
0 & \frac{\partial}{\partial z} \\
\frac{1}{x} & 0 \\
\frac{\partial}{\partial z} & \frac{\partial}{\partial x}
\end{array}\right]\left[\mathbf{N}_{\mathbf{u}}\right], \mathbf{B}_{\mathbf{u}}{ }^{E P T M}=\left[\begin{array}{cc}
\frac{\partial}{\partial x} & 0 \\
0 & \frac{\partial}{\partial z} \\
\frac{\partial}{\partial z} & \frac{\partial}{\partial x}
\end{array}\right]\left[\mathbf{N}_{\mathbf{u}}\right] \\
\mathbf{B}_{\phi}=\left[\begin{array}{c}
\frac{\partial}{\partial x} \\
\frac{\partial}{\partial z}
\end{array}\right]\left[\mathbf{N}_{\phi}\right]
\end{gathered}
$$

Então, a Eq. (2.26) pode ser reescrita da seguinte maneira:

$$
\begin{gathered}
\mathbf{S}^{\text {Axissimétrico }}=\left[\mathbf{B}_{\mathbf{u}}{ }^{\text {Axissimétrico }}\right]\left\{\mathbf{U}_{e}\right\} ; \mathbf{S}^{E P T M}=\left[\mathbf{B}_{\mathbf{u}}{ }^{\text {EPTM }}\right]\left\{\mathbf{U}_{e}\right\} \\
\mathbf{E}=-\left[\mathbf{B}_{\phi}\right]\left\{\boldsymbol{\phi}_{e}\right\}
\end{gathered}
$$

Os diferenciais $(d x, d z)$ da expressão acima são relacionados com os diferenciais $(d \xi, d \eta)$ por meio da Eq. (2.7), então:

$$
d x=\frac{\partial x}{\partial \xi} d \xi+\frac{\partial x}{\partial \eta} d \eta, d z=\frac{\partial z}{\partial \xi} d \xi+\frac{\partial z}{\partial \eta} d \eta
$$

onde

$$
\begin{array}{ll}
\frac{\partial x}{\partial \xi}=\sum_{i=1}^{4} \frac{\partial N_{i}}{\partial \xi} x_{i} & , \quad \frac{\partial z}{\partial \xi}=\sum_{i=1}^{4} \frac{\partial N_{i}}{\partial \xi} z_{i} \\
\frac{\partial x}{\partial \eta}=\sum_{i=1}^{4} \frac{\partial N_{i}}{\partial \eta} x_{i} & , \quad \frac{\partial z}{\partial \eta}=\sum_{i=1}^{4} \frac{\partial N_{i}}{\partial \eta} z_{i}
\end{array}
$$

As derivadas das coordenadas são combinadas na forma matricial e dadas por:

$$
[\mathbf{J}]=\left[\begin{array}{ll}
\frac{\partial x}{\partial \xi} & \frac{\partial z}{\partial \xi} \\
\frac{\partial x}{\partial \eta} & \frac{\partial z}{\partial \eta}
\end{array}\right]
$$

onde $[\mathbf{J}]$ é a matriz Jacobiana de transformação de coordenadas. Relacionando as Eqs. (2.29) e (2.31), os diferenciais de dois sistemas de coordenadas são dados por:

$$
\left\{\begin{array}{l}
d x \\
d z
\end{array}\right\}=[\mathbf{J}]^{t}\left\{\begin{array}{l}
d \xi \\
d \eta
\end{array}\right\}
$$

De maneira análoga, as derivadas das funções de forma dos nós $i$ são relacionadas por:

$$
\left\{\begin{array}{c}
\frac{\partial N_{i}}{\partial x} \\
\frac{\partial N_{i}}{\partial z}
\end{array}\right\}=[\mathbf{J}]^{-1}\left\{\begin{array}{c}
\frac{\partial N_{i}}{\partial \xi} \\
\frac{\partial N_{i}}{\partial \eta}
\end{array}\right\}
$$




\subsection{Formulação Matricial do Elemento}

Definida a formulação dos graus de liberdade o elemento isoparamétrico, pode-se definir a formulação matricial do elemento. As deformações mecânicas e o campo elétrico Eq. (2.28) em função dos deslocamentos virtuais serão dadas por:

$$
\begin{gathered}
\left\{\delta \mathbf{S}^{\text {Axissimétrico }}\right\}=\left[\mathbf{B}_{\mathbf{u}}{ }^{\text {Axissimétrico }}\right]\left\{\delta \mathbf{U}_{e}\right\} ;\left\{\delta \mathbf{S}^{E P T M}\right\}=\left[\mathbf{B}_{\mathbf{u}}{ }^{\text {EPTM }}\right]\left\{\delta \mathbf{U}_{e}\right\} \\
\{\delta \mathbf{E}\}=-\left[\mathbf{B}_{\phi}\right]\left\{\delta \boldsymbol{\phi}_{e}\right\}
\end{gathered}
$$

Substituindo as Eqs. (2.28) e (2.34) nas Eqs. (B.13) e (B.14), pode-se escrever (sabendo-se que: $\mathbf{E}=-\nabla \phi)$ :

$$
\begin{aligned}
& \left.\left\{\delta \mathbf{U}_{e}\right\}^{t} \int_{V_{e}}\left(\left[\rho\left[\mathbf{N}_{\mathbf{u}}\right]^{t}\left[\mathbf{N}_{\mathbf{u}}\right]\left\{\ddot{\mathbf{U}}_{e}\right\}\right]+\left[\mathbf{B}_{\mathbf{u}}\right]^{t}\left[\mathbf{c}^{E}\right]\left[\mathbf{B}_{\mathbf{u}}\right]\left\{\mathbf{U}_{e}\right\}+\left[\mathbf{B}_{\mathbf{u}}\right]^{t}[\mathbf{e}]^{t}\left[\mathbf{B}_{\phi}\right]\left\{\boldsymbol{\phi}_{e}\right\}\right) d V \overline{\bar{F}}\right) \\
& =\left\{\delta \mathbf{U}_{e}\right\}^{t}\left[\mathbf{N}_{\mathbf{u}}\right]^{t}\left\{\mathbf{f}_{e}\right\} \\
& \left\{\delta \boldsymbol{\phi}_{e}\right\}^{t} \int_{V_{e}}\left(\left[\mathbf{B}_{\phi}\right]^{t}[\mathbf{e}]\left[\mathbf{B}_{\mathbf{u}}\right]\left\{\mathbf{U}_{e}\right\}-\left[\mathbf{B}_{\phi}\right]^{t}\left[\varepsilon^{S}\right]\left[\mathbf{B}_{\phi}\right]\left\{\boldsymbol{\phi}_{e}\right\}\right) d V= \\
& =\left\{\delta \boldsymbol{\phi}_{e}\right\}^{t}\left[\mathbf{N}_{\phi}\right]^{t}\left\{\mathbf{q}_{e}\right\}
\end{aligned}
$$

desde que qualquer valor admissível dos deslocamentos $\left\{\delta \mathbf{U}_{e}\right\}$ e potenciais elétricos $\left\{\delta \phi_{e}\right\}$ sejam compatíveis com as condições de contorno, onde $\left\{\mathbf{f}_{e}\right\}$ e $\left\{\mathbf{q}_{e}\right\}$ denotam os carregamentos mecânicos e cargas elétricos, internos e externos, de cada elemento.

Então, fazendo:

$$
\begin{aligned}
& \{\mathbf{F}\}_{e}=\left[\mathbf{N}_{\mathbf{u}}\right]^{t}\left\{\mathbf{f}_{e}\right\}=\left[\mathbf{N}_{\mathbf{u}}\right]^{t}\left[\left\{\mathbf{f}_{\text {ext }}\right\}+\left\{\mathbf{f}_{\text {int }}\right\}\right]=\left\{\mathbf{F}_{\text {ext }}\right\}+\left\{\mathbf{F}_{\text {int }}\right\} \\
& \{\mathbf{Q}\}_{e}=\left[\mathbf{N}_{\phi}\right]^{t}\left\{\mathbf{q}_{e}\right\}=\left[\mathbf{N}_{\phi}\right]^{t}\left[\left\{\mathbf{q}_{\text {ext }}\right\}+\left\{\mathbf{q}_{\text {int }}\right\}\right]=\left\{\mathbf{Q}_{\text {ext }}\right\}+\left\{\mathbf{Q}_{\text {int }}\right\}
\end{aligned}
$$

pode-se escrever, para cada elemento finito, as Eqs. (2.35) e (2.36) na forma matricial da seguinte maneira:

$$
\begin{aligned}
{\left[\mathbf{M}_{\mathbf{u u}}\right]_{e}\left\{\ddot{\mathbf{U}}_{e}\right\}+\left[\mathbf{K}_{\mathbf{u u}}\right]_{e}\left\{\mathbf{U}_{e}\right\}+\left[\mathbf{K}_{\mathbf{u} \phi}\right]_{e}\left\{\boldsymbol{\phi}_{e}\right\} } & =\{\mathbf{F}\}_{e} \\
{\left[\mathbf{K}_{\mathbf{u} \phi}\right]_{e}^{t}\left\{\mathbf{U}_{e}\right\}-\left[\mathbf{K}_{\phi \phi}\right]_{e}\left\{\boldsymbol{\phi}_{e}\right\} } & =\{\mathbf{Q}\}_{e}
\end{aligned}
$$


Integrando no volume, obtém-se as seguintes matrizes:

$$
\begin{aligned}
{\left[\mathbf{M}_{\mathbf{u u}}\right]_{e} } & =\int_{V_{e}} \rho\left[\mathbf{N}_{\mathbf{u}}\right]^{t}\left[\mathbf{N}_{\mathbf{u}}\right] d V_{e} \\
{\left[\mathbf{K}_{\mathbf{u u}}\right]_{e} } & =\int_{V_{e}}\left[\mathbf{B}_{\mathbf{u}}\right]^{t}\left[\mathbf{c}^{E}\right]\left[\mathbf{B}_{\mathbf{u}}\right] d V_{e} \\
{\left[\mathbf{K}_{\mathbf{u} \phi}\right]_{e} } & =\int_{V_{e}}\left[\mathbf{B}_{\mathbf{u}}\right]^{t}[\mathbf{e}]^{t}\left[\mathbf{B}_{\phi}\right] d V_{e} \\
{\left[\mathbf{K}_{\phi \phi}\right]_{e} } & =\int_{V_{e}}\left[\mathbf{B}_{\phi}\right]^{t}\left[\varepsilon^{S}\right]\left[\mathbf{B}_{\phi}\right] d V_{e}
\end{aligned}
$$

onde $\left[\mathbf{M}_{\mathbf{u u}}\right]_{e},\left[\mathbf{K}_{\mathbf{u u}}\right]_{e},\left[\mathbf{K}_{\mathbf{u} \phi}\right]_{e}$ e $\left[\mathbf{K}_{\phi \phi}\right]_{e}$ são matrizes de massa, rigidez elástica, piezelétrica e dielétrica do elemento, respectivamente. Finalmente, através do Jacobiano (Eq. (2.31)) pode-se transformar as coordenadas locais em coordenadas globais e, considerando que as análises são feitas para elementos axissimétricos ou EPTM, as Eqs. (2.40) à (2.43) podem ser escritas da seguinte forma:

$$
\begin{aligned}
& {\left[\mathbf{M}_{\mathbf{u u}]_{e}}=2 \pi \int_{-1}^{1} \int_{-1}^{1} \rho\left[\mathbf{N}_{\mathbf{u}}\right]^{t}\left[\mathbf{N}_{\mathbf{u}}\right] r \operatorname{det}[\mathbf{J}] d \eta d \xi\right.} \\
& {\left[\mathbf{K}_{\mathbf{u u}]_{e}}=2 \pi \int_{-1}^{1} \int_{-1}^{1}\left[\mathbf{B}_{\mathbf{u}}\right]^{t}\left[\mathbf{c}^{E}\right]\left[\mathbf{B}_{\mathbf{u}}\right] r \operatorname{det}[\mathbf{J}] d \eta d \xi\right.} \\
& {\left[\mathbf{K}_{\mathbf{u} \phi}\right]_{e}=2 \pi \int_{-1}^{1} \int_{-1}^{1}\left[\mathbf{B}_{\mathbf{u}}\right]^{t}[\mathbf{e}]^{t}\left[\mathbf{B}_{\phi}\right] r \operatorname{det}[\mathbf{J}] d \eta d \xi} \\
& {\left[\mathbf{K}_{\phi \phi}\right]_{e}=2 \pi \int_{-1}^{1} \int_{-1}^{1}\left[\mathbf{B}_{\phi}\right]^{t}\left[\boldsymbol{\varepsilon}^{S}\right]\left[\mathbf{B}_{\phi}\right] r \operatorname{det}[\mathbf{J}] d \eta d \xi}
\end{aligned}
$$

para o elemento axissimétrico, onde $r$ é o raio do elemento finito, dado pela Eq. (2.23):

$$
r=x=\sum_{i=1}^{4} x_{i} N_{i}
$$

sendo que $x_{i}$ é a coordenada na direção 1 de cada nó e $N_{i}$ é a função de forma correspondente a cada nó. Para o elemento EPTM tem-se:

$$
\begin{aligned}
& {\left[\mathbf{M}_{\mathbf{u u}}\right]_{e}=h_{e} \int_{-1}^{1} \int_{-1}^{1} \rho\left[\mathbf{N}_{\mathbf{u}}\right]^{t}\left[\mathbf{N}_{\mathbf{u}}\right] \operatorname{det}[\mathbf{J}] d \eta d \xi} \\
& {\left[\mathbf{K}_{\mathbf{u u}}\right]_{e}=h_{e} \int_{-1}^{1} \int_{-1}^{1}\left[\mathbf{B}_{\mathbf{u}}\right]^{t}\left[\mathbf{c}^{E}\right]\left[\mathbf{B}_{\mathbf{u}}\right] \operatorname{det}[\mathbf{J}] d \eta d \xi} \\
& {\left[\mathbf{K}_{\mathbf{u} \phi}\right]_{e}=h_{e} \int_{-1}^{1} \int_{-1}^{1}\left[\mathbf{B}_{\mathbf{u}}\right]^{t}[\mathbf{e}]^{t}\left[\mathbf{B}_{\phi}\right] \operatorname{det}[\mathbf{J}] d \eta d \xi} \\
& {\left[\mathbf{K}_{\phi \phi}\right]_{e}=h_{e} \int_{-1}^{1} \int_{-1}^{1}\left[\mathbf{B}_{\phi}\right]^{t}\left[\boldsymbol{\varepsilon}^{S}\right]\left[\mathbf{B}_{\phi}\right] \operatorname{det}[\mathbf{J}] d \eta d \xi}
\end{aligned}
$$

onde $h_{e}$ é a espessura do elemento finito. 
Assim, combinando-se as Eqs. (2.38) e (2.39) a equação linear dinâmica do MEF piezelétrico para cada elemento, tanto axissimétrico quanto EPTM, é dada por:

$$
\left[\begin{array}{cc}
\mathbf{M}_{\mathbf{u u}} & 0 \\
0 & 0
\end{array}\right]_{e}\left\{\begin{array}{l}
\ddot{\mathbf{U}}_{e} \\
\ddot{\phi}_{e}
\end{array}\right\}+\left[\begin{array}{ll}
\mathbf{K}_{\mathbf{u u}} & \mathbf{K}_{\mathbf{u} \phi} \\
\mathbf{K}_{\mathbf{u} \phi}^{t} & \mathbf{K}_{\phi \phi}
\end{array}\right]_{e}\left\{\begin{array}{c}
\mathbf{U}_{e} \\
\boldsymbol{\phi}_{e}
\end{array}\right\}=\left\{\begin{array}{l}
\mathbf{F} \\
\mathbf{Q}
\end{array}\right\}_{e}
$$

A equação acima representa a matriz local de um elemento piezelétrico, de tamanho $12 \times 12$ (para o elemento não-piezelétrico, o tamanho da matriz é 8x8). Na forma global, a Eq. (2.53) fica:

$$
\left[\begin{array}{cc}
\mathbf{M}_{\mathbf{u u}} & 0 \\
0 & 0
\end{array}\right]\left\{\begin{array}{l}
\ddot{\mathbf{U}} \\
\ddot{\boldsymbol{\phi}}
\end{array}\right\}+\left[\begin{array}{ll}
\mathbf{K}_{\mathbf{u u}} & \mathbf{K}_{\mathbf{u} \phi} \\
\mathbf{K}_{\mathbf{u} \phi}^{t} & \mathbf{K}_{\phi \phi}
\end{array}\right]\left\{\begin{array}{l}
\mathbf{U} \\
\boldsymbol{\phi}
\end{array}\right\}=\left\{\begin{array}{l}
\mathbf{F} \\
\mathbf{Q}
\end{array}\right\}
$$

Considerando que o deslocamento mecânico e o potencial elétrico em cada ponto são funções oscilatórias no tempo $t$, se não há amortecimento, pode-se representar o deslocamento mecânico e o potencial elétrico como funções seno, ou seja:

$$
\begin{aligned}
& \mathbf{U}=\mathbf{A} \sin \omega t \Rightarrow \dot{\mathbf{U}}=\mathbf{A} \omega \cos \omega t \Rightarrow \ddot{\mathbf{U}}=-\omega^{2} \mathbf{A} \sin \omega t=-\omega^{2} \mathbf{U} \\
& \phi=\mathbf{A} \sin \omega t \Rightarrow \dot{\boldsymbol{\phi}}=\mathbf{A} \omega \cos \omega t \Rightarrow \ddot{\boldsymbol{\phi}}=-\omega^{2} \mathbf{A} \sin \omega t=-\omega^{2} \boldsymbol{\phi}
\end{aligned}
$$

onde A é o vetor de amplitudes de oscilação e $\omega$ é a freqüência de operação, e considerando as seguintes simplificações matriciais:

$$
[\mathbf{M}]=\left[\begin{array}{cc}
\mathbf{M}_{\mathbf{u u}} & 0 \\
0 & 0
\end{array}\right],[\mathbf{K}]=\left[\begin{array}{ll}
\mathbf{K}_{\mathbf{u u}} & \mathbf{K}_{\mathbf{u} \phi} \\
\mathbf{K}_{\mathbf{u} \phi}^{t} & \mathbf{K}_{\phi \phi}
\end{array}\right],\{\boldsymbol{\Gamma}\}=\left\{\begin{array}{l}
\mathbf{F} \\
\mathbf{Q}
\end{array}\right\},\{\boldsymbol{\Psi}\}=\left\{\begin{array}{c}
\mathbf{U} \\
\boldsymbol{\phi}
\end{array}\right\}
$$

a Eq. (2.54) pode ser reescrita como:

$$
\left([\mathbf{K}]-\omega^{2}[\mathbf{M}]\right)\{\boldsymbol{\Psi}\}=\{\boldsymbol{\Gamma}\}
$$

e fazendo:

$$
[\boldsymbol{\kappa}]=\left([\mathbf{K}]-\omega^{2}[\mathbf{M}]\right)
$$

tem-se:

$$
[\boldsymbol{\kappa}]\{\Psi\}=\{\boldsymbol{\Gamma}\}
$$

A solução da Eq. (2.59) utilizando excitação por potencial elétrico é dada no Apêndice C. 


\subsection{Cálculo de Autovalores e Autovetores}

Para problemas vibracionais, ou seja, problemas que consideram a situação dinâmica da estrutura, o maior desafio que o engenheiro encontra está relacionado com as freqüências de ressonância. A ressonância é um fenômeno no qual a estrutura acumula a menor quantidade de energia, possibilitando que certos pontos vibrem com máxima amplitude, porém, existindo a possibilidade de pontos estáticos (amplitude nula), comprometendo assim, a uniformização dos deslocamentos.

Os dois tipos de freqüências que caracterizam o comportamento do transdutor piezelétrico para diferentes modos de vibrar são as freqüências de ressonância $\left(\omega_{r}\right)$ e anti-ressonância $\left(\omega_{a}\right)$ piezelétricas. Essas freqüências estão relacionadas com a impedância elétrica do transdutor, definida como a razão entre a tensão e a corrente elétrica nos eletrodos do transdutor (NAILLON; COURSANT; BESNIER, 1983; LERCH, 1990). As freqüências de ressonância caracterizam-se por apresentar impedância mínima, são excitadas por pulso de potencial elétrico e são obtidas com os eletrodos das cerâmicas curto-circuitados. Já as freqüências de anti-ressonância caracterizam-se por apresentar impedância máxima, são excitadas por pulso de carga elétrica e são obtidas na condição de circuito aberto.

Utilizando o MEF, essas freqüências podem ser obtidas resolvendo dois problemas de autovalores gerados por dois diferentes casos de condições de contorno aplicadas à Eq. (2.59), considerando $\mathbf{F}=0$ (GUO; CAWLEY; HITChings, 1992). Para a freqüência de ressonância considera-se que os eletrodos estão curto-circuitados, ou seja, os graus de liberdade (GDLs) referentes ao potencial elétrico em todos os eletrodos devem ser iguais a zero $\left(\phi_{\text {eletrodos }}=0\right)$ na Eq. (2.59). Para a freqüência de anti-ressonância o transdutor deve estar em circuito aberto, ou seja, as cargas elétricas devem ser iguais a zero nos eletrodos $(\mathbf{Q}=0)$.

No modelo de MEF do transdutor com quatro cerâmicas (Fig. 2.6), considerando que os eletrodos 1, 3 e 5 estão sempre aterrados $\left(\phi_{\text {eletrodos }}=0\right)$, os dois problemas de autovalor podem ser obtidos simplesmente mudando a condição de contorno elétrica nos eletrodos 2 e 4, ou seja, o potencial elétrico nos graus de liberdade é zero $\left(\phi_{\text {eletrodos }}=0\right)$ para a freqüência $\omega_{r}$, e para a freqüência $\omega_{a}$ os GDLs são restringidos para serem equipotenciais e considera-se Q = 0 (GUO; CAWLEY; HITCHINGS, 1992; NAILLON; COURSANT; BESNIER, 1983).

Geralmente, freqüências de ressonância e anti-ressonância de modos de vibrar 


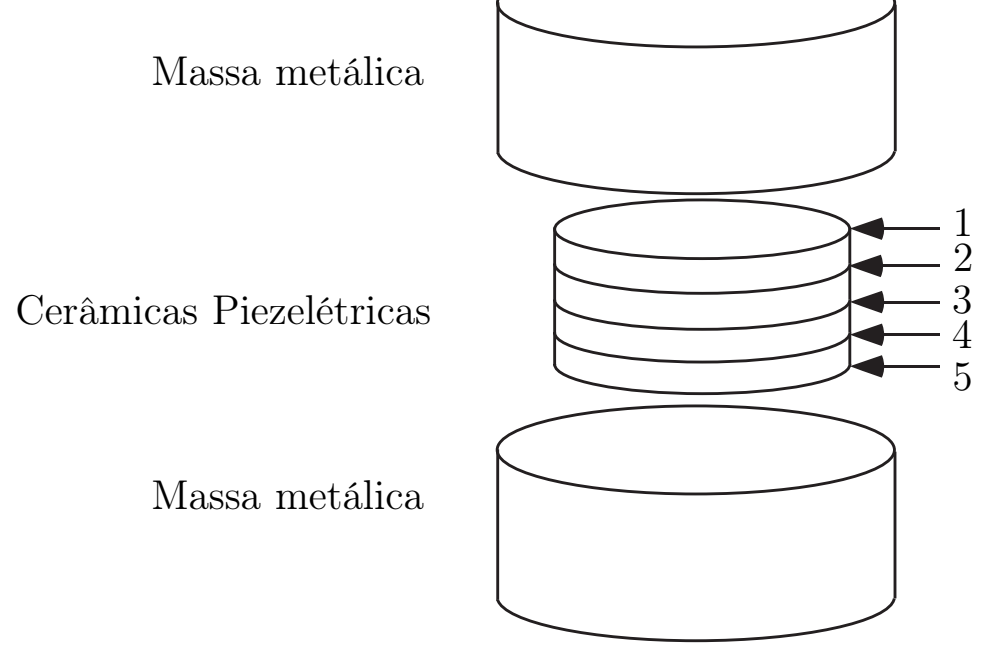

Figura 2.6: Representação esquemática de um transdutor piezelétrico expandido.

correspondentes se apresentam bem próximas entre si (HIROSE et al., 1996). No entanto, existem modos que apresentam uma diferença maior entre esses dois tipos de freqüências, comparadas com os outros modos. Esses modos em particular são chamados de modos piezelétricos, que neste trabalho, é o caso da freqüência do modo de pistão que se deseja otimizar. Esses modos piezelétricos são chamados de modos excitáveis, pois possuem o valor do coeficiente de acoplamento eletromecânico maior do que os outros modos, permitindo que mais energia elétrica se transforme em energia mecânica, ou vice-e-versa. O coeficiente de acoplamento eletromecânico pode ser calculado da seguinte maneira:

$$
k^{2}=\frac{\omega_{a}^{2}-\omega_{r}^{2}}{\omega_{a}^{2}}
$$

onde $\omega_{a}$ e $\omega_{r}$ são as freqüências de anti-ressonância e ressonância respectivamente. Então, quanto maior for a diferença entre essas duas freqüências, maior será o coeficiente de acoplamento eletromecânico.

Existem dois meios mais utilizados para se encontrar as freqüências de ressonância e anti-ressonância: levantando a curva de impedância da estrutura, ou calculando os dois tipos de freqüências para cada condição de contorno. Pelo MEF a segunda opção é mais eficiente, pois para levantar a curva de impedância, é necessária que seja feita uma análise harmônica dentro de uma faixa de freqüências, e para que se possa obter as freqüências de ressonância e anti-ressonância com precisão de algumas casas decimais, é necessária uma boa discretização na freqüência, o que aumenta o custo computacional pois aumenta o número de iterações.

Contudo, os cálculos das freqüências de ressonância e anti-ressonância devem ser feitos 
separadamente, como já dito anteriormente, o que duplica o custo computacional e o tempo de processamento, comparando com o caso em que é necessário apenas o cálculo das freqüências de ressonância.

Então, para eliminar a necessidade de se calcular as freqüências de ressonância e anti-ressonância a fim de se encontrar o modo que possui maior coeficiente de acoplamento eletromecânico, é utilizada uma formulação que procura o modo desejado dentre aqueles calculados. A formulação utilizada se baseia no método "Modal Assurance Criterion" (MAC), que está explicado na Seção 2.6. Assim, dado que já se conhece o modo com o maior coeficiente de acoplamento eletromecânico, a formulação "Modal Assurance Criterion" (MAC) irá procurá-lo dentre os modos calculados.

Por isso, neste trabalho, apenas as freqüências de ressonância são calculadas resolvendo-se os problemas de autovalores resultantes da aplicação das condições de contorno à Eq. (2.54):

$$
\left(\left[\begin{array}{ll}
\overline{\mathbf{K}}_{\mathbf{u u}} & \overline{\mathbf{K}}_{\mathbf{u} \phi} \\
\overline{\mathbf{K}}_{\mathbf{u} \phi}^{t} & \overline{\mathbf{K}}_{\phi \phi}
\end{array}\right]-\lambda\left[\begin{array}{cc}
\overline{\mathbf{M}}_{\mathbf{u u}} & 0 \\
0 & 0
\end{array}\right]\right)\left\{\begin{array}{l}
\mathbf{W}_{\mathbf{u}} \\
\mathbf{W}_{\phi}
\end{array}\right\}=\left\{\begin{array}{c}
0 \\
0
\end{array}\right\} ; \lambda=\omega^{2}
$$

onde $\lambda$ é um autovalor, $\left\{\mathbf{W}_{\mathbf{u}}\right\}$ é um autovetor correspondente aos graus de liberdade mecânicos e $\left\{\mathbf{W}_{\phi}\right\}$ é um autovetor correspondente aos graus de liberdade elétricos. $\left[\overline{\mathbf{K}}_{\mathbf{u u}}\right],\left[\overline{\mathbf{K}}_{\mathbf{u} \phi}\right]$ e $\left[\overline{\mathbf{K}}_{\phi \phi}\right]$ são as matrizes de rigidez mecânica, dielétrica e piezelétrica respectivamente, considerando as condições de contorno para o cálculo das freqüências de ressonância. $\left[\overline{\mathbf{M}}_{\mathbf{u u}}\right]$ é a matriz de massa mecânica, também considerando as condições de contorno para o cálculo das freqüências de ressonância.

A Eq. (2.61) pode ser simplificada da seguinte maneira:

$$
[\overline{\mathbf{K}}]\{\mathbf{W}\}=\lambda[\overline{\mathbf{M}}]\{\mathbf{W}\}
$$

onde

$$
[\overline{\mathbf{K}}]=\left[\begin{array}{cc}
\overline{\mathbf{K}}_{\mathbf{u u}} & \overline{\mathbf{K}}_{\mathbf{u} \phi} \\
\overline{\mathbf{K}}_{\mathbf{u} \phi}^{t} & \overline{\mathbf{K}}_{\phi \phi}
\end{array}\right],[\overline{\mathbf{M}}]=\left[\begin{array}{cc}
\overline{\mathbf{M}}_{\mathbf{u u}} & 0 \\
0 & 0
\end{array}\right],\{\mathbf{W}\}=\left\{\begin{array}{c}
\mathbf{W}_{\mathbf{u}} \\
\mathbf{W}_{\phi}
\end{array}\right\}
$$

Existem diversos meios de se resolver este problema de autovalores. O método mais utilizado na literatura é resolver o sistema de equações da Eq. (2.61) utilizando o método de Lanczos. A vantagem deste método é que a banda da matriz de rigidez total do modelo, envolvendo os termos elásticos, piezelétricos e dielétricos, são preservados, reduzindo o espaço de armazenagem necessário, levando à obtenção de um resultado mais confiável. 
Contudo, deve-se lidar com a matriz de rigidez total que é indefinida (autovalores positivos e negativos) devido aos termos negativos da diagonal da parte dielétrica da matriz. Como conseqüência, métodos diretos que utilizam solucionadores tradicionais como o método de iterações por subespaço não funcionam e por isso, o método de Lanczos é o mais utilizado. Deve-se também lidar com a matriz de massa que é positiva semi-definida (por causa dos termos iguais a zero relacionados aos GDLs elétricos), e para isso, Yong (1995), Yong e Cho (1994) propuseram um esquema de armazenagem para a matriz de massa, onde fatora-se apenas os termos mecânicos $\left[\overline{\mathbf{M}}_{\mathbf{u u}}\right]$ utilizando a fatoração Cholesky:

$$
\left[\overline{\mathbf{M}}_{\mathbf{u u}}\right]=\left[\mathbf{L}_{\mu}\right]\left[\mathbf{L}_{\mu}\right]^{T}
$$

e então

$$
[\overline{\mathbf{M}}]=\left[\begin{array}{cc}
\mathbf{L}_{\mu} & 0 \\
0 & 0
\end{array}\right]\left[\begin{array}{cc}
\mathbf{L}_{\mu}^{T} & 0 \\
0 & 0
\end{array}\right]
$$

Já a matriz de rigidez é fatorada da seguinte maneira:

$$
[\overline{\mathbf{K}}]=[\mathbf{L}][\mathbf{D}][\mathbf{L}]^{T}
$$

Assim, pode-se reescrever a Eq. (2.62):

$$
\frac{1}{\lambda}[\mathbf{L}][\mathbf{D}][\mathbf{L}]^{T}\{\mathbf{W}\}=\left[\begin{array}{cc}
\mathbf{L}_{\mu} & 0 \\
0 & 0
\end{array}\right]\left[\begin{array}{cc}
\mathbf{L}_{\mu} & 0 \\
0 & 0
\end{array}\right]^{T}\{\mathbf{W}\}
$$

que ainda pode ser transformado num problema típico de autovalor na forma:

$$
\frac{1}{\lambda}\{\mathbf{V}\}=[\mathbf{A}]\{\mathbf{V}\}
$$

onde

$$
\begin{gathered}
\{\mathbf{V}\}=\left[\begin{array}{cc}
\mathbf{L}_{\mu} & 0 \\
0 & 0
\end{array}\right]^{T}\{\mathbf{W}\} \\
{[\mathbf{A}]=\left[\begin{array}{cc}
\mathbf{L}_{\mu} & 0 \\
0 & 0
\end{array}\right]^{T}[\mathbf{L}]^{-T}[\mathbf{D}]^{-1}[\mathbf{L}]^{-1}\left[\begin{array}{cc}
\mathbf{L}_{\mu} & 0 \\
0 & 0
\end{array}\right]}
\end{gathered}
$$

Portanto, aplicando-se o método de Lanczos na Eq. (2.68) obtém-se $\lambda$ e $\{\mathbf{V}\}$, e utilizando a Eq. (2.69) recupera-se os autovetores $\{\mathbf{W}\}$.

Outro método para resolver problemas de autovalor e autovetor piezelétricos é aplicar condensação estática ao sistema da Eq. (2.59) para eliminar os GDLs de potencial 
elétrico internos (uma vez que as cargas elétricas nodais internas são zero), mantendo na formulação somente os GDLs de potencial elétrico dos eletrodos, como descrito em Naillon, Coursant e Besnier (1983). A vantagem deste método é que as matrizes obtidas são positiva-definidas, e então, o problema de autovalor pode ser resolvido aplicando solucionadores comuns. Entretanto, a banda da matriz de rigidez total é destruída e por isso, deve-se lidar com a matriz inteira o que aumenta a necessidade de memória, se tornando inviável se o modelo de MEF for grande.

Neste trabalho, considerando que o método de otimização necessita de uma grande quantidade de memória, e em otimização topológica os modelos do MEF são geralmente grandes para se obter uma imagem com alta definição da topologia final, foi elaborada uma rotina, baseada no esquema de armazenagem de matrizes de Yong (1995), Yong e Cho (1994), utilizando uma função do MatLab ("eigs"). Esta função utiliza o pacote ARPACK, que se baseia numa variante do método de Arnoldi, chamada de "Método de Arnoldi Reiniciada Implicitamente", que na literatura é denominada "Implicity Restarted Arnoldi Method" (IRAM), para calcular alguns autovalores e autovetores. Quando as matrizes são simétricas (que é o caso das matrizes obtidas neste trabalho), o método de reduz numa variante do método de Lanczos (LEHOUCQ; SORENSEN; YANG, 1998), o qual é o mais utilizado para problemas de autovalor e autovetor piezelétricos, sendo robusto e confiável o suficiente para a obtenção de uma solução precisa. A rotina utiliza matrizes esparsas (somente aloca na memória os valores diferentes de zero), preservando a banda da matriz de rigidez total.

Como a rotina "eigs" executa internamente a fatoração Cholesky para decompor a matriz de massa, e então fazer o cálculo dos autovalores e autovetores, não é possível entrar com a matriz de massa $[\overline{\mathbf{M}}]$, já que ela é positiva semi-definida. Para esses casos, a rotina "eigs" possibilita que o usuário forneça uma variável contendo a informação de que a fatoração Cholesky já foi executada.

Então, antes de executar a rotina "eigs", é necessário fatorar a matriz de massa assim como nas Eqs. (2.64) e (2.65), e dar como entrada da rotina, as matrizes $[\overline{\mathbf{K}}]$ e $\left[\overline{\mathbf{M}}_{\mu}\right]$, ao invés de $[\overline{\mathbf{M}}]$, sendo que:

$$
\left[\overline{\mathbf{M}}_{\mu}\right]=\left[\begin{array}{cc}
\mathbf{L}_{\mu} & 0 \\
0 & 0
\end{array}\right]
$$

As outras entradas da rotina "eigs" são: o número de autovalores desejados, o deslocamento da freqüência (que pode ser um escalar ou pode ser uma string informando 
se deseja os menores ou maiores valores), e uma variável option, na qual deve ser incluída a informação de que a matriz $\left[\overline{\mathbf{M}}_{\mu}\right]$ já é a decomposição Cholesky da matriz $[\overline{\mathbf{M}}]$.

\subsection{Busca do Modo de Vibrar Baseado no Método MAC}

Para problemas de OT dinâmicos, um dos problemas relacionados com o cálculo de autovalores e autovetores é o salto de modos. Este fenômeno ocorre quando, decorrente das alterações nas propriedades do modelo de elementos finitos durante a OT, o modo $n$ passa a ser o modo $n+1$, e vice-e-versa. Assim, se a otimização estiver sendo feita para o modo $n$, que é um modo de pistão, por exemplo, onde os deslocamentos da face superior devem estar em fase (Fig. 2.7(a)), e caso haja o salto de modos, a otimização continuará no modo $n$ (Fig. 2.7(c)), porém, este não será o modo de pistão, e sim o modo $n+1$ (Fig. 2.7(d)). A Fig. 2.7 representa este exemplo.

Então, para este exemplo, o que deseja é continuar com a otimização no modo $n+1$. Contudo, em alguns casos, o modo $n$ pode passar a ser o modo $n+2$, ou $n-1$, ou qualquer outro modo, porém próximo do original. Para isso, uma formulação deve ser incluída a cada iteração para procurar o modo desejado entre os que foram calculados.

Neste trabalho, foi proposta uma formulação para a busca de modos que utiliza o método do "Modal Assurance Criterion" (MAC) (traduzindo literalmente fica "Critério de Garantia Modal"), cuja definição é (EWINS, 1986; KIM; KIM, 2000):

$$
\operatorname{MAC}\left(\boldsymbol{\Phi}_{a}, \boldsymbol{\Phi}_{b}\right)=\frac{\left|\boldsymbol{\Phi}_{a}^{T} \boldsymbol{\Phi}_{b}\right|^{2}}{\left(\boldsymbol{\Phi}_{a}^{T} \boldsymbol{\Phi}_{a}\right)\left(\boldsymbol{\Phi}_{b}^{T} \boldsymbol{\Phi}_{b}\right)}
$$

onde $\boldsymbol{\Phi}_{a}$ e $\boldsymbol{\Phi}_{b}$ são os dois autovetores que estão sendo comparados. O valor de MAC varia entre 0 e 1. Quando o valor de MAC for igual a 1, então os dois autovetores $\boldsymbol{\Phi}_{a}$ e $\boldsymbol{\Phi}_{b}$ representam o mesmo modo de vibrar (KIM; KIM, 2000).

Esta formulação permite que se faça a comparação entre $\boldsymbol{\Phi}_{a}$ e $\boldsymbol{\Phi}_{b}$ em apenas alguns pontos, o que é interessante pois estamos interessados em otimizar o modo de pistão, que foi definido como aquele que possui as amplitudes dos pontos da face superior do sonotrodo em fase. Então $\boldsymbol{\Phi}_{a}$ e $\boldsymbol{\Phi}_{b}$ são dois vetores contendo os valores dos deslocamentos do modo de pistão apenas dos pontos dessa face do sonotrodo. Isso já é condição suficiente para que, considerando que $\boldsymbol{\Phi}_{b}$ é o modo de pistão que servirá para comparação, o modo $\boldsymbol{\Phi}_{a}$ procurado tenha todos as amplitudes em fase. Contudo, pode-se inserir uma restrição para garantir ainda mais que todas as amplitudes de $\boldsymbol{\Phi}_{a}$ estejam em fase. Definindo que 


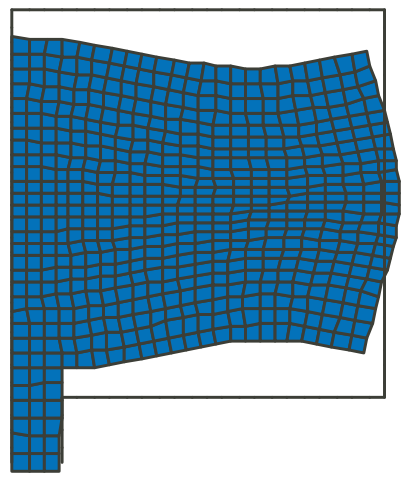

(a) Modo $n$ de pistão $(22094 H z)$

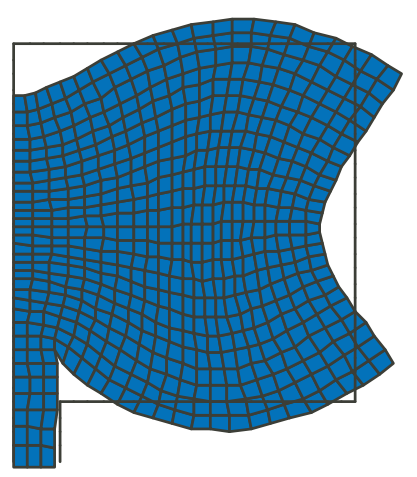

(c) Modo $n(22094 H z)$

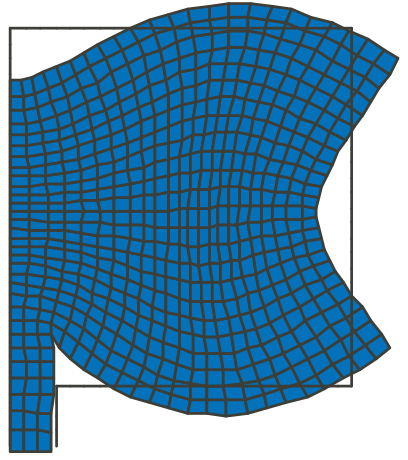

(b) Modo $n+1$ $(23419 H z)$

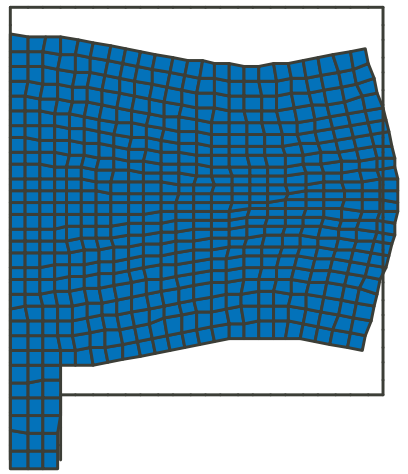

(d) Modo $n+1$ de pistão $(23419 H z)$

Figura 2.7: Representação de inversão de modos: antes da inversão (a) e (b), e depois da inversão (c) e (d).

$N$ é o número de pontos na face superior do sonotrodo, $\boldsymbol{\Phi}_{a}$ será o modo de pistão se a soma dos sinais de seus termos forem iguais a $N$ ou $-N$.

Portanto, utilizando o MAC somente nos pontos que são de interesse na otimização, e utilizando a restrição descrita, pode-se garantir que o modo $\Phi_{a}$ é o modo de pistão que estamos interessados em otimizar. 


\section{MÉTODO DE OTIMIZAÇÃO TOPOLÓGICA (MOT)}

\subsection{Introdução}

O Método de Otimização Topológica (MOT) consiste num método computacional que permite, segundo um certo critério de custo, projetar a topologia ótima de uma estrutura. Basicamente, o Método de Otimização Topológica distribui o material no interior de um domínio fixo de forma a maximizar (ou minimizar) uma função custo especificada, por exemplo, máxima rigidez e mínimo volume de material. O material em cada ponto do domínio pode variar de ar (não há presença de material) até sólido (total presença de material), podendo também assumir densidades intermediárias entre ar e sólido, de acordo com um modelo de material definido.

Um algoritmo de otimização é utilizado para encontrar a distribuição ótima de material, o que, de forma iterativa, torna o processo rápido; caso contrário seriam necessárias muitas análises para encontrar a distribuição ótima do material no domínio. Para acelerar o processo de busca da distribuição ótima de material, os métodos de otimização utilizam-se da informação dos gradientes (ou derivadas) da função custo em relação à quantidade de material em cada elemento. A distribuição de material é representada, por exemplo, associando um valor (variável de projeto) a cada elemento (subdomínio), obtido da discretização do domínio inicial.

Dessa forma, a OT combina essencialmente métodos de otimização com um método numérico de análise, por exemplo, o método de elementos finitos (MEF). Outros métodos numéricos de análise podem ser utilizados; no entanto, devem ser genéricos o suficiente de forma a lidar com estruturas de formas complexas resultantes da otimização.

O MOT é um assunto recente no campo da otimização estrutural. Contudo, os conceitos básicos que dão suporte teórico ao método foram estabelecidos a mais de um século (BENDSøE; SIGMUND, 2003). Para entender melhor como surgiu o método e como ele 
se estabeleceu atualmente nos projetos de estruturas mecânicas, devemos antes conhecer as diferentes formas de otimização estrutural existentes.

Essencialmente existem três abordagens em otimização: otimização paramétrica, otimização de forma e otimização topológica. Na otimização paramétrica, as variáveis de projeto são as dimensões ou as razões de dimensões da peça. A otimização de forma permite alterar a forma da estrutura de maneira a encontrar a forma ótima. Neste tipo de otimização devido às formas complexas que podem ser obtidas é utilizado em geral o Método de Elementos Finitos (MEF) para a análise das estruturas durante a otimização. A principal desvantagem da otimização de forma nesse caso é que com a alteração da forma da estrutura a malha de MEF é distorcida exigindo um remalhamento do domínio durante a otimização, o que em geral produz resultados pouco adequados. Por último, a Otimização Topológica permite determinar a presença de novos furos na topologia da estrutura, permitindo obter altas reduções de massa e um grande aumento de desempenho, além disso, elimina o problema de remalhamento da malha de elementos finitos como acontece na otimização de forma. Para ilustrar estas técnicas, a Fig. 6.1 mostra os resultados obtidos para a otimização de um domínio onde deseja-se máxima rigidez da estrutura com relação ao carregamento aplicado.

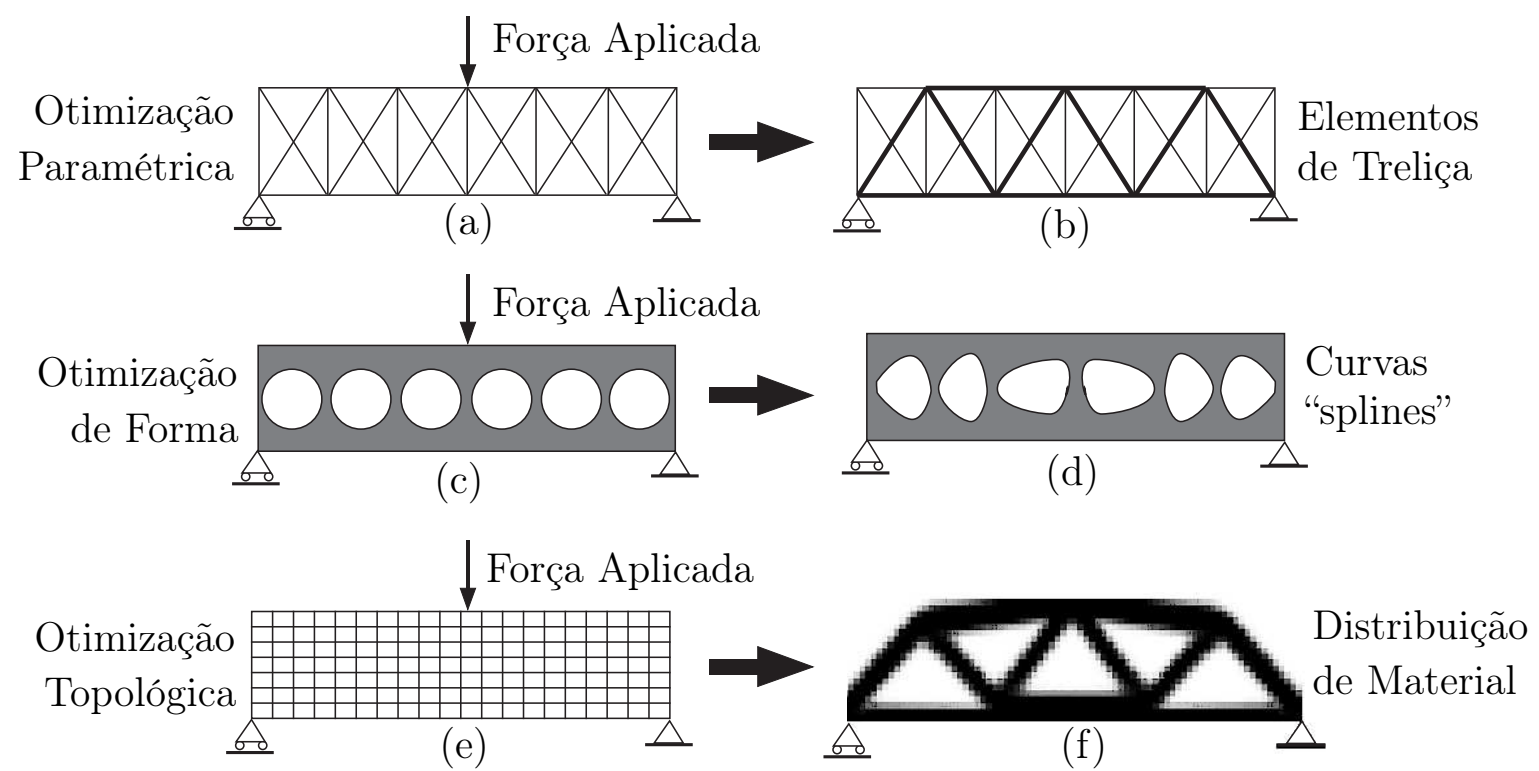

Figura 3.1: Solução de um problema por diferentes abordagens de otimização.

A otimização topológica é a mais genérica, sendo que a quantidade de material removida (para a mesma função custo) é maior do que nos outros métodos de otimização, conseqüentemente, obtêm-se a estrutura mais leve com melhor desempenho. 


\subsection{Histórico}

No final da década de 80, Bendsøe e Kikichi (1988) introduziram uma metodologia alternativa para a otimização de forma, que parte do princípio de fixar o domínio inicial da estrutura, e portanto, manter inalterado a malha e elementos finitos utilizado no processo de otimização. Assim, surge a metodologia para OT de estruturas mecânicas, baseado no conceito de domínio fixo estendido de projeto, e inicialmente, no método da homogeneização (GUEDES; KIKUCHI, 1990) como modelo de material.

Essa metodologia foi fortemente inspirada nas conclusões obtidas nos trabalhos que lidam com otimização da distribuição de espessuras em placas e chapas (CHENG; OLHOFF, 1981, 1982) e otimização para projetos de barras de torção construídas com dois materiais em diferentes proporções volumétricas (LURIE; CHERKAEV; FEDOROV, 1982b, 1982a; STRANG; KOHN, 1986). Cheng e Olhoff (1981) investigaram a formulação matemática para o problema de maximização de rigidez (com restrição de volume) de placas delgadas, onde a variável de projeto é a espessura da placa, e concluíram que para este problema de otimização existem várias soluções ótimas locais. Conclusão também obtida por Rozvany et al. (1982), ou seja, os resultados obtidos indicam que a solução ótima é uma placa que ao longo da espessura é composta por regiões com infinitos reforços infinitesimais (nervuras), cujo comportamento é similar a um material composto por infinitas micro-estruturas, que por sua vez pode ser representada por um modelo de material.

Os modelos de material constituem a base da OT e podem ser divididos em duas categorias, método da homogeneização e método das densidades. Uma revisão sobre os modelos de material utilizados no MOT pode ser vista em Hassani e Hinton (1998b). Em termos matemáticos a introdução de uma micro-estrutura na formulação de um problema estrutural permite a relaxação do variacional do problema de otimização. O cálculo da relaxação dos funcionais na otimização estrutural estão detalhados na literatura (STRANG; KOHN, 1986; KOHN; STRANG, 1986a, 1986b, 1986c). Bendsøe (1989) descreve várias maneiras de se conseguir a relaxação do variacional através da introdução de um modelo de material baseado na distribuição de densidades na micro-estrutura, dentre eles o chamado método de densidades.

A metodologia para OT foi definida por Bendsøe e Kikichi (1988) e Suzuki e Kikuchi (1991), que implementaram o método para resolver com sucesso vários exemplos de otimização estrutural, cuja função objetivo do problema é a maximização de rigidez sujeita à restrição de volume do material. Na seqüência, Diaz e Bendsøe (1992) apresentaram uma formulação para o problema de maximização de rigidez de estruturas elásticas submetida 
a várias cargas não simultâneas. Thomsen (1992) trataram numericamente uma extensão do método aplicada a otimização de estruturas compostas por mais de um material. Outra extensão do método para aplicação em projeto de reforçamento de chapas de carrocerias de automóveis pode ser encontrada em Fukushima, Suzuki e Kikuchi (1993). Problemas de OT considerando freqüência de ressonância em estruturas contínuas são descritos em Diaz e Bendsøe (1992) e Ma e Kikuchi (1995). A análise transiente em estruturas como problema de OT foi realizada por Min et al. (1999) e Turteltaub (2001).

Uma revisão bibliográfica ampla pode ser encontrada na referência Bendsøe e Sigmund (2003)

\subsection{Procedimento para Implementação da Otimização Topológica}

O procedimento típico de projeto estrutural utilizando a otimização topológica é apresentado na Fig. 3.2. É importante observar que no processo de solução do problema de otimização o domínio de projeto é discretizado, e por isso torna-se necessária à utilização de métodos numéricos.

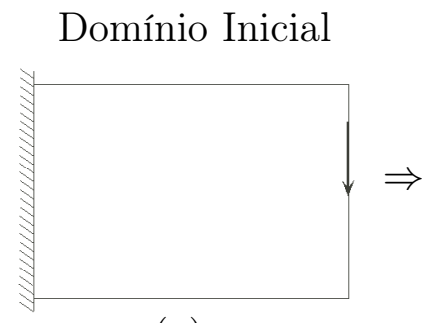

(a)

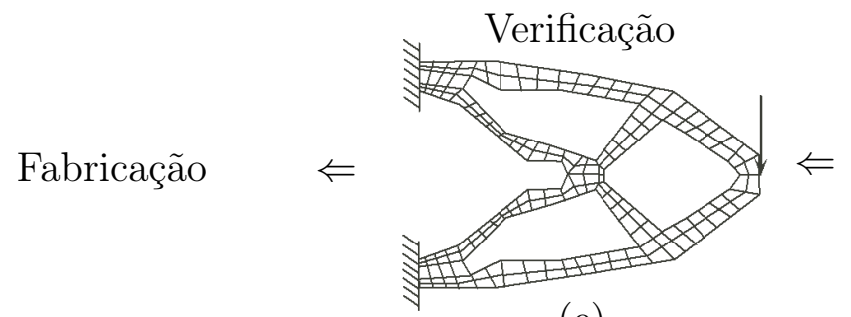

(e)
Topologia Obtida

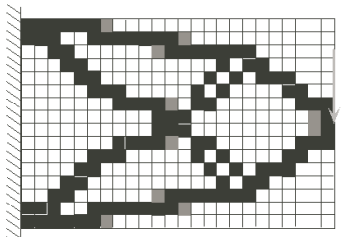

(c)

$\Downarrow$

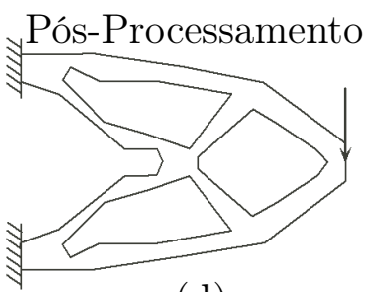

(d)

Figura 3.2: Procedimento típico de projeto estrutural por otimização topológica.

O primeiro passo consiste em definir o domínio $(\Omega)$ do projeto no qual a estrutura pode existir. Esse domínio é limitado pelos pontos de aplicação de carga e pelas condições de contorno da estrutura, ou seja, pontos onde os deslocamentos são restringidos. É importante nessa fase definir o domínio $(\Omega)$ com o maior tamanho possível, de forma 
a não limitar o domínio de trabalho da Otimização Topológica, pois como em qualquer método de otimização, quanto mais restrições são impostas, menor o espaço de soluções para o problema. No segundo passo o domínio $(\Omega)$ é discretizado em elementos finitos e são aplicadas as condições de contorno.

Na terceira etapa, os dados do domínio $(\Omega)$ são fornecidos ao software de otimização topológica, que num processo iterativo, distribui o material no domínio $(\Omega)$ de forma a minimizar (ou maximizar) a função objetivo especificada. O resultado obtido é mostrado na Fig. 3.2(c), onde a cor escura indica à presença de material e a cor branca a ausência de material no ponto do domínio $(\Omega)$. Note que podem surgir pontos com cores intermediárias, denominados de escalas de cinza (ou "gray scale"), que indicam presença de materiais intermediários, os quais são difíceis de serem implementados na prática, no entanto são inerentes ao problema de Otimização Topológica.

Outro problema é a "instabilidade de tabuleiro" onde elementos de cor escura e elementos de cor branca aparecem dispostos em forma de tabuleiro de xadrez. As características da "instabilidade de tabuleiro" podem ser observadas mais adiante. Portanto, a "escala de cinza" e a "instabilidade de tabuleiro" são indesejáveis e prejudicam o pós-processamento dos resultados da otimização (quarta etapa).

Esse pós-processamento, que pode ser feito utilizando métodos de processamento de imagem ou simplesmente desenhando-se uma estrutura baseada na imagem obtida pela OT, consiste na remoção das eventuais "escalas de cinza" e a suavização do contorno da topologia obtida, ajustando-a de forma a ser fabricada. O quinto passo consiste em se verificar o resultado final da estrutura.

Em geral, os resultados gerados por OT não são intuitivos e é interessante fazer uma verificação da estrutura final utilizando o MEF, para criarmos confiança na solução através da comprovação da optimalidade do resultado. Finalmente, a última etapa é a fabricação da estrutura, onde, atualmente, existem várias técnicas de fabricação que permitem obter estruturas com formas complexas, como, a prototipagem rápida, a eletro-erosão a fio, processo de corrosão química pelo método de litografia, entre outras.

\subsection{Conceitos Teóricos da Otimização Topológica}

A OT baseia-se em dois conceitos teóricos principais, domínio fixo estendido e modelo de material, que serão apresentados a seguir. 


\subsubsection{Domínio Fixo Estendido}

O domínio estendido fixo de projeto $(\Omega)$ é o espaço no qual o algoritmo de otimização topológica pode sintetizar a estrutura.

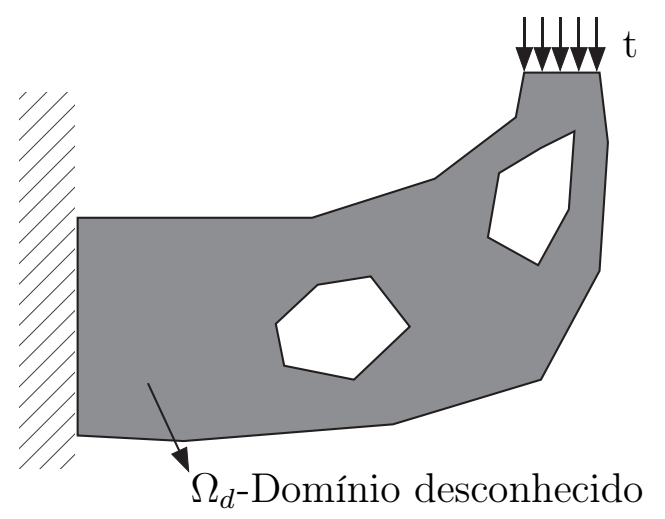

(a)

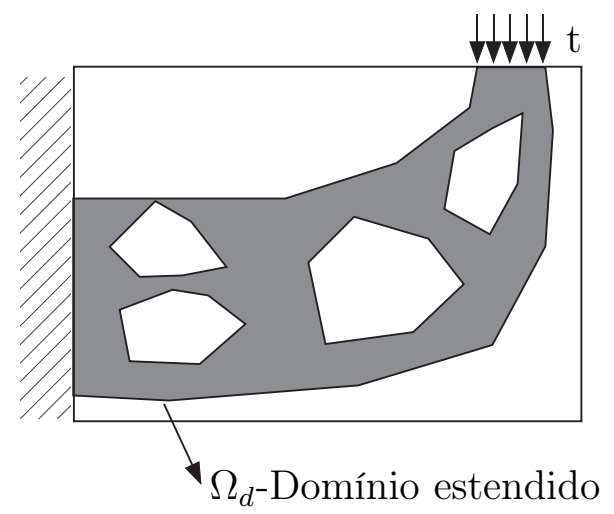

(b)

Figura 3.3: Representação de um domínio desconhecido (a) e do domínio estendido fixo(b).

Como pode ser visto na Fig. 3.3(b), o domínio estendido fixo consiste num domínio de forma fixa limitado pelos pontos de apoio da estrutura e pontos de aplicação de carregamento, que conterá a estrutura desconhecida. Ou seja, num problema de OT, sem quaisquer informações sobre a geometria final da estrutura, a forma ótima é obtida sendo influenciada pelas restrições de deslocamentos (pontos de fixação), pela quantidade de material utilizada e pela aplicação de cargas. O objetivo da otimização topológica é determinar os espaços sem material ou "vazio" (variável de projeto) e a conectividade da estrutura através da remoção e adição de material nesse domínio. O problema de otimização consiste, portanto, em se encontrar a distribuição ótima de propriedades de materiais no domínio estendido fixo. A grande vantagem em relação a otimização de forma é que o modelo de elementos finitos do domínio não é alterado durante o processo de otimização, sendo alterado somente a distribuição de material nos elementos, pois sendo o domínio fixo, as derivadas de uma função $q$ são calculadas utilizando a seguinte expressão:

$$
\frac{\partial}{\partial A_{n}} \int_{\Omega} q d \Omega=\int_{\Omega} \frac{\partial q}{\partial A_{n}} d \Omega
$$

onde $A_{n}$ é uma variável de projeto, e $q$ é uma função contínua e derivável definida no domínio. 


\subsubsection{Modelo de Material}

O modelo de material é uma equação que define a mistura em micro-escala de dois ou mais materiais (um deles pode ser "vazio") permitindo passar da condição de zero material ("buraco") a sólido em cada ponto do domínio.

Para materiais isotrópicos, pode-se escrever:

$$
C(\mathbf{x})=\chi(\mathbf{x}) C_{0}
$$

onde $C_{0}$ é a propriedade básica do material a ser distribuído e $\chi(\mathbf{x})$ é uma função discreta que caracteriza a estrutura a ser otimizada, e é definida em cada ponto $\mathbf{x}$ do domínio $\Omega$ da seguinte maneira:

$$
\chi(\mathbf{x})=\left\{\begin{array}{lll}
1 & \text { se } & \mathbf{x} \in \Omega_{D} \\
0 & \text { se } & \mathbf{x} \in \Omega \backslash \Omega_{D}
\end{array}\right.
$$

sendo $\Omega_{D}$ a região onde há presença de material, inserida num domínio $\Omega$, representado na Fig. 3.4.

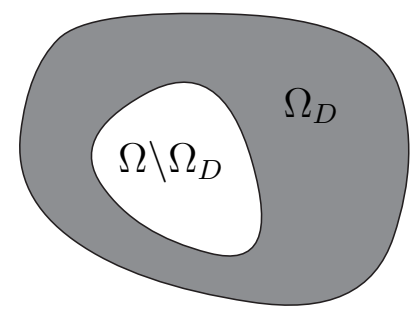

Figura 3.4: Definição do domínio de projeto.

Contudo, o problema discreto pode não possuir solução, sendo necessária uma relaxação para o problema contínuo. Essa relaxação das variáveis de projeto consiste em permitir que elas assumam valores intermediários entre 0 e 1. A princípio, os estágios intermediários de materiais não têm um significado físico, sendo apenas decorrentes de um recurso matemático para relaxação do problema. Existem vários modelos de material que podem ser utilizados, entre eles o método das densidades apresentado no item a seguir.

\subsection{Método das Densidades}

O método de densidades consiste em uma equação matemática que define o valor da densidade em cada ponto do domínio $(\Omega)$ em função da propriedade efetiva do material base utilizado no projeto, essencialmente simulando uma microestrutura. 
Comumente utiliza-se a formulação SIMP ("Simple Isotropic Material with Penalization”) (BENDSøE; SIGMUND, 1999, 2003), cuja equação é dada por:

$$
C(\mathbf{x})=\gamma(\mathbf{x}) C_{0}
$$

onde $\gamma(\mathbf{x})$ é interpretada como uma função de distribuição contínua da variável de projeto (pseudo-densidade), $0<\gamma_{\min } \leq \gamma(\mathbf{x}) \leq 1$ e $\mathbf{x} \in \Omega$. O valor $\gamma_{\min }$ é inserido para evitar instabilidades numéricas quando for necessário o cálculo da matriz inversa. $C(\mathbf{x})$ é o valor da propriedade do material em cada elemento do domínio, $C_{0}$ é a propriedade do material base isotrópico, sendo que essas propriedades podem ser o módulo de elasticidade $\left(E_{0}\right)$ ou a densidade $\left(\rho_{0}\right)$. Portanto, podemos dizer que a pseudo-densidade, em cada ponto do domínio $(\Omega)$ pode variar de "zero" (não há presença de material) à "um" (total presença de material), ou seja, teremos valores intermediários de materiais. Entretanto, apesar de ter estabelecido a relaxação do problema e permitir obter um espaço de solução fechado, um outro problema é gerado, a "escala de cinza", que do ponto de vista prático, é difícil de ser pós-processado.

Para evitar o excesso de ocorrências de pseudo-densidades intermediárias, penalizam-se os valores intermediários de $\rho(\mathbf{x})$ da seguinte maneira, já substituindo a propriedade $C$ pelo módulo de elasticidade e pela densidade do material:

$$
E(\mathbf{x})=\gamma(\mathbf{x})^{p} E_{0} \quad, \quad \rho(\mathbf{x})=\gamma(\mathbf{x})^{q} \rho_{0}
$$

onde $p$ e $q$ são os fatores que penalizam as pseudo-densidades, fazendo com que os valores intermediários se aproximem de 0 e 1, e portanto, diminuindo as "escalas de cinza".

Contudo, quando considerada uma situação dinâmica de estruturas onde há necessidade de se calcular autovalores, o modelo SIMP acarreta modos localizados em regiões onde a pseudo-densidade possui um valor baixo (NEVES; RODRIGUES; GUEDES, 1995). Modos localizados ocorrem em áreas em que a variável de projeto tem o valor mínimo admissível ou perto desse valor. Estas áreas são mais flexíveis comparadas com as áreas em que a variável apresenta valores altos, e conseqüentemente controlarão os autovalores e autovetores mais baixos de toda a estrutura (PEDERSEN, 2000). A figura a seguir representa os modos localizados de uma placa, onde a região em branco representa o valor da pseudo-densidade baixa, e a região em preto representa valor máximo para a pseudo-densidade.

Isso acontece pelo fato de que, ao se utilizar o modelo SIMP para o módulo de elasticidade e para a densidade (Eq. (3.5)), o módulo de elasticidade converge para zero 


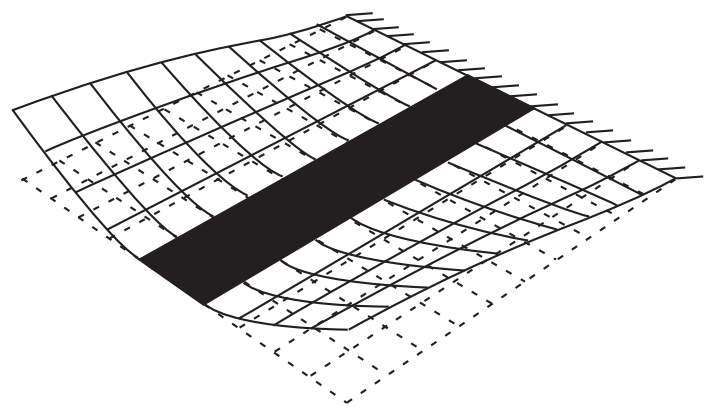

Figura 3.5: Representação dos modos localizados de uma placa em vibração.

mais rápido que a densidade quando o valor da variável se aproxima do seu valor mínimo, pois geralmente $p$ (penalização do módulo de elasticidade) é maior que $q$ (penalização da densidade) (valores típicos para os expoentes são $p=3$ e $q=1$ ).

Para se estudar a influência dos diferentes valores das penalidades nos modos localizados define-se a razão massa-rigidez $\left(\kappa_{1}\right)$ proveniente do cálculo de autovalores e autovetores das Eqs. (2.67) e (2.68), da seguinte maneira:

$$
\kappa_{1}=\frac{\rho(\mathbf{x})}{E(\mathbf{x})}=\frac{\gamma(\mathbf{x})^{q} \rho_{0}}{\gamma(\mathbf{x})^{p} E_{0}}=\gamma(\mathbf{x})^{q-p} \frac{\rho_{0}}{E_{0}}
$$

Como $p$ é maior que $q$, o valor de $\kappa_{1}$ tende ao infinito quando a variável de projeto tende a seu valor mínimo, como pode ser visto no gráfico da Eq. (3.6).

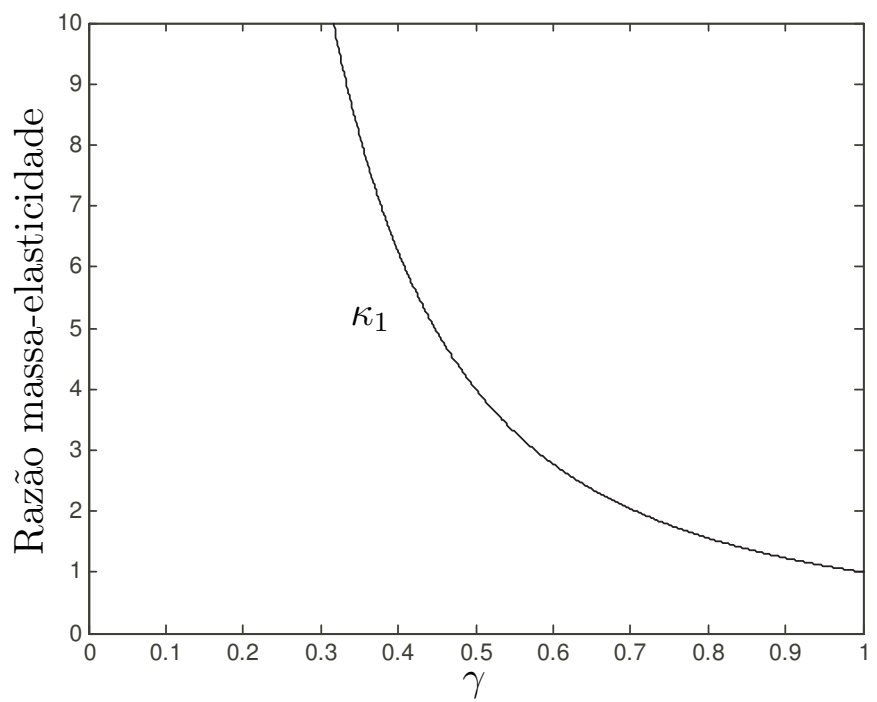

Figura 3.6: Curva de interpolação da razão massa-rigidez para o modelo SIMP aplicado na densidade e no módulo de elasticidade.

Para contornar esse problema, Stolpe e Svanberg (2001), Bendsøe e Sigmund (2003) e Hansen (2005) propõe um modelo alternativo, onde o módulo de elasticidade $E$ é 
interpolado como:

$$
E(\mathbf{x})=E_{\min }+\frac{\gamma(\mathbf{x})}{1+p(1-\gamma(\mathbf{x}))}\left(E_{0}-E_{\min }\right)
$$

onde $E_{0}$ é o valor do módulo de elasticidade para o material base e $E_{\text {min }}$ é o valor mínimo para tal propriedade. Geralmente utiliza-se (BENDSøE; SIGMUND, 2003):

$$
E_{\min }=10^{-6} E_{0}
$$

Essa formulação é chamada de RAMP ("Rational Approximation of Material Properties"), e com ela, os problemas em áreas de baixa densidade são evitados, pois agora, a razão massa-rigidez fica $\left(\operatorname{com} E_{\min }=0\right)$ :

$$
\kappa_{2}=\frac{\rho(\mathbf{x})}{E(\mathbf{x})}=\frac{\gamma(\mathrm{x})^{q} \rho_{0}}{\frac{\gamma(\mathbf{x})}{1+p(1-\gamma(\mathbf{x}))} E_{0}}=\gamma(\mathbf{x})^{q-1}[1+p(1-\gamma(\mathbf{x}))] \frac{\rho_{0}}{E_{0}}
$$

e, por ser uma função côncava, qualquer valor de $p$ e $q$ pode ser utilizado que $\kappa_{2}$ nunca tenderá ao infinito. A curva do modelo RAMP pode ser vista na Fig. 3.7, para $p=3$ e $q=1$, junto com a curva do modelo SIMP para comparação.

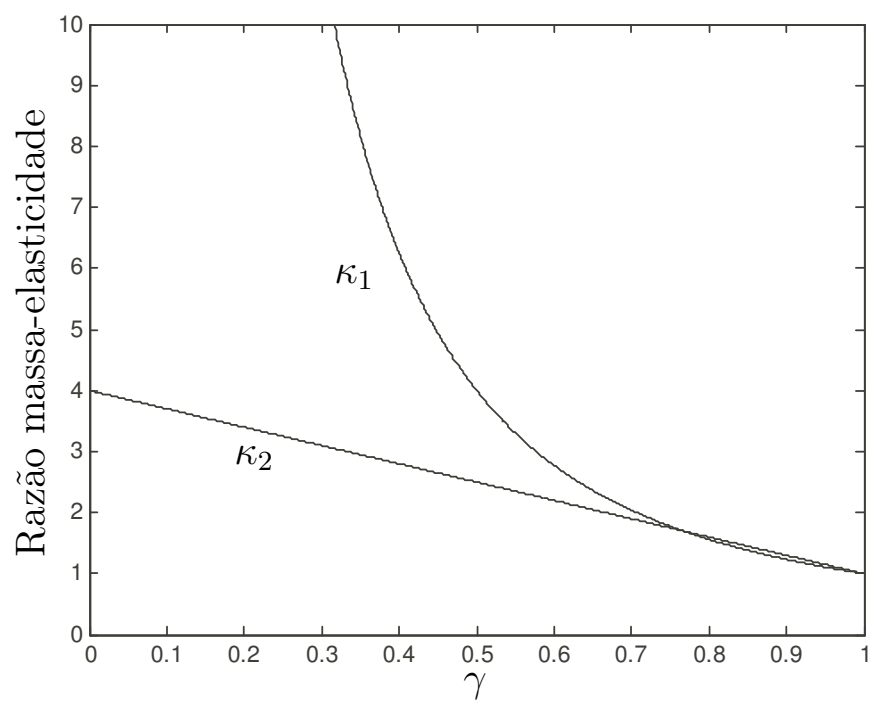

Figura 3.7: Curvas de interpolação da razão massa-rigidez para os modelos RAMP e SIMP.

A utilização do modelo RAMP no módulo de elasticidade e do modelo SIMP na densidade é validada mediante avaliação detalhada dos gráficos da Fig. 3.8, onde está mostrado a comparação entre outras combinações da utilização desses modelos, e diferentes valores para as penalizações $p$ e $q$.

Além de $\kappa_{1}$ (SIMP na densidade e na rigidez) e de $\kappa_{2}$ (SIMP na densidade e RAMP na rigidez), foram avaliados também, o caso onde é aplicado o modelo RAMP na densidade 
e na rigidez $\left(\kappa_{3}\right)$ e o caso do RAMP na densidade e do SIMP na rigidez $\left(\kappa_{4}\right)$, que são definidos nas equações a seguir:

$$
\kappa_{3}=\frac{1+p(1-\gamma)}{1+q(1-\gamma)} ; \quad \kappa_{4}=\frac{\gamma^{1-p}}{1+q(1-\gamma)}
$$

Como pode ser visto no gráfico da Fig. 3.8(a), ao serem utilizadas as penalizações $p=1$ e $q=1$ não é verificada qualquer instabilidade numérica. Porém, como é utilizado o método de continuação, aumentando-se a ordem das penalizações, $\operatorname{com} p>q$, as curvas $\kappa_{1}$ e $\kappa_{4}$ sempre tendem ao infinito, como pode ser visto nos gráficos das Figs. 3.8(b), 3.8(c) e $3.8(\mathrm{~d})$.

Nesses mesmos gráficos, as curvas $\kappa_{2}$ e $\kappa_{3}$ possuem uma estabilidade numérica, mas a vantagem de se utilizar $\kappa_{2}$ é que, quanto mais alto os valores das penalizações, a curva converge para zero quando a variável de projeto possui valores pequenos. Ou seja, para elementos cuja pseudo-densidade é baixa, a razão massa-rigidez tende a zero, minimizando assim os modos localizados dessas regiões.

Uma outra opção é utilizar $p<q$, como pode ser visto no gráfico da Fig. 3.8(e), onde instabilidades numéricas aparecem somente na curva $\kappa_{3}$. Ou seja, pode-se utilizar o modelo SIMP tanto na rigidez quanto na densidade (função $\kappa_{1}$ ) pois sua curva possui comportamento semelhante à curva da função $\kappa_{2}$. Porém, em se tratando de um processo iterativo, onde a distribuição de pseudo-densidades intermediárias é importante para a convergência de uma solução ótima global, ou até mesmo uma solução ótima local, valores muito altos para $q$ (por exemplo $q=6$ ) não são utilizadas (PEDERSEN, 2000). Isso ocorre pois apenas os valores das pseudo-densidades próximas do material sólido serão levadas em conta na análise modal. No entanto, utilizando o método da continuação essa opção é válida, pois valores altos para as penalizações somente irão atuar quando a solução já estiver perto de uma solução ótima global ou de uma solução ótima local bem definida.

Portanto, o método de densidades é um modelo de material que, comparado a outros métodos, é menos complexo de ser implementado numericamente num algoritmo de otimização, pois possui apenas uma variável $(\gamma)$. Além disso, o método das densidades também permite obter topologias com uma quantidade mínima de "escalas de cinza". Contudo, essas topologias obtidas são soluções ótimas locais e por isso devemos ter uma relação de compromisso, ou seja, obter uma solução ótima local bem definida, o mais próxima possível da solução ótima global e que atenda aos requisitos de viabilidade de construção para a estrutura projetada. 


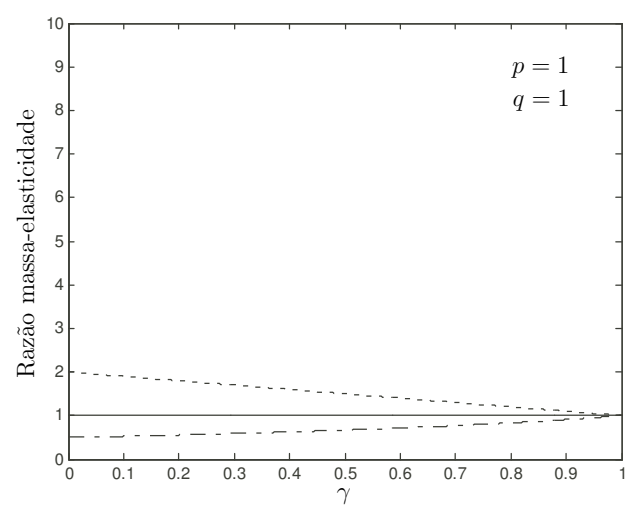

(a)

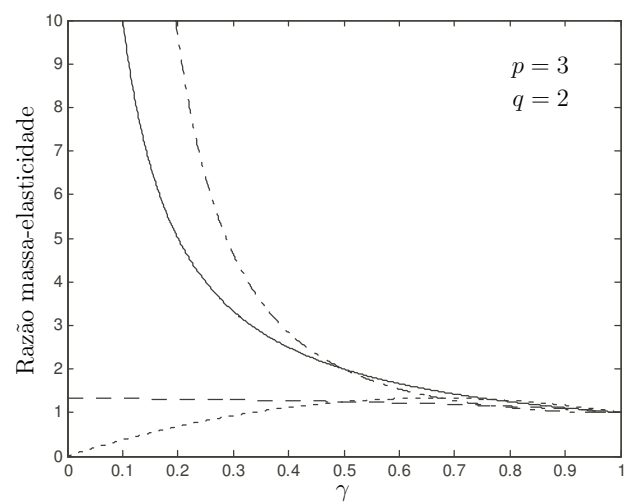

(c)

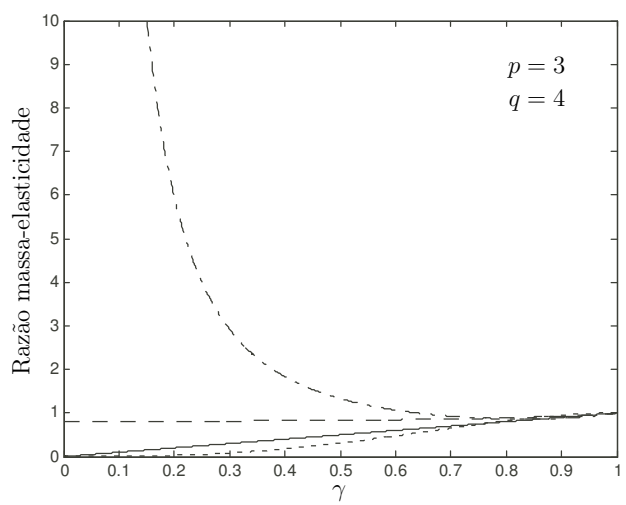

(e)

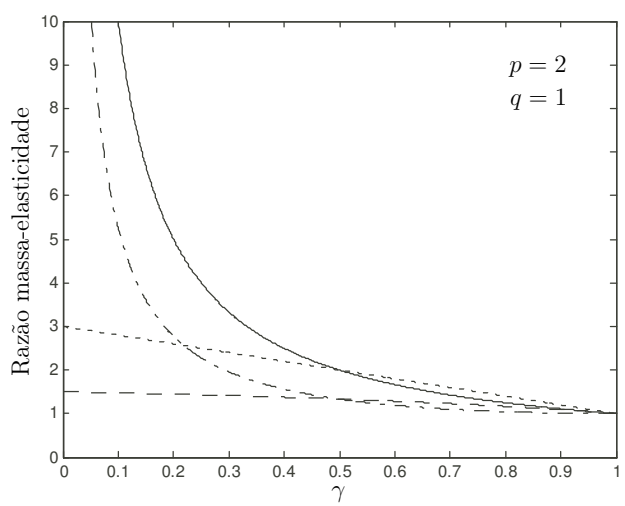

(b)

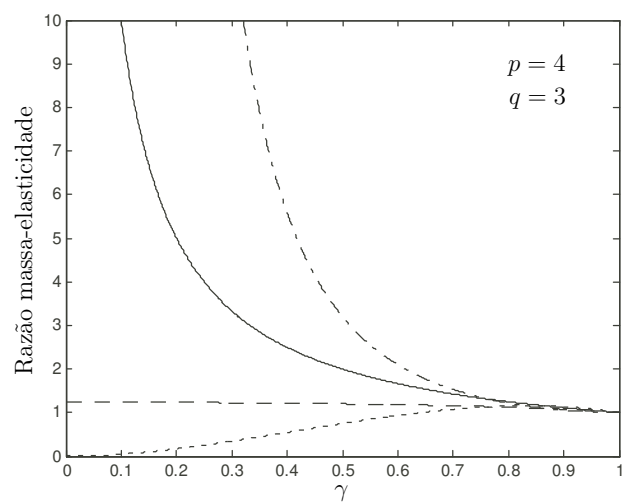

(d)

Figura 3.8: Gráficos comparativos entre a razão massa-rigidez para os vários casos de combinação entre os modelos SIMP e RAMP.

\subsection{Aspectos Numéricos da OT}

Neste item são apresentados alguns aspectos numéricos importantes da otimização topológica que, se não forem contornados, comprometem a solução final. 


\subsubsection{Escala de Cinza}

A presença de materiais intermediários (ou "escala de cinza" como é chamado na literatura) ocorre quando um problema é relaxado, definindo-se um modelo de material, na tentativa de se contornar a não existência da solução do problema discreto $(0-1)$ de OT. A relaxação garante a existência da solução ótima, no entanto, por apresentar materiais intermediários, tornará difícil a sua fabricação. Uma representação esquemática da escala de cinza pode ser vista na Fig. 3.9.

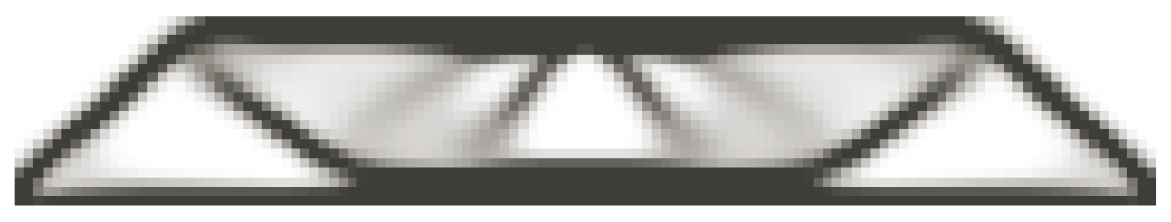

Figura 3.9: Representação da escala de cinza.

Com o intuito de obter uma solução que possa ser fabricada procura-se recuperar o caráter discreto da distribuição de material, utilizando-se por exemplo, o fator de penalização $p$ ou $q$ (para rigidez e densidade respectivamente) no modelo de material. Contudo, ao utilizar valores muito altos para as penalizações desde o início do processo iterativo, retorna-se para os problemas da formulação discreta.

Em geral, os métodos de penalização devem vir acompanhados da utilização do método de continuação, no qual o valor da penalização é modificado do seu valor inicial até seu valor limite. O método de continuação é utilizado pois, geralmente, os métodos de penalização tendem a aumentar o grau da função objetivo, de modo que esta passa a ter um maior número de mínimos locais. A continuação das penalidades é feita ao longo do processo iterativo. Normalmente começa-se com o valor, por exemplo, $p=1$ e, após convergir para uma solução, o valor de $p$ é aumentado, para então convergir a uma segunda solução. Este procedimento é repetido até que $p$ atinja seu valor limite, que, em geral, vale $p=3$ ou $p=4$ (Bendsøe, 2003). O método de continuação é necessário para que a solução MOT não convirja para um mínimo local indesejável, pois para valores de $p=3$ ou $p=4$, a função objetivo, em geral, será mal comportada uma vez que se aproxima de uma função discreta.

Uma representação esquemática dessa situação é exposta na Fig. 3.10, onde se verifica que a função $f(\gamma)$ é convexa, enquanto a função $f\left(\gamma^{3}\right)$ é mal comportada pois possui vários ótimos locais. Assim, iniciando com $p=1$ é possível que o método e otimização convirja para o ótimo global, e posteriormente, com o aumento de $p$, a solução convirja para um ótimo local próximo ao ótimo global do problema. 


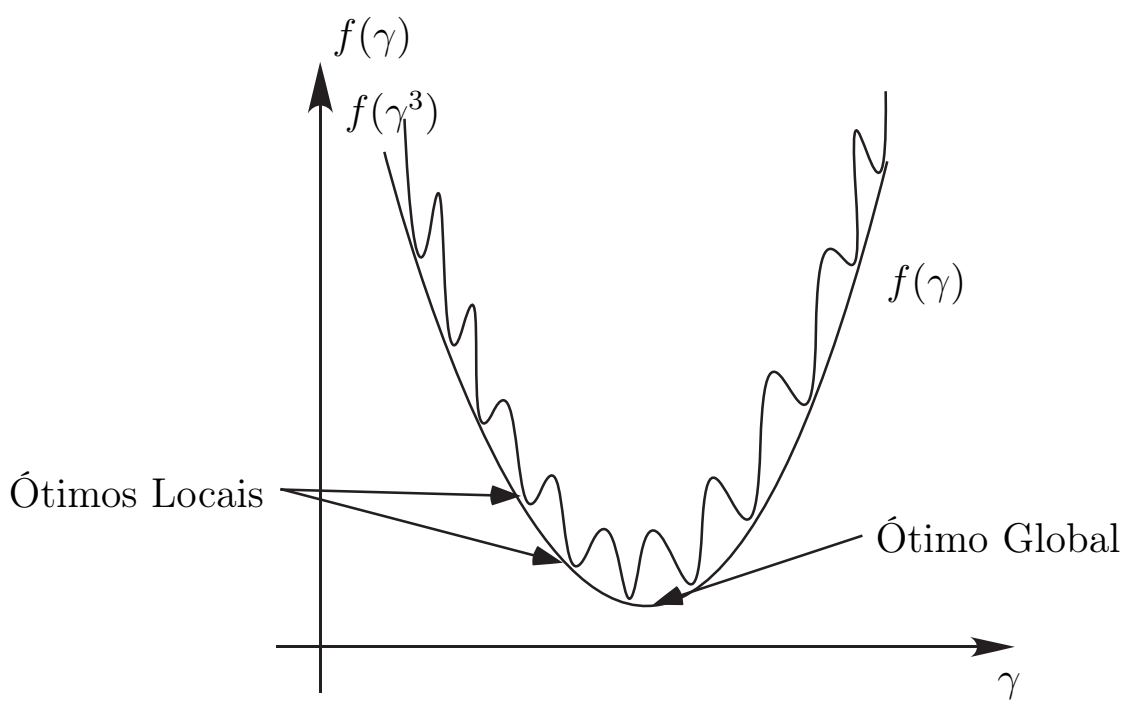

Figura 3.10: Representação esquemática da influência da penalização $p$ no problema de OT.

O método da continuação também se aplica à penalização $q$ referente à densidade.

Então, com o método da continuação, o caso em que $p<q$ do estudo sobre SIMP e RAMP feito na Seção 3.5 passa a ser válido, pois as penalizações de valores altos somente irão atuar quando a solução já estiver perto de uma solução ótima global ou de uma solução ótima local bem definida.

Apesar deste procedimento apresentar bons resultados na maioria dos casos, este método é heurístico e não é possível provar a convergência deste ou mesmo que a solução final não apresentará escala de cinza (STOLPE; SVANBERG, 2001).

\subsection{Instabilidade de Tabuleiro}

A "instabilidade de tabuleiro" é caracterizada pela formação de regiões com material (ilustrado com elementos de cor escura) e elementos sem material (ilustrado na cor branca), dispostos em forma de tabuleiro, como mostrado na Fig. 3.11.

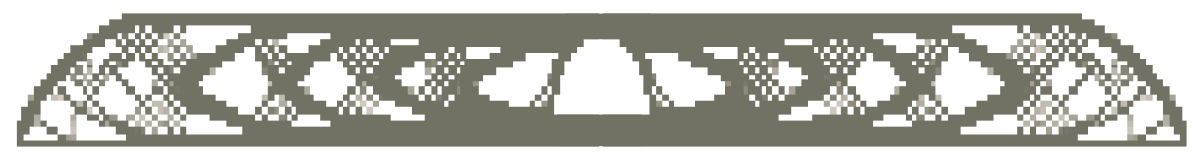

Figura 3.11: Arranjo de instabilidade de xadrez.

As publicações relacionadas com o estudo da "instabilidade de tabuleiro" (DIAZ; SIGMUND, 1995; BOURDIN, 2001; BENDSøE; SIGMUND, 2003) sugerem duas formas distintas para sua eliminação nos problemas de OT, que tradicionalmente são empregadas 
pela literatura. Uma delas é aumentar a ordem do elemento finito e a outra é utilizar métodos de filtragem ou de controle de gradientes das pseudo-densidades no domínio. A "instabilidade de tabuleiro" é indesejável na solução do problema pois não se configura numa ótima distribuição de material, mas num fenômeno que aparece devido à formulação (funções de interpolação) do elemento finito utilizado no processo de otimização.

Kikuchi, Hollister e Yoo (1997) investigaram o comportamento local do arranjo de elementos finitos dispostos em forma de "instabilidade de tabuleiro", e constataram que a ocorrência desse fenômeno nos resultados dos problemas de OT é devido às aproximações numéricas introduzidas pelo $\mathrm{MEF}$, que fazem com que o arranjo do material em forma de "instabilidade de tabuleiro" seja mais rígido ao cisalhamento do que o arranjo uniforme (material contínuo), considerando o mesmo volume de material em ambos os arranjos. Então, para se tentar contornar esse problema, pode-se aumentar a ordem do elemento, que significa aumentar o número de nós do elemento finito. Porém, é uma alternativa cara devido ao alto custo computacional. A maioria dos trabalhos que envolvem o MOT utiliza o elemento quadrilátero de 4 nós, por ter uma formulação simples de ser implementada e eficiente computacionalmente do que elementos de ordem maior, pois é importante considerar a natureza iterativa do MOT.

Além disso, foi demonstrado em estudos realizados por Jog, Haber e Bendsøe (1994), Diaz e Sigmund (1995), Jog e Haber (1996), que o problema de OT pode ser interpretado como um problema que contém um variacional misto, que envolve o campo de pseudo-densidades (grau da função pseudo-densidade no modelo de material, $p$ ) e o campo de deslocamentos (grau da função de interpolação de deslocamentos no MEF), e devido a este fator há a formação da "instabilidade de tabuleiro". Porém, dependendo do valor do fator de penalidade $p$, a "instabilidade de tabuleiro" pode ocorrer mesmo utilizando elementos de 9 nós (JOG; HABER, 1996).

Contudo, recentemente Rahmatalla, Swan e Asce (2003) demonstraram numericamente que a utilização da formulação Q4/Q4 é capaz de minimizar o efeito da "instabilidade de tabuleiro", sendo que nesse tipo de formulação as pseudo-densidades são interpoladas por nó, ou seja, a pseudo-densidade de um elemento quadrilátero é função das pseudo-densidades dos seus 4 nós. Porém, em um artigo posterior (RAHMATALLA; SWAN, 2004), os mesmos autores mostram que esta implementação apresenta um fenômeno similar à "instabilidade de tabuleiro", o qual foi denominado de "layering" e "island" ("padrão de camadas" e "ilhas", respectivamente). Entretanto, Matsui e Terada (2004) propuseram a mesma formulação e defendem, a partir de experimentos numéricos, que tal formulação é livre do problema de "instabilidade de tabuleiro". Eles a denominaram 
de Aproximação Contínua da Distribuição de Material (ACDM) que na literatura pode ser definida como "Continuous Approximation of Material Distribution" (CAMD).

\subsection{Dependência de Malha}

Seria intuitivo pensar que quanto maior fosse o número de elementos em uma malha de finitos, mais refinado seria o resultado final ótimo. Porém, o que se observa na realidade é que, com o aumento da discretização de um mesmo problema, um mesmo domínio fixo e com as mesmas condições de contorno, a topologia da estrutura ótima tende a se alterar, aumentando o número de membros da estrutura, caracterizando assim, o problema de dependência de malha. Na figura a seguir são apresentados diferentes resultados para um mesmo problema de otimização, alterando-se apenas a discretização da malha. Para o primeiro caso (Fig. 3.12(a)) utilizou-se 1200 elementos; no segundo (Fig. 3.12(b)) utilizou-se 1875 elementos; e no último resultado (Fig. 3.12(c)) utilizou-se de 3000 elementos.

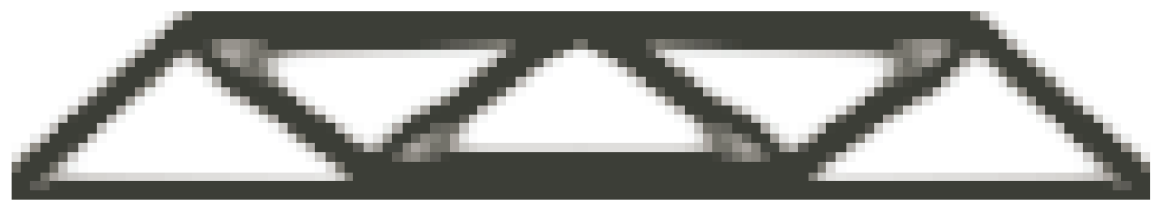

(a) 1200 elementos

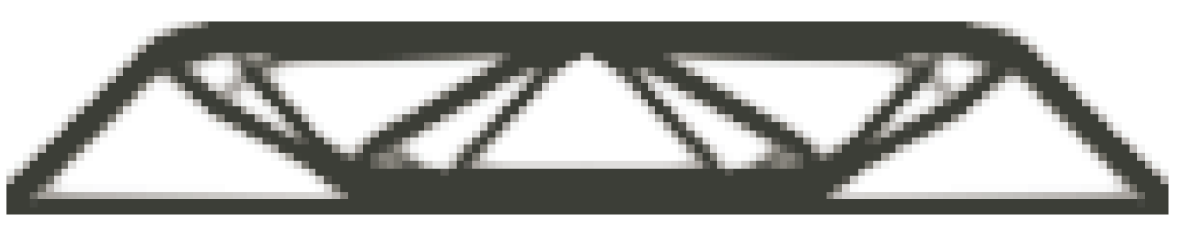

(b) 1875 elementos

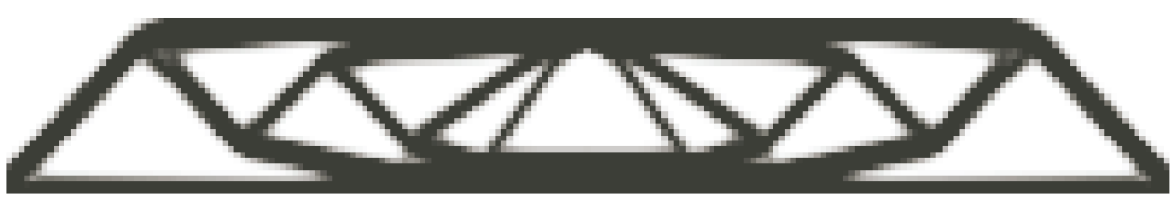

(c) 3000 elementos

Figura 3.12: Análise da dependência da malha.

O problema de dependência da malha pode ser dividido em duas categorias, conforme sua origem. Para os casos onde sempre se obtêm topologias diferentes, a medida que se 
aumenta a discretização da malha, o problema de dependência da malha é a manifestação numérica da não-existência de solução do problema. Existe também a possibilidade do problema não apresentar unicidade de solução; essa situação pode ocorrer conforme as condições de contorno aplicadas ao problema. Por exemplo, no caso de uma barra sob tração uniaxial, as soluções de uma única barra de maior diâmetro ou de diversas barras de diâmetro menor, porém com a mesma área da barra única, apresentam a mesma rigidez (SIGMUND; PETERSSON, 1998).

Uma vez que a dependência da malha é uma manifestação numérica do fato do problema discreto de parâmetros distribuídos não possuir solução, ser mal-posto, uma forma de se evitar a dependência de malha é através da relaxação desse problema, tornando-o bem-posto. Contudo, esta abordagem não é interessante para a maioria das aplicações de Engenharia, pois apresenta escala de cinza, o que impede a identificação de uma geometria.

Assim, o problema de dependência da malha está relacionado com o problema de escala de cinza, pois ao se resolver o problema na sua forma discreta ou na forma contínua com penalização, evita-se o problema de escala de cinza mas surge o problema de dependência da malha.

Uma outra forma de resolver o problema é utilizar o método de restrição de perímetro, o qual restringe o espaço de solução controlando, de certa forma, a quantidade e tamanho dos furos através do valor máximo do perímetro. Um ponto negativo da restrição de perímetro é que a de solução passa a depender também, do tamanho dos furos.

Seguindo a abordagem de restrição do espaço de solução, Sigmund e Petersson (1998) propõe uma restrição sob o gradiente espacial da densidade volumétrica, o que impede o surgimento de oscilações bruscas nos valores das pseudo-densidades. O inconveniente nesta abordagem é o grande número de restrições locais inseridas ao problema de otimização, o que em geral é difícil de ser tratado.

O problema de dependência de malha também pode ser resolvido através da utilização de filtros espaciais. Os filtros substituem a possível função não regular por uma função regularizada, que é obtida através da convolução desta com uma função suave (BOURDIN, 2001). No entanto, diferentemente dos outros métodos, os métodos de filtragem foram propostos inicialmente como uma heurística e, posteriormente, Bourdin (2001) demonstrou a existência de solução para o problema utilizando o modelo de material SIMP, associado a um determinado tipo de filtro, aplicado sobre o campo de pseudo-densidades. 
Bourdin (2001) demonstra que a aplicação do filtro sob o campo de pseudo-densidades, implica na alteração dos gradientes de modo similar ao método de filtragem dos gradientes, proposto inicialmente por Sigmund (1997). Considerando a densidade constante sob cada elemento da malha de elementos finitos, Sigmund (1997) propôs que o gradiente da função objetivo fosse alterado antes de ser inserido no algoritmo de otimização.

Swan e Kosaka (1997) propuseram uma forma de filtro baseada na alteração direta do campo de densidades. Nesta abordagem, a densidade volumétrica de cada elemento da malha é substituída por uma média ponderada entre a densidade do elemento e seus vizinhos. Assim, esse procedimento caracteriza um filtro de vizinhança fixa.

Vale notar que o filtro proposto por Swan e Kosaka (1997) também altera o gradiente a função objetivo, pois passa a acoplar a densidade de um elemento com seus vizinhos, fazendo com que a rigidez de cada elemento também seja escrita em função dos elementos vizinhos.

Baseado no filtro proposto por Swan e Kosaka (1997), Cardoso e Fonseca (1999) propuseram um filtro espacial no qual a pseudo-densidade volumétrica de cada elemento passa a depender, não apenas dos elementos vizinhos, mas de todos os elementos que se encontram dentro de um raio previamente estipulado. Neste filtro, que pode ser esquematizado como na Fig. 3.13, a vinculação entre as pseudo-densidades é feita através dos limites superiores e inferiores das pseudo-densidades volumétricas a cada passo do método de otimização, assim, os limites passam a depender do seu próprio valor, dos limites dos elementos vizinhos dentro de um raio de abrangência $R_{\max }$, do volume do elemento finito associado a cada limite dentro do filtro, da distância entre o elemento $j$ e o elemento $i$, e do número de elementos que o raio engloba.

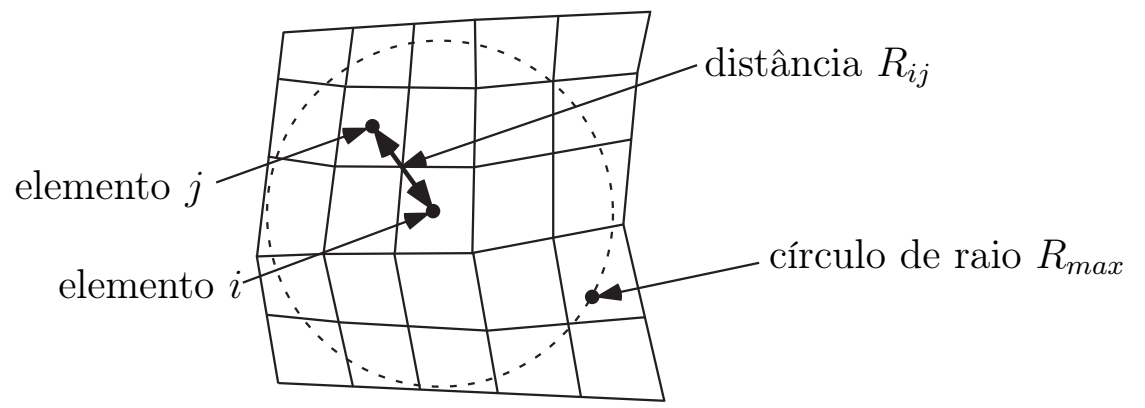

Figura 3.13: Representação esquemática do filtro aplicado sobre os limites móveis em um problema com variáveis de projeto por elemento.

A diferença entre os filtros de vizinhança fixa e os filtros espaciais é que, no primeiro, o raio de influência do filtro é dependente apenas dos elementos conectados ao elemento, e no segundo, o raio de influência é definido independentemente da conectividade da malha. 
Em termos práticos, os filtros de vizinhança fixa não são capazes de evitar o problema de dependência e malha como os filtros espaciais. Entretanto, ambos os filtros são capazes de minimizar o problema de "instabilidade de tabuleiro".

Os filtros espaciais conseguem garantir a independência da malha pois o raio de abrangência não muda conforme a malha é refinada. Ou seja, quanto mais refinada a malha, maior será o número de nós dentro do filtro. A Fig. 3.14 mostra a abrangência do filtro de raio $R_{\max }$.

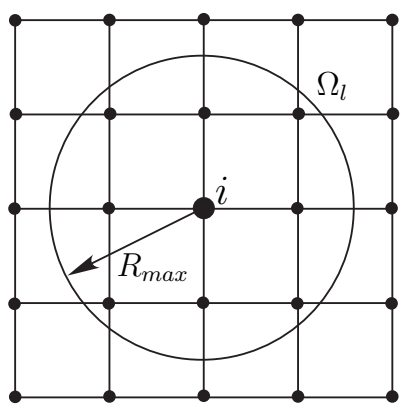

(a)

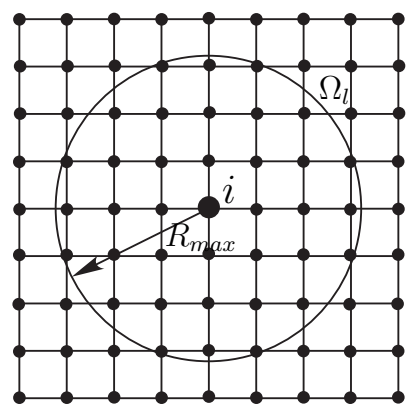

(b)

Figura 3.14: Representação de que a área de abrangência do filtro espacial não muda quando (a) é refinado para (b).

Outro ponto importante do filtro proposto por Cardoso e Fonseca (1999) é que este é aplicado sobre os limites móveis das densidades volumétricas a cada passo do processo iterativo de otimização. Esta abordagem somente é possível de ser aplicada quando se utiliza um algoritmo de otimização em que os limites móveis são explicitamente definidos, como é o caso da programação linear.

A vantagem desta abordagem é a sua capacidade de controlar a dependência de malha sem grandes alterações na formulação do problema. Porém, tem como desvantagem a influência da convergência do método de otimização, uma vez que atua sobre os limites móveis.

Para que a convergência da otimização não seja comprometida, um outro tipo de filtro espacial pode ser utilizado. Guest, Prevost e Belytschko (2004) propuseram uma técnica de projeção onde a pseudo-densidade de cada nó do domínio é uma função que depende das pseudo-densidades associadas a cada nó pertencente ao filtro e das distâncias entre o nó central $i$ e cada nó $j$ dentro do círculo de raio $R_{\max }$ (vide Fig. 3.15). Este tipo de abordagem não influi na convergência do método de otimização, pois somente está alterando as pseudo-densidades e não seus limites móveis, e ainda garante a independência 
da malha da solução ótima (GUEST; PREVOST; BELYTSCHKO, 2004).

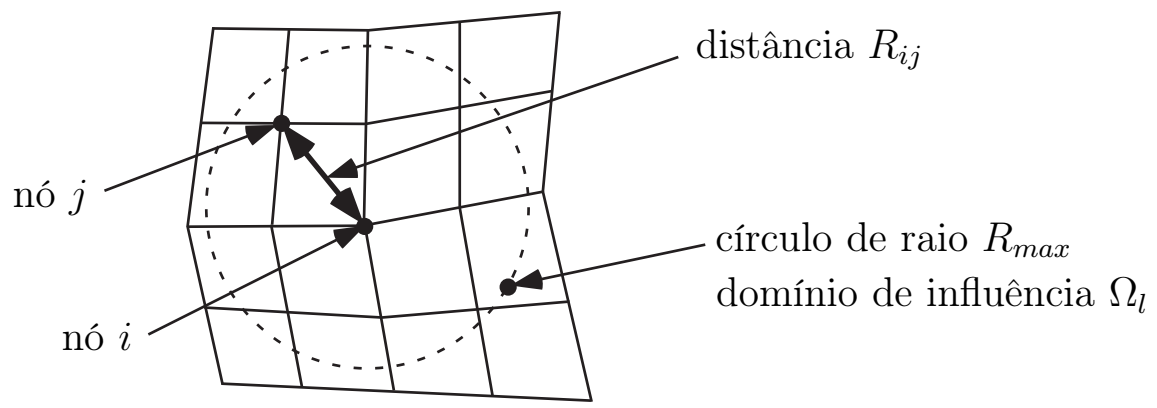

Figura 3.15: Representação esquemática do filtro em um problema com variáveis de projeto nodais.

De modo a facilitar o entendimento desse tipo de abordagem os conceitos de variável de projeto e pseudo-densidade devem ser separados. Até então, as pseudo-densidades, utilizadas no cálculo das propriedades do material, eram as próprias variáveis de projeto, as quais são utilizadas na rotina de otimização. Contudo, ao se utilizar o filtro de projeção, a pseudo-densidade passa a ser uma função linear da variável de projeto, ou seja, elas terão valores diferentes. Então, de acordo com Guest, Prevost e Belytschko (2004), a pseudo-densidade de cada nó do domínio é uma função que depende das variáveis de projeto associadas a cada nó pertencente ao filtro e das distâncias entre o nó central e cada nó dentro do raio do filtro.

Então, considerando que $d_{n}$ seja a variável de projeto e $\gamma_{n}$ o valor da pseudo-densidade associados a cada nó, tem-se que:

$$
\gamma_{n}=f\left(d_{n}\right)
$$

onde $f$ é a função de projeção definida por:

$$
\gamma_{i}=f\left(d_{j}\right)=\frac{\sum_{j \in S_{i}} d_{j} w\left(x_{j}-x_{i}\right)}{\sum_{j \in S_{i}} w\left(x_{j}-x_{i}\right)}
$$

e $S_{i}$ é o conjunto de nós dentro do domínio de influência do nó $i$ ( $\Omega_{l}$ da Fig. 3.15), o qual consiste em um círculo de raio $R_{\max }$ e centro no nó $i$. A função peso $w$ é definida por:

$$
w\left(x_{j}-x_{i}\right)=\left\{\begin{array}{cl}
\frac{R_{\max }-R_{i j}}{R_{\max }} & \text { se } x_{j} \in \Omega_{l} \\
0 & \text { caso contrário }
\end{array}\right.
$$

onde $R_{i j}$ é a distância entre os nós $j$ e $i$

$$
R_{i j}=\left\|x_{j}-x_{i}\right\|
$$




\section{FORMULAÇÃO DO PROBLEMA DE OT PARA O PROJETO DO SONOTRODO}

Como já foi dito anteriormente, o objetivo desse trabalho é definir uma formulação de otimização topológica para: uniformizar e maximizar a configuração dos deslocamentos numa dada região do sonotrodo; fazer com que a freqüência de ressonância de um certo modo seja maior ou igual à freqüência de trabalho; e garantir uma certa rigidez para o sonotrodo, a qual vibra mediante excitação harmônica de um transdutor piezelétrico de potência.

A seguir tem-se uma figura ilustrando a definição do problema de OT assim como do domínio de projeto e condições de contorno do problema.

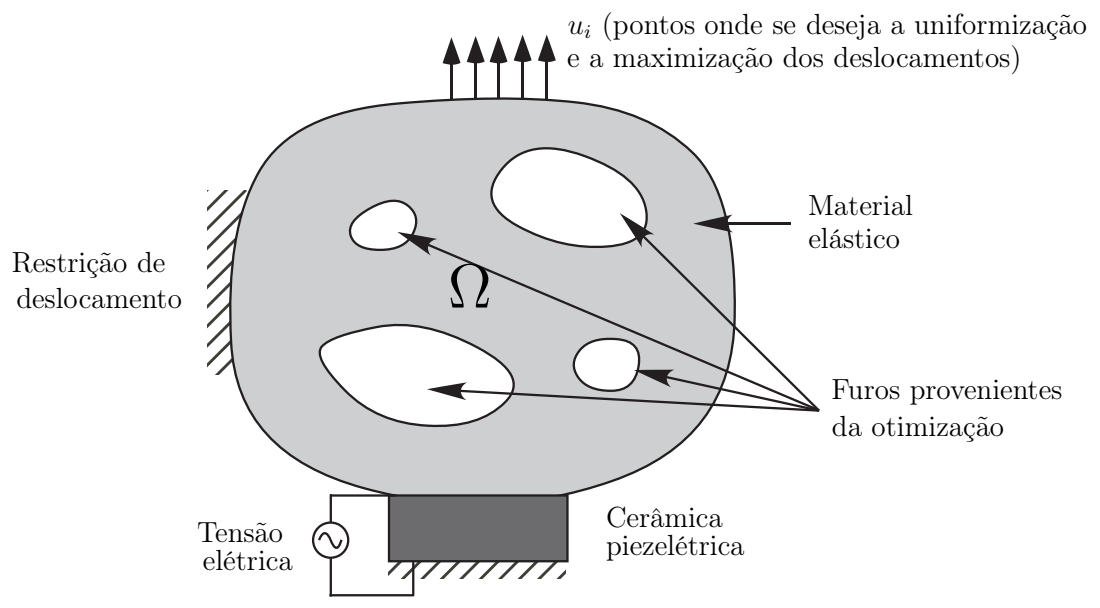

Figura 4.1: Definição do problema de OT, do domínio de projeto e condições de contorno do problema.

Assim, dado um potencial elétrico aplicado no transdutor piezelétrico, é gerada uma excitação harmônica que é transmitida ao sonotrodo. Este precisa ter rigidez o suficiente para suportar os esforços da oscilação, fornecer deslocamentos máximos e uniformes 
em uma dada região da estrutura, e possuir a freqüência de ressonância de um certo modo que seja maior ou igual à uma dada freqüência. Isso será feito utilizando-se, no problema de otimização, quatro tipos de funções objetivos diferentes: uniformização dos deslocamentos, maximização dos deslocamentos, maximização da rigidez, e maximização da diferença entre a freqüência do modo desejado e a freqüência estabelecida.

\subsection{Formulação do Problema para Uniformizar os Deslocamentos}

A uniformização é calculada pela razão entre o deslocamento mínimo e máximo $\left(U_{\min } / U_{\max }\right)$ da face de trabalho do sonotrodo, e é obtida pela minimização da soma da diferença quadrática dos deslocamentos, ou seja, é minimizada a raiz quadrada da somatória do quadrado da diferença entre o valor do deslocamento de cada ponto da região onde se deseja a uniformização $\left(u_{i}\right)$ e uma constante fornecida $(\beta)$. Assim, o equacionamento é o seguinte:

$$
L_{1}=\sqrt{\sum_{i=1}^{m}\left(u_{1 i}-\beta\right)^{2}}
$$

onde m é o número total de pontos dentro da região onde se deseja a uniformização.

Neste tipo de formulação, o objetivo é fazer com que os deslocamentos $u_{1 i}$ sejam o mais próximo possível da constante fornecida $\beta$, para que a somatória apresente um valor cada vez menor com o passar das iterações. Assim, a uniformização é obtida minimizando o seguinte problema de otimização:

$$
\begin{array}{ll}
\text { Minimizar : } & L_{1}=\sqrt{\sum_{i=1}^{m}\left(u_{1 i}-\beta\right)^{2}} \\
\text { tal que: } & \int_{V}\left[\rho\{\delta \mathbf{u}\}^{t}\{\ddot{\mathbf{u}}\}\right] d V+\int_{V}\{\delta \mathbf{S}\}^{t}\left([\mathbf{c}]^{E}\{\mathbf{S}\}-[\mathbf{e}]^{t}\{\mathbf{E}\}\right) d V=\{\delta \mathbf{u}\}^{t}\{\mathbf{f}\} \\
& \int_{V}\{\delta \mathbf{E}\}^{t}\left([\mathbf{e}]^{t}\{\mathbf{S}\}+[\varepsilon]^{S}\{\mathbf{E}\}\right) d V=\{\delta \phi\}^{t}\{\mathbf{q}\} \\
& 0 \leq d \leq 1 \\
& \Theta_{\text {lim_inf }} \leq \int_{\Omega} d d \Omega \leq \Theta_{\text {lim_sup }}
\end{array}
$$

onde $\Theta_{\text {lim_inf }}$ e $\Theta_{\text {lim_sup }}$ são os limites inferior e superior, respectivamente, de volume total permitido para a estrutura final. 


\subsection{Formulação do Problema para Maximizar os Deslocamentos}

A maximização dos deslocamentos é feita seguindo o princípio da transdução média, que é obtida aplicando-se o teorema da reciprocidade (ou teorema de Betti) proveniente da teoria da elasticidade (TIMOSHENKO; GOODIER, 1970) estendendo-o para o meio piezelétrico. Essa formulação permite obter o máximo deslocamento em direções e regiões determinadas, com a aplicação de energia elétrica no meio piezelétrico (cargas ou potenciais elétricos).

O cálculo da transdução média é realizado em dois casos de carregamento, como mostrado na Fig. 4.2. O primeiro está relacionado com a resposta dinâmica do sonotrodo $\left(\mathbf{u}_{1}\right)$ devido a aplicação dos potenciais elétricos dinâmicos $\tilde{\phi}_{1}=\phi_{1} e^{j \omega t}$ na região $\Upsilon_{\phi_{1}}$, e o segundo com a resposta dinâmica $\left(\mathbf{u}_{2}\right)$ devido a aplicação de carregamentos mecânicos dinâmicos unitários $\tilde{\mathbf{t}}_{2}=\mathbf{t}_{2} e^{j \omega t}$ na região $\Upsilon_{\mathbf{t}_{2}}$, simulando os movimentos e atuações $i$, e desta forma a transdução média relaciona potenciais elétricos com os respectivos movimentos de atuação $i$. $\phi_{1}$ e $\mathbf{t}_{2}$ são os módulos das amplitudes do potencial elétrico e do carregamento mecânico, respectivamente, sendo que $\mathbf{t}_{2}=1$, $\omega$ é a freqüência de operação, $t$ é o tempo e $j$ é o número imaginário $(j=\sqrt{-1})$.

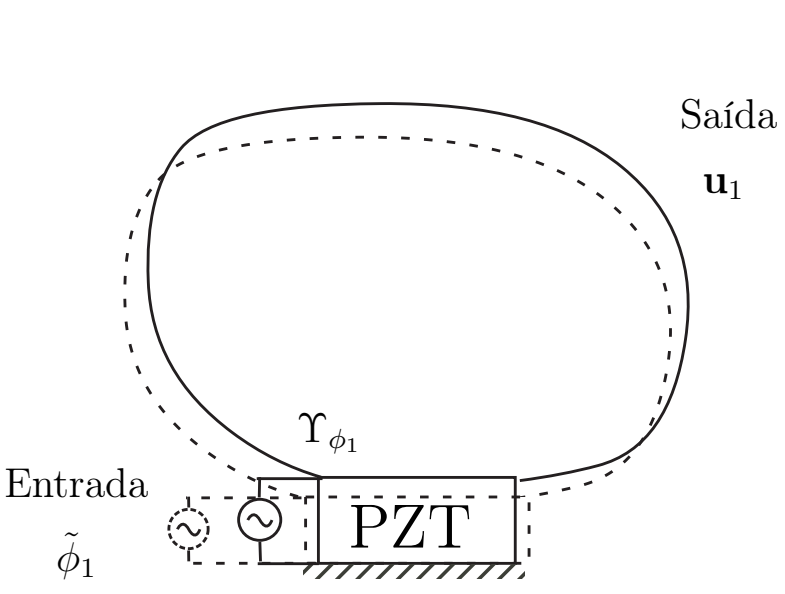

(a)

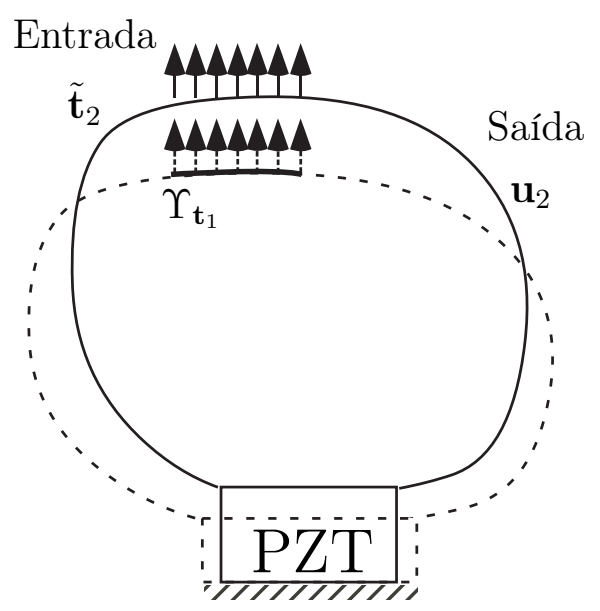

(b)

Figura 4.2: Representação dos deslocamentos gerados por (a) excitação por tensão elétrica $\tilde{\phi}_{1}$ e (b) carregamento mecânico unitário $\tilde{\mathbf{t}}_{2}$.

Então, a transdução média entre as regiões $\Upsilon_{\phi_{1}}$ e $\Upsilon_{\mathbf{t}_{2}}$ é dada por (SILVA, 2003; 
CARBONARI; SILVA; NISHIWAKI, 2005):

$$
L_{2}=\int_{\Upsilon_{\mathbf{t}_{2}}} \tilde{\mathbf{t}}_{2} \mathbf{u}_{1} d \Upsilon
$$

sendo que a dedução detalhada da transdução média é dada no Apêndice D.

Assim, a maximização da transdução média é obtida resolvendo o problema de otimização (CARBONARI; SILVA; NISHIWAKI, 2005):

$$
\begin{array}{ll}
\text { Maximizar : } & L_{2}=\int_{\Upsilon_{\mathbf{t}_{2}}} \tilde{\mathbf{t}}_{2} \mathbf{u}_{1} d \Upsilon \\
\text { tal que: } & \int_{V}\left[\rho\{\delta \mathbf{u}\}^{t}\{\ddot{\mathbf{u}}\}\right] d V+\int_{V}\{\delta \mathbf{S}\}^{t}\left([\mathbf{c}]^{E}\{\mathbf{S}\}-[\mathbf{e}]^{t}\{\mathbf{E}\}\right) d V=\{\delta \mathbf{u}\}^{t}\{\mathbf{f}\} \\
& \int_{V}\{\delta \mathbf{E}\}^{t}\left([\mathbf{e}]^{t}\{\mathbf{S}\}+[\varepsilon]^{S}\{\mathbf{E}\}\right) d V=\{\delta \phi\}^{t}\{\mathbf{q}\} \\
& 0 \leq d \leq 1 \\
& \Theta_{\text {lim_inf }} \leq \int_{\Omega} d d \Omega \leq \Theta_{\text {lim_sup }}
\end{array}
$$

sendo que $\Theta_{\text {lim_inf }}$ e $\Theta_{\text {lim_sup }}$ são os limites inferior e superior, respectivamente, de volume total permitido para a estrutura final.

\subsection{Formulação do Problema para Maximizar a Rigidez da Estrutura}

Considerando-se somente a maximização da transdução média, a solução ótima obtida pode ser uma estrutura vazia, representando uma estrutura com nenhuma rigidez e sem significado físico. Portanto, a função estrutural deve ser definida para fornecer rigidez suficiente entre $\Upsilon_{\mathbf{t}_{2}}\left(\Upsilon_{\mathbf{t}_{2}}=\Upsilon_{\mathbf{t}_{3}}\right)$ e $\Upsilon_{\phi_{1}}$, assegurando a existência de estrutura entre as regiões descritas. Ou seja, a função estrutural deve gerar rigidez o suficiente para resistir às reações sofridas durante os movimentos de atuação, de forma que o atuador mantenha a deflexão nas regiões solicitadas enquanto está sujeito a potenciais elétricos aplicados na região $\Upsilon_{\phi_{1}}$, como mostrado na Fig. 4.3 .

O problema resolvido para este caso é estático, ou seja, é utilizada somente a matriz de rigidez para o cálculo dos deslocamentos. A solução para resolver o problema estrutural estático é minimizar a flexibilidade média (ou trabalho das forças externas).

A flexibilidade média para cada movimento de atuação $i$ é calculada considerando a Fig. 4.3, onde o carregamento mecânico estático $\mathbf{t}_{3}=\mathbf{t}_{2}$ é aplicado na região $\Upsilon_{\mathbf{t}_{2}}$ e a região $\Upsilon_{\phi_{1}}$ é eletricamente aterrada, lembrando que $\mathbf{t}_{2}$ é apenas o módulo da amplitude do carregamento mecânico unitário. 


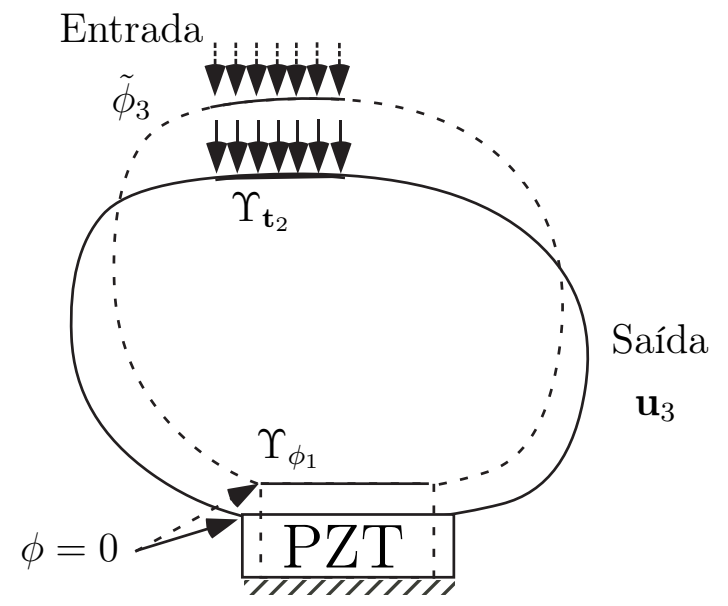

Figura 4.3: Representação dos deslocamentos gerados pelo carregamento $\mathbf{t}_{3}$.

Portanto, a flexibilidade média para cada movimento de atuação $i$ é dada por (SILVA et al., 1999; CARBONARI; SILVA; NISHIWAKI, 2005):

$$
L_{3}=\int_{\Upsilon_{\mathbf{t}_{2}}} \mathbf{t}_{3} \mathbf{u}_{3} d \Upsilon
$$

Assim, a maximização da rigidez é obtida minimizando a flexibilidade média:

$$
\begin{array}{cl}
\text { Minimizar : } & L_{3}=\int_{\Upsilon_{\mathbf{t}_{2}}} \mathbf{t}_{3} \mathbf{u}_{3} d \Upsilon \\
\text { tal que: } & \mathbf{t}_{3}=-\mathbf{t}_{2} \\
& \int_{V}\{\delta \mathbf{S}\}^{t}\left([\mathbf{c}]^{E}\{\mathbf{S}\}-[\mathbf{e}]^{t}\{\mathbf{E}\}\right) d V=\{\delta \mathbf{u}\}^{t}\{\mathbf{f}\} \\
& \int_{V}\{\delta \mathbf{E}\}^{t}\left([\mathbf{e}]^{t}\{\mathbf{S}\}+[\varepsilon]^{S}\{\mathbf{E}\}\right) d V=\{\delta \phi\}^{t}\{\mathbf{q}\} \\
& 0 \leq d \leq 1 \\
& \Theta_{\text {lim_inf }} \leq \int_{\Omega} d d \Omega \leq \Theta_{\text {lim_sup }}
\end{array}
$$

onde $\Theta_{\text {lim_inf }}$ e $\Theta_{\text {lim_sup }}$ são os limites inferior e superior, respectivamente, de volume total permitido para a estrutura final.

\subsection{Formulação do Problema para Atender a Função dos Autovalores}

O objetivo da função de autovalores é fazer com que a freqüência de ressonância associada a um certo modo de vibrar da estrutura seja maior ou igual à uma dada 
freqüência alvo. Para isso, Maeda et al. (2006) propuseram a seguinte formulação:

$$
L_{4}=\left(\frac{\lambda_{j}(d)-\lambda_{0}}{\lambda_{0}}\right)^{2}
$$

onde

$$
\begin{aligned}
\lambda_{j}(d) & =\omega_{j}^{2}(d) \\
\lambda_{0} & =\omega_{0}^{2} \\
\omega_{0} & =f_{0}
\end{aligned}
$$

sendo que $\omega_{j}$ é a freqüência de ressonância associada a um certo modo de vibrar e $\omega_{0}$ é a freqüência alvo. Contudo, essa formulação foi elaborada para que a freqüência desejada $\omega_{j}$ fique próxima à freqüência alvo $\omega_{0}$, sendo necessário minimizar $L_{4}$. Como desejamos que $\omega_{j}$ seja maior que $\omega_{0}$, então, a seguinte modificação é proposta:

$$
L_{4}=\frac{\lambda_{j}^{2}(\gamma)-\lambda_{0}^{2}}{\lambda_{0}^{2}}
$$

Assim, deve-se maximizar $L_{4}$ a fim de que $\omega_{j}(d)$ seja maior que $\omega_{0}$. Contudo, essa formulação ainda não é capaz de garantir que $\omega_{j}(d)$ seja maior que $\omega_{0}$. Ou seja, o resultado pode convergir numa estrutura em que $\omega_{j}$ seja menor que $\omega_{0}$, mesmo maximizando $L_{4}$. Portanto deve-se estabelecer um valor $\left(\omega_{1}\right)$ um pouco superior a $\omega_{0}$ de modo que $\omega_{j}(d)$ fique próximo a $\omega_{1}$, porém, não abaixo de $\omega_{0}$.

Então, para se obter uma freqüência do modo desejado próxima a uma freqüência determinada, é necessário maximizar a função $L_{4}$ da seguinte maneira:

$$
\begin{array}{ll}
\text { Maximizar : } & L_{4}=\frac{\lambda_{j}^{2}(\gamma)-\lambda_{0}^{2}}{\lambda_{0}^{2}} \\
\text { tal que: } & \int_{V}\left[\rho\{\delta \mathbf{u}\}^{t}\{\ddot{\mathbf{u}}\}\right] d V+\int_{V}\{\delta \mathbf{S}\}^{t}\left([\mathbf{c}]^{E}\{\mathbf{S}\}-[\mathbf{e}]^{t}\{\mathbf{E}\}\right) d V=0 \\
& \int_{V}\{\delta \mathbf{E}\}^{t}\left([\mathbf{e}]^{t}\{\mathbf{S}\}+[\varepsilon]^{S}\{\mathbf{E}\}\right) d V=0 \\
& 0 \leq d \leq 1 \\
& \Theta_{\text {lim_inf }} \leq \int_{\Omega} d d \Omega \leq \Theta_{\text {lim_sup }}
\end{array}
$$

onde $\Theta_{\text {lim_inf }}$ e $\Theta_{\text {lim_sup }}$ são os limites inferior e superior, respectivamente, de volume total permitido para a estrutura final. 


\subsection{Função Multi-Objetivo}

Para que se possa otimizar as quatro funções objetivo previamente descritas, deve-se combiná-las de forma a minimizar $L_{1}$ e $L_{3}$ e maximizar $L_{2}$ e $L_{4}$. Assim, é proposta a seguinte função multi-objetivo:

$$
F=w_{1} \ln L_{1}-w_{2} \ln L_{2}^{2}+w_{3} \ln L_{3}-w_{4} L_{4}
$$

onde $w_{1}, w_{2}, w_{3}$ e $w_{4}$ são os pesos que controlam a prioridade entre as funções objetivo, e seus valores serão avaliados no capítulo de resultados. A função ln é utilizada a fim de normalizar os valores das funções. A razão de se utilizar a função $L_{2}$ elevada ao quadrado $\left(L_{2}^{2}\right)$ é que, como está sendo considerado o comportamento dinâmico da estrutura, é de interesse apenas o módulo dos deslocamentos, evitando também, problemas no cálculo do logaritmo neperiano ln, o qual não aceita valores negativos. 
Então a formulação do problema de otimização topológica fica:

Minimizar : $\quad F(d)=w_{1} \ln L_{1}-w_{2} \ln L_{2}^{2}+w_{3} \ln L_{3}-w_{4} L_{4}$

$d$

tal que:

caso dinâmico (caso $\left.L_{1}\right)$ :

$\int_{V}\left[\rho\left\{\delta \mathbf{u}_{\mathbf{1}}\right\}^{t}\left\{\ddot{\mathbf{u}}_{\mathbf{1}}\right\}\right] d V+\int_{V}\{\delta \mathbf{S}\}^{t}\left([\mathbf{c}]^{E}\{\mathbf{S}\}-[\mathbf{e}]^{t}\{\mathbf{E}\}\right) d V=\left\{\delta \mathbf{u}_{\mathbf{1}}\right\}^{t}\left\{\mathbf{f}_{\mathbf{1}}\right\}$

$\int_{V}\{\delta \mathbf{E}\}^{t}\left([\mathbf{e}]^{t}\{\mathbf{S}\}+[\varepsilon]^{S}\{\mathbf{E}\}\right) d V=\left\{\delta \phi_{1}\right\}^{t}\left\{\mathbf{q}_{\mathbf{1}}\right\}$

caso dinâmico (caso $\left.L_{2}\right)$ :

$\int_{V}\left[\rho\left\{\delta \mathbf{u}_{\mathbf{2}}\right\}^{t}\left\{\ddot{\mathbf{u}}_{\mathbf{2}}\right\}\right] d V+\int_{V}\{\delta \mathbf{S}\}^{t}\left([\mathbf{c}]^{E}\{\mathbf{S}\}-[\mathbf{e}]^{t}\{\mathbf{E}\}\right) d V=\left\{\delta \mathbf{u}_{\mathbf{2}}\right\}^{t}\left\{\mathbf{f}_{\mathbf{2}}\right\}$

$\int_{V}\{\delta \mathbf{E}\}^{t}\left([\mathbf{e}]^{t}\{\mathbf{S}\}+[\varepsilon]^{S}\{\mathbf{E}\}\right) d V=\left\{\delta \phi_{2}\right\}^{t}\left\{\mathbf{q}_{\mathbf{2}}\right\}$

caso estático (caso $\left.L_{3}\right)$ :

$\int_{V}\{\delta \mathbf{S}\}^{t}\left([\mathbf{c}]^{E}\{\mathbf{S}\}-[\mathbf{e}]^{t}\{\mathbf{E}\}\right) d V=\left\{\delta \mathbf{u}_{\mathbf{3}}\right\}^{t}\left\{\mathbf{f}_{\mathbf{3}}\right\}$

$\int_{V}\{\delta \mathbf{E}\}^{t}\left([\mathbf{e}]^{t}\{\mathbf{S}\}+[\varepsilon]^{S}\{\mathbf{E}\}\right) d V=\left\{\delta \phi_{3}\right\}^{t}\left\{\mathbf{q}_{\mathbf{3}}\right\}$

caso modal (caso $\left.L_{4}\right)$ :

$\int_{V}\left[\rho\left\{\delta \mathbf{u}_{\mathbf{4}}\right\}^{t}\left\{\ddot{\mathbf{u}}_{\mathbf{4}}\right\}\right] d V+\int_{V}\{\delta \mathbf{S}\}^{t}\left([\mathbf{c}]^{E}\{\mathbf{S}\}-[\mathbf{e}]^{t}\{\mathbf{E}\}\right) d V=0$

$\int_{V}\{\delta \mathbf{E}\}^{t}\left([\mathbf{e}]^{t}\{\mathbf{S}\}+[\varepsilon]^{S}\{\mathbf{E}\}\right) d V=0$

$\mathbf{t}_{3}=-\mathbf{t}_{2}$

$0 \leq d \leq 1$

$\Theta_{\text {lim_inf }} \leq \int_{\Omega} d d \Omega \leq \Theta_{\text {lim_sup }}$

onde $\Theta_{\text {lim_inf }}$ e $\Theta_{\text {lim_sup }}$ são os limites inferior e superior, respectivamente, de volume total permitido para a estrutura final.

\subsection{Formulação Discreta das Funções Objetivo}

Considerando a formulação matricial do MEF e as equações de equilíbrio da transdução média e da flexibilidade média para cada movimento de atuação $i$, é possível reescrever e calcular numericamente a transdução média e a flexibilidade média através 
das expressões:

$$
\begin{aligned}
& L_{2}=\left\{\boldsymbol{\Gamma}_{2}\right\}^{t}\left\{\boldsymbol{\Psi}_{1}\right\} \\
& L_{3}=\left\{\boldsymbol{\Gamma}_{3}\right\}^{t}\left\{\boldsymbol{\Psi}_{3}\right\}
\end{aligned}
$$

onde

$$
\begin{aligned}
& \left\{\boldsymbol{\Gamma}_{2}\right\}=\left\{\begin{array}{c}
\mathbf{F}_{2} \\
\mathbf{0}
\end{array}\right\}=-\left\{\boldsymbol{\Gamma}_{3}\right\} \\
& \left\{\boldsymbol{\Psi}_{1}\right\}=[\tilde{\boldsymbol{\kappa}}]^{-1}\left\{\boldsymbol{\Gamma}_{1}\right\} \\
& \left\{\boldsymbol{\Psi}_{3}\right\}=[\mathbf{K}]^{-1}\left\{\boldsymbol{\Gamma}_{3}\right\}
\end{aligned}
$$

sendo que $\mathbf{F}_{2}$ é a forma discreta do vetor do carregamento mecânico $\mathbf{t}_{2}$ e $\boldsymbol{\Gamma}_{1}$ (Eq. (2.56)) é o vetor de potencial elétrico $\phi_{1}$. Deve-se lembrar que $[\mathbf{K}]$ é a matriz de rigidez estática

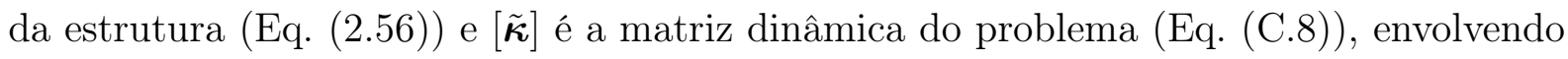
as matrizes de rigidez e massa, modificados para excitação por potencial elétrico (vide Apêndice C). Ou seja, $\left\{\boldsymbol{\Psi}_{1}\right\}$ é o vetor de deslocamentos dinâmicos mediante excitação por potencial elétrico harmônico $\left\{\boldsymbol{\Gamma}_{1}\right\}$, e $\left\{\boldsymbol{\Psi}_{3}\right\}$ é o vetor de deslocamentos estáticos mediante excitação do carregamento mecânico estático $\left\{\boldsymbol{\Gamma}_{3}\right\}$.

A função $L_{1}$ também pode ser escrita na sua forma discreta da seguinte maneira:

$$
L_{1}=\left\{\boldsymbol{\Gamma}_{2}\right\}^{t}\left(\left\{\boldsymbol{\Psi}_{1}\right\}-\{\beta\}\right)^{2}
$$

onde $\{\boldsymbol{\beta}\}$ é um vetor contendo o valor de $\beta$ em todos os termos, sendo do mesmo tamanho do vetor $\left\{\tilde{\Psi}_{1}\right\}$.

$$
\{\boldsymbol{\beta}\}=\left\{\begin{array}{c}
\beta \\
\beta \\
\beta \\
\vdots \\
\beta \\
\beta
\end{array}\right\}
$$

\subsection{Problema de OT na Forma Discreta}

As funções objetivo $L_{1}, L_{2}$ e $L_{3}$ são implementadas conforme apresentadas na sua forma discreta na Seção 4.6. A função $L_{4}$ não possui forma discreta, pois ela não é calculada por integral ou somatória. Com isso, a formulação do problema de otimização 
topológica é implementado da seguinte maneira:

$$
\begin{array}{cl}
\text { Maximizar: } & F(d)=w_{1} \ln L_{1}-w_{2} \ln L_{2}^{2}+w_{3} \ln L_{3}-w_{4} L_{4} \\
\text { tal que: } \quad & \left\{\boldsymbol{\Gamma}_{3}\right\}=-\left\{\boldsymbol{\Gamma}_{2}\right\} \\
& {[\tilde{\boldsymbol{\kappa}}]\left\{\boldsymbol{\Psi}_{1}\right\}=\left\{\boldsymbol{\Gamma}_{1}\right\}} \\
& {[\tilde{\boldsymbol{\kappa}}]\left\{\boldsymbol{\Psi}_{2}\right\}=\left\{\boldsymbol{\Gamma}_{2}\right\}} \\
& {[\mathbf{K}]\left\{\boldsymbol{\Psi}_{3}\right\}=\left\{\boldsymbol{\Gamma}_{3}\right\}} \\
& \left([\overline{\mathbf{K}}]-\omega_{j}^{2}[\overline{\mathbf{M}}]\right)\{\mathbf{W}\}=0 \\
& 0<d_{\text {min }} \leq d_{i} \leq 1 \quad i=1, \ldots, N_{e} \\
& \Theta_{\text {lim_inf }} \leq \sum_{i=1}^{N_{e}} d_{i} V_{i}^{0} \leq \Theta_{\text {lim_sup }}
\end{array}
$$

lembrando que $w_{1}, w_{2}, w_{3}$ e $w_{4}$ são os pesos das funções objetivo, $\left\{\boldsymbol{\Gamma}_{1}\right\}$ é o vetor de potencial elétrico dinâmico, $\left\{\boldsymbol{\Gamma}_{2}\right\}$ é o vetor de carregamento mecânico unitário dinâmico, $\left\{\boldsymbol{\Gamma}_{3}\right\}$ é o vetor de carregamento mecânico unitário estático, $[\tilde{\boldsymbol{\kappa}}]$ é a matriz dinâmica do problema considerando excitação por potencial elétrico (Eq. (C.8)), [K] é a matriz de rigidez estática, $[\overline{\mathbf{K}}]$ e $[\overline{\mathbf{M}}]$ são as matrizes de rigidez e massa considerando as condições de contorno para o cálculo das freqüências de ressonância $\omega_{j}$ e modos de vibrar $\{\mathbf{W}\}$. Além disso, $d_{\text {min }}$ é inserido na restrição de caixa das variáveis de projeto para evitar instabilidades numéricas, e $\sum_{i=1}^{N_{e}} d_{i} V_{i}^{0}$ é a forma discreta do cálculo do volume $\int_{\Omega} d d \Omega$, sendo que $\Theta_{\text {lim_inf }}$ e $\Theta_{\text {lim_sup }}$ são os limites inferior e superior, respectivamente, de volume total permitido para a estrutura final.

\subsection{Cálculo de Sensibilidades}

Os gradientes (ou derivadas) da função objetivo e restrições são chamados de sensibilidades do problema de otimização. O cálculo desses gradientes é importante devido à necessidade da linearização da função objetivo em relação as variáveis do problema (que são relacionadas a cada nó do domínio discretizado), como visto na implementação numérica da rotina de Programação Linear Seqüencial (PLS). Diante desta necessidade, neste item são apresentados os gradientes da função objetivo apresentados anteriormente para a formulação da otimização topológica de sonotrodos para transdutores piezelétricos. O cálculo dos gradientes é demonstrado aqui utilizando o método adjunto (HAFTKA; GURDAL; KAMAT, 1990), e é feito segundo a regra da cadeia, pois todas as funções, restrições, matrizes e vetores dependem das pseudo-densidades de cada nó, e estas sim, dependem das variáveis de projeto. Então, como exemplo da função multi-objetivo $F$ 
(Eq. (4.9)), sua derivada fica:

$$
\frac{\partial F}{\partial d_{i}}=\frac{\partial F}{\partial \gamma_{n}} \frac{\partial \gamma_{n}}{\partial d_{i}}
$$

onde $\gamma_{n}$ são as pseudo-densidades e $d_{i}$ as variáveis de projeto. As demais sensibilidades podem ser obtidas por processo análogo.

Assim, o gradiente da função $F$ (Eq. (4.9)) relativo à pseudo-densidade $\gamma_{n}$ é dado por:

$$
\frac{\partial F}{\partial \gamma_{n}}=\frac{w_{1}}{L_{1}} \frac{\partial L_{1}}{\partial \gamma_{n}}-2 \frac{w_{2}}{L_{2}} \frac{\partial L_{2}}{\partial \gamma_{n}}+\frac{w_{3}}{L_{3}} \frac{\partial L_{3}}{\partial \gamma_{n}}-w_{4} \frac{\partial L_{4}}{\partial \gamma_{n}}
$$

onde $\partial L_{1} / \partial \gamma_{n}, \partial L_{2} / \partial \gamma_{n}, \partial L_{3} / \partial \gamma_{n}$ e $\partial L_{4} / \partial \gamma_{n}$ são as derivadas das funções $L_{1}, L_{2}, L_{3}$ e $L_{4}$, respectivamente, em relação às pseudo-densidades, e o subscrito $n$ indica o número da pseudo-densidade.

Os gradientes das funções $L_{2}$ e $L_{3}$ podem ser calculados derivando-se a forma matricial em que foram escritas nas Eqs. (4.10) e (4.11).

$$
\begin{aligned}
& \frac{\partial L_{2}}{\partial \gamma_{n}}=\left\{\boldsymbol{\Gamma}_{2}\right\}^{t} \frac{\partial\left\{\boldsymbol{\Psi}_{1}\right\}}{\partial \gamma_{n}} \\
& \frac{\partial L_{3}}{\partial \gamma_{n}}=\left\{\boldsymbol{\Gamma}_{3}\right\}^{t} \frac{\partial\left\{\boldsymbol{\Psi}_{3}\right\}}{\partial \gamma_{n}}
\end{aligned}
$$

A derivada do vetor $\left\{\boldsymbol{\Psi}_{1}\right\}$ é obtida derivando-se, pela regra da cadeia, a Eq. (2.59). O desenvolvimento detalhado desta derivada é dado no Apêndice C.

Então, $\partial\left\{\boldsymbol{\Psi}_{1}\right\} / \partial \gamma_{n}$ é dado por (Eq. (C.12)):

$$
\frac{\partial\left\{\boldsymbol{\Psi}_{1}\right\}}{\partial \gamma_{n}}=-[\tilde{\boldsymbol{\kappa}}]^{-1} \frac{\partial[\tilde{\boldsymbol{\kappa}}]}{\partial \gamma_{n}}\left\{\boldsymbol{\Psi}_{1}\right\}
$$

onde $[\tilde{\boldsymbol{\kappa}}]$ é a matriz dinâmica ajustada para excitação por potencial elétrico e é dada pela Eq. (C.8). Sua derivada $\left(\partial[\tilde{\boldsymbol{\kappa}}] / \partial \gamma_{n}\right)$ é dada pela Eq. (C.9), incluindo as derivadas das matrizes de massa $\left(\partial[\mathbf{M}] / \partial \gamma_{n}\right)$ e de rigidez $\left(\partial[\mathbf{K}] / \partial \gamma_{n}\right)$.

A derivada do vetor $\left\{\Psi_{3}\right\}$ é obtida de modo análogo ao processo de obtenção da derivada do vetor $\left\{\boldsymbol{\Psi}_{1}\right\}$, contudo deve ser considerada a situação estática onde é utilizada somente a matriz de rigidez $([\mathbf{K}])$ e sem considerar a excitação por potencial elétrico, o que resulta em:

$$
\frac{\partial\left\{\mathbf{\Psi}_{3}\right\}}{\partial \gamma_{n}}=-[\mathbf{K}]^{-1} \frac{\partial[\mathbf{K}]}{\partial \gamma_{n}}\left\{\boldsymbol{\Psi}_{3}\right\}
$$

As derivadas das matrizes de rigidez e massa devem ser obtidas considerando-se o modelo de material utilizado. Neste trabalho foi utilizado o modelo SIMP tanto para 
a matriz de rigidez quanto para a matriz de massa, porém sempre com penalização da densidade $q$ maior que a penalização da rigidez $p$ e utilizando o método da continuação.

Sabendo-se do MEF que as matrizes de rigidez e massa podem ser escritas da seguinte forma:

$$
[\mathbf{K}]=E(\mathrm{x})\left[\mathbf{K}^{0}\right] \quad ; \quad[\mathbf{M}]=\rho(\mathrm{x})\left[\mathbf{M}^{0}\right]
$$

e substituindo $\rho(\gamma)$ da Eq. (3.5) e $E(\gamma)$ da Eq. (3.8) na Eq. (4.24) e derivando-as, tem-se:

$$
\begin{aligned}
& \frac{\partial[\mathbf{K}]}{\partial \gamma_{n}}=p \gamma(\mathrm{x})^{p-1} \frac{\partial \gamma(\mathrm{x})}{\partial \gamma_{n}}\left[\mathbf{K}^{0}\right] \\
& \frac{\partial[\mathbf{M}]}{\partial \gamma_{n}}=q \gamma(\mathrm{x})^{q-1} \frac{\partial \gamma(\mathrm{x})}{\partial \gamma_{n}}\left[\mathbf{M}^{0}\right]
\end{aligned}
$$

onde $\left[\mathbf{K}^{0}\right]$ e $\left[\mathbf{M}^{0}\right]$ são as matrizes de rigidez e massa, respectivamente, sem considerar as propriedades dos materiais.

Para se implementar o gradiente da função $L_{1}$ é necessário fazer uma manipulação vetorial para que o cálculo seja simplificado. Então, a Eq. (4.15) fica:

$$
\frac{\partial L_{1}}{\partial \gamma_{n}}=-2\left\{\left[\overline{\boldsymbol{\Gamma}}_{2}\right]\left(\left\{\boldsymbol{\Psi}_{1}\right\}-\{\boldsymbol{\beta}\}\right)\right\}^{t}[\tilde{\boldsymbol{\kappa}}]^{-1} \frac{\partial[\tilde{\boldsymbol{\kappa}}]}{\partial \gamma_{n}}\left\{\boldsymbol{\Psi}_{1}\right\}
$$

onde

$$
\left[\overline{\boldsymbol{\Gamma}}_{2}\right]=\left[\begin{array}{ccccc}
\Gamma_{2_{1}} & 0 & 0 & 0 & 0 \\
0 & \Gamma_{2_{2}} & 0 & 0 & 0 \\
0 & 0 & \Gamma_{2_{3}} & 0 & 0 \\
0 & 0 & 0 & \ddots & 0 \\
0 & 0 & 0 & 0 & \Gamma_{2_{n}}
\end{array}\right]
$$

lembrando que $[\tilde{\boldsymbol{\kappa}}]$ é a matriz dinâmica considerando excitação por potencial elétrico dada pela Eq. (C.8). Ou seja, a multiplicação da matriz $\left[\overline{\boldsymbol{\Gamma}}_{2}\right]$ pelo vetor $\left\{\boldsymbol{\Psi}_{1}\right\}-\{\boldsymbol{\beta}\}$ resulta num vetor contendo os valores $u_{1 i}-\beta$ dos pontos onde se deseja a otimização e o valor " 0 " (zero) no restante do vetor, sendo $u_{1 i}$ o valor do deslocamento gerado pela excitação por potencial elétrico nos pontos da otimização. Então, pode ser feita a seguinte simplificação:

$$
\left\{\left[\overline{\boldsymbol{\Gamma}}_{2}\right]\left(\left\{\boldsymbol{\Psi}_{1}\right\}-\{\beta\}\right)\right\}^{t}=\left\{\tilde{\boldsymbol{\Gamma}}_{12}\right\}^{t}
$$

possibilitando fazer o seguinte cálculo:

$$
\begin{aligned}
& {[\tilde{\boldsymbol{\kappa}}]\{\boldsymbol{\Lambda}\}=\left\{\tilde{\boldsymbol{\Gamma}}_{12}\right\} \Rightarrow\{\boldsymbol{\Lambda}\}=[\tilde{\boldsymbol{\kappa}}]^{-1}\left\{\tilde{\boldsymbol{\Gamma}}_{12}\right\} \Rightarrow} \\
& \Rightarrow\{\boldsymbol{\Lambda}\}^{t}=\left([\tilde{\boldsymbol{\kappa}}]^{-1}\left\{\tilde{\boldsymbol{\Gamma}}_{12}\right\}\right)^{t} \Rightarrow\{\boldsymbol{\Lambda}\}^{t}=\left\{\tilde{\boldsymbol{\Gamma}}_{12}\right\}^{t}[\tilde{\boldsymbol{\kappa}}]^{-1}
\end{aligned}
$$


sendo que, para fins de implementação, $\{\boldsymbol{\Lambda}\}$ é o vetor de "deslocamentos" da estrutura resultante da aplicação da "força fictícia" $\left\{\tilde{\boldsymbol{\Gamma}}_{12}\right\}$. Ou seja, os termos $u_{1 i}-\beta$ dos pontos onde se deseja a otimização se tornam a excitação do sub-problema da Eq. (4.30), e geram os deslocamentos representados por $\{\boldsymbol{\Lambda}\}$. Todavia, fisicamente, esses deslocamentos são utilizados somente para auxiliar no cálculo da implementação.

Então, a Eq. (4.27) fica:

$$
\frac{\partial L_{1}}{\partial \gamma_{n}}=-2\{\boldsymbol{\Lambda}\}^{t} \frac{\partial[\tilde{\boldsymbol{\kappa}}]}{\partial \gamma_{n}}\left\{\boldsymbol{\Psi}_{1}\right\}
$$

É também necessário calcular o gradiente da função $L_{4}$, que é obtido derivando a Eq. (4.8), que fica:

$$
\frac{\partial L_{4}}{\partial \gamma_{n}}=2 \frac{\lambda_{j}}{\lambda_{0}^{2}} \frac{\partial \lambda_{j}}{\partial \gamma_{n}}
$$

sendo que a sensibilidade do autovalor $\frac{\partial \lambda_{j}}{\partial \gamma_{n}}$ é obtida derivando a Eq. (2.61), e é dada por (SILVA; KIKUCHI, 1999):

$$
\frac{\partial \lambda}{\partial \gamma_{n}}=\frac{\{\mathbf{W}\}^{t}\left(\frac{\partial[\overline{\mathbf{K}}]}{\partial \gamma_{n}}-\lambda \frac{\partial[\overline{\mathbf{M}}]}{\partial \gamma_{n}}\right)\{\mathbf{W}\}}{\{\mathbf{W}\}^{t}[\overline{\mathbf{M}}]\{\mathbf{W}\}}
$$

sendo $\lambda$ e $\{\mathbf{W}\}$, o autovalor e autovetor, respectivamente, calculados anteriormente pela Eq. (2.61). $[\overline{\mathbf{K}}]$ e $[\overline{\mathbf{M}}]$ são as matrizes de rigidez e massa reduzidas para as condições de contorno da análise modal para freqüência de ressonância onde todos os eletrodos são aterrados, como explicado na seção 2.5, e suas derivadas são dadas por:

$$
\begin{aligned}
& \frac{\partial[\overline{\mathbf{K}}]}{\partial \gamma_{n}}=p \gamma(\mathbf{x})^{p-1} \frac{\partial \gamma(\mathbf{x})}{\partial \gamma_{n}}\left[\overline{\mathbf{K}}^{0}\right] \\
& \frac{\partial[\overline{\mathbf{M}}]}{\partial \gamma_{n}}=q \gamma(\mathbf{x})^{q-1} \frac{\partial \gamma(\mathbf{x})}{\partial \gamma_{n}}\left[\overline{\mathbf{M}}^{0}\right]
\end{aligned}
$$

onde $\left[\overline{\mathbf{K}}^{0}\right]$ e $\left[\overline{\mathbf{M}}^{0}\right]$ são as matrizes de rigidez e massa, respectivamente, adaptadas para a análise modal, sem considerar as propriedades dos materiais.

Por fim, a sensibilidade da pseudo-densidade $\gamma_{n}$ em relação à variável de projeto $d_{i}$ é dada pela derivada da Eq. (3.12):

$$
\frac{\partial \gamma_{n}}{\partial d_{i}}=\frac{\omega\left(x_{i}-x_{n}\right)}{\sum_{j \in \Omega_{n}} \omega\left(x_{j}-x_{n}\right)}
$$

onde $d_{i}$ são as variáveis de projeto, $\gamma_{n}$ são as pseudo-densidades, e o índice $j$ indicam as variáveis de projeto das quais as pseudo-densidades $\gamma_{n}$ dependem. 


\section{IMPLEMENTAÇÃO NUMÉRICA}

O problema de otimização, apresentado no capítulo 4, pode ser resolvido numericamente através de um algoritmo computacional que combina o método de otimização de programação linear (PLS) (DANTZIG, 1963; HAFTKA; GURDAL; KAMAT, 1990; YANG R.J., 1994) com o método numérico de análise por elementos finitos (MEF).

Pode-se resolver os problemas de otimização estrutural utilizando diversas aproximações distintas, como por exemplo, o Critério de Optimalidade (CO) (HASSANI; HINTON, 1998a; BENDSøE; SIGMUND, 2003), o método de PLS, o Método de movimento assintótico (MMA) (SVANBERG, 1987; BRUYNEEL; DUYSINX; FLEURY, 2002) e o Programação Quadrática Sequencial (PQS) (HAFTKA; GURDAL; KAMAT, 1990).

Neste trabalho será utilizado o método PLS por ser um método genérico, ou seja, os métodos genéricos consistem em métodos de otimização que são baseados na chamada teoria de programação matemática e podem ser aplicados em vários problemas de otimização, seja no projeto de estruturas ou em outros ramos de aplicação. O PLS é uma rotina que resolve seqüencialmente vários sub-problemas lineares na busca da solução do problema não-linear, o qual geralmente possui uma função objetivo complexa, um grande número de variáveis de projeto e várias restrições.

\subsection{Modelo de Material}

O modelo de material adotado no projeto é baseado no SIMP (BENDSøE; SIGMUND, 2003) para o módulo de elasticidade e para a densidade (Eq. (3.5)).

Esse modelo é implementado utilizando a abordagem CAMD (JOG; HABER, 1996; RAHMATALLA; SWAN; ASCE, 2003; RAHMATALLA; SWAN, 2004; MATSUI; TERADA, 2004) de pseudo-densidades nodais:

$$
\gamma(\mathbf{x})=\sum_{i=1}^{n_{d}} \gamma_{i} N_{i}(\mathbf{x})
$$


onde $\gamma_{i}$ é a pseudo-densidade definida para cada nó pertencente ao domínio da estrutura, $N_{i}$ são as funções de forma (ver Eq. (2.16), definida na seção 2.3.1) e $n_{d}$ é o número de nós de cada elemento finito, que neste caso, $n_{d}=4$.

\subsection{Implementação Numérica do MEF}

As matrizes de massa e rigidez obtidas pelo MEF são dadas por integrais (Eqs. (2.44) à (2.47) para o elemento axissimétrico e Eqs. (2.49) à (2.52) para o EPTM) e são calculadas por integração numérica utilizando-se a Quadratura de Gauss. Como exemplo, a matriz de massa pode ser implementada numericamente da seguinte forma, para o elemento axissimétrico:

$$
\left[\mathbf{M}_{u u}\right]_{e}=2 \pi \sum_{k=1}^{2} \sum_{l=1}^{2} w_{k} w_{l} \rho(\mathbf{x})\left[\mathbf{N}_{u}\left(\xi_{k}, \eta_{l}\right)\right]^{t}\left[\mathbf{N}_{u}\left(\xi_{k}, \eta_{l}\right)\right]\left(\sum_{i=1}^{4} r_{i} N_{i}\left(\xi_{k}, \eta_{l}\right)\right) \operatorname{det}[\mathbf{J}]
$$

e para o elemento EPTM:

$$
\left[\mathbf{M}_{u u}\right]_{e}=h_{e} \sum_{k=1}^{2} \sum_{l=1}^{2} w_{k} w_{l} \rho(\mathbf{x})\left[\mathbf{N}_{u}\left(\xi_{k}, \eta_{l}\right)\right]^{t}\left[\mathbf{N}_{u}\left(\xi_{k}, \eta_{l}\right)\right] \operatorname{det}[\mathbf{J}]
$$

onde $\left[\mathbf{N}_{u}\left(\xi_{k}, \eta_{l}\right)\right]$ é a matriz de funções de forma avaliada para cada ponto $\left(\xi_{k}, \eta_{l}\right)$ da quadratura de Gauss, $w_{k}$ e $w_{l}$ são os pesos associados aos pontos de Gauss que, para o caso de 2 pontos, tem o valor um, $r_{i}$ é a coordenada de cada nó e $N_{i}$ é a função de forma de cada nó do elemento.

É também necessário levar em consideração a diferença entre o elemento otimizável e o não-otimizável. Para o elemento não-otimizável, faz-se $\rho(\mathbf{x})=\rho_{0}$ para as Eqs. (5.2) e (5.3). Para o elemento otimizável, deve-se considerar o modelo de material utilizando a abordagem CAMD:

$$
\rho(\mathbf{x})=\left(\sum_{i=1}^{n_{d}} \gamma_{i} N_{i}(\mathbf{x})\right)^{q} \rho_{0}
$$

lembrando que ao utilizar a função de projeção ((GUEST; PREVOST; BELYTSCHKO, 2004)) descrita na seção 3.8, as pseudo-densidades $\gamma_{i}$ passam a ser uma função que depende das variáveis de projeto $d_{j}$. A função de projeção é definida por (Eq. (3.12)):

$$
\gamma_{i}=\frac{\sum_{j \in S_{i}} d_{j} w\left(x_{j}-x_{i}\right)}{\sum_{j \in S_{i}} w\left(x_{j}-x_{i}\right)}
$$


onde

$$
\begin{gathered}
w\left(x_{j}-x_{i}\right)=\left\{\begin{array}{cl}
\frac{R_{\max }-R_{i j}}{R_{\max }} & \text { se } x_{j} \in \Omega_{l} \\
0 & \text { caso contrário }
\end{array}\right. \\
R_{i j}=\left\|x_{j}-x_{i}\right\|
\end{gathered}
$$

e $S_{i}$ é o conjunto de nós dentro do domínio de influência do nó $i$ ( $\Omega_{l}$ da Fig. 5.1), o qual consiste em um círculo de raio $R_{\max }$ e centro no nó $i$.

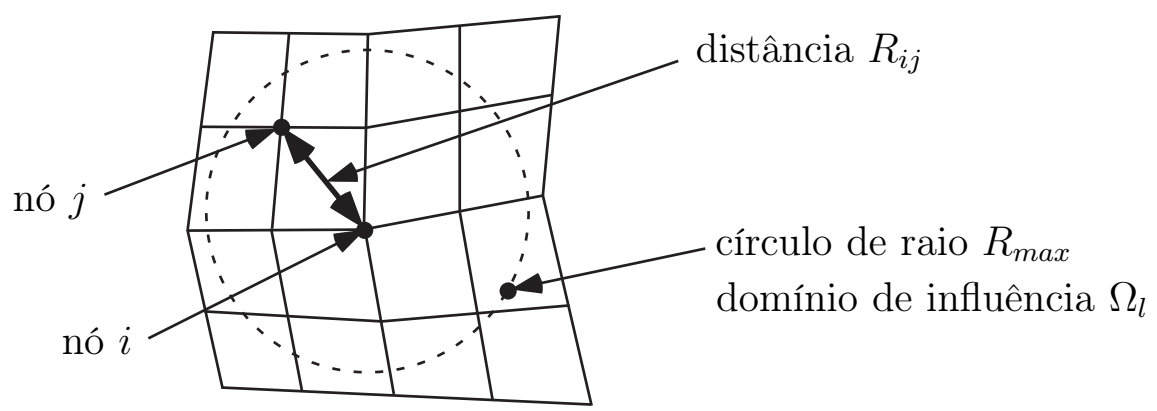

Figura 5.1: Representação esquemática do filtro em um problema com variáveis de projeto nodais.

Então, as matrizes de massa para o elemento axissimétrico e para o elemento EPTM são calculadas substituindo-se a Eq. (5.4) nas Eqs. (5.2) e (5.3).

As matrizes de rigidez elástica, piezelétrica e dielétrica são obtidas fazendo-se procedimento análogo.

\subsection{Programação Linear Seqüencial}

O PLS adotado neste trabalho permite lidar com um grande número de variáveis de projeto $\left(d_{n}\right)$ e diferentes funções objetivo e restrições (VANDERPLAATS, 1984; HAFTKA; GURDAL; KAMAT, 1990). A programação linear (YANG R.J., 1994) resolve um problema de otimização em que a função objetivo e as restrições são funções lineares em relação às variáveis do problema, ou seja, o problema de ter a seguinte forma:

$$
\begin{array}{cl}
\text { Maximizar: } & F(d)=a_{1} d_{1}+a_{2} d_{2}+\cdots+a_{n} d_{n} \\
d_{1, \ldots, n} & \\
\text { Sujeita à : } & g_{1}(d)=b_{1} d_{1}+b_{2} d_{2}+\cdots+b_{n} d_{n} \leq c \\
& g_{2}(d)=c_{1} d_{1}+c_{2} d_{2}+\cdots+c_{n} d_{n} \geq d \\
& g_{3}(d)=d_{1} d_{1}+d_{2} d_{2}+\cdots+d_{n} d_{n}=e
\end{array}
$$


Embora a função objetivo do problema de otimização mostrado no capítulo 4 (seção 4.5) não seja linear em relação às variáveis do problema $\left(d_{j}\right)$, o problema pode ser linearizado calculando-se a Série de Taylor de $1^{\mathrm{a}}$ ordem da função objetivo. Sabe-se do cálculo diferencial que a série de Taylor pode representar uma função $f(d)$, para $d$ próximo de um valor $d_{0}$, a menos de um erro $e(d)$, como ilustra a equação abaixo:

$$
f(d)=f\left(d_{0}\right)+\frac{\partial f}{\partial d}\left(d-d_{0}\right)+\frac{\partial^{2} f}{\partial d^{2}} \frac{\left(d-d_{0}\right)^{2}}{2 !}+\cdots+\frac{\partial^{J} f}{\partial d^{J}} \frac{\left(d-d_{0}\right)^{J}}{J !}+e(d)
$$

onde $f\left(d_{0}\right)$ é o valor da função para o valor atual da variável e $J$ significa a ordem da derivada. Para uma função com $n$ variáveis, teríamos:

$$
\begin{aligned}
f\left(d_{1}, \ldots, d_{n}\right)= & f\left(d_{0_{1}}, \ldots, d_{0_{n}}\right)+\frac{\partial f}{\partial d_{1}}\left(d_{1}-d_{0_{1}}\right)+\cdots+\frac{\partial^{J} f}{\partial d_{1}^{J}} \frac{\left(d_{1}-d_{0_{1}}\right)^{J}}{J !}+ \\
& +\frac{\partial f}{\partial d_{2}}\left(d_{2}-d_{0_{2}}\right)+\cdots+\frac{\partial^{J} f}{\partial d_{2}^{J}} \frac{\left(d_{2}-d_{0_{2}}\right)^{J}}{J !}+\cdots+ \\
& +\frac{\partial f}{\partial d_{n}}\left(d_{n}-d_{0_{n}}\right)+\cdots+\frac{\partial^{J} f}{\partial d_{n}^{J}} \frac{\left(d_{n}-d_{0_{n}}\right)^{J}}{J !}+e\left(d_{1}, d_{2}, \ldots, d_{n}\right)
\end{aligned}
$$

Os termos com derivada de segunda ordem ou superior são desprezados, obtendo uma linearização da função objetivo, para ser utilizada na PLS. Então, podemos linearizar a função multi-objetivo $(F)$, como:

$$
F=F_{0}+\frac{\partial F}{\partial d_{1}}\left(d_{1}-d_{0_{1}}\right)+\frac{\partial F}{\partial d_{2}}\left(d_{2}-d_{0_{2}}\right)+\cdots+\frac{\partial F}{\partial d_{n}}\left(d_{n}-d_{0_{n}}\right)
$$

Separando os termos constantes dos termos dependentes das variáveis da Eq. (5.10), temos:

$$
F=\underbrace{F_{0}-\frac{\partial F}{\partial d_{1}} d_{0_{1}}-\cdots-\frac{\partial F}{\partial d_{n}} d_{0_{n}}}_{\text {Constantes }}+\underbrace{\frac{\partial F}{\partial d_{1}} d_{1}+\cdots+\frac{\partial F}{\partial d_{n}} d_{n}}_{\text {Variáveis }}
$$

Os termos constantes não influenciam no processo de otimização da função, portanto, podem ser passados para o lado esquerdo da Eq. (5.10). Desta maneira a função a ser maximizada na PL é dada por:

$$
\frac{\partial F}{\partial d_{1}} d_{1}+\cdots+\frac{\partial F}{\partial d_{n}} d_{n}=F^{\text {linear }}
$$

onde:

$$
F^{\text {linear }}=F-\left(F_{0}-\frac{\partial F}{\partial d_{1}} d_{0_{1}}-\cdots-\frac{\partial F}{\partial d_{n}} d_{0_{n}}\right)
$$


Observe que agora a função a ser maximizada (Eq. (5.12)) é linear, onde os coeficientes das variáveis de projeto $d_{i}$ são as derivadas (ou sensibilidades) da função objetivo do problema de otimização (função $F$ ) em relação à variável do problema.

O mesmo cálculo deve ser feito com as restrições, o que resulta nas seguintes equações:

$$
\begin{aligned}
& g_{1}^{\text {linear }}=\frac{\partial g_{1}}{\partial d_{1}} d_{1}+\cdots+\frac{\partial g_{1}}{\partial d_{n}} d_{n} \leq c-\left(g_{0_{1}}-\frac{\partial g_{1}}{\partial d_{1}} d_{0_{1}}-\cdots-\frac{\partial g_{1}}{\partial d_{n}} d_{0_{n}}\right) \\
& g_{2}^{\text {linear }}=\frac{\partial g_{2}}{\partial d_{2}} d_{1}+\cdots+\frac{\partial g_{2}}{\partial d_{n}} d_{n} \leq c-\left(g_{0_{2}}-\frac{\partial g_{2}}{\partial d_{2}} d_{0_{2}}-\cdots-\frac{\partial g_{2}}{\partial d_{n}} d_{0_{n}}\right) \\
& g_{3}^{\text {linear }}=\frac{\partial g_{3}}{\partial d_{3}} d_{1}+\cdots+\frac{\partial g_{3}}{\partial d_{n}} d_{n} \leq c-\left(g_{0_{3}}-\frac{\partial g_{3}}{\partial d_{3}} d_{0_{3}}-\cdots-\frac{\partial g_{3}}{\partial d_{n}} d_{0_{n}}\right)
\end{aligned}
$$

A cada iteração são definidos os limites móveis para as variáveis de projeto (Fig. 5.2). Esses limites são definidos como valores em relação ao valor dessa variável. Se os valores utilizados para os limites móveis forem muito grandes, o erro da aproximação linear será grande, podendo causar inclusive a perda do ponto ótimo. Se forem muito pequenos o custo computacional para obter a solução ótima será muito grande. Uma forma de tentar minimizar esse impasse e tornar mais rápido a convergência da solução é assumir valores grandes para os limites móveis na região em que a função objetivo não-linear apresenta uma curvatura menos acentuada (por exemplo, região do ponto $d_{0}$ mostrada na Fig. 5.2).

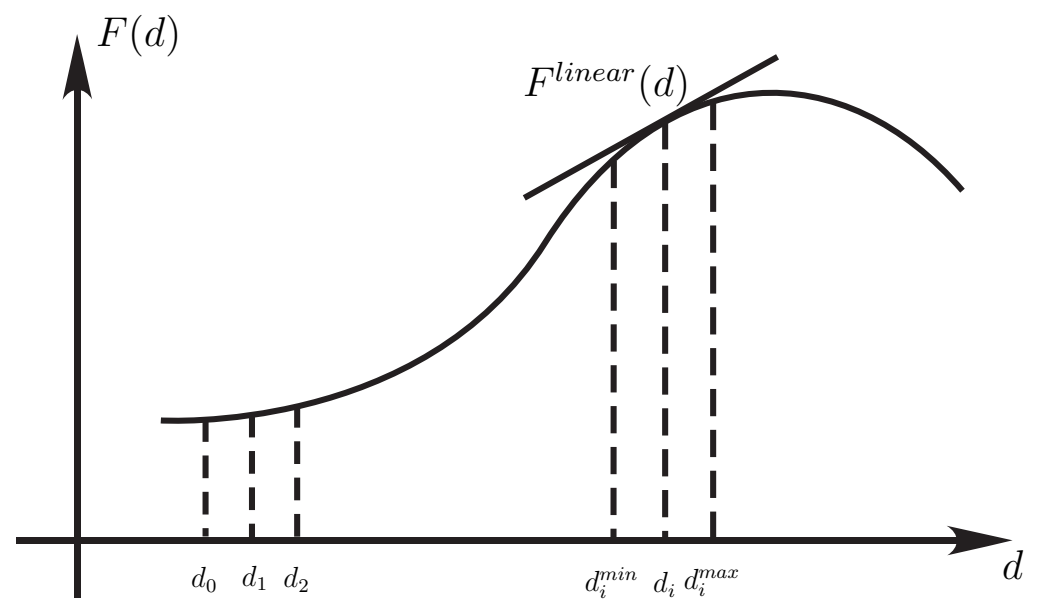

Figura 5.2: Limites móveis

Já na região em que a função apresenta uma curvatura mais acentuada (por exemplo, região do ponto $d_{i}$ mostrada na Fig. 5.2) os limites móveis devem ser pequenos. Além disso, à medida que a convergência da solução (ponto ótimo da função objetivo não-linear) se aproxima, os limites móveis devem ser reduzidos. Assim, uma escolha inadequada dos valores dos limites móveis pode tornar o método PLS desvantajoso. É adotado neste trabalho, como geralmente utilizado na literatura, as variáveis de projeto $\left(d_{i}\right)$ alterarem de 5-15\% de seus valores originais (HAFTKA; GURDAL; KAMAT, 1990), em cada iteração. 
Após a otimização, um novo conjunto de variáveis de projeto $d_{i}$ é obtido e atualizado no modelo de elementos finitos. A iteração continua até que seja atingida a convergência para o valor da função objetivo.

A Fig. 5.3 mostra o diagrama de fluxo de um programa em MatLab para resolver o problema de MOT proposto. Os dados iniciais são fornecidos ao programa com informação sobre a malha de MEF do domínio, incluindo as variáveis de projeto iniciais $d_{n 0}$. É feito o cálculo das pseudo-densidades $\gamma_{n}$ em função das variáveis de projeto $d_{n}$ utilizando a função de projeção. Em seguida, o cálculo do MEF harmônico é feito em paralelo com o MEF modal e o MAC. Calculam-se as funções objetivos e restrições. Então, é verificada a convergência da função objetivo, se sim, tem-se o resultado final, se não, as sensibilidades das funções objetivo e restrições são calculadas em relação à variável de projeto $d_{n}$. Essas informações são fornecidas ao algoritmo de otimização que utiliza a PLS para obter uma nova distribuição de material que é atualizada para o cálculo das pseudo-densidades e então, procede-se para uma nova iteração, até a convergência ser alcançada.

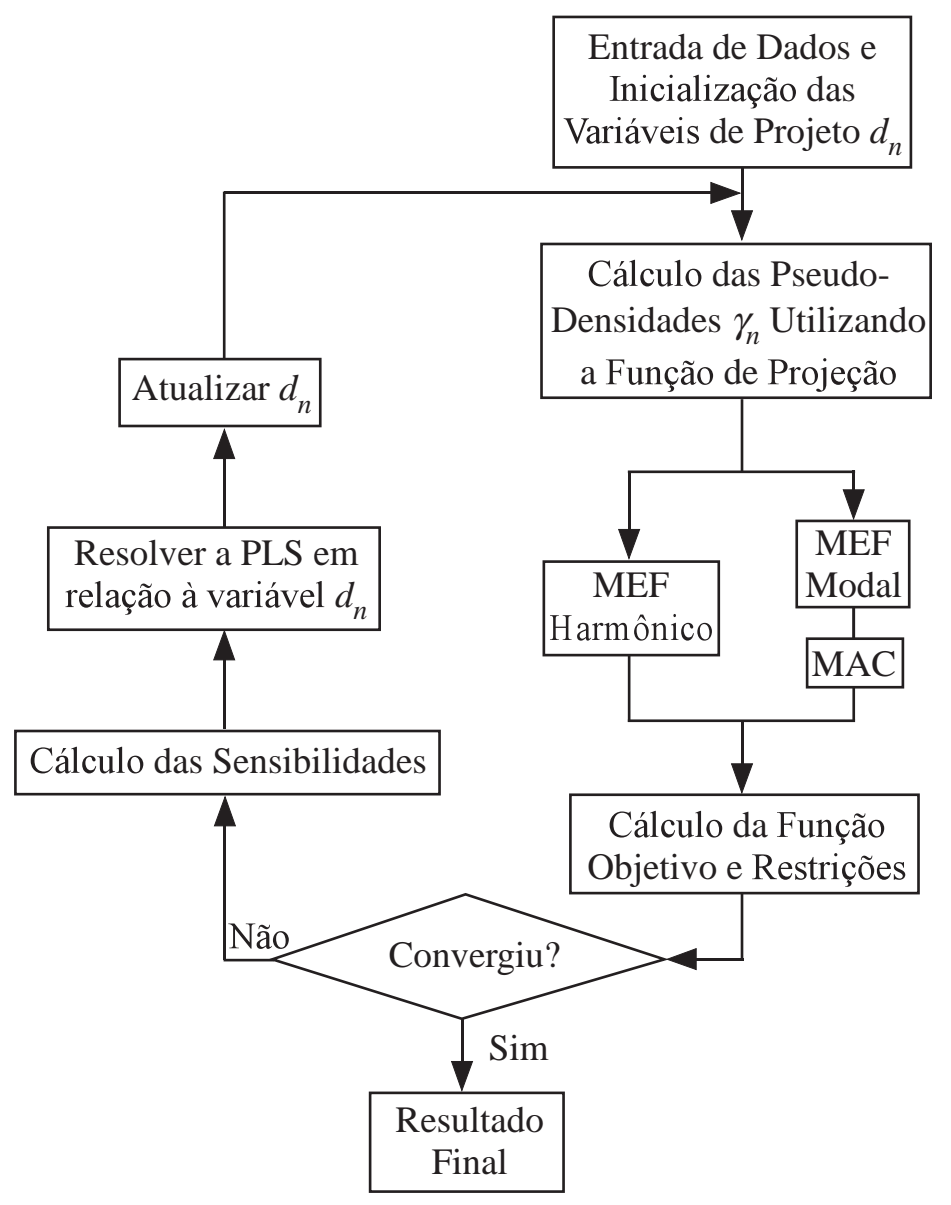

Figura 5.3: Diagrama de fluxo para o Método de Otimização Topológica. 


\section{RESULTADOS}

Neste capítulo são apresentados alguns resultados da otimização aplicada ao sonotrodo do tipo bloco obtidos com o software implementado. Assim, esses resultados verificam se a formulação da função multi-objetivo e a implementação do software podem ser utilizadas para este problema. O modelo tridimensional 3D da estrutura, considerando o transdutor piezelétrico, o amplificador e o sonotrodo, está representado na Fig. 6.1.

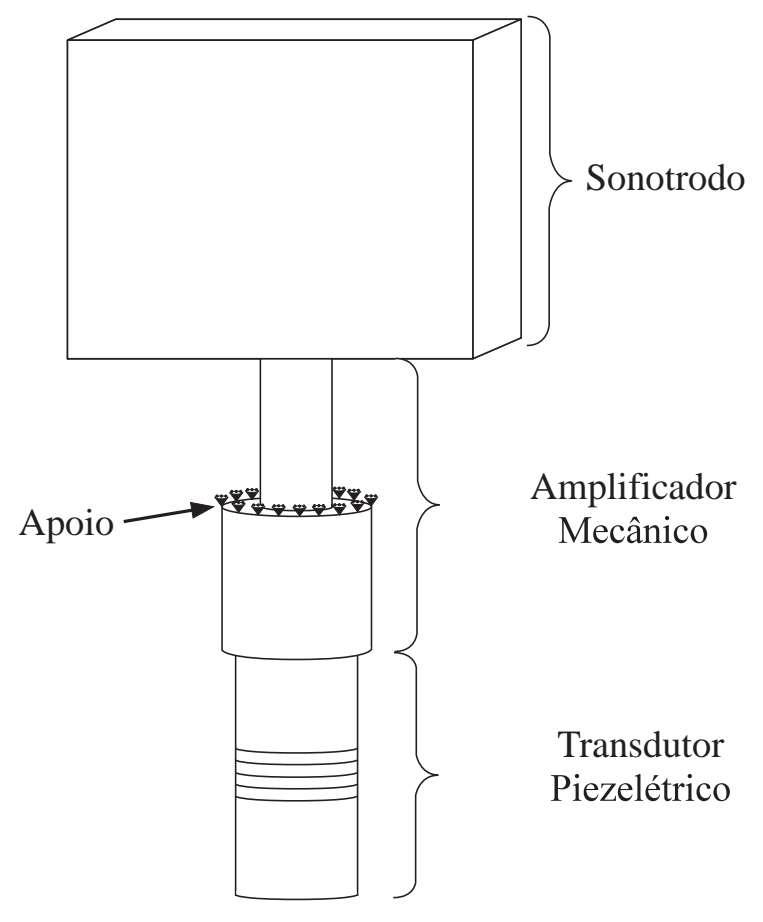

Figura 6.1: Representação do modelo em 3D.

No entanto, para que modelos de MEF 3D apresentem resultados precisos, é necessário um número muito grande de elementos (aproximadamente 100000 elementos para este caso 3D, por exemplo), o que gera um custo computacional muito alto. Para reduzir este custo computacional no processo de otimização topológica, foi considerado um modelo bidimensional 2D, como representado na Fig. 6.2(a), onde tem-se o sonotrodo modelado como elemento plano, e o amplificador mecânico e o transdutor piezelétrico modelados como elementos axissimétricos. Além disso, pode-se também utilizar a simetria da 
estrutura a fim de se reduzir ainda mais o custo computacional, ou seja, para esta estrutura, pode-se utilizar apenas a metade do domínio (Fig. 6.2(b)).

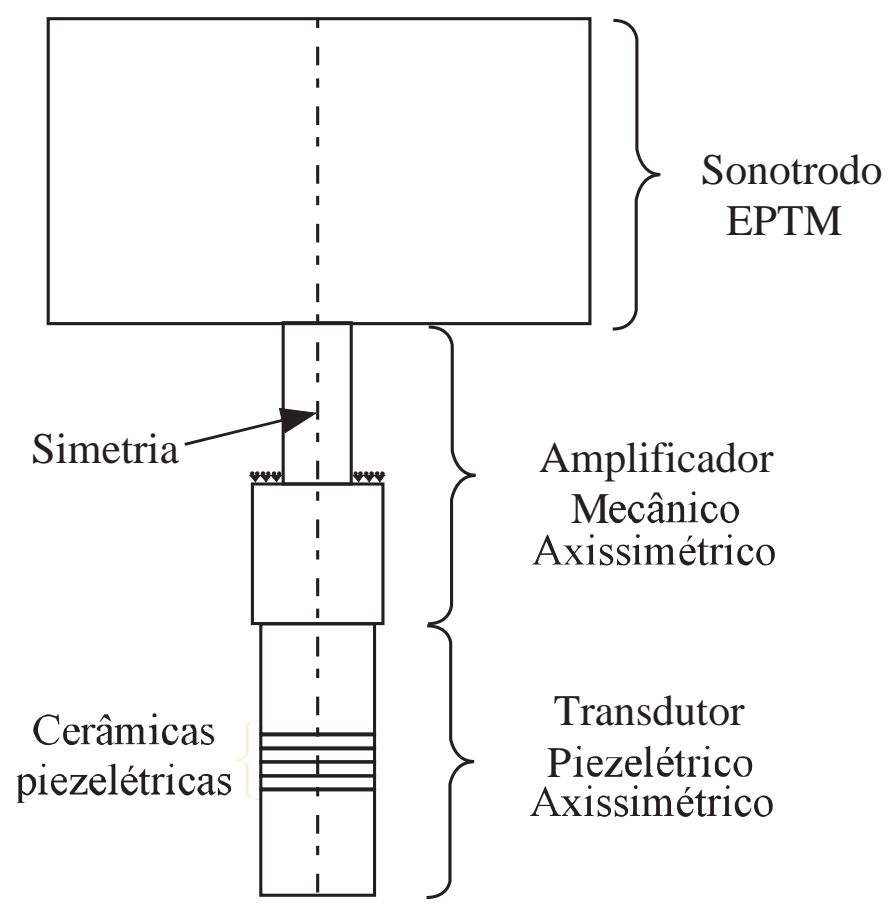

(a)

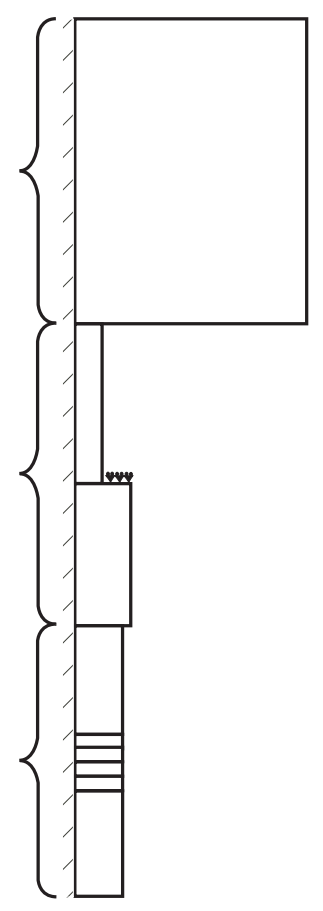

(b)

Figura 6.2: Representação do modelo em 2D, (a) domínio completo e (b) domínio simplificado pela simetria.

\subsection{Materiais utilizados}

Os materiais utilizados são:

- Material PZT-8 para as cerâmicas piezelétricas;

- Aço 4340 para o sonotrodo e amplificador;

- Alumínio 7075 para as massas metálicas que constituem o transdutor piezelétrico.

A Tabela 6.1 contém os valores das propriedades piezelétricas do Titanato Zircanato de Chumbo (PZT-8) utilizado na simulação numérica para todos os exemplos presentes. $\mathbf{c}^{E}$, e e $\varepsilon$ são as propriedades elásticas, piezelétricas e dielétricas, respectivamente, do meio piezelétrico, e $\rho$ é a densidade do material piezelétrico. A Tabela 6.2 contém os valores do módulo de elasticidade, da densidade e do módulo de Poisson do aço 4340 e do alumínio 7075. 
Tabela 6.1: Propriedades do material PZT-8.

\begin{tabular}{lr|lr}
\hline$c_{11}^{E}\left(10^{10} \mathrm{~N} / \mathrm{m}^{2}\right)$ & 13,7 & $e_{31}\left(C / \mathrm{m}^{2}\right)$ & $-4,0$ \\
$c_{12}^{E}\left(10^{10} \mathrm{~N} / \mathrm{m}^{2}\right)$ & 6,97 & $e_{33}\left(C / \mathrm{m}^{2}\right)$ & 13,8 \\
$c_{13}^{E}\left(10^{10} \mathrm{~N} / \mathrm{m}^{2}\right)$ & 7,16 & $e_{15}\left(C / \mathrm{m}^{2}\right)$ & 10,4 \\
$c_{33}^{E}\left(10^{10} \mathrm{~N} / \mathrm{m}^{2}\right)$ & 12,4 & $\varepsilon_{11}\left(10^{-9} \mathrm{~F} / \mathrm{m}\right)$ & 7,9473 \\
$c_{44}^{E}\left(10^{10} \mathrm{~N} / \mathrm{m}^{2}\right)$ & 3,4 & $\varepsilon_{33}\left(10^{-9} \mathrm{~F} / \mathrm{m}\right)$ & 5,1507 \\
$c_{66}^{E}\left(10^{10} \mathrm{~N} / \mathrm{m}^{2}\right)$ & 3,365 & $\rho\left(\mathrm{kg} / \mathrm{m}^{3}\right)$ & 7700 \\
\hline
\end{tabular}

Tabela 6.2: Propriedades do Aço 4340 e do Alumínio 7075.

\begin{tabular}{ccc}
\hline Propriedades & Aço 4340 & Alumínio 7075 \\
\hline$E_{0}(G P a)$ & 208,3 & 74,32 \\
$\rho\left(k g / m^{3}\right)$ & 7846,3 & 2901,3 \\
$\nu$ & 0,29 & 0,34 \\
\hline
\end{tabular}

O material PZT-8 é utilizado como material piezelétrico neste trabalho porque ele é o mais adequado para utilização em transdutores de potência, pois possui baixas perdas mecânicas, alta resistência mecânica, alta resistência à despolarização sob tensão mecânica, alta temperatura de Curie $\left(390^{\circ} \mathrm{C}\right)$, baixas perdas dielétricas sob altos regimes de excitação elétrica e alto coeficiente de acoplamento mecânico (SILVA, 2006).

Para o sonotrodo e o amplificador mecânico, o material utilizado é o Aço 4340 por possuir baixas perdas mecânicas, alto módulo de elasticidade e alta resistência à fadiga e à corrosão.

Para as massas metálicas do transdutor, utiliza-se o Alumínio 7570, pois além de ser um material de alta resistência mecânica, é leve, possui baixa impedância acústica, e o principal de tudo: devido ao baixo módulo de elasticidade do alumínio, um transdutor feito com alumínio apresenta uma deformação mais alta, ou seja ele vibra com maior amplitude do que um transdutor feito de aço, por exemplo. Isso é importante em aplicações de alta potência como solda. Outro motivo importante é utilizar o alumínio para aumentar o coeficiente de acoplamento eletromecânico do transutor.

\subsection{Teste de Convergência}

Com o domínio de projeto definido, e os materiais escolhidos, é necessário definir o tamanho de elemento que fornece um resultado preciso. Para isso é feito um teste de convergência onde o mesmo domínio é analisado utilizando-se diferentes tamanhos de 
elementos, cada vez menores, até que a solução entre uma discretização e outra seja menor que um erro pré-determinado.

Contudo, deve-se avaliar também que quanto menor o tamanho do elemento, maior é o custo computacional. Então, o valor do erro deve ser estipulado de modo a avaliar a convergência, levando em consideração o custo computacional.

A Tabela 6.3 a seguir apresenta as dez primeiras freqüências de ressonância para o domínio da Fig. 6.2(b), obtidas para tamanhos de elementos de $5 \mathrm{~mm}$ e $2,5 \mathrm{~mm}$ utilizando o programa desenvolvido neste trabalho, e para tamanho de elemento de $1 \mathrm{~mm}$ utilizando o software comercial ANSYS ${ }^{\circledR}$. A necessidade de se utilizar o software comercial ANSYS ${ }^{\circledR}$ na análise de discretização de $1 \mathrm{~mm}$ decorre da falta de memória acusada pelo MatLab ${ }^{\circledR}$, pois as matrizes se tornaram grandes demais para processamento de autovalores e autovetores pela rotina "eigs" do MatLab ${ }^{\circledR}$.

As colunas referentes às dicretizações de $2,5 \mathrm{~mm}$ e $5 \mathrm{~mm}$ apresentam também, o erro, em \%, em relação à discretização de $1 \mathrm{~mm}$. A última linha da Tabela 6.3 apresenta os tempos de processamento para as discretizações de $5 \mathrm{~mm}$ e 2,5 $\mathrm{mm}$, utilizando uma CPU com processador Intel ${ }^{\circledR}$ Core2Quad com 3GB de memória RAM. O tempo de processamento para a discretização de $1 \mathrm{~mm}$ não foi registrada, pois como já foi dito, a análise foi feita no software comercial ANSYS ${ }^{\circledR}$.

Tabela 6.3: Teste de convergência.

\begin{tabular}{c|c|c|c}
\hline & \multicolumn{3}{|c}{ Freqüência de Ressonância $(H z)$} \\
\hline \multirow{2}{*}{ Modo } & \multicolumn{3}{|c}{ Discretização } \\
& $1 \mathrm{~mm}$ & $2,5 \mathrm{~mm}($ erro \%) & $5 \mathrm{~mm}($ erro \%) \\
\hline 1 & 3140 & $3166,6(0,84)$ & $3203,6(1,98)$ \\
2 & 8285,9 & $8299,4(0,16)$ & $8324,6(0,46)$ \\
3 & 9776,2 & $9791(0,15)$ & $9814,3(0,39)$ \\
4 & 11193 & $11200(0,06)$ & $11213(0,18)$ \\
5 & 17523 & $17261(1,51)$ & $17287(1,36)$ \\
6 & 17905 & $17929(0,13)$ & $17974(0,38)$ \\
7 & 20707 & $20720(0,06)$ & $20748(0,19)$ \\
8 & 23688 & $23732(0,18)$ & $23828(0,59)$ \\
9 & 24191 & $24282(0,37)$ & $24438(1,01)$ \\
10 & 27311 & $27398(0,31)$ & $27527(0,78)$ \\
\hline Tempo de pro- & - & 21,45 & 2,44 \\
cessamento $(s)$ & & \multicolumn{2}{|c}{} \\
\hline
\end{tabular}

Então, analisando a Tabela 6.3 decidiu-se por utilizar a discretização com $5 \mathrm{~mm}$ como tamanho de elemento, pois a diferença para os resultados utilizando $1 \mathrm{~mm}$ e 2,5 $\mathrm{mm}$ é 
baixa (inferior à 2\%), e o tempo de processamento é significativamente inferior que na análise utilizando discretização de 2,5 $\mathrm{mm}$ (19,01 $\mathrm{s}$ a menos).

\subsection{Comparação 2D $\backslash 3 \mathrm{D}$}

No entanto, para validar a utilização do domínio plano a fim de se reduzir o custo computacional, é necessário fazer a comparação entre os resultados obtidos para os casos 2D e 3D. Para a análise 2D foi utilizado o domínio da Fig. 6.2(b), e para a análise 3D o domínio da Fig. 6.3 por exemplo, que utiliza a simetria em dois planos para analisar apenas 1/4 da estrutura. Para a análise 2D é possível utilizar elementos quadrados pela simplicidade da geometria, mas para a análise 3D é necessário utilizar elementos tetraédricos por causa das geometrias cilíndricas.

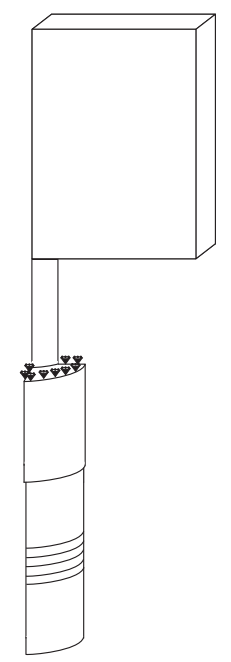

Figura 6.3: Representação da simetria utilizada no modelo 3D.

Como exemplo, foi feita a comparação do segundo (Fig. 6.4), quinto (Fig. 6.5) e sétimo (Fig. 6.6) modos de vibração e suas respectivas freqüências de ressonância. Para o caso 3D são apresentadas a vista frontal e isométrica.

Como pode ser observado, os modos de vibração para a análise em 2D apresentados correspondem ao mesmo modo de vibrar da análise em 3D, e as freqüências de ressonância obtidas na análise em 2D diferem daquelas obtidas na análise em 3D em 2, 98\%, 0,37\% e 0,76\% para o segundo, quinto e sétimo modos, respectivamente.

A Tabela 6.4 apresenta as dez primeiras freqüências de ressonância das análises 2D e 3D e os respectivos erros. Como estamos procurando o modo de vibração próximo de $20 k H z$, a grande diferença apresentada no primeiro modo não influi no resultado final. 


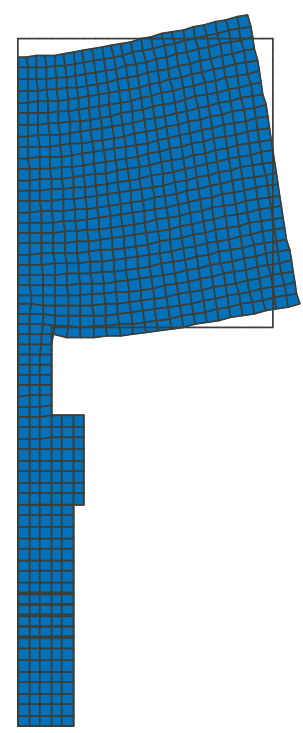

(a)

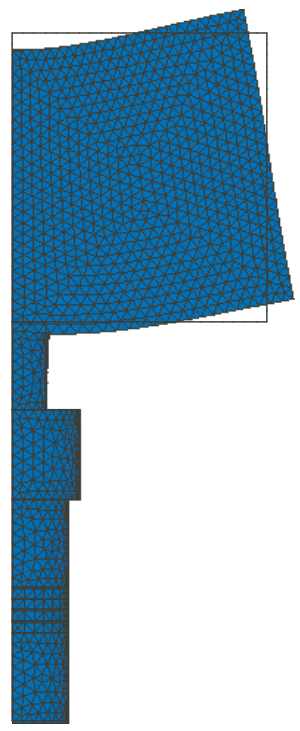

(b)

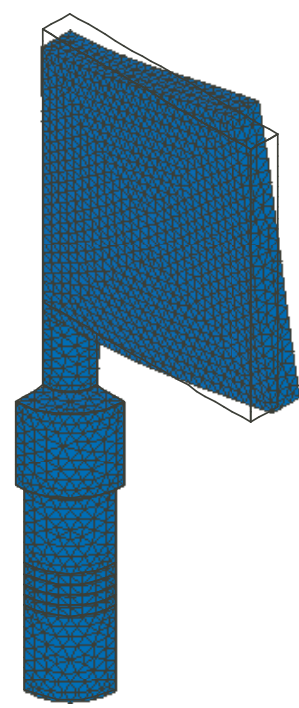

(c)

Figura 6.4: Comparação do segundo modo de vibração. Análise 2D (a) com a freqüência de ressonância em 8324,6 Hz, e análise 3D, (b) vista frontal e (c) vista isométrica, com a freqüência de ressonância em $8076,6 \mathrm{~Hz}$.

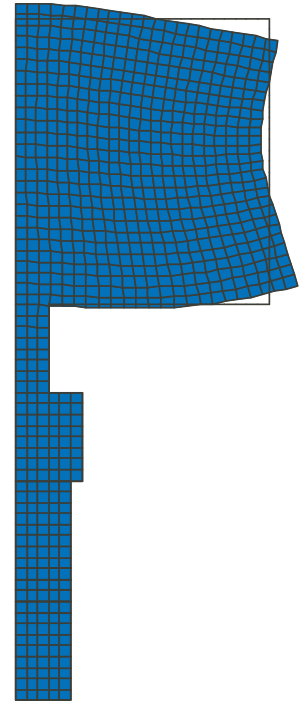

(a)

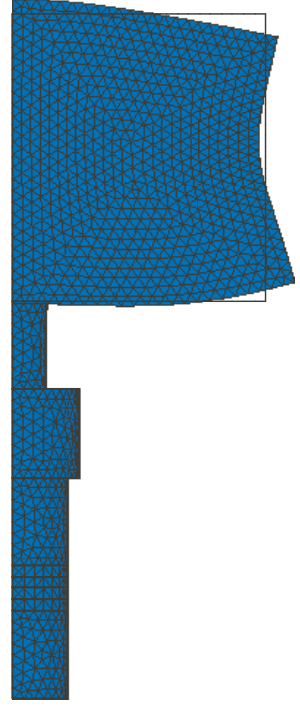

(b)

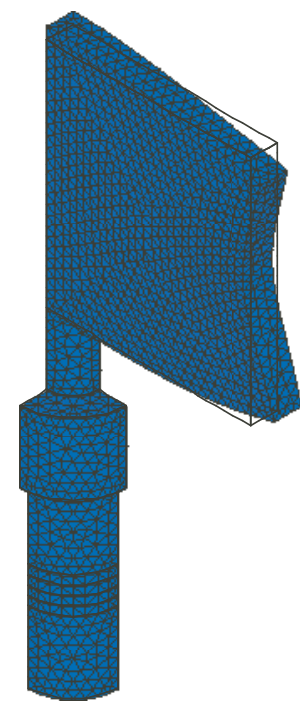

(c)

Figura 6.5: Comparação do quinto modo de vibração. Análise 2D (a) com a freqüência de ressonância em $17287 \mathrm{~Hz}$, e análise 3D, (b) vista frontal e (c) vista isométrica, com a freqüência de ressonância em $17223 \mathrm{~Hz}$.

Portanto, mediante a comparação feita apresentada nas Figs. 6.4, 6.5, 6.6 e na Tabela 6.4, este trabalho foi realizado utilizando um modelo 2D do domínio de projeto.

No entanto, deve-se considerar que, ao utilizar o domínio em 2D, aproxima-se o 


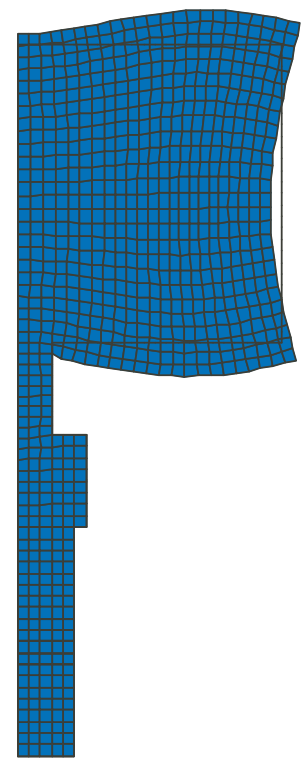

(a)

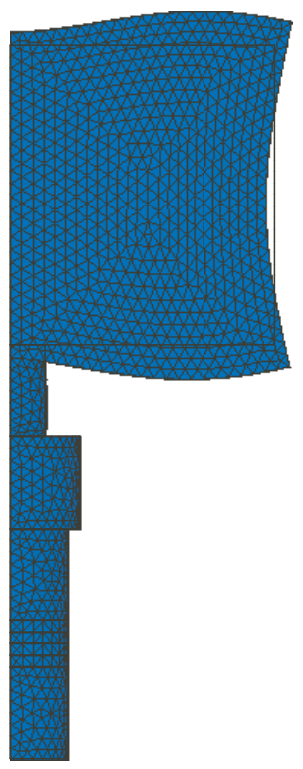

(b)

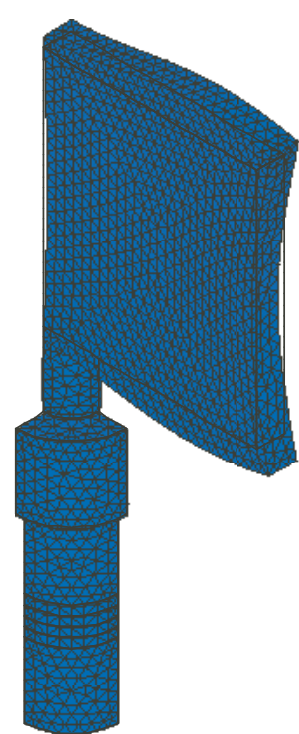

(c)

Figura 6.6: Comparação do sétimo modo de vibração. Análise 2D (a) com a freqüência de ressonância em $20748 \mathrm{~Hz}$, e análise 3D, (b) vista frontal e (c) vista isométrica, com a freqüência de ressonância em $20590 \mathrm{~Hz}$.

Tabela 6.4: Dez primeiras freqüências de ressonância para as análises em 2D e 3D.

\begin{tabular}{c|c|c|c}
\hline Modo & $2 \mathrm{D}(\mathrm{Hz})$ & $3 \mathrm{D}(\mathrm{Hz})$ & Erro $(\%)$ \\
\hline 1 & 3203,6 & 2602,2 & 18,77 \\
2 & 8324,6 & 8076,6 & 2,98 \\
3 & 9814,3 & 9786,9 & 0,28 \\
4 & 11213 & 11117 & 0,86 \\
5 & 17287 & 17223 & 0,37 \\
6 & 17974 & 17693 & 1,56 \\
7 & 20748 & 20590 & 0,76 \\
8 & 23828 & 23563 & 1,11 \\
9 & 24438 & 23956 & 1,97 \\
10 & 27527 & 27366 & 0,58 \\
\hline
\end{tabular}

acoplamento entre o amplificador (elemento axissimétrico) e o sonotrodo (elemento plano), bem como desprezam-se alguns modos em outros planos e modos não simétricos da estrutura, que somente seriam verificados numa análise do modelo em 3D completo. A análise do acoplamento e a análise modal do domínio em 3D completo podem ser verificadas no Anexo B. 


\subsection{Resultados Numéricos Utilizando o Programa Implementado}

Os exemplos apresentados neste capítulo utilizam a formulação descrita no Capítulo 4 e o programa implementado em MatLab ${ }^{\circledR}$. Os resultados são obtidos a partir de diferentes parâmetros iniciais, tais como pesos das funções objetivo $\left(w_{1}, w_{2}, w_{3}\right.$ e $\left.w_{4}\right)$, restrição de volume superior $\left(\Theta_{\text {lim_sup }}\right)$, valor inicial para as variáveis de projeto $\left(d_{0}\right)$, o parâmetro de uniformização de deslocamentos $(\beta)$, e o raio do filtro $\left(R_{\max }\right)$. A restrição de volume inferior $\left(\Theta_{\text {lim_inf }}\right)$ é estabelecida como sendo igual a $15 \%$ do volume total. Os limites móveis superior e inferior (limitantes da variação das variáveis de projeto) são definidos iguais a $15 \%$ acima e abaixo, respectivamente, do valor da variável. Para todos os exemplos apresentados, a tensão de excitação é igual a $500 \mathrm{~V}$, e o problema de otimização inicia-se dentro do domínio viável com todas as restrições satisfeitas. Eventualmente, alguns parâmetros podem ser alterados durante a otimização de modo a melhorar o resultado final. Como exemplo, técnica de projeção pode ser desligada nas últimas 10 ou 20 iterações para tentar remover um pouco da escala de cinza.

Então, recapitulando, o objetivo da otimização, neste trabalho, é uniformizar e maximizar os deslocamentos da face de trabalho do sonotrodo (que no caso é a face superior do domínio), maximizar a rigidez da estrutura e garantir que a freqüência do modo de pistão seja maior que $20 \mathrm{kHz}$, que é o limite da freqüência audível. Pelo mesmo motivo, a freqüência de excitação deve ser estabelecida em um valor acima ou igual a $20 \mathrm{kHz}$. A uniformização é aceita se a razão $U_{\min } / U_{\max }$ for maior que $80 \%$.

Para os exemplos apresentados neste trabalho, é utilizado o domínio apresentado na Fig. 6.7(a), que contém as dimensões, condições de contorno mecânicas e domínio não-otimizável. Na Fig. 6.7(b) são apresentadas as condições de contorno mecânicas e elétricas, bem como os carregamentos elétricos e os carregamentos mecânicos que indicam a região e a direção de otimização. Nas figuras do domínio de projeto estão detalhados o domínio piezelétrico (representado pela cor cinza claro), o domínio axissimétrico não-otimizável englobando o amplificador mecânico e as partes em alumínio do transdutor (na cor cinza escuro), o domínio otimizável (em branco) e o não-otimizável (na cor preta). As regiões não-otimizáveis garantem a presença de material nas regiões onde existe contato com outras partes do domínio e nas regiões de trabalho do sonotrodo. Elas também podem ser utilizadas para restringir o espaço de soluções evitando os múltiplos-mínimos locais existentes em problemas dinâmicos.

Inicialmente o domínio é discretizado com 770 elementos para verificar a formulação 


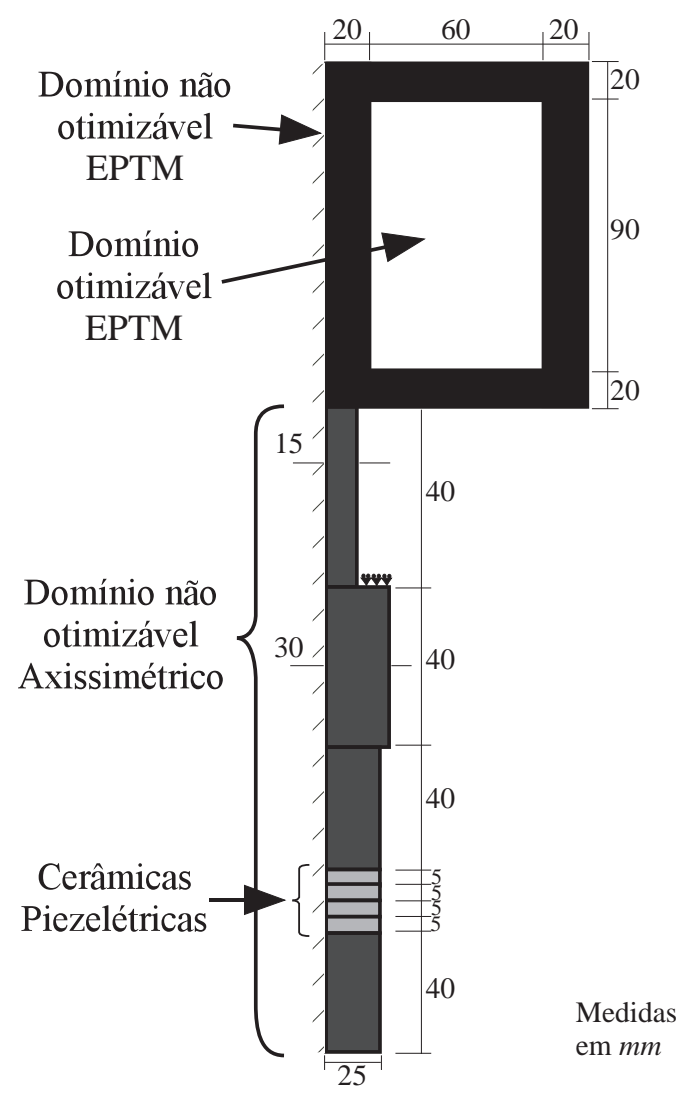

(a)

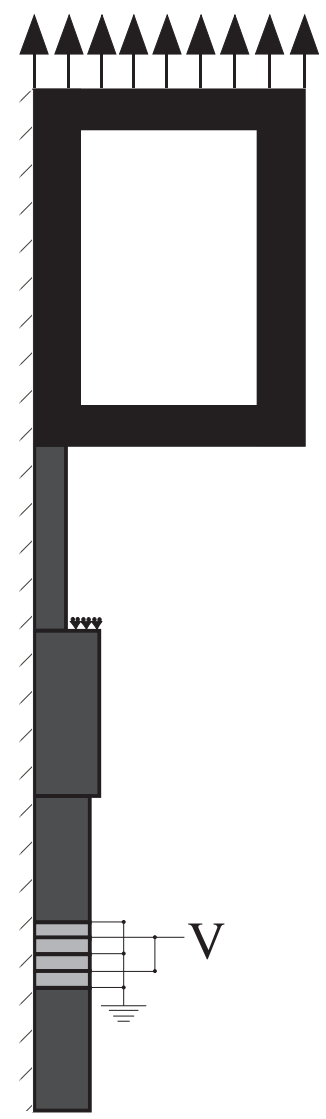

(b)

Figura 6.7: (a) Domínio de projeto. (b) Condiçoes de contorno.

e analisar a influência dos parâmetros, e então, passamos a utilizar 2768 elementos para se obter resultados mais refinados. Para o exemplo de verificação, a otimização termina quando completadas 350 iterações, e para todos os exemplos da análise de influência dos parâmetros, a otimização termina quando completadas 300 iterações. A penalização da densidade $(q)$ no modelo de material inicia com o valor 2 , e aumenta em 2 unidades nas iterações 60, 120, 180 e 240, e a penalização do módulo de elasticidade $(p)$ inicia com o valor 1, e aumenta em meia unidade (0,5) nas iterações 30, 60, 90, 120, 150, 180, 210 e 240. Ao final da otimização, $q=10$ e $p=5$. Geralmente, o método da continuação utiliza a convergência da função para aumentar os valores das penalidades. No entanto, devido à alta sensibilidade da função objetivo pelo fato de se trabalhar com o comportamento dinâmico da estrutura, a convergência só é obtida diminuindo-se os limites móveis das variáveis de projeto, o que aumentaria muito o número de iterações. Por isso foi necessário fixar a mudança das penalizações nas iterações descritas.

Então, neste capítulo, são abordados exemplos que verificam a eficiência e generalidade 
da formulação proposta, a influência dos pesos de cada função objetivo $\left(w_{1}, w_{2}, w_{3}\right.$ e $\left.w_{4}\right)$, do valor inicial das variáveis $\left(d_{0}\right)$, da restrição de volume superior $\left(\Theta_{\text {lim_sup }}\right)$, do parâmetro de uniformização $\beta$ e do raio do filtro $\left(R_{\max }\right)$.

\subsubsection{Verificação da Formulação e do Programa Implementado}

O exemplo apresentado nesta seção utiliza a formulação proposta no Capítulo 4 e o programa implementado em MatLab ${ }^{\circledR}$ para obter um resultado que atenda aos requisitos de projeto. O resultado é obtido utilizando valores de $w_{1}, w_{2}, w_{3}$ e $w_{4}$ iguais a 300,40 , 450 , e 280 respectivamente. Os valores de $d_{0}, \Theta_{\text {lim_sup }}, \beta$ e $R_{\text {max }}$ são iguais a $0,45,55 \%$, 0,027 $\mu \mathrm{m}$ e 5,5 mm, respectivamente. A freqüência de excitação é igual a $20 \mathrm{kHz}$ e a freqüência alvo ( $f_{0}$ da Eq. (4.7) do capítulo de formulação) dessa função é igual a $24 \mathrm{kHz}$, pois a freqüência do modo de pistão deve estar acima de $20 \mathrm{kHz}$. Para essa função, é também necessário determinar o modo desejado a ser procurado, que neste trabalho, é o modo de pistão, apresentado na Fig. 6.8. Assim, a Fig. 6.9 apresenta a topologia ótima obtida pelo MOT, sua deformada (enfatizando o sonotrodo, omitindo a parte do transdutor e amplificador) e o seu modo de pistão.

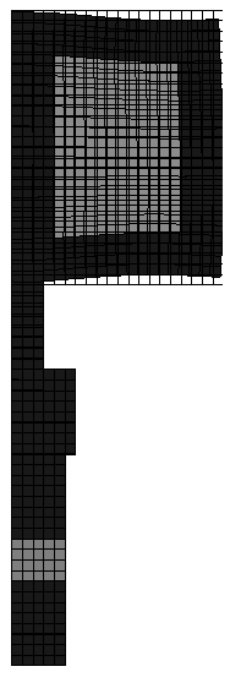

Figura 6.8: Modo de pistão da estrutura da Fig. 6.7(a). Modo alvo a ser seguido durante a otimização.

Na Fig. 6.10 são apresentados os gráficos da função multi-objetivo, $L_{1}, L_{2}, L_{3}$ e $L_{4}$ para mostrar que a convergência está sendo alcançada, ou seja, foram minimizadas as funções multi-objetivo e $L_{1}$, e foram maximizadas as funções $L_{2}$ e $L_{4}$, apesar das grandes oscilações nas curvas de convergência ocasionadas pela alta sensibilidade das funções dinâmicas $L_{1}$, 


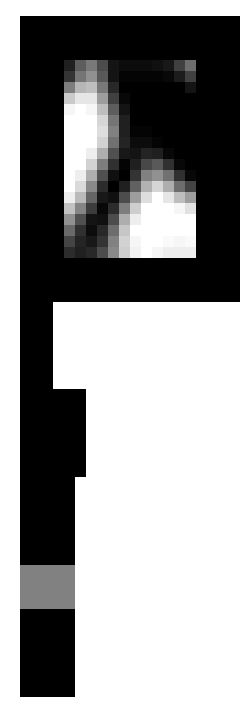

(a) Topologia

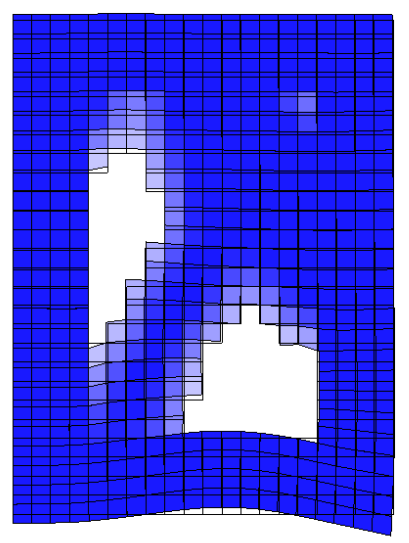

(b) Deformada

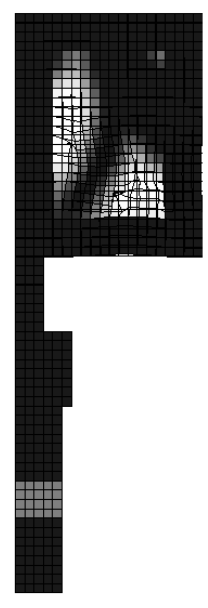

(c) Modo

Figura 6.9: (a) Topologia ótima, (b) deformada (enfatizando o sonotrodo) da topologia ótima a $20 \mathrm{kHz}$, e (c) modo de pistão obtidos com $w_{1}=300, w_{2}=40, w_{3}=450$, $w_{4}=280, d_{0}=0,45, \Theta_{\text {lim_sup }}=55 \%, \beta=0,027 \mu \mathrm{m}$ e $R_{\max }=5,5 \mathrm{~mm}$.

$L_{2}$ e $L_{4}$, em relação às variáveis de projeto. As sensibilidades das funções dinâmicas dependem das derivadas dos seus respectivos vetores de deslocamentos, que por sua vez, dependem das derivadas das matrizes de massa e rigidez da estrutura e da freqüência de excitação, como mostrado na seção 4.8 de sensibilidades. Ou seja, a sensibilidade do problema aumenta ao aumentarmos a freqüência de operação. Já para o caso da função $L_{3}$, embora ela tenha sido maximizada, observa-se que nos intervalos de picos causados pela mudança nas penalizações do modelo de material, a função é minimizada.

Para melhorar a convergência da formulação, os limites móveis (que são utilizados para limitar a variação das variáveis de projeto) são reduzidos se a uniformização $\left(U_{\min } / U_{\max }\right)$ atingir valores maiores que um certo valor. Neste exemplo, inicia-se com os valores dos limites móveis iguais a 15\%. Acima de 200 iterações, se a uniformização atingir um valor maior que $70 \%$, os limites móveis são reduzidos para 10\%, e acima de 250 iterações, o valor dos limites móveis são reduzidos para $5 \%$ se a uniformização ultrapassar $80 \%$.

Para mostrar a uniformização obtida, são apresentados os gráficos da configuração de deslocamentos na face superior do sonotrodo (Fig. 6.11(a)) e o gráfico de uniformização (Fig. 6.11(b)). O gráfico de convergência da freqüência do modo de pistão é apresentado na Fig. 6.11(c). 


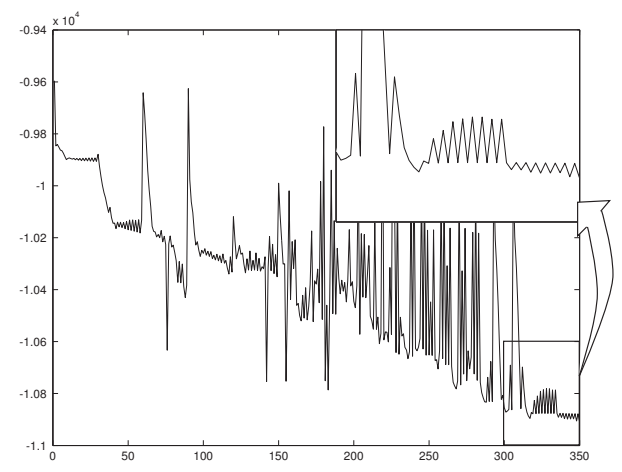

(a) Função multi-objetivo

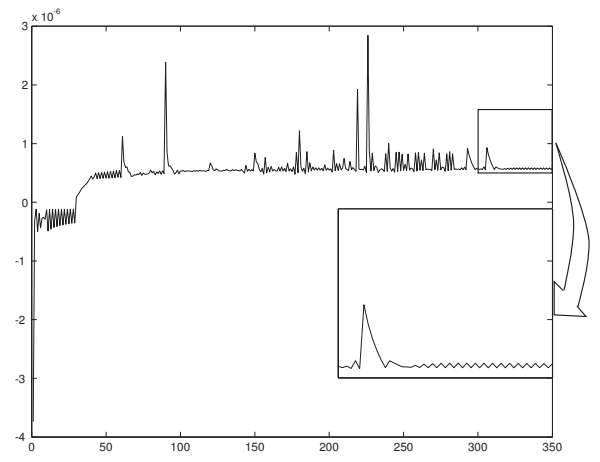

(c) Função $L_{2}$

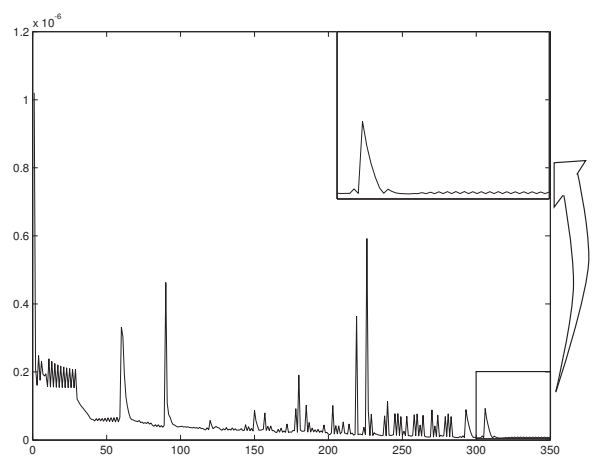

(b) Função $L_{1}$

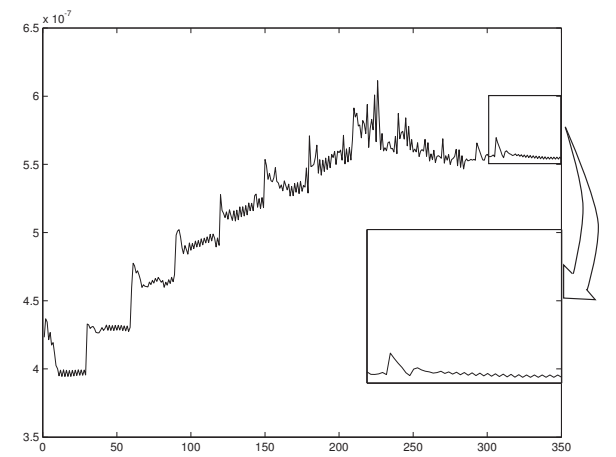

(d) Função $L_{3}$

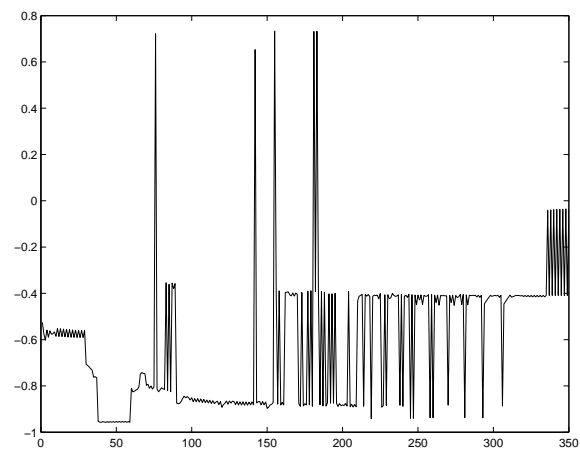

(e) Função $L_{4}$

Figura 6.10: Gráfico de convergência das funções com a vista ampliada das 50 últimas iterações. A abscissa dos gráficos representam o número de iterações e a ordenada os valores das respectivas funções.

Ao final da otimização, a razão de uniformização $U_{\min } / U_{\max }$ e a freqüência do modo de pistão resultaram em valores iguais a 85,43\% e $21119 \mathrm{~Hz}$, respectivamente.

Portanto, a formulação apresentada neste trabalho pode ser utilizada para os ojetivos propostos. Resultados melhores são obtidos modificando os parâmetros iniciais, cujas 


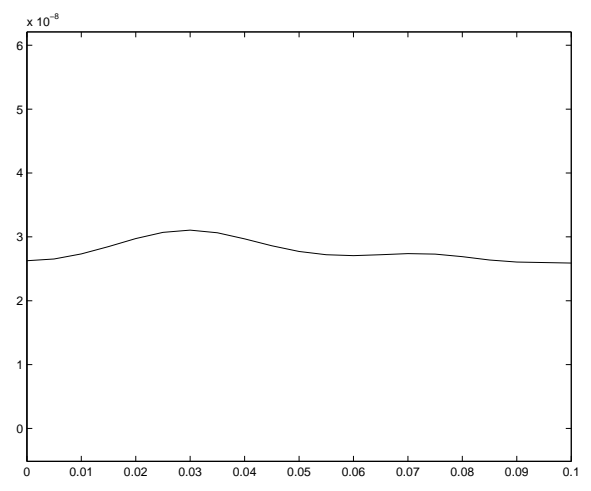

(a) Configuração dos deslocamentos

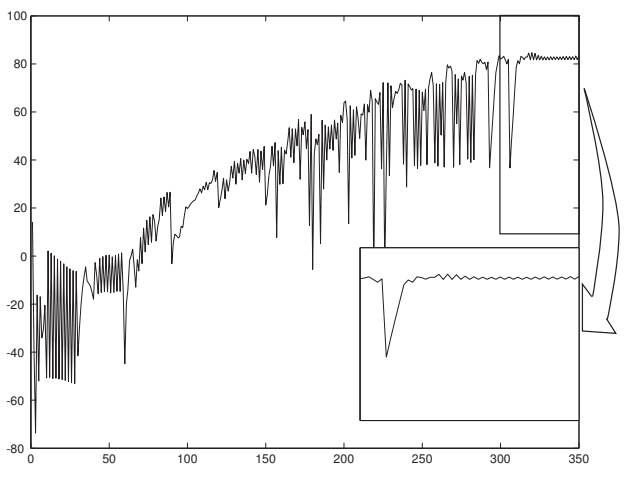

(b) Uniformização $\left(U_{\min } / U_{\max }\right)$

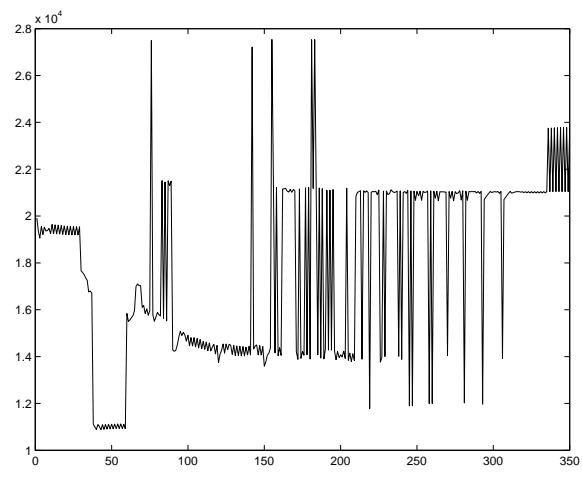

(c) Freqüência do modo de pistão

Figura 6.11: Gráficos de uniformização (com vista ampliada nas últimas 50 iterações) e de freqüência. A abscissa dos gráficos representam o número de iterações e a ordenada os valores das respectivas funções.

influências são estudadas a seguir.

No Anexo A, são apresentados exemplos verificando a generalidade da formulação proposta utilizando-se diferentes freqüências de excitação, como por exemplo, 3 e $10 \mathrm{kHz}$.

\subsubsection{Influência do Coeficiente $w_{1}$ e do Parâmetro de Uniformização $\beta$}

O coeficiente de peso $w_{1}$ permite variar a prioridade da função multi-objetivo em relação à uniformização dos deslocamentos na face de trabalho do sonotrodo. Já o parâmetro $\beta$ pode ser definido como um alvo que esses deslocamentos devem alcançar para a uniformização ser maximizada.

Primeiramente, variando $w_{1}$, obtém-se diferentes resultados do MOT, como mostrados 
na Fig. 6.12, considerando os valores de $w_{1}$ iguais a 200, 400 e 600, respectivamente. Os demais pesos $w_{2}, w_{3}$ e $w_{4}$ são iguais à 20,450, 280 respectivamente, e os valores de $d_{0}$, $\Theta_{\text {lim_sup }}, \beta$ e $R_{\max }$ são iguais a $0,45,47 \%, 0,027 \mu \mathrm{m}$ e $5,5 \mathrm{~mm}$, respectivamente.

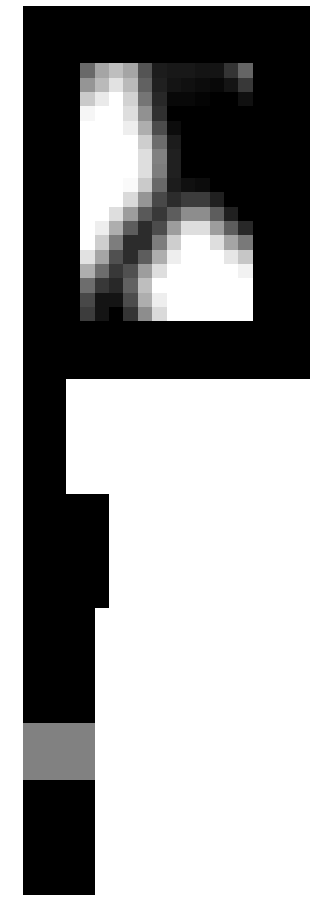

(a) $w_{1}=200$

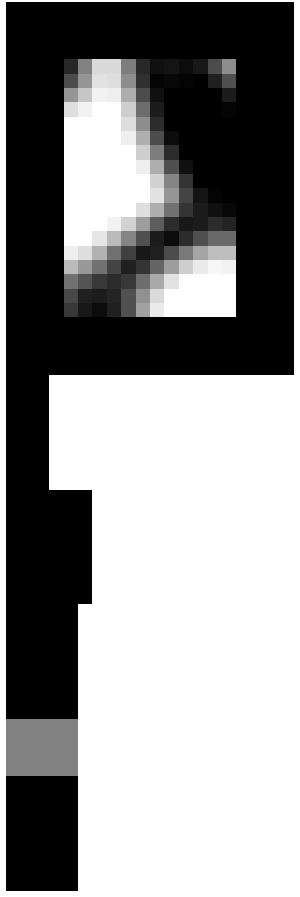

(b) $w_{1}=400$

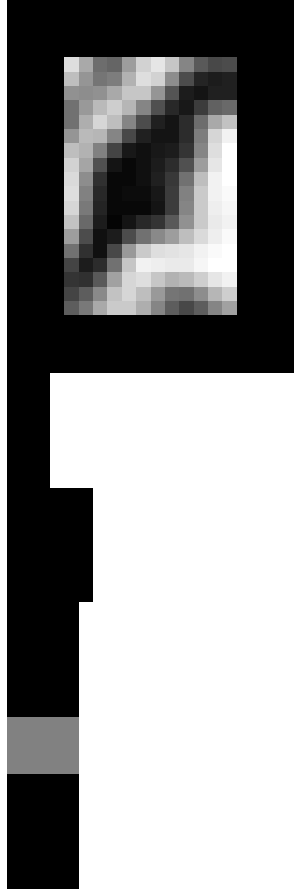

(c) $w_{1}=600$

Figura 6.12: Topologias ótimas para $w_{2}=20, w_{3}=450, w_{4}=280, d_{0}=0,45$, $\Theta_{\text {lim_sup }}=47 \%, \beta=0,027 \mu \mathrm{m}$ e $R_{\max }=5,5 \mathrm{~mm}$.

Os gráficos de convergência da função $L_{1}$ são apresentadas na Fig. 6.13 e mostram que ela foi minimizada. A razão entre as amplitudes mínima e máxima, $U_{\min } / U_{\max }$, que fornece o grau de uniformização das amplitudes, é apresentada na Fig. 6.14 e a configuração dos deslocamentos na face superior do sonotrodo é apresentada na Fig. 6.15.

Ao final da otimização, os casos (a), (b) e (c) resultaram em uma uniformização de 48, 25\%, 59, 75\% e -15, 18\%, respectivamente. Então, analisando também os gráficos das Figs. 6.15 e 6.14 observa-se um aumento na uniformização dos deslocamentos aumentando $w_{1}$ de 200 para 400, contudo, ao aumentar para 600 o valor de $w_{1}$, o resultado esperado seria que a uniformização melhorasse ainda mais, mas o que ocorre é uma variação muito grande na razão $U_{\min } / U_{\max }$ (Fig. 6.14(c)). Isso acontece pois a função $L_{1}$, que é dinâmica, é muito mais sensível às mudanças das variáveis de projeto do que a função $L_{3}$ por exemplo, que é estática, e fazendo $w_{1}=600$, a função multi-objetivo passa a priorizar a função $L_{1}$ (pois $w_{3}$ no caso é igual a 450), dificultando assim, a convergência das funções. Portanto, conclui-se que existe uma relação de interdependência entre as funções $L_{1}$ e $L_{3}$, sendo que 


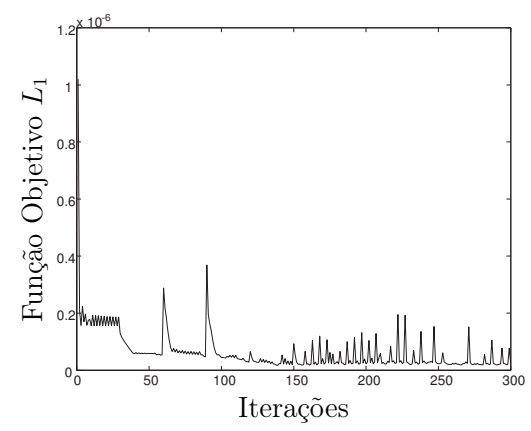

(a) $w_{1}=200$

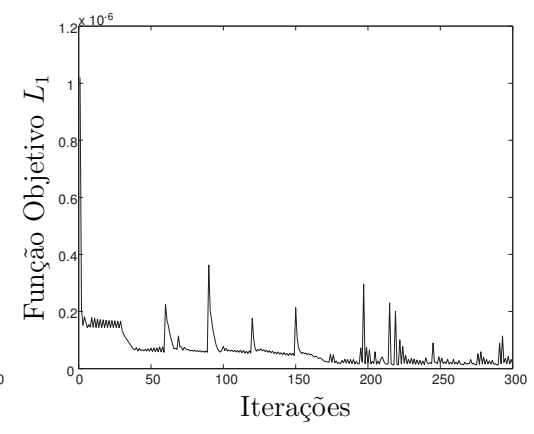

(b) $w_{1}=400$

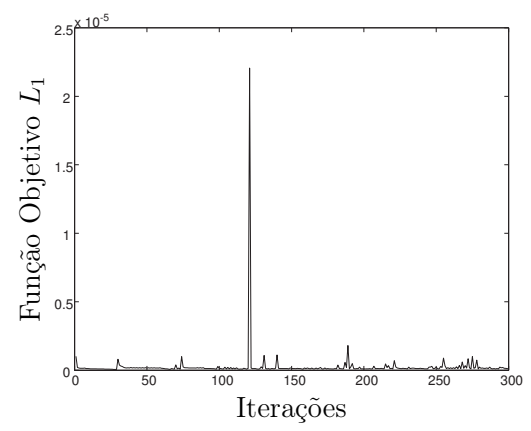

(c) $w_{1}=600$

Figura 6.13: Gráficos de convergência da função $L_{1}$.

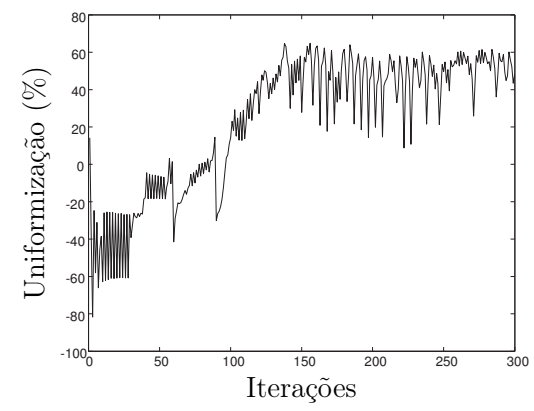

(a) $w_{1}=200$

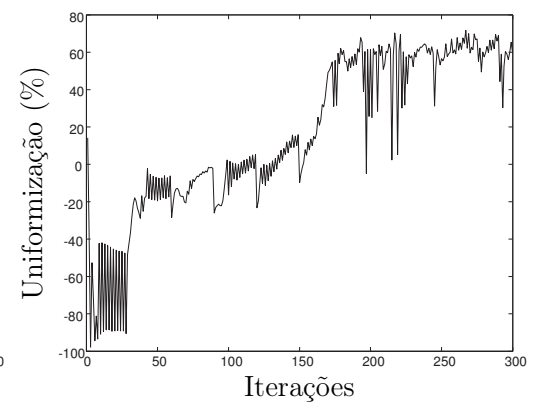

(b) $w_{1}=400$

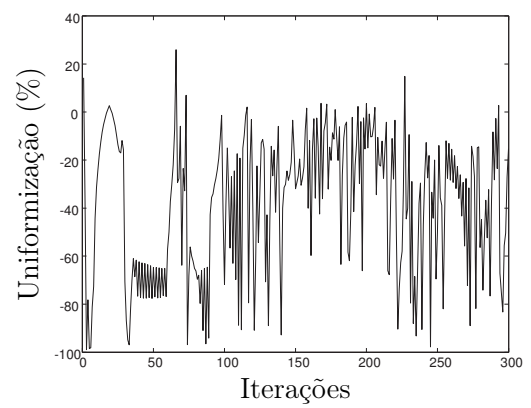

(c) $w_{1}=600$

Figura 6.14: Gráficos da uniformização, razão $U_{\min } / U_{\max }$.

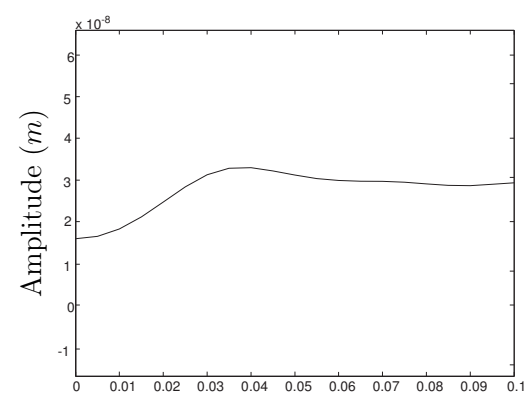

(a) $w_{1}=200$

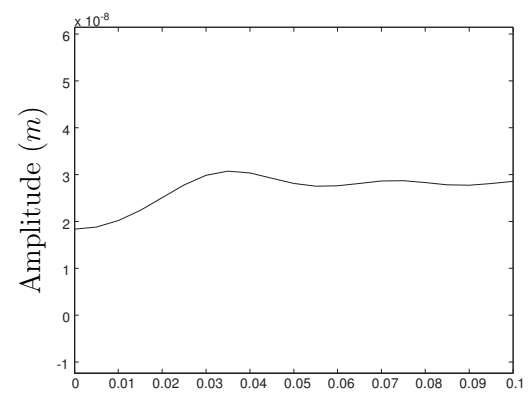

(b) $w_{1}=400$

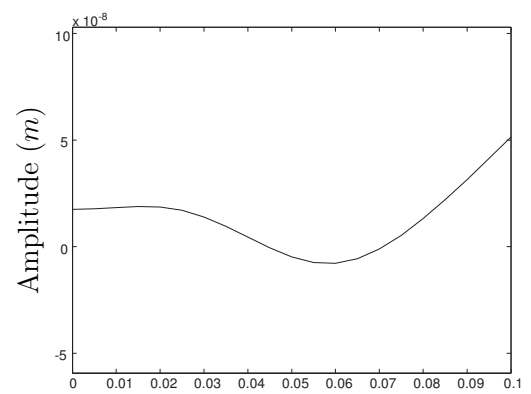

(c) $w_{1}=600$

Figura 6.15: Configuração dos deslocamentos na face de trabalho do sonotrodo. A abscissa representa o comprimento do sonotrodo em $m$.

a primeira deve ser menor que a segunda.

A influência da variação de $\beta$ nos resultados pode ser observado nas figuras a seguir, utilizando valores iguais a 0,025 $\mu \mathrm{m}, 0,027 \mu \mathrm{m}$ e 0,03 $\mu \mathrm{m}$ para os casos (a), (b) e (c), respectivamente. Os demais parâmetros são: $w_{1}=300, w_{2}=50, w_{3}=450, w_{4}=280$, 
$d_{0}=0,45, \Theta_{\text {lim_sup }}=47 \%$, e $R_{\max }=5,5 \mathrm{~mm}$.

A Fig. 6.16 mostra as diferentes topologias ótimas obtidas, e os gráficos da Fig. 6.17 apresentam a influência de $\beta$ na uniformização dos deslocamentos ao final da otimização, resultando em 13,99\%, 72,04\% e 64,93\% para os casos (a), (b) e (c), respectivamente. Então, observando as figuras e gráficos apresentados, nota-se que a sensibilidade do MOT é muito alta em relação ao parâmetro $\beta$. Ou seja, modificando apenas 0,002 ou 0,003 $\mu \mathrm{m}$, toda a topologia ou o gráfico de convergência se modifica.

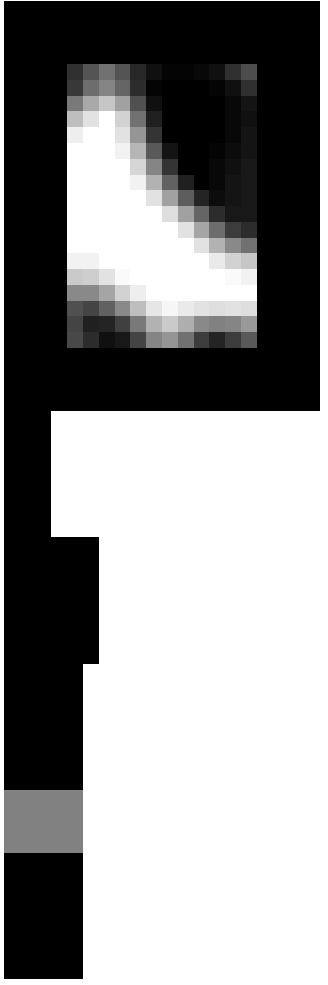

(a) $\beta=0,025 \mu m$

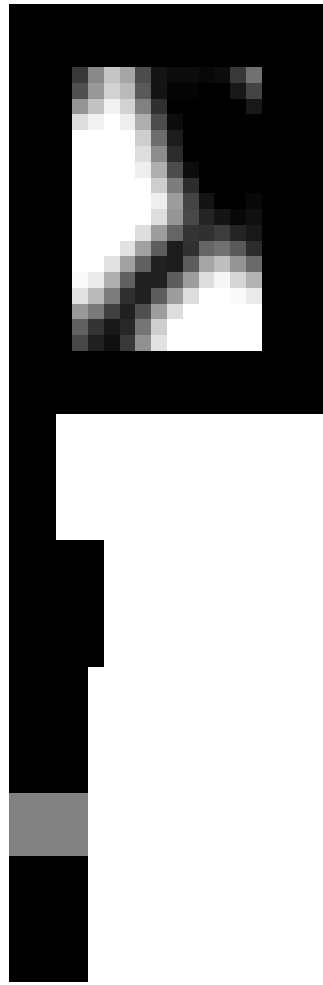

(b) $\beta=0,027 \mu \mathrm{m}$

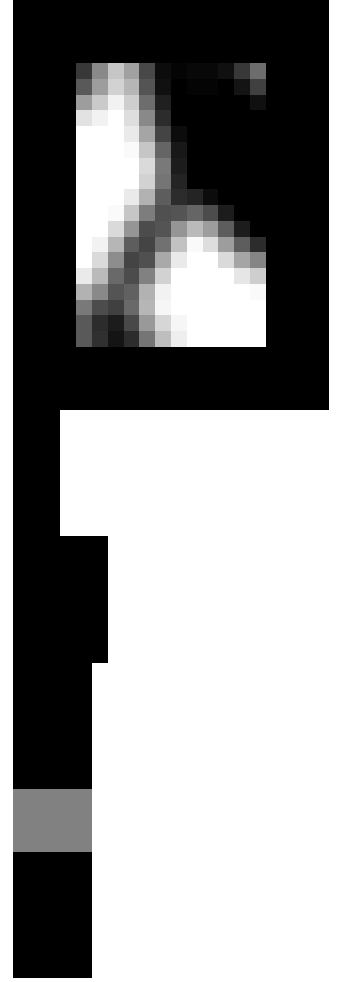

(c) $\beta=0,03 \mu m$

Figura 6.16: Topologias ótimas utilizando diferentes valores para $\beta$.

\subsubsection{Influência do coeficiente $w_{2}$}

O coeficiente de peso $w_{2}$ permite variar a prioridade da função multi-objetivo em relação à maximização dos deslocamentos na face de trabalho do sonotrodo. Portanto, variando $w_{2}$ obtem-se diferentes resultados do MOT, como mostrados na Fig. 6.18, considerando os valores de $w_{2}$ iguais a 20, 50 e 70, respectivamente. Os demais pesos $w_{1}, w_{3}$ e $w_{4}$ são iguais à $300,450,280$ respectivamente, e os valores de $d_{0}, \Theta_{\text {lim_sup }}, \beta$ e $R_{\max }$ são iguais a 0,45,47\%,0,027 $\mu \mathrm{m}$ e 5,5 $\mathrm{mm}$, respectivamente. 


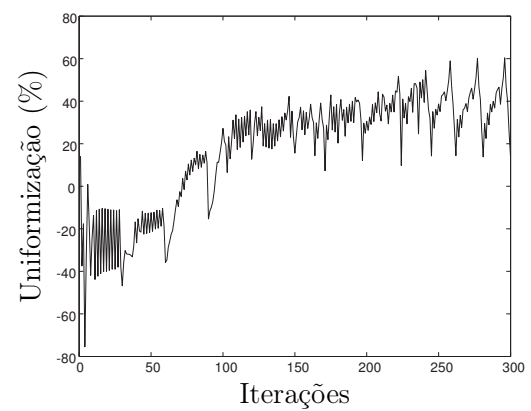

(a) $\beta=0,025 \mu m$

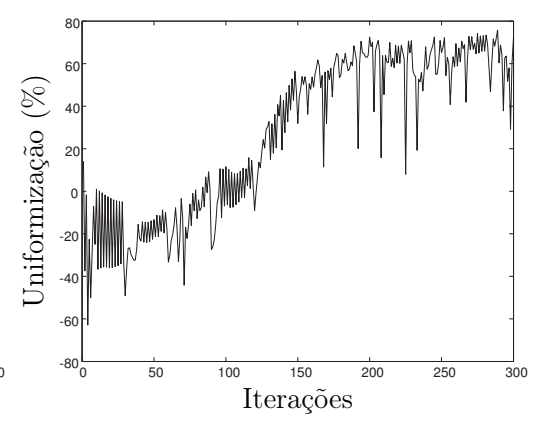

(b) $\beta=0,027 \mu m$

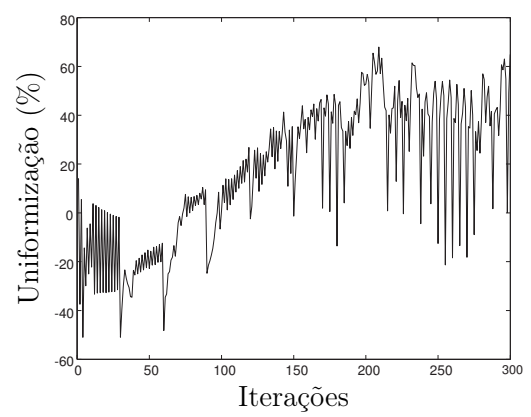

(c) $\beta=0,03 \mu m$

Figura 6.17: Gráficos da uniformização, razão $U_{\min } / U_{\max }$.

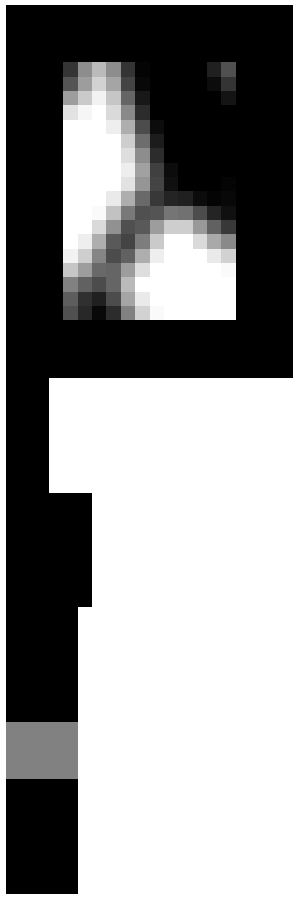

(a) $w_{2}=20$

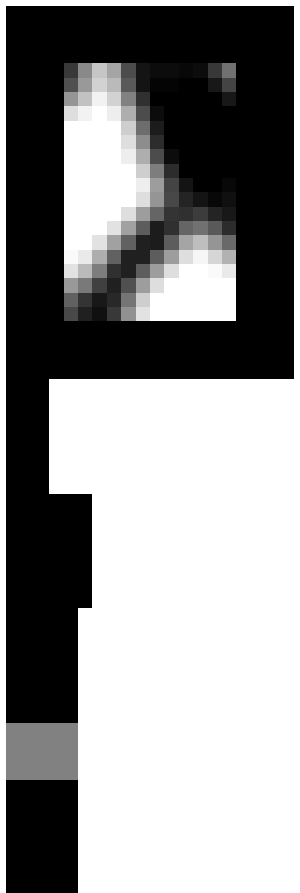

(b) $w_{2}=50$

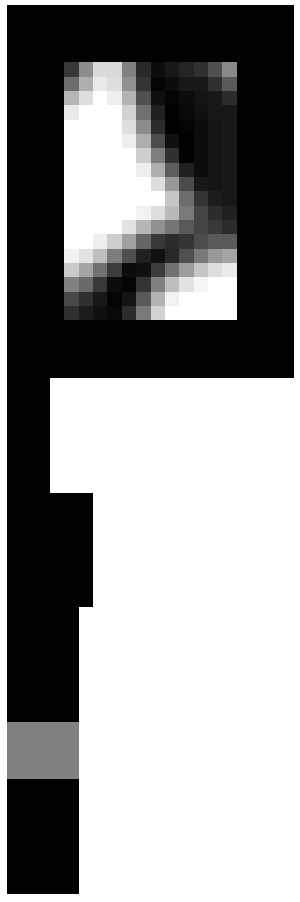

(c) $w_{2}=70$

Figura 6.18: Topologias ótimas para $w_{1}=300, w_{3}=450, w_{4}=280, d_{0}=0,45$, $\Theta_{\text {lim_sup }}=47 \%, \beta=0,027 \mu \mathrm{m}$ e $R_{\max }=5,5 \mathrm{~mm}$.

Os gráficos de convergência da função $L_{2}$ são apresentadas na Fig. 6.19 e mostram que ela foi maximizada. A razão entre as amplitudes mínima e máxima, $U_{\min } / U_{\max }$, que fornece o grau de uniformização das amplitudes, é apresentada na Fig. 6.20 e a Fig. 6.21 mostra a configuração dos deslocamentos na face superior do sonotrodo.

Ao final da otimização, os casos (a), (b) e (c) resultaram em uma uniformização de 60, 57\%, 72, 42\% e 17,61\%, respectivamente. Então, analisando os gráficos da Fig. 6.21 nota-se novamente um aumento na uniformização do caso $w_{2}=20$ para $w_{2}=50$, mas uma 


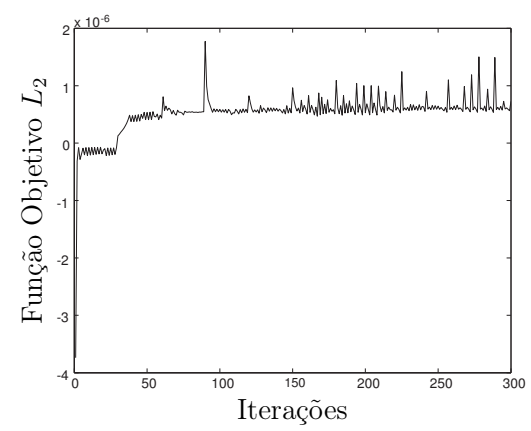

(a) $w_{2}=20$

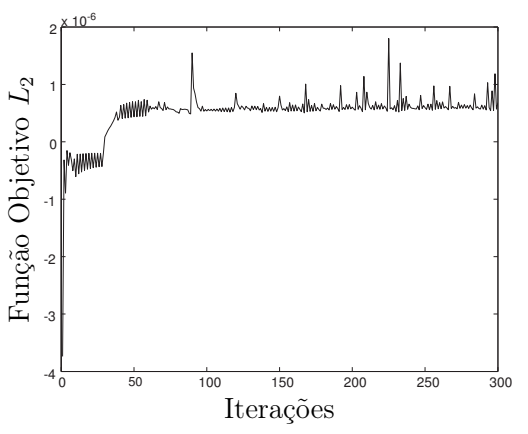

(b) $w_{2}=50$

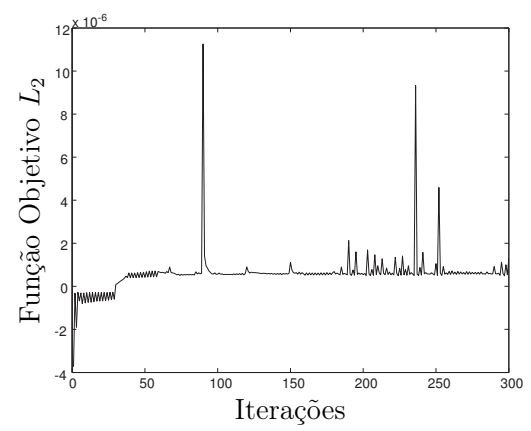

(c) $w_{2}=70$

Figura 6.19: Gráficos de convergência da função $L_{2}$.

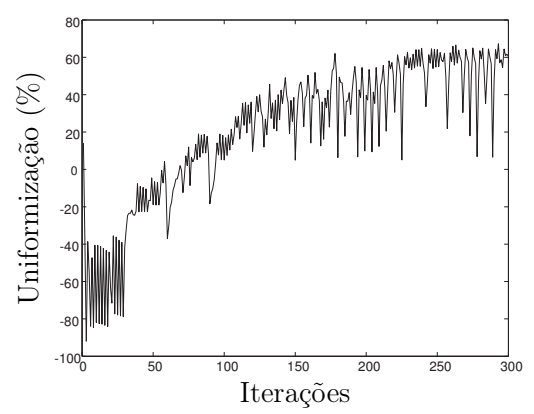

(a) $w_{2}=20$

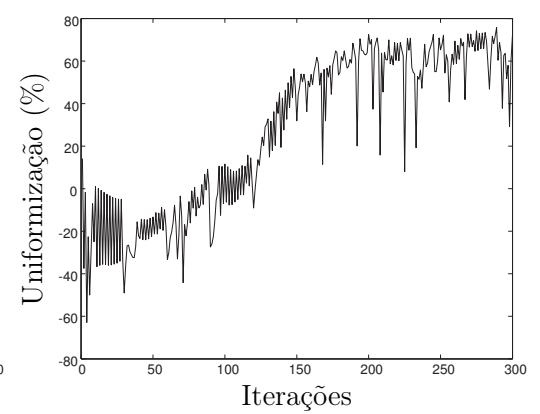

(b) $w_{2}=50$

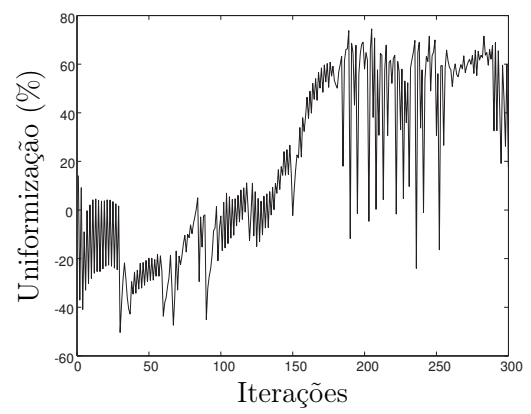

(c) $w_{2}=70$

Figura 6.20: Gráficos da uniformização, razão $U_{\min } / U_{\max }$.

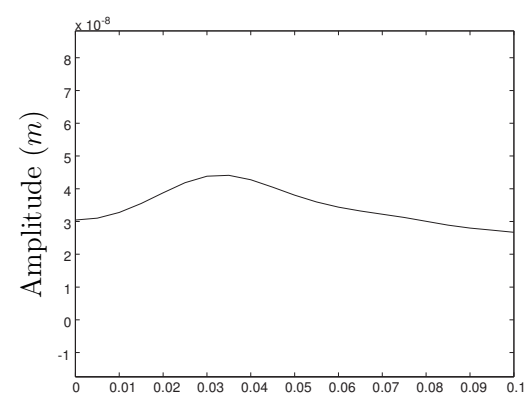

(a) $w_{2}=20$

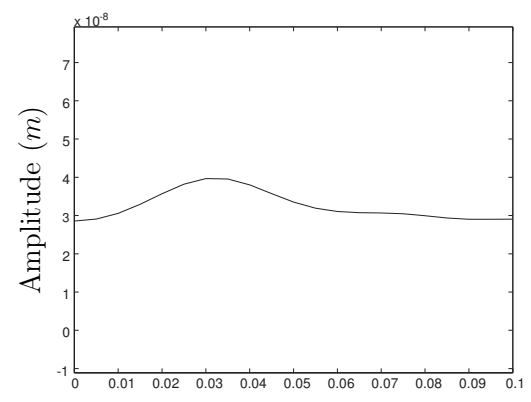

(b) $w_{2}=50$

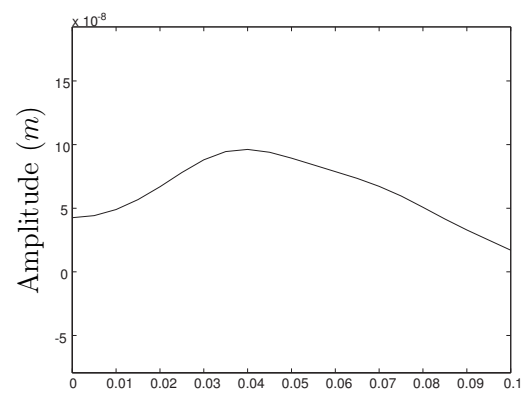

(c) $w_{2}=70$

Figura 6.21: Configuração dos deslocamentos na face de trabalho do sonotrodo. A abscissa representa o comprimento do sonotrodo em $m$.

diminuição da uniformização com $w_{2}=70$. Contudo, como $w_{2}$ prioriza a maximização, nota-se pela Fig. 6.21 que, em média, os deslocamentos são maximizados ao aumentar o peso $w_{2}$.

Analisando os gráficos da Fig. 6.20 observa-se que, quanto maior $w_{2}$, mais instavél se 
apresenta a razão $U_{\min } / U_{\max }$. Isso ocorre pelo fato de que a função $L_{2}$ também é dinâmica, possuindo uma sensibilidade alta em relação às outras funções. Como exemplo, para o caso $w_{2}=70$, apesar de atingir uniformização com valores mais altos do que os outros casos durante a otimização, ao final do processo, a razão $U_{\min } / U_{\max }$ foi igual a $17,61 \%$. Portanto, agora conclui-se que existe uma relação de interdependência, não somente entre as funções $L_{1}$ e $L_{3}$, mas também com relação à $L_{2}$.

\subsubsection{Influência do coeficiente $w_{3}$}

O coeficiente de peso $w_{3}$ permite variar rigidez da estrutura. Portanto, variando $w_{3}$ obtem-se diferentes resultados do MOT, como mostrados na Fig. 6.22, considerando os valores de $w_{3}$ iguais a 300, 400 e 500, respectivamente. Os demais pesos $w_{1}, w_{2}$ e $w_{4}$ são iguais à 300, 50, 280 respectivamente, e os valores de $d_{0}, \Theta_{\text {lim_sup }}, \beta$ e $R_{\text {max }}$ são iguais a 0, 45, 47\%, 0, $027 \mu \mathrm{m}$ e 5, $5 \mathrm{~mm}$, respectivamente.

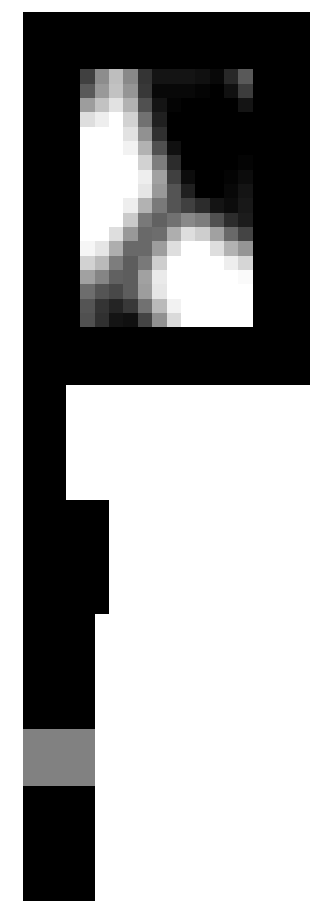

(a) $w_{3}=300$

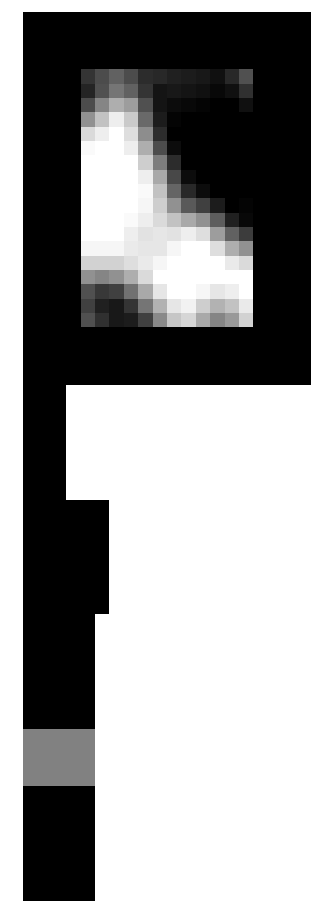

(b) $w_{3}=400$

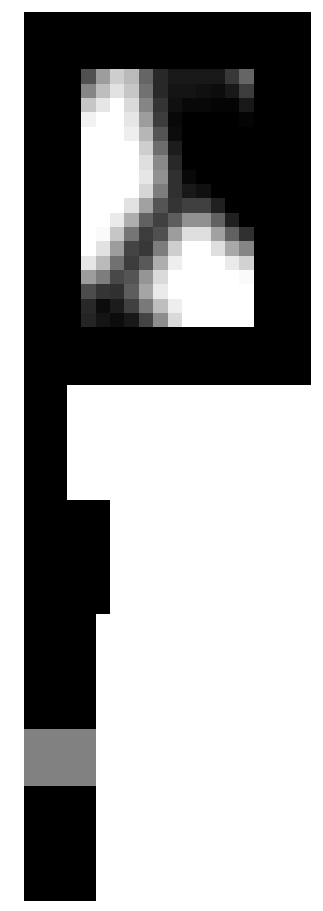

(c) $w_{3}=500$

Figura 6.22: Topologias ótimas para $w_{1}=300, w_{2}=50, w_{4}=280, d_{0}=0,45$, $\Theta_{\text {lim_sup }}=47 \%, \beta=0,027 \mu \mathrm{m}$ e $R_{\max }=5,5 \mathrm{~mm}$.

A Fig. 6.23 mostra as deformadas apenas dos sonotrodos das topologias ótimas obtidas na Fig. 6.22, e a configuração dos deslocamentos na face superior do sonotrodo é apresentada na Fig. 6.24 . 


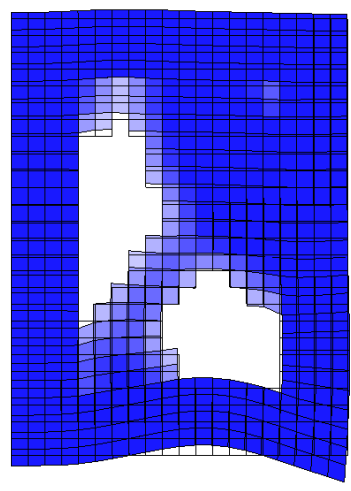

(a) $w_{3}=300$

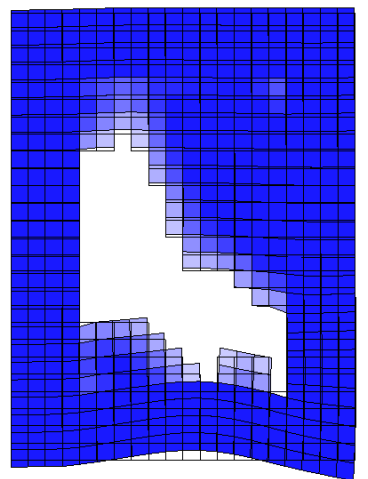

(b) $w_{3}=400$

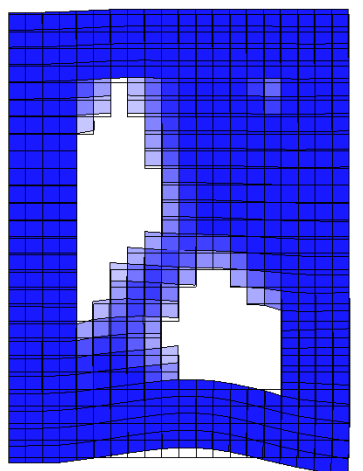

(c) $w_{3}=500$

Figura 6.23: Configuração deformada apenas dos sonotrodos das topologias ótimas mostradas na Fig. 6.22, excitadas a $20 \mathrm{kHz}$.

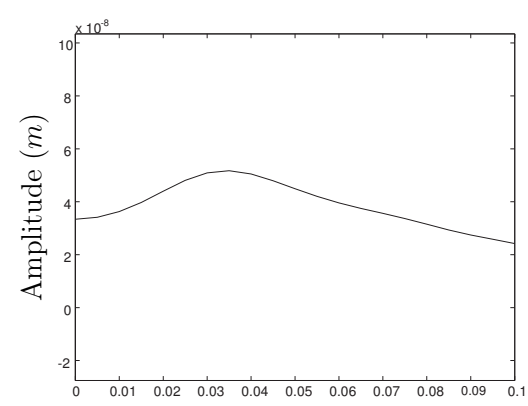

(a) $w_{3}=300$

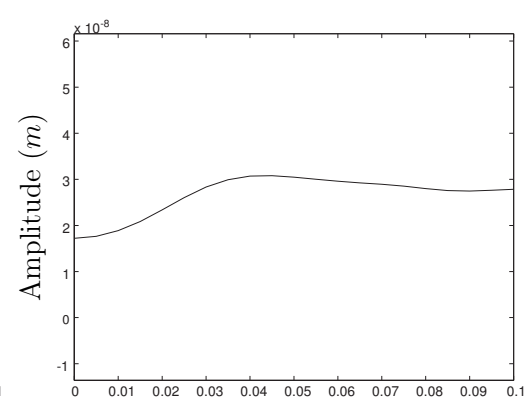

(b) $w_{3}=400$

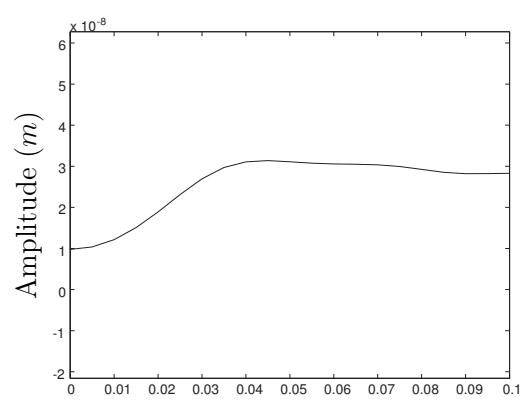

(c) $w_{3}=500$

Figura 6.24: Configuração dos deslocamentos na face de trabalho do sonotrodo. A abscissa representa o comprimento do sonotrodo em $m$.

Os gráficos de convergência da função $L_{3}$ são apresentadas na Fig. 6.25 e, embora ela tenha sido maximizada, observa-se que nos intervalos de picos causados pela mudança nas penalizações do modelo de material, a função é minimizada.

Ao final da otimização, os casos (a), (b) e (c) resultaram em uma uniformização de $46,79 \%, 55,84 \%$ e 31, 22\%, respectivamente. Observa-se pelas deformadas da estrutura apresentadas na Fig. 6.23 que ao aumentar o peso $w_{3}$ a estrutura fica mais rígida, resultando em deslocamentos de menor amplitue, comprovando a funcionalidade da formulação. Porém, novamente temos uma relação de dependência entre os pesos, fazendo com que a uniformização seja maior para $w_{3}=400$, e para o caso em que $w_{3}=500$, a estrutura ficou rígida demais, diminuindo o valor da uniformização. 


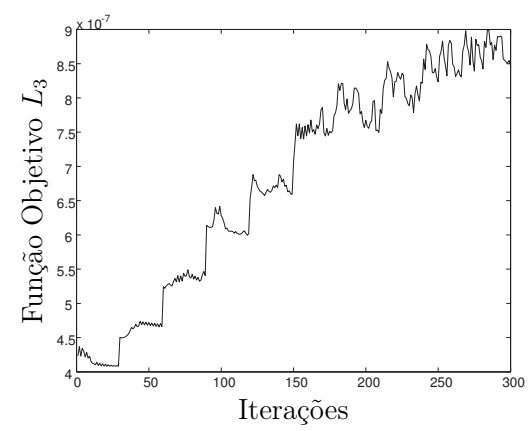

(a) $w_{3}=300$

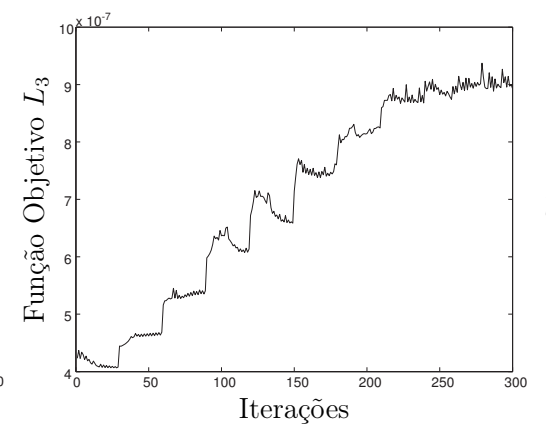

(b) $w_{3}=400$

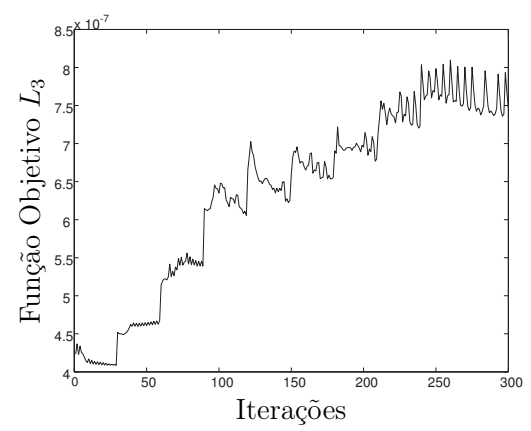

(c) $w_{3}=500$

Figura 6.25: Gráficos de convergência da função $L_{3}$.

\subsubsection{Influência do coeficiente $\boldsymbol{w}_{4}$}

O coeficiente de peso $w_{4}$ permite variar a prioridade da função multi-objetivo em relação à função da freqüência de ressonância. A freqüência alvo $\left(f_{0}\right.$ da Eq. (4.7)) é igual a $24 k H z$, pois a freqüência do modo de pistão deve estar acima de $20 k H z$. Para essa função, é também necessário determinar o modo desejado a ser procurado, que para este caso, é o modo de pistão, apresentado na Fig. 6.8 .

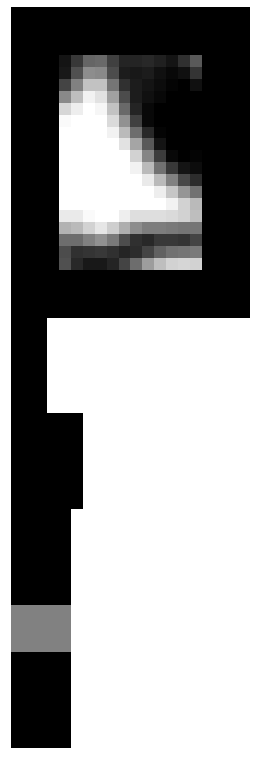

(a) $w_{4}=200$

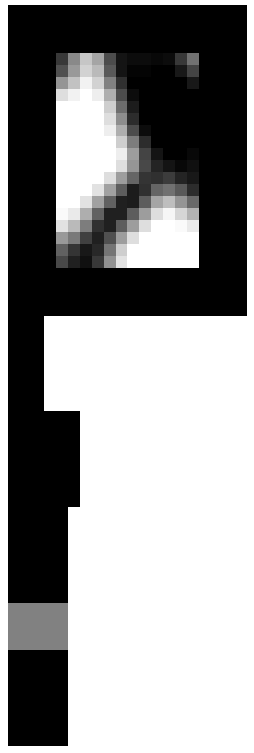

(b) $w_{4}=280$

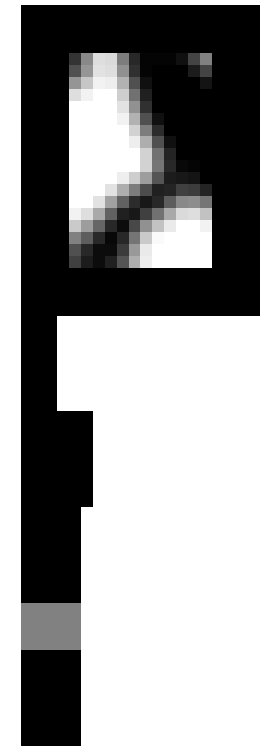

(c) $w_{4}=350$

Figura 6.26: Topologias ótimas para $w_{1}=300, w_{2}=50, w_{3}=450, d_{0}=0,45$, $\Theta_{\text {lim_sup }}=47 \%, \beta=0,027 \mu \mathrm{m}$ e $R_{\max }=5,5 \mathrm{~mm}$.

Então, variando $w_{4}$ obtem-se diferentes resultados do MOT, como mostrado na Fig. 6.26, considerando os valores de $w_{4}$ iguais a 200, 280 e 350, respectivamente. Os 
demais pesos $w_{1}, w_{2}$ e $w_{3}$ são iguais à $300,50,450$ respectivamente, e os valores de $d_{0}$, $\Theta_{\text {lim_sup }}, \beta$ e $R_{\max }$ são iguais a 0,45,47\%, 0,027 $\mu \mathrm{m}$ e 5,5 mm, respectivamente.

O modo alvo, obtido na primeira iteração é apresentado na Fig. 6.27(a) e as Figs. 6.27(b), (c) e (d) mostram os modos de pistão das topologias ótimas obtidas na Fig. 6.26, correspondendo às freqüências iguais a 13632, 20856 e $20955 \mathrm{~Hz}$, respectivamente. A diferença entre modos das Figs. 6.27(b), (c) e (d) e do modo alvo (Fig. 6.27(a)) é devido à diferença de fase do modo de vibrar. O que realmente importa é que os deslocamentos de todos os pontos da face superior do sonotrodo estejam vibrando em fase, ou seja, ou todos positivos, ou todos negativos.

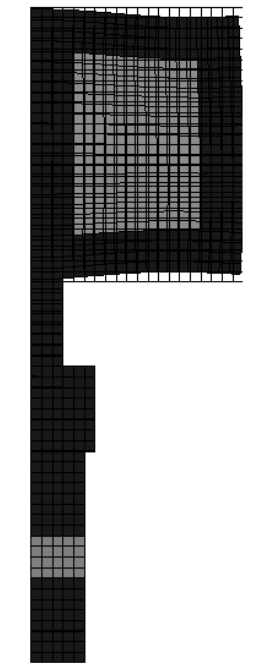

(a) Modo alvo

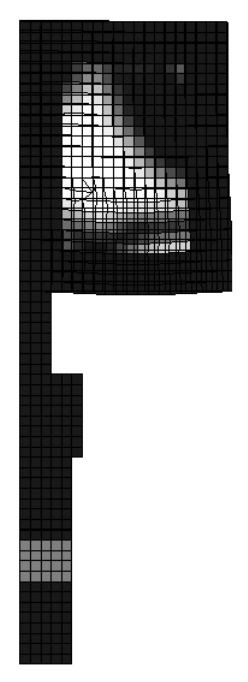

(b) $w_{4}=200$

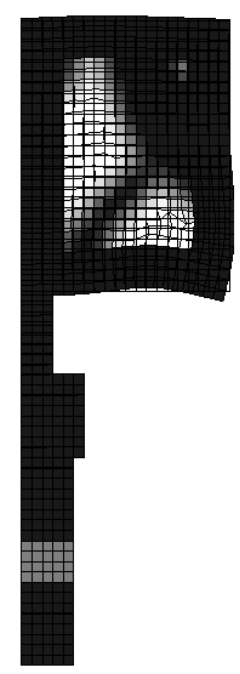

(c) $w_{4}=280$

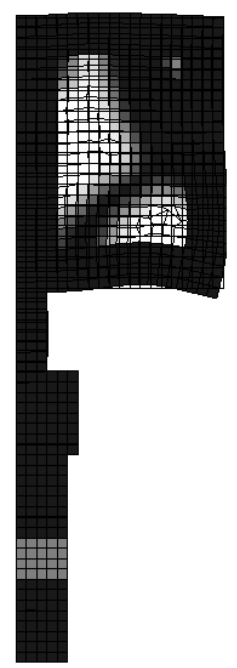

(d) $w_{4}=350$

Figura 6.27: Modo alvo e modo de pistão das topologias ótimas obtidas na Fig. 6.26.

Os gráficos de convergência da função $L_{4}$ são apresentadas na Fig. 6.28, mostrando que a escolha do peso $w_{4}$ influencia tanto na convergência da função quanto na convergência da freqüência do modo de pistão, apresentada pelos gráficos da Fig. 6.29.

Ao final da otimização, os casos (b), (c) e (d) da Fig. 6.27 apresentaram a freqüência do modo de pistão iguais a $13632 \mathrm{~Hz}, 20856 \mathrm{~Hz}$ e $20955 \mathrm{~Hz}$, respectivamente, e a uniformização resultante foi igual a 53,58\%, 72,04\% e 48,05\%, respectivamente. Ou seja, o valor de $w_{4}$, além de influenciar na convergência da freqüência do modo de pistão, também influencia na uniformização dos deslocamentos. Observa-se que, ao flexibilizar a função multi-objetivo em relação à $L_{4}$, diminuindo o valor de $w_{4}$ para 200 , a uniformização 


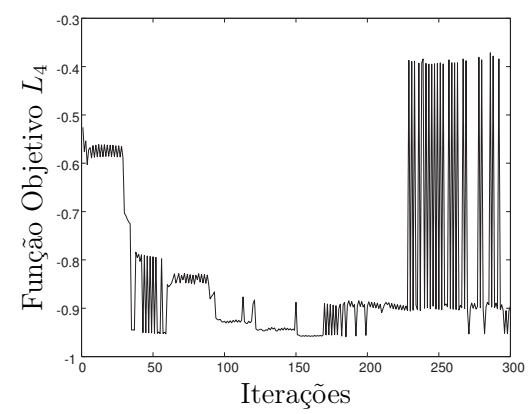

(a) $w_{4}=200$

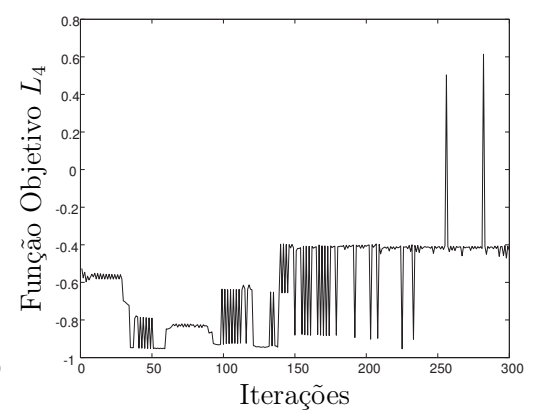

(b) $w_{4}=280$

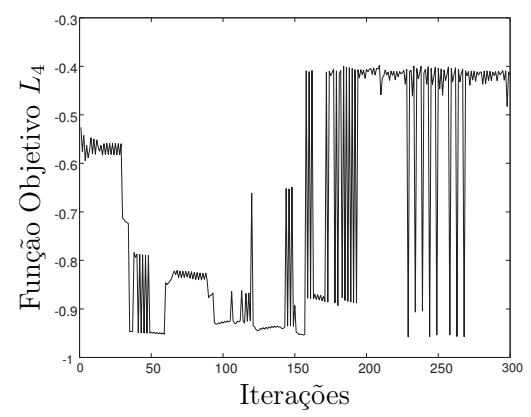

(c) $w_{4}=350$

Figura 6.28: Gráficos de convergência da função $L_{4}$.

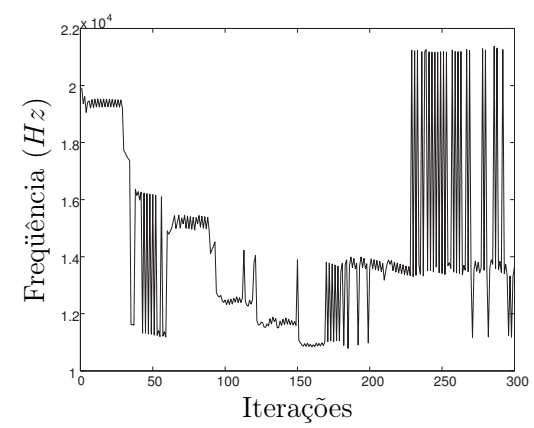

(a) $w_{4}=200$

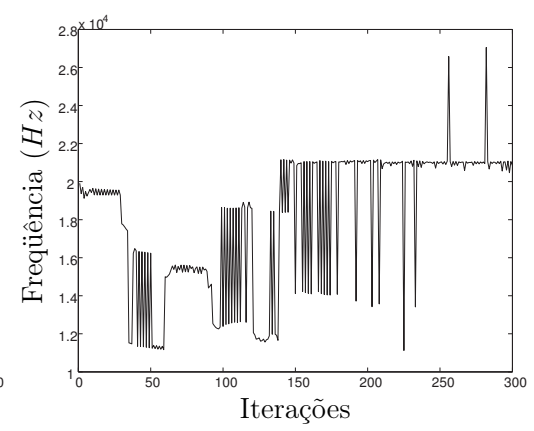

(b) $w_{4}=280$

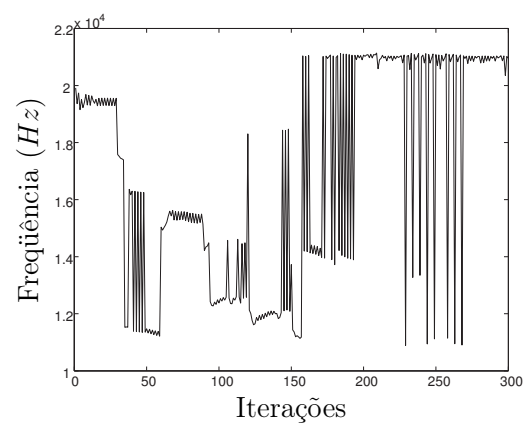

(c) $w_{4}=350$

Figura 6.29: Gráficos de convergência da freqüência do modo de pistão.

é maior do que no caso $w_{4}=350$, porém, a freqüência passa a oscilar mais, resultando em valores mais baixos para a freqüência do modo de pistão.

\subsubsection{Influência do Valor Inicial das Variáveis de Projeto $\left(d_{0}\right)$}

O objetivo deste estudo é mostrar a sensibilidade do MOT em relação ao valor inicial das variáveis de projeto, uma vez que este problema possui vários mínimos locais, e portanto, o resultado depende dos valores iniciais atribuídos às variáveis de projeto $d_{0}$. Assim, a Fig. 6.30 ilustra as diferentes topologias ótimas obtidas utilizando valores de $d_{0}$ iguais a $0,30,0,38$ e 0,45, e os demais parâmetros iguais a $w_{1}=300, w_{2}=50, w_{3}=450$, $w_{4}=280, \beta=0,027 \mu \mathrm{m}, \Theta_{\text {lim_sup }}=47 \%$, e $R_{\max }=5,5 \mathrm{~mm}$.

Os gráficos da Fig. 6.31 apresentam a influência de $d_{0}$ na uniformização dos deslocamentos ao final da otimização, resultando em 68,95\%, 40,87\% e 72,04\% para os casos (a), (b) e (c), respectivamente.

Observa-se pela Fig. 6.30 que a topologia dos resultados do MOT possui sensibilidade 


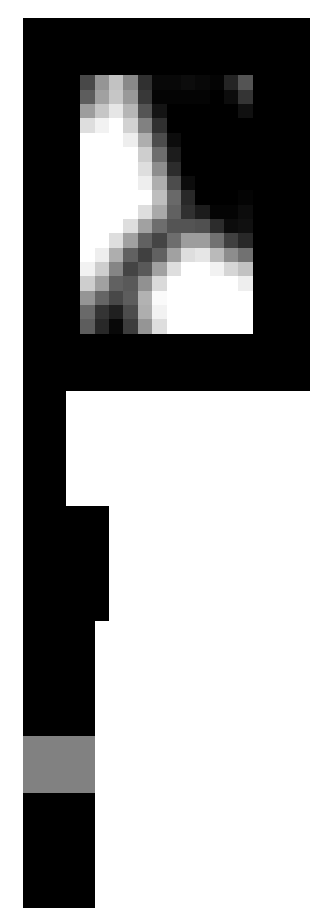

(a) $d_{0}=0,30$

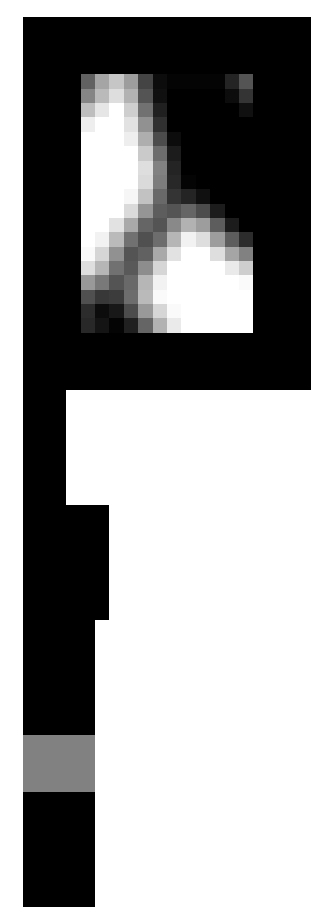

(b) $d_{0}=0,38$

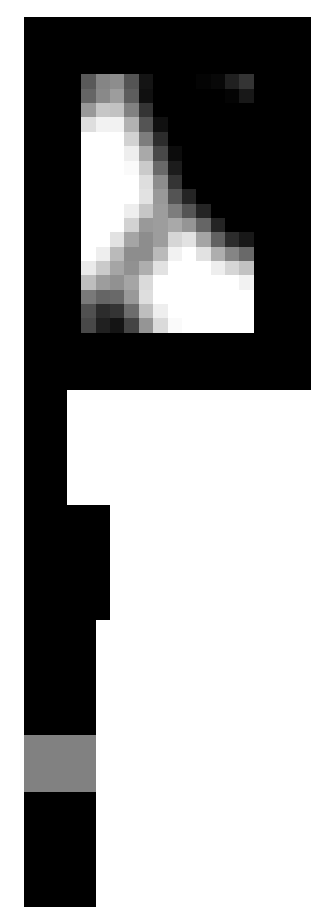

(c) $d_{0}=0,45$

Figura 6.30: Topologias ótimas utilizando diferentes valores para $d_{0}$.

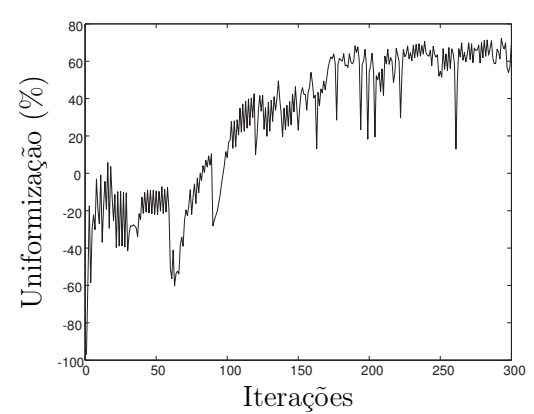

(a) $d_{0}=0,30$

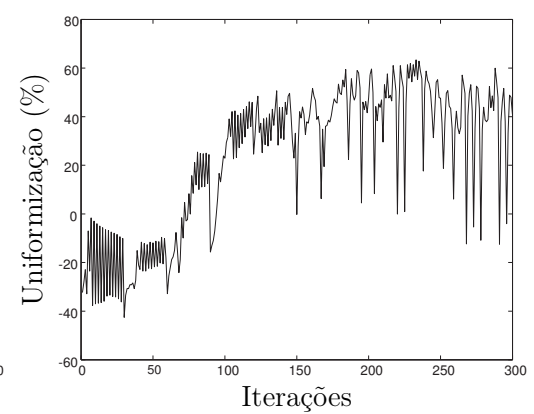

(b) $d_{0}=0,38$

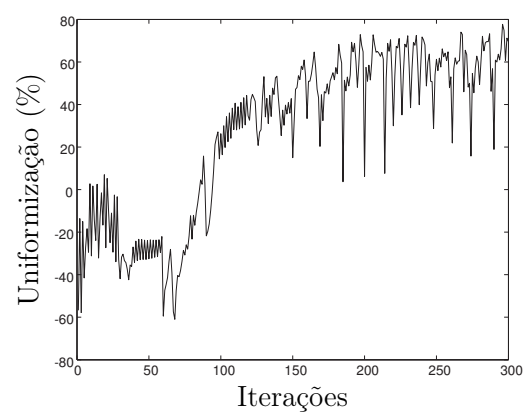

(c) $d_{0}=0,45$

Figura 6.31: Gráficos da uniformização, razão $U_{\min } / U_{\max }$.

baixa em relação ao valor inicial da variável de projeto, pois as topologias não diferem muito entre si. Contudo, analisando a Fig. 6.31, percebe-se que a uniformização é mais sensível, resultando em valores muito diferentes entre as topologias obtidas.

\subsubsection{Influência do Raio do Filtro $\left(R_{\max }\right)$}

O objetivo deste estudo é mostrar a sensibilidade do MOT em relação ao raio do filtro de projeção utilizado. Assim, a Fig. 6.32 ilustra as diferentes topologias ótimas obtidas 
utilizando valores de $R_{\max }$ iguais a 5,5, 11 e $16 \mathrm{~mm}$, e os demais parâmetros iguais a $w_{1}=300, w_{2}=50, w_{3}=450, w_{4}=280, \beta=0,027 \mu m, d_{0}=0,45$ e $\Theta_{\text {lim_sup }}=47 \%$.

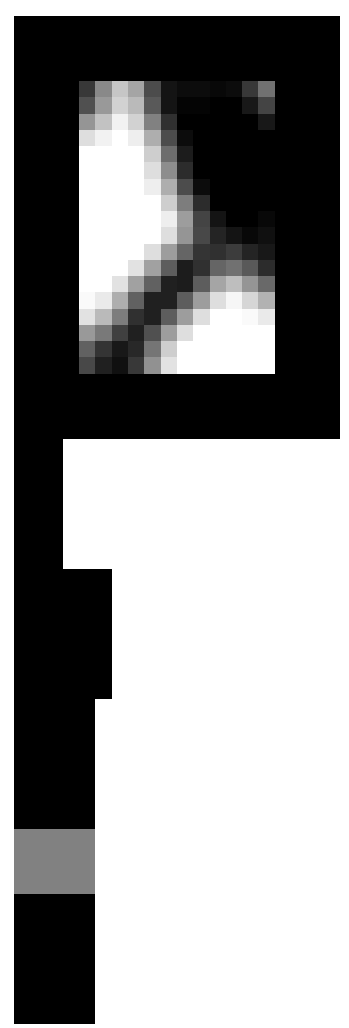

(a) $R_{\max }=5,5 \mathrm{~mm}$

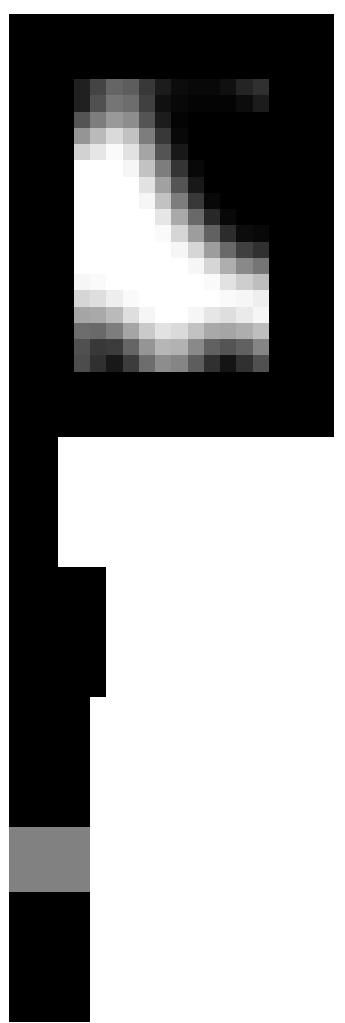

(b) $R_{\max }=11 \mathrm{~mm}$

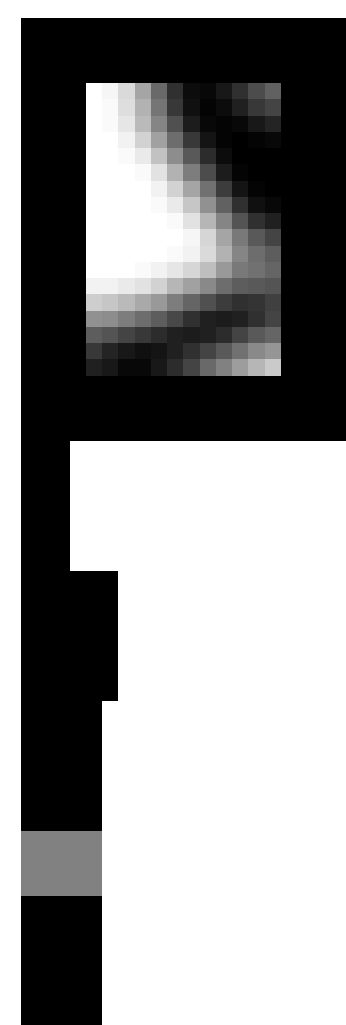

(c) $R_{\max }=16 \mathrm{~mm}$

Figura 6.32: Topologias ótimas utilizando diferentes valores para $R_{\max }$.

Os gráficos da Fig. 6.33 apresentam a influência de $R_{\max }$ na uniformização dos deslocamentos ao final da otimização, resultando em $72,04 \%, 41,98 \%$ e $8,13 \%$ para os casos (a), (b) e (c), respectivamente.

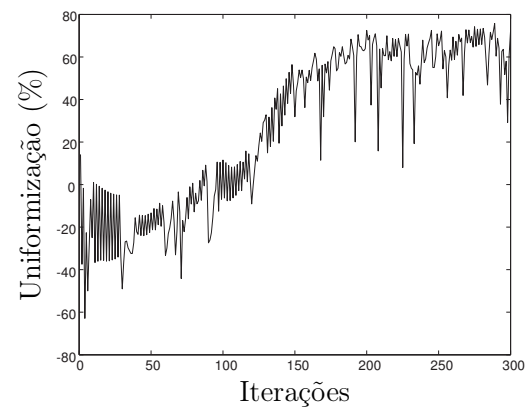

(a) $R_{\max }=5,5 \mathrm{~mm}$

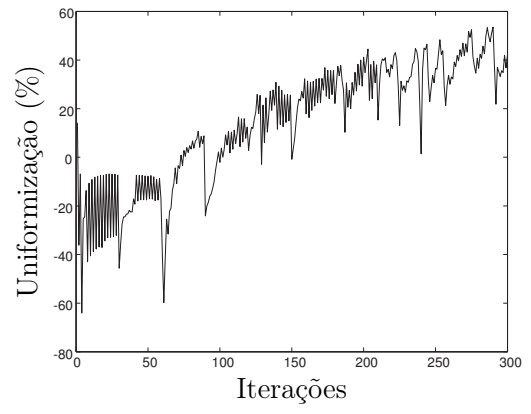

(b) $R_{\max }=11 \mathrm{~mm}$

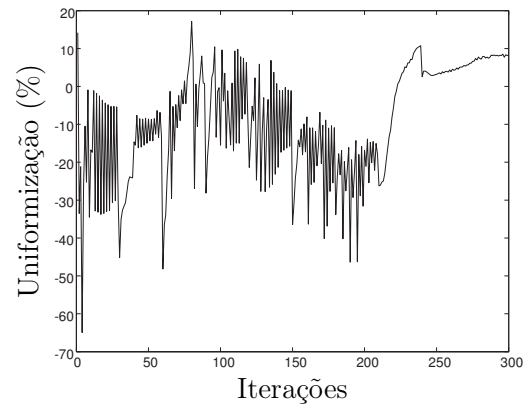

(c) $R_{\max }=16 \mathrm{~mm}$

Figura 6.33: Gráficos da uniformização, razão $U_{\min } / U_{\max }$. 
resultante muda completamente, apresentando maior número de elementos intermediários ("escala de cinza") e alterando o resultado da uniformização.

\subsubsection{Influência da Restrição de Volume Superior $\left(\Theta_{\text {lim_sup }}\right)$}

O objetivo deste estudo é mostrar a sensibilidade do MOT em relação à restrição de volume superior utilizada. Assim, a Fig. 6.34 ilustra as diferentes topologias ótimas obtidas utilizando valores de $\Theta_{\text {lim_sup }}$ iguais a $0,40,0,47$ e 0,55, e os demais parâmetros iguais a $w_{1}=300, w_{2}=50, w_{3}=450, w_{4}=280, \beta=0,027 \mu m, d_{0}=0,45$ e $R_{\max }=$ $5,5 \mathrm{~mm}$.

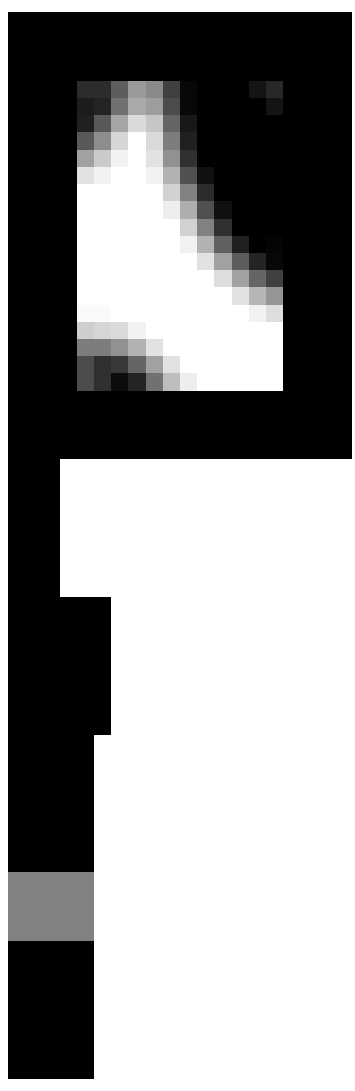

(a) $\Theta_{\text {lim_sup }}=0,40$

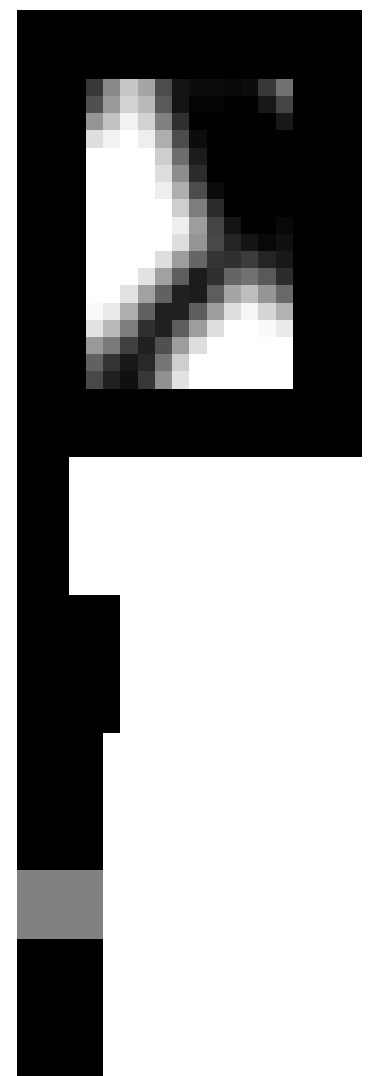

(b) $\Theta_{\text {lim_sup }}=0,47$

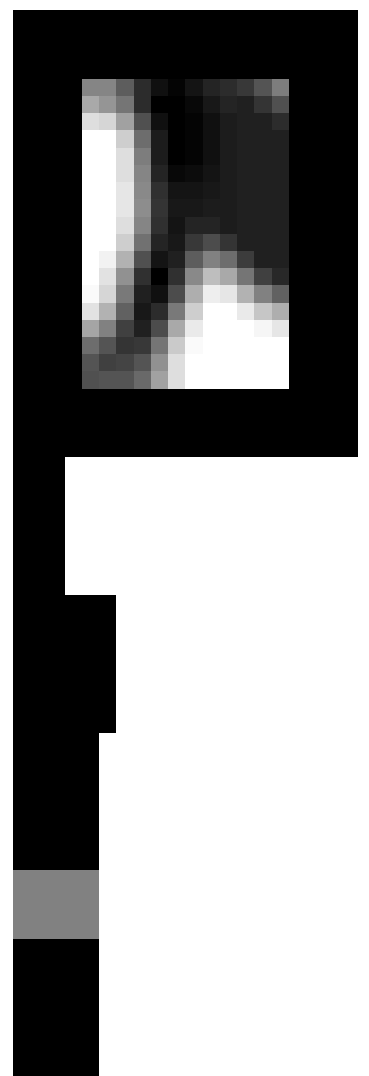

(c) $\Theta_{\text {lim_sup }}=0,55$

Figura 6.34: Topologias ótimas utilizando diferentes valores para $\Theta_{\text {lim_sup }}$.

Os gráficos da Fig. 6.35 apresentam a influência de $\Theta_{\text {lim_sup na uniformização dos }}$ deslocamentos ao final da otimização, resultando em 50,24\%, 72, $04 \%$ e -21, $2 \%$ para os casos (a), (b) e (c), respectivamente.

Neste exemplo, observa-se que o resultado também muda ao se utilizar diferentes valores para $\Theta_{\text {lim_sup }}$, apresentando maior estabilidade na convergência da uniformização 


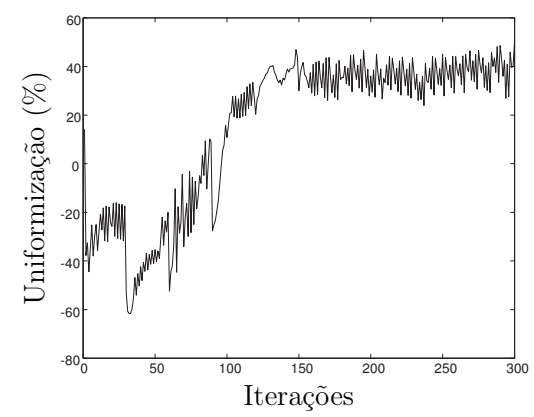

(a) $\Theta_{\text {lim_sup }}=0,40$

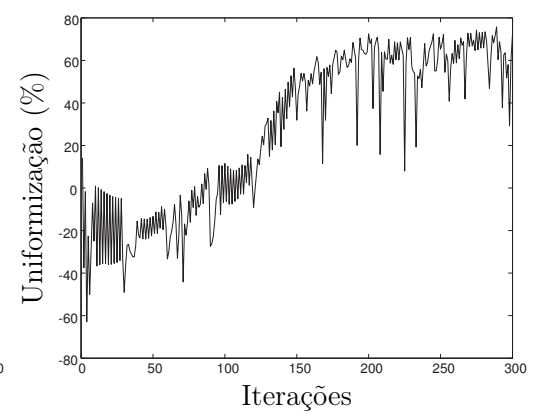

(b) $\Theta_{\text {lim_sup }}=0,47$

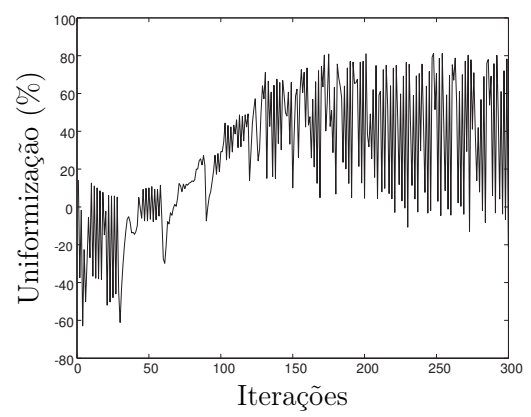

(c) $\Theta_{\text {lim_sup }}=0,55$

Figura 6.35: Gráficos da uniformização, razão $U_{\min } / U_{\max }$.

para valores mais baixos de $\Theta_{\text {lim_sup }}$, porém, valores maiores para $\Theta_{\text {lim_sup }}$ permitem atingir valores maiores para uniformização. Para o caso (c), por exemplo, utilizando $\Theta_{\text {lim_sup }}=0,55$, o valor final da uniformização foi o pior resultado, $-21,2 \%$, mas foi o o caso que apresentou uniformização maior que $80 \%$ durante a otimização.

\subsubsection{Conclusão sobre as Influências dos Parâmetros}

O estudo sobre a influência da variação dos parâmetros de otimização é importante para entendermos como cada parâmetro modifica o resultado individualmente. Observou-se que os parâmetros $w_{1}, \beta, R_{\max }$ e $\Theta_{\text {lim_sup }}$ têm mais influência sobre a uniformização do que os demais parâmetros, mas todos os parâmetros juntos têm papel importante na obtenção da topologia ótima.

Portanto, como o resultado depende de todos os parâmetros juntos, é difícil estabelecer os valores de todos os parâmetros para se obter o resultado ótimo, podendo existir soluções com melhores resultados daqueles apresentados neste trabalho.

\subsection{Resultados Numéricos Utilizando Domínio mais Discretizado}

As análises feitas na seção anterior trouxeram profundo entendimento da sensibilidade da função multi-objetivo em relação a todos os parâmetros de otimização, permitindo a obtenção dos resultados cada vez melhores. Os exemplos apresentados nesta seção utilizam o mesmo domínio utilizado nos exemplos anteriores (Fig. 6.7(a)), partindo com os mesmos valores para as variáveis de projeto. 


\subsubsection{Exemplo 1}

O primeiro resultado apresentado nesta seção utiliza os mesmos parâmetros da etapa de verificação da formulação e do programa implementado (Seção 6.4.1), e sua topologia é apresentada na Fig. 6.36(a) juntamente com a topologia utilizando 770 elementos Fig. 6.36(b). Com o mesmo intuito de melhorar a convergência da formulação utilizada na seção de validação (Seção 6.4.1), os limites móveis são reduzidos se a uniformização $\left(U_{\min } / U_{\max }\right)$ atingir valores maiores que um certo valor. Então, inicia-se com os valores dos limites móveis iguais a 15\%. Acima de 200 iterações, se a uniformização atingir um valor maior que 70\%, os limites móveis são reduzidos para 10\%, e acima de 250 iterações, o valor dos limites móveis são reduzidos para $5 \%$ se a uniformização ultrapassar $80 \%$.

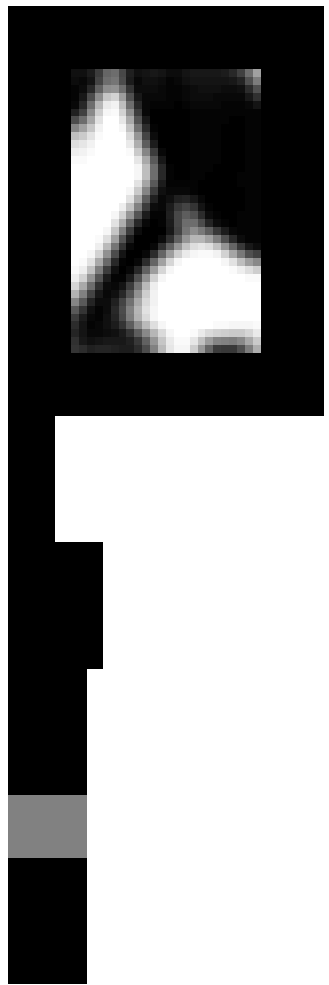

(a) 2768 elementos

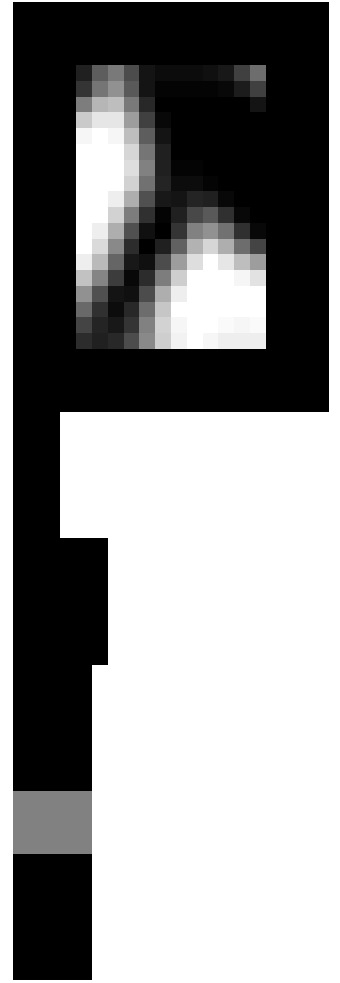

(b) 770 elementos

Figura 6.36: Topologias ótimas utilizando diferentes discretizações, com $w_{1}=300$, $w_{2}=40, w_{3}=450, w_{4}=280, d_{0}=0,45, \Theta_{\text {lim_sup }}=55 \%, \beta=0,027 \mu \mathrm{me}$ $R_{\max }=5,5 \mathrm{~mm}$.

Observa-se que as duas topologias não são muito diferentes uma da outra. A pequena diferença apresentada é devido ao nível de detalhamento que cada discretização permite visualisar, e portanto, conclui-se que o resultado não depende da malha ao se utilizar a técnica de projeção. 
No entanto, como já explorado nas análises feitas anteriormente, a uniformização e todas as demais funções são bastante sensíveis em relação à variação das variáveis de projeto. Por isso, ao final das 350 iterações, que também foram utilizadas para o exemplo da Fig. 6.36(b), a uniformização do resultado utilizando 2768 elementos, não ultrapassa o limite mínimo de uniformização, que é igual a 80\%. Mas se deixarmos a otimização seguir até 500 iterações, chega-se a um valor de uniformização igual à 84,96\%, como pode ser visto no gráfico de convergência da uniformização da Fig. 6.37(a). A freqüência do modo de pistão, ao final da otimização, apresentou seu valor igual à $21153 \mathrm{kHz}$, mostrada no gráfico de convergência da freqüência da Fig. 6.37(b).

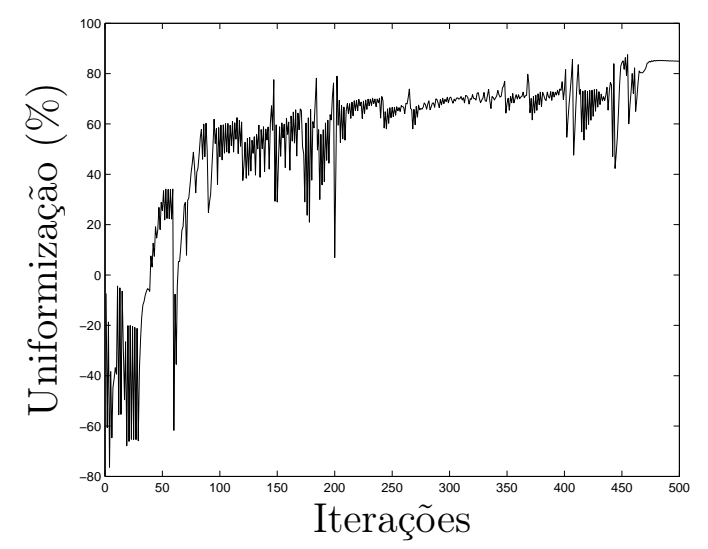

(a)

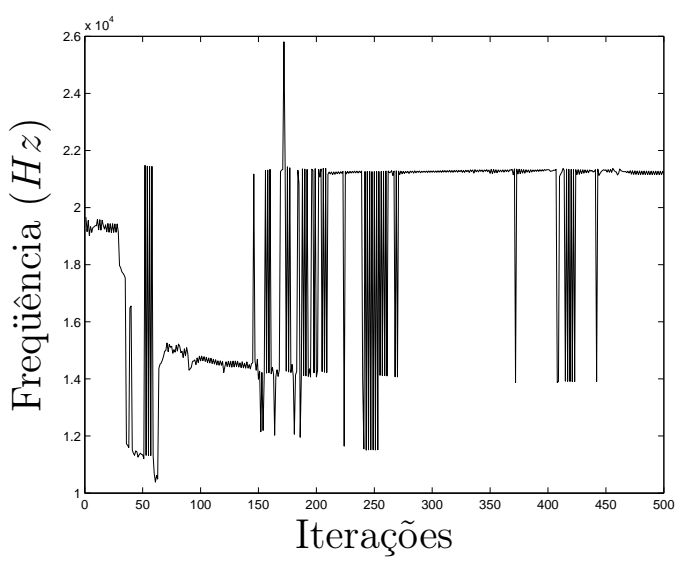

(b)

Figura 6.37: Gráficos de convergência da (a) uniformização, e (b) freqüência do modo de pistão do exemplo 1 .

Apesar da uniformização apresentar seu valor maior que $80 \%$ e a freqüência do modo de pistão estar acima de $20 \mathrm{kHz}$, ao ser feito o pós-processamento pelo programa implementado, os resultados de uniformização e freqüência podem ser alterados. O pós-processamento feito pelo programa implementado atribui o valor 1 para as variáveis de projeto cujos valores são maiores que uma certa tolerância estabelecida pelo projetista, e as demais variáveis tem seu valor reduzido à 0,001 (o valor zero não é utilizado para evitar instabilidades numéricas no cálculo do MEF). Como o resultado da uniformização é muito sensível em relação à variação das variáveis de projeto, o valor da uniformização obtido tende a ser diferente para a estrutura pós-processada.

Foi realizada uma varredura com diferentes valores de tolerância, desde 0,2 até 1, sendo que a estrutura que forneceu o valor máximo da uniformização, igual à $61,22 \%$, foi obtida utilizando tolerância de 0,57. Então, para esta tolerância, tem-se a topologia do sonotrodo pós-processada, sua deformada a $20 \mathrm{kHz}$, e o modo de pistão da estrutura pós-processada, 


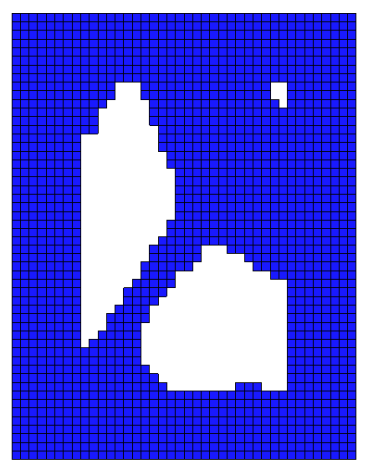

(a) Topologia

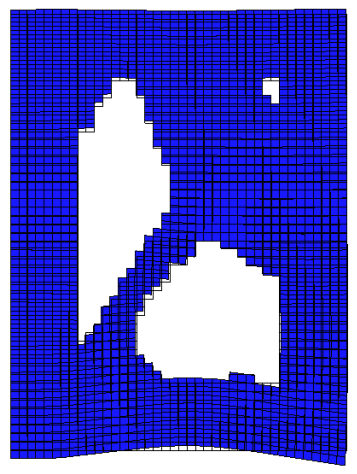

(b) Deformada

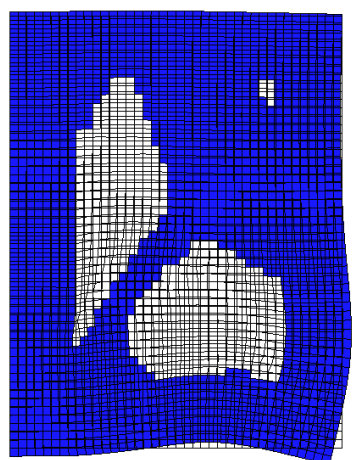

(c) Modo

Figura 6.38: (a) Topologia, (b) deformada e (c) modo de pistão do pós-processamento somente do sonotrodo da topologia apresentada no exemplo 1, omitindo o transdutor.

apresentadas na Fig. 6.38. A deformada e o modo foram obtidos utilizando o transdutor, mas como estamos interessados no sonotrodo, o transdutor é omitido nas figuras.

A partir do pós-processamento realizado pelo programa (Fig. 6.38(a)) é necessário um outro pós-processamento mais refinado, baseado no conhecimento e na intuição do projetista. Esse segundo pós-processamento é feito para aproximar as formas em degraus, provocada pelos elementos, em retas, como mostrado na Fig. 6.39(a).

As análises harmônica e modal desta nova estrutura são realizadas utilizando software comercial ANSYS ${ }^{\circledR}$. A deformada é apresentada na Fig. 6.39(b), a análise da configuração dos deslocamentos da face superior do sonotrodo, mostrada na Fig. 6.39(d), revela uma uniformização de 70,14\%, e a freqüência do modo de pistão, apresentado na Fig. 6.39(c), é igual a $21186 \mathrm{~Hz}$.

Portanto, como a uniformização não atingiu o valor mínimo de $80 \%$, conclui-se que esta topologia obtida não atende aos requisitos de projeto, não podendo ser utilizada para uma possível etapa de fabricação, por isso, a simulação em 3D não foi realizada. No entanto, a fim de aumentar a uniformização, as dimensões dos buracos da estrutura da Fig. 6.39(a) podem ser alteradas, ou seja, este resultado pode ser submetido à uma otimização paramétrica, refinando a topologia encontrada.

Então, para encontrar o resultado que atenda aos requisitos de projeto, um ou mais parâmetros da otimização podem ser alterados. Como este exemplo apresentou bons resultados, exceto pela etapa de pós-processamento, será realizada uma pequena a variação nos parâmetros, como mostrado pelo exemplo 2, na próxima Seção. 


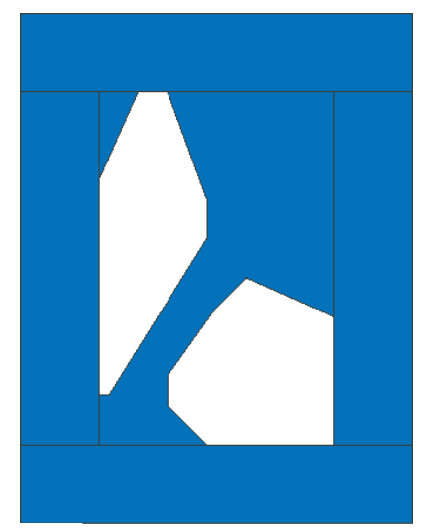

(a) Topologia

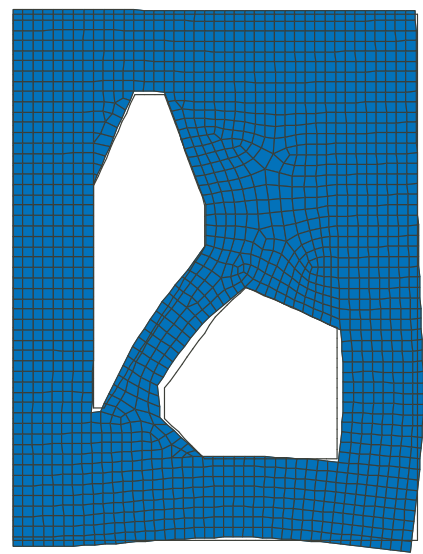

(b) Deformada

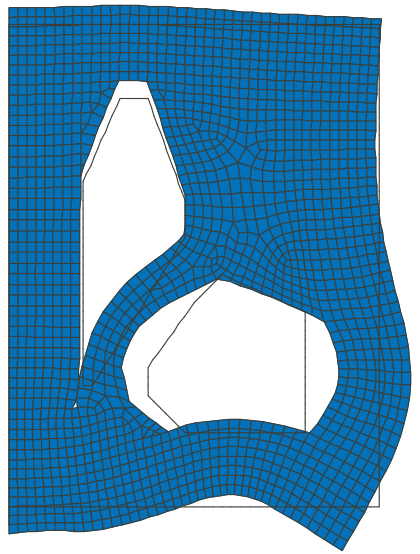

(c) Modo

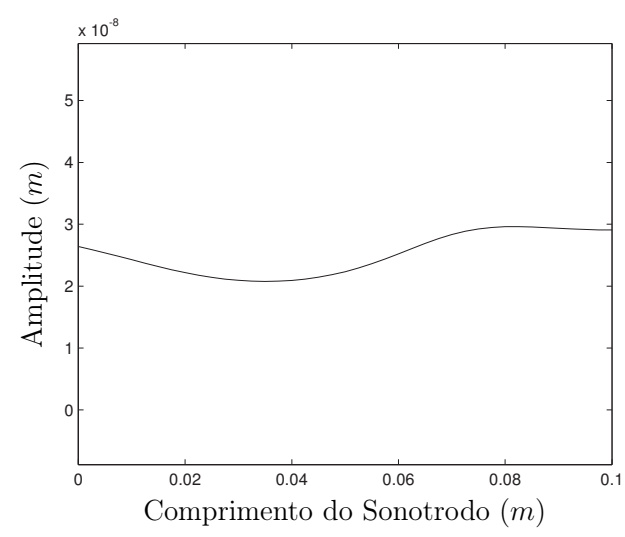

(d)

Figura 6.39: (a) Topologia, (b) deformada, (c) modo de pistão e (d) configuração dos deslocamentos do pós-processamento, em ANSYS ${ }^{\circledR}$, somente da topologia do sonotrodo do exemplo 1 , omitindo o transdutor.

\subsubsection{Exemplo 2}

Este segundo exemplo utiliza apenas uma modificação no parâmetro $w_{3}$, modificando-o para 430. O número de iterações permanece em 500. Com intuito de melhorar ainda mais a convergência da formulação utilizada, os limites móveis, iniciando em $15 \%$, são reduzidos para $1 \%$ se a uniformização $\left(U_{\min } / U_{\max }\right)$ atingir valores maiores que $70 \%$, após 200 iterações. A topologia do resultado ótimo obtido é apresentada na Fig. 6.40.

A uniformização resultante para este exemplo é igual a 80,46\%, como pode ser visto no gráfico de convergência da uniformização da Fig. 6.41(a). A freqüência do modo de pistão, ao final da otimização, é igual à $21215 \mathrm{kHz}$, apresentada no gráfico de convergência 


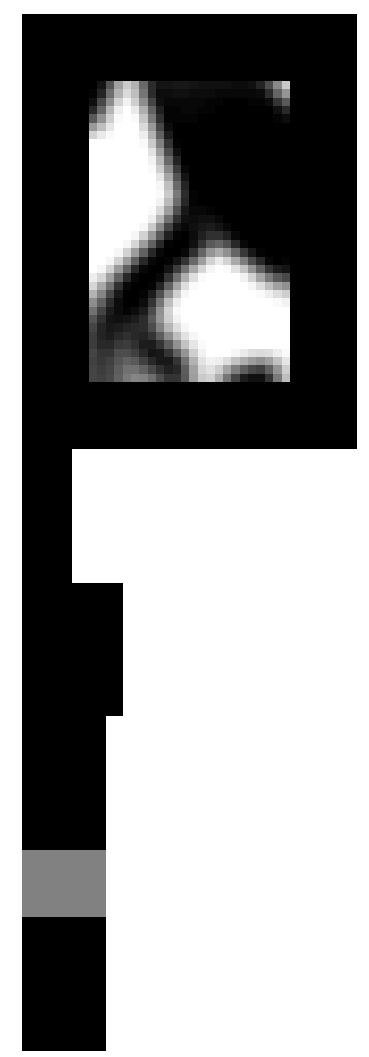

Figura 6.40: Topologia ótima do exemplo 2, com $w_{1}=300, w_{2}=40, w_{3}=430$, $w_{4}=280, d_{0}=0,45, \Theta_{\text {lim_sup }}=55 \%, \beta=0,027 \mu \mathrm{m}$ e $R_{\max }=5,5 \mathrm{~mm}$.

da freqüência da Fig. 6.41(b).

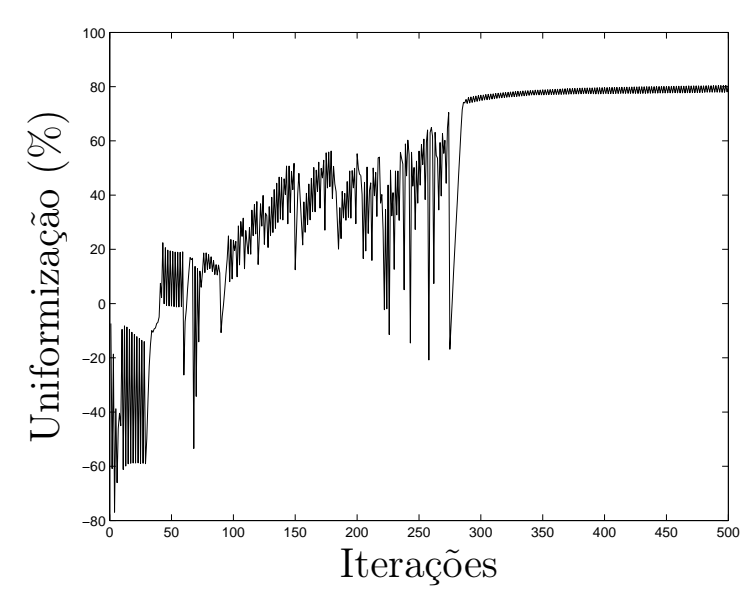

(a)

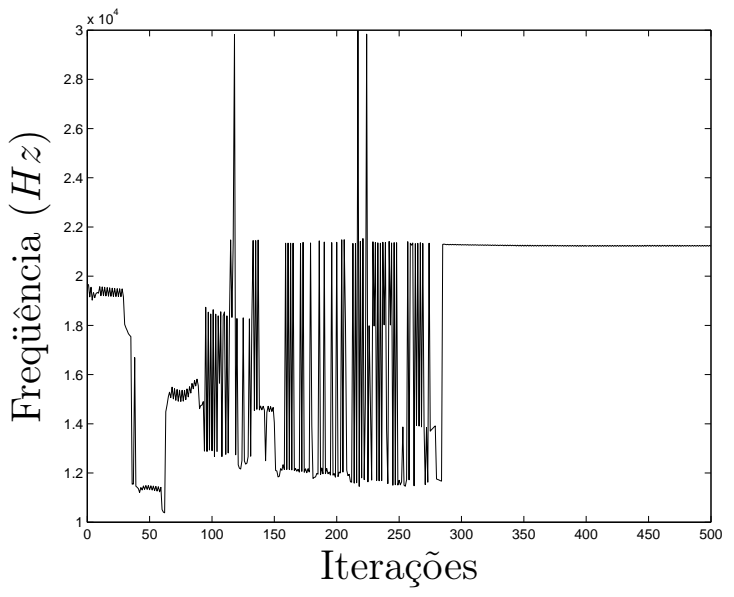

(b)

Figura 6.41: Gráficos de convergência da (a) uniformização, e (b) freqüência do modo de pistão do exemplo 2 .

A tolerância utilizada para o pós-processamento neste exemplo é igual a 0,62, o que resulta numa configuração de deslocamentos com uniformização igual a 81,50\%. O sonotrodo pós-processado, sua deformada a $20 \mathrm{kHz}$ e o modo de pistão são apresentados 
na Fig. 6.42, a seguir. A deformada e o modo foram obtidos utilizando o transdutor, mas como estamos interessados no sonotrodo, o transdutor é omitido nas figuras.

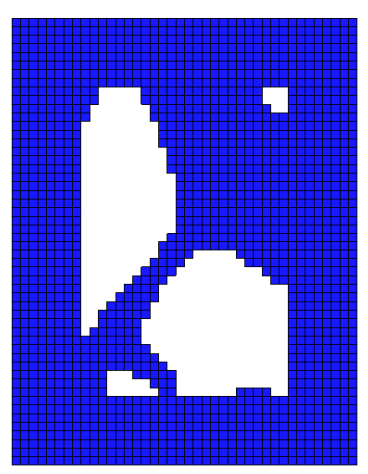

(a) Topologia

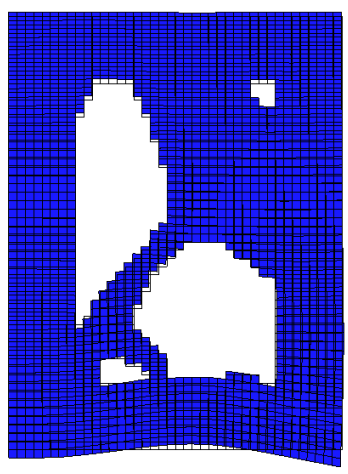

(b) Deformada

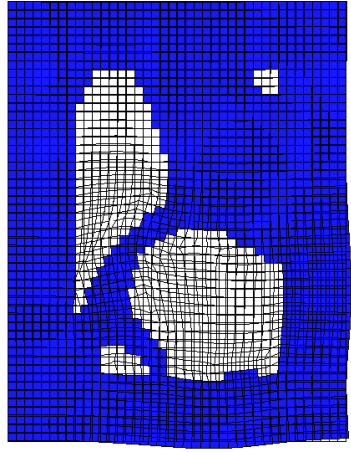

(c) Modo

Figura 6.42: (a) Topologia, (b) deformada e (c) modo de pistão do pós-processamento somente da topologia do sonotrodo do exemplo 2, omitindo o transdutor.

Do mesmo modo que no exemplo 1, o segundo pós-processamento, analisado pelo software comercial ANSYS ${ }^{\circledR}$, pode ser visto na Fig. 6.43(a). A deformada é apresentada na Fig. 6.43(b), a análise da configuração dos deslocamentos da face superior do sonotrodo, mostrada na Fig. 6.43(d), revela uma uniformização de $81,51 \%$, e a freqüência do modo de pistão, apresentado na Fig. 6.43(c), é igual a $21147 \mathrm{~Hz}$.

Assim, como os dois requisitos principais de projeto (uniformização e freqüência do modo de pistão) estão sendo obedecidos, é levantada a curva de impedância elétrica do conjunto para verificar se modo de pistão encontrado, cuja freqüência é igual a 21147 $H z$, é um modo piezelétrico (aquele que pode ser excitável), definido na seção 2.5 do Capítulo 2. De acordo com o gráfico da Fig. 6.44, no modo de pistão (21147 Hz), a impedância elétrica é mínima, o que caracteriza a freqüência de ressonância, o que era esperado pois foi utilizada a formulação MAC para seguir o modo desejado. O mesmo gráfico mostra que na freqüência de $20 \mathrm{kHz}$, a impedância elétrica tem o valor igual a $550 \Omega$, que é um valor baixo pois está próximo ao modo de pistão, sendo assim, uma freqüência excitável.

Portanto, foi encontrada a topologia ótima do sonotrodo que fornece mais de $80 \%$ de uniformização em sua face de trabalho, ao ser excitada em $20 \mathrm{kHz}$. A freqüência do seu modo de pistão também atendeu ao requisito de projeto, tendo seu valor acima de $20 k H z$. 


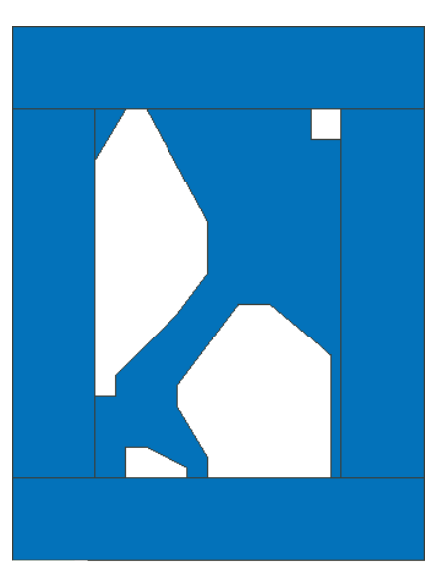

(a) Topologia

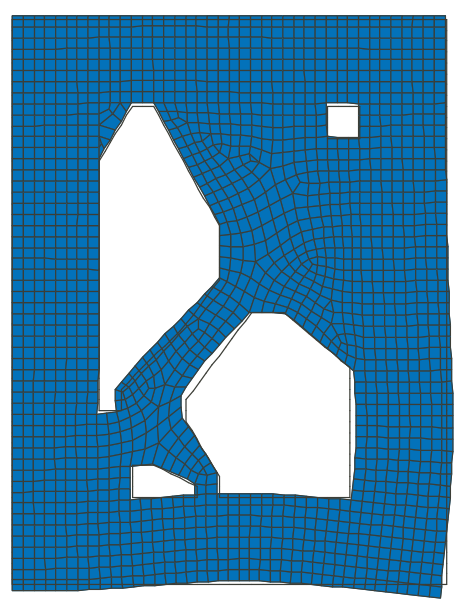

(b) Deformada

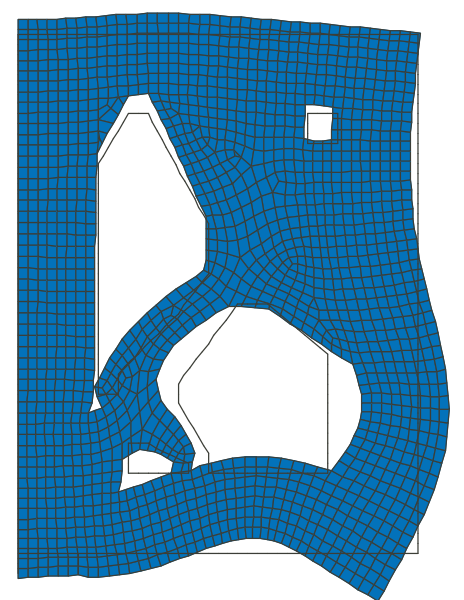

(c) Modo

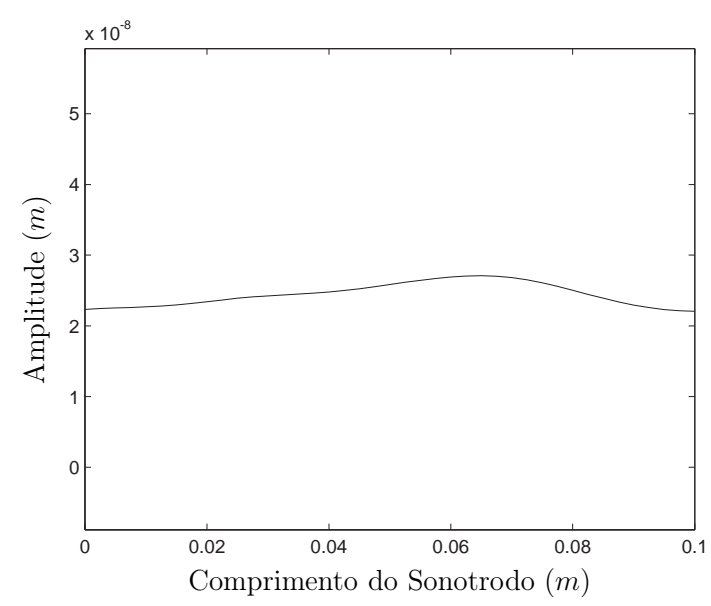

(d)

Figura 6.43: (a) Topologia, (b) deformada, (c) modo de pistão e (d) configuração dos deslocamentos do pós-processamento, obtido no ANSYS ${ }^{\circledR}$, somente da topologia do sonotrodo do exemplo 2, omitindo o transdutor.

Para finalizar as análises, é necessário verificar o resultado obtido. Então, foi construído um modelo 3D baseado na topologia 2D obtida. O modelo simétrico da estrutura completa, com o sonotrodo, amplificador e transdutor, pode ser visto na Fig. 6.45.

No entanto, a análise harmônica da estrutura em $20 \mathrm{kHz}$ revela uma uniformização de 73,51\%, ou seja, houve um decréscimo de $8 \%$ na uniformização da análise 2D para 3D. Houve também um decréscimo na freqüência de ressonância do modo de pistão, de $21147 \mathrm{~Hz}$ no modelo 2D para $20728 \mathrm{~Hz}$ no modelo 3D. Assim, apesar da freqüência do modo de pistão ainda estar acima do valor limite estipulado $(20 k H z)$, a uniformização, 


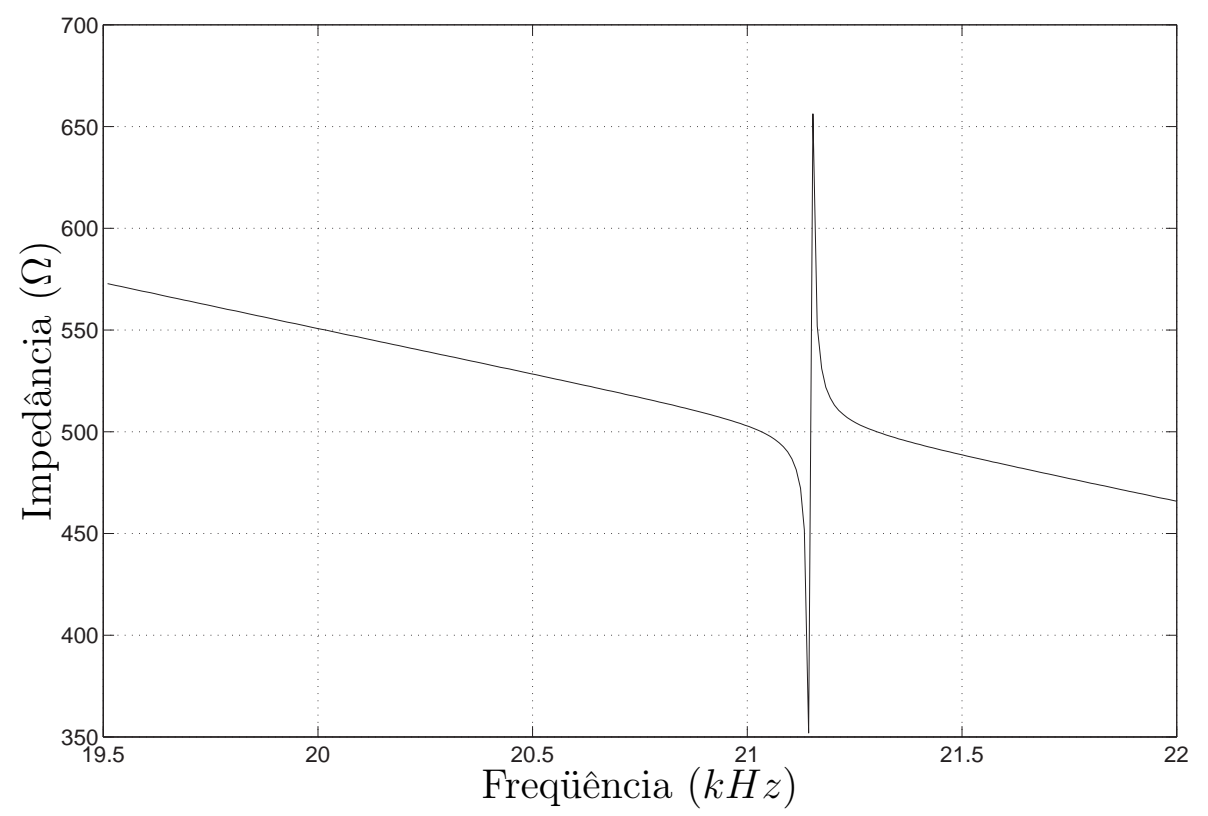

Figura 6.44: Gráfico de impedância do exemplo 2.

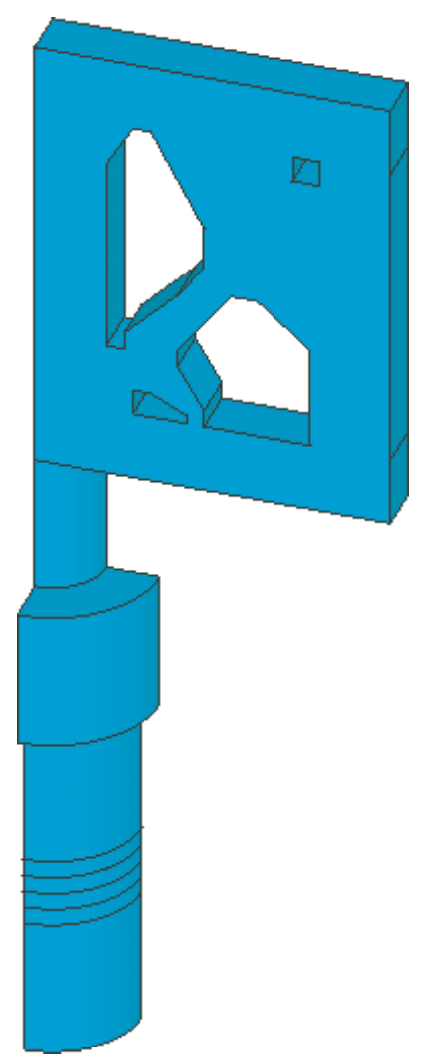

Figura 6.45: Modelo simétrico 3D da estrutura com o sonotrodo otimizado.

em $20 \mathrm{kHz}$, foi alterada no modelo 3D. Foi realizada uma análise harmônica numa faixa de freqüências, entre 19500 e $20500 \mathrm{~Hz}$, para verificar se a freqüência de excitação que apresenta uma uniformização maior que $80 \%$ também alterou em relação à $20 \mathrm{kHz}$. Na Tab. 6.5 são apresentadas as taxas de uniformização de algumas freqüências dentro da 
faixa estipulada.

Tabela 6.5: Taxa de uniformização da superfície de trabalho para algumas freqüências entre 19,5 e $20,5 \mathrm{kHz}$.

\begin{tabular}{c|c}
\hline Freqüência $(k H z)$ & Uniformização $(\%)$ \\
\hline 19,5 & 55,03 \\
19,6 & 64,77 \\
19,7 & 75,23 \\
19,8 & 82,65 \\
19,9 & 82,51 \\
20,0 & 73,52 \\
20,1 & 64,99 \\
20,2 & 57,20 \\
20,3 & 49,99 \\
20,4 & 43,23 \\
20,5 & 36,84 \\
\hline
\end{tabular}

Observa-se pela Tab. 6.5, que na faixa de freqüências entre 19,5 e 20,5 kHz, existe um ponto máximo de uniformização, que está próximo de 19,8 kHz. No entanto, escolhe-se a freqüência de $19,9 \mathrm{kHz}$ que tem a uniformização acima de $80 \%$, e é mais próxima de 20 $k H z$. A deformada da estrutura em 19,9 kHz pode ser vista na Fig. 6.46(a), o modo de pistão $(20728 \mathrm{~Hz})$ na Fig. 6.46(b), o perfil de deslocamentos na superfície de trabalho do sonotrodo no gráfico da Fig. 6.46(c), e o gráfico de impedância na Fig. 6.46(d). Observa-se que neste caso, a impedância resultou em valores altos, o que justifica deslocamentos da ordem de $10^{-8} \mathrm{~m}$. Valores maiores de deslocamentos podem ser obtidos utilizando outras geometrias de amplificadores.

Portanto, a topologia do sonotrodo encontrada atinge todos os objetivos deste trabalho, com a uniformização maior que $80 \%$ (a 19,9 $\mathrm{kHz}$ ) e com a freqüência do modo de pistão maior que $20 \mathrm{kHz}$.

Do mesmo modo que no exemplo 1, a otimização paramétrica pode ser aplicada para modificar as dimensões dos buracos a fim de aumentar o valor da uniformização obtida.

Ainda na tentativa de maximizar a uniformização, observa-se pelos gráficos de convergência dos exemplos 1 e 2, que a mudança nos valores dos limites móveis também influencia na convergência de um valor ótimo de uniformização, podendo existir resultados ainda melhores daqueles apresentados nesta Seção. 


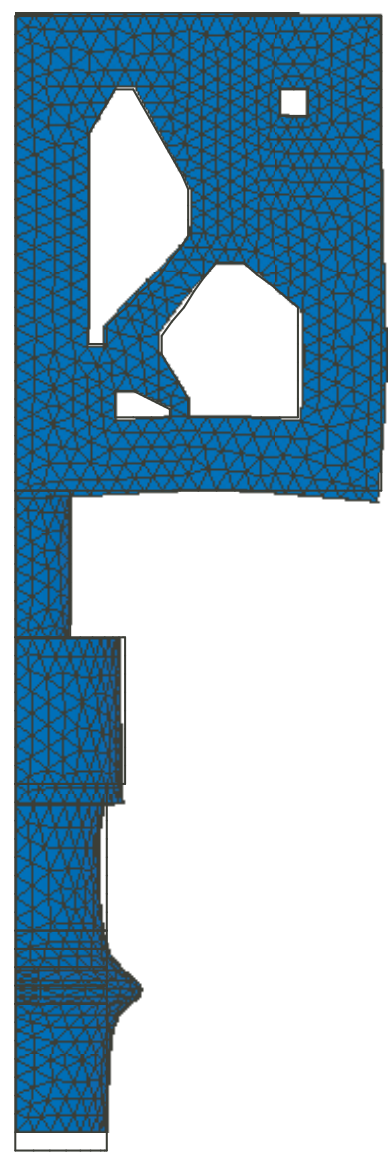

(a) Deformada

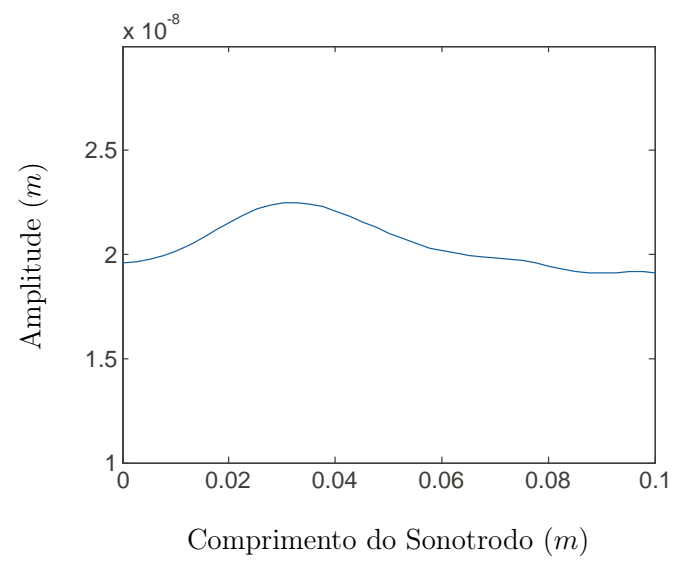

(c)

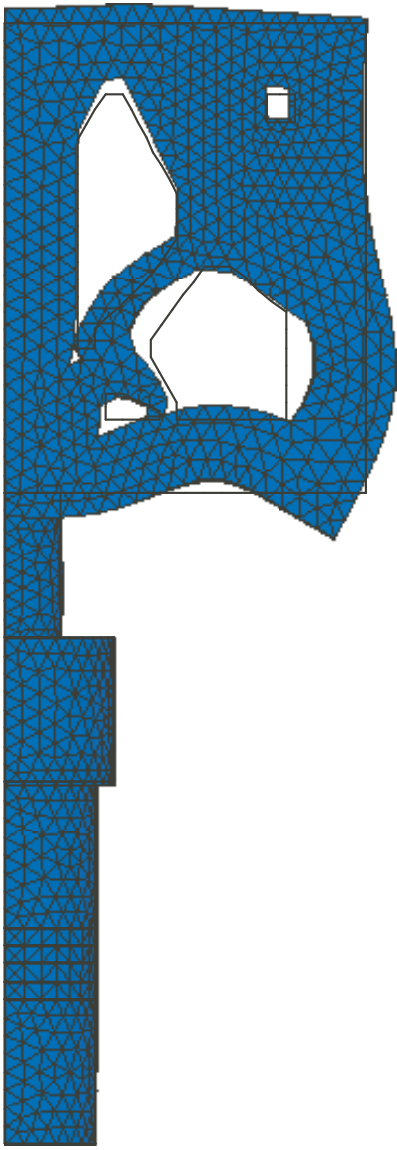

(b) Modo

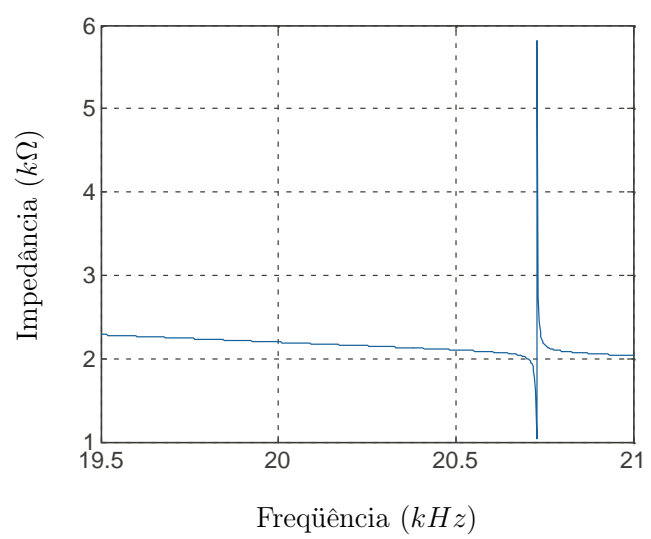

(d)

Figura 6.46: (a) Deformada, (b) modo de pistão, (c) configuração dos deslocamentos e (d) gráfico da impedância do modelo 3D, (ANSYS $\left.{ }^{\circledR}\right)$. 


\section{CONCLUSÕES}

Utilizando o MOT foi desenvolvida uma metodologia sistemática para o projeto do sonotrodo que necessita deslocamentos uniformes em sua face de trabalho.

A formulação para alcançar deslocamentos uniformes é recente e pouco explorada por isso não foi possível ter uma base sólida para esse tipo de formulação, sendo necessário vários testes de implementação para que a formulação apresentada atingisse seu objetivo. Como as funções $L_{1}, L_{2}$ e $L_{4}$ são obtidas utilizando o comportamento dinâmico, a sensibilidade da função multi-objetivo em função dos parâmetros de otimização é muito alta, necessitando de uma extensa análise sobre a influência de todos os parâmetros de otimização nos resultados finais.

Nota-se pelas análises realizadas, que todos os parâmetros de otimização estão interligados entre si, o que aumenta a sensibilidade da função multi-objetivo, dificultando na escolha dos valores desses parâmetros. As análises também revelaram que a rigidez da estrutura pode auxiliar ou atrapalhar na uniformização dos deslocamentos, ou seja, não basta flexibilizar a função multi-objetivo para que ela priorize totalmente a função $L_{1}$ que a uniformização dos deslocamentos será maximizada. Existe uma relação de dependência entre a rigidez e a uniformização de deslocamentos.

$\mathrm{Na}$ modelagem do domínio de projeto com elementos finitos, as formulações de elementos axissimétricos e de EPTM para modelar estruturas rotativas e planas, respectivamente, foram implementadas com sucesso, sendo ambas estendidas para o domínio piezelétrico para modelar os elementos piezelétricos. Comparando os resultados obtidos pelo programa implementado com um software comercial de MEF confiável (ANSYS ${ }^{\circledR}$ ), os erros apresentados foram menores que $0,1 \%$, comprovando a eficácia e precisão dos modelos implementados. A comparação feita entre a análise Tridimensional (3D) e a Bidimensional (2D) revelou que o modelo proposto em $2 \mathrm{D}$ pode ser utilizado como simplificação do domínio em 3D, com erros menores que 1\% (Tabela 6.4), exceto pelo primeiro modo de vibração, que no entanto, neste trabalho pode ser descartado, pois somente os modos próximos a $20 \mathrm{kHz}$ são de interesse. 
O estudo aprofundado feito neste trabalho sobre a influência dos modelos de material SIMP e RAMP, e dos diferentes valores para penalização na geração das escalas de cinza, mostrou que utilizando SIMP na densidade e na rigidez, e fazendo com que a penalização da densidade varie de 2 a 10 e a penalização do módulo de elasticidade varie de 1 a 5 , as escalas de cinza são minimizadas, reduzindo também os modos localizados.

Uma das grandes dificuldades deste trabalho foi o cálculo feito para obter as freqüências de ressonância e modos de vibração na análise modal. Pelo fato de estarmos trabalhando com elementos piezelétricos, foi necessário adaptar a matriz de massa do elemento finito para que fosse possível realizar a análise modal utilizando a função "eigs" do MatLab ${ }^{\circledR}$, baseada na rotina ARPACK. Porém, existem poucas referências na literatura que tratam a respeito dessas adaptações necessárias para o cálculo de autovalores e autovetores piezelétricos. Algumas referências explicitam alguns tipos de modificações mais simples, porém, possuem custo computacional muito alto, necessitando de muito espaço em memória e tempo computacional. A modificação apresentada neste trabalho permitiu a redução do custo computacional em relação à essas modificações mais simples, fornecendo resultados precisos e confiáveis.

Os exemplos apresentados na seção de "Resultados" mostram que é possível obter a topologia ótima que possua uniformização dos deslocamentos maior que $80 \%$ na face de trabalho do sonotrodo, mantendo o modo de pistão acima da freqüência limite audível pelo homem, de $20 \mathrm{kHz}$. Contudo, por ser um problema dinâmico, apesar de utilizar penalizações altas para os modelos de material, os resultados ainda estão sujeitos à escala de cinza, o que prejudica a etapa de pós-processamento, onde a escala de cinza é substituída por material ou vazio, a critério do projetista.

Portanto, foi observado neste trabalho que as formulações propostas mostraram-se eficientes e sistemáticas para o projeto de sonotrodos com deslocamentos uniformes na sua face de trabalho, empregados em transdutores piezelétricos que operam em freqüências ultra-sônicas.

\subsection{Proposta de Trabalhos Futuros}

Como já mencionado na seção 6.5, uma possível tentativa para aumentar os valores da uniformização seria, após as etapas de pós-processamento apresentadas, submeter o resultado à otimização paramétrica, onde as dimensões dos furos podem ser modificadas para apresentar valores maiores de uniformização. Pode-se também, modificar o domínio 
inicial de projeto, mas no entanto, não é possível garantir que isso forneça um mínimo local que apresente melhores resultados.

Como apresentado nos exemplos 1 e 2 do capítulo de resultados, embora a uniformização tenha atingido valores aceitáveis, os deslocamentos atingiram valores muito baixos (da ordem de $10^{-7} \mathrm{~m}$ ), o que é insuficiente para aplicações como por exemplo, de corte e solda ultrassônico de tecidos. Então, para ser possível a obtenção de deslocamentos com amplitudes maiores na superfície de trabalho, pode-se considerar trabalhar com os modos de vibrar da estrutura, que neste caso, seria o modo piezelétrico (ou modo de pistão, como foi chamado neste trabalho). Assim, dado que o modo é encontrado pela formulação MAC, ele é submetido à otimização para que os deslocamentos na superfície de trabalho sejam uniformizados. Mas trabalhar com modos de vibrar é mais complicado, já que os valores dos deslocamentos no modo calculados pela rotina de autovalor devem ser normalizados, pois numericamente, os deslocamentos tendem ao infinito na ressonância, o que também, aumenta a sensibilidade da função objetivo, uma vez que os valores encontrados são muito altos. Nesse caso seria necessário utilizar uma abordagem que trabalha com o modo de vibrar (autovetor) obtido na analise modal (MAEDA et al., 2006).

$\mathrm{Na}$ tentativa de se melhorar a convergência da função multi-objetivo, pode-se aplicar valores de limites móveis independentes para cada variável de projeto. Por exemplo, os valores dos limites móveis associados a cada variável de projeto podem ser estabelecidos de acordo com o gradiente da função multi-objetivo referente àquela variável de projeto em questão. Ou seja, se o gradiente for muito alto, quer dizer que a função é bastante sensível àquela variável, então seus limites serão menores.

A formulação proposta neste trabalho também pode ser aplicada para sonotrodos cilíndricos, mas para isso, há necessidade da inclusão de restrições de manufatura de modo a não existirem furos no resultado, pois o domínio plano, neste caso, simularia uma estrutura cilíndrica axissimétrica, impossibilitando sua fabricação, a não ser que a mesma seja construída em pedaços e depois montada, o que modificaria os resultados obtidos.

A restrição de manufatura pode ser aplicada também em sonotrodos do tipo bloco ou lâmina-larga, controlando a forma e a quantidade de furos ou a forma do contorno do sonotrodo. Como exemplo, pode ser incluída uma restrição onde os furos devem ser parecidos com os furos passantes dos sonotrodos bloco e lâmina-larga atuais, de modo a modificar a posição e dimensões dos furos.

Para melhorar os resultados e as etapas de pós-processamento, discretizações maiores podem ser utilizadas. Contudo, isso não foi possível até a finalização deste trabalho 
pela limitação apresentada pelo sistema operacional utilizado, o Windows ${ }^{\circledR}$ de $32 \mathrm{bits}$, o qual reconhece apenas $3 \mathrm{~GB}$ de memória RAM (somando memória física e virtual), e não permitiu que o software MatLab ${ }^{\circledR}$ utilizasse toda a capacidade. A solução seria rodar o programa num sistema operacional de 64bits (Windows ou Linux), pois esses sistemas não possuem limitações de memória.

Pode-se também, considerar a otimização topológica no modelo em três dimensões completo, sem simetrias. Assim, não seriam desprezados os modos não-simétricos da estrutura e o acoplamento do modelo axissimétrico com o plano, porém seria necessário uma quantidade muito maior de memória RAM, pois o custo computacional de modelos em 3D é muito alto. 


\section{Anexo A - Verificação da Generalidade da Formulação Proposta quanto à Uniformização dos Deslocamentos}

Neste anexo são apresentados os resultados de dois exemplos para verificar a eficiência e generalidade da formulação proposta e do programa implementado para diferentes freqüências de excitação, como por exemplo, 3 e $10 \mathrm{kHz}$.

\section{A.1 Freqüência de Excitação igual a $3 \mathbf{k H z}$}

Como a freqüência de excitação é menor, a sensibilidade do problema em relação às variáveis de projeto será menor, comparado com a operação em $20 \mathrm{kHz}$, possibilitando que o domínio de projeto seja modificado (Fig. A.1) de modo a aumentar a área otimizável onde o MOT irá distribuir material. Com isso, existe a possibilidade de se encontrar um mínimo local apresentando melhores resultados do que aqueles utilizando um domínio mais fechado.

Alguns parâmetros de otimização também foram modificados para se adaptarem ao novo problema. Os valores são $w_{1}=300, w_{2}=20, w_{3}=450, w_{4}=0, d_{0}=0,45$, $\Theta_{\text {lim_sup }}=0,55 \%, \beta=0,01 \mu \mathrm{m}$ e $R_{\max }=0,0055 \mathrm{~mm}$. O parâmetro $w_{4}$ é igual a zero pois deseja-se verificar a eficiência e generalidade da formulação quanto à uniformização de deslocamentos, e o modo de pistão será seguido somente para verificação. A otimização termina quando completadas 100 iterações, a penalização da densidade $q$ inicia em 2 e aumenta em duas unidades na iterações 50, e a penalização do módulo de elasticidade $p$ inicia em 1 e aumenta em uma unidade também na iteração 50.

Assim, a Fig. A.2 apresenta a topologia ótima obtida pelo MOT, sua deformada (apenas o sonotrodo) e o seu modo de pistão.

Na Fig. A.3 são apresentados os gráficos da função multi-objetivo, $L_{1}, L_{2}$ e $L_{3}$ para 


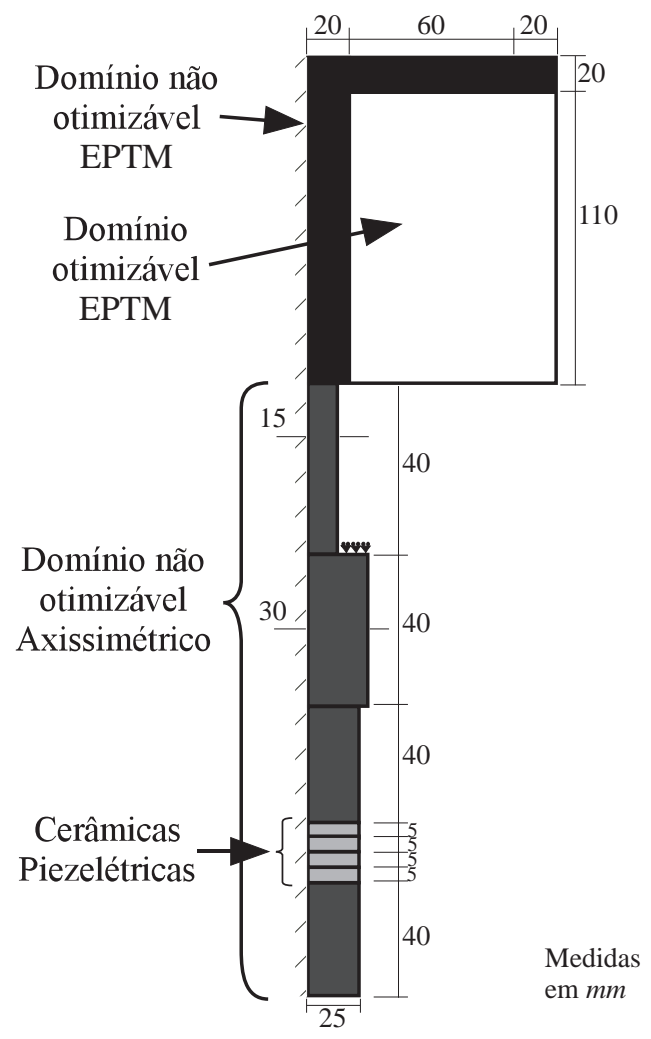

Figura A.1: Domínio de projeto.

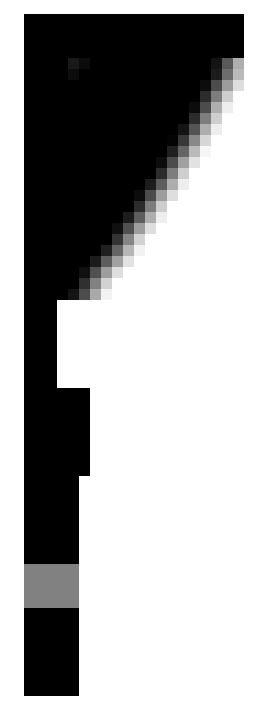

(a)

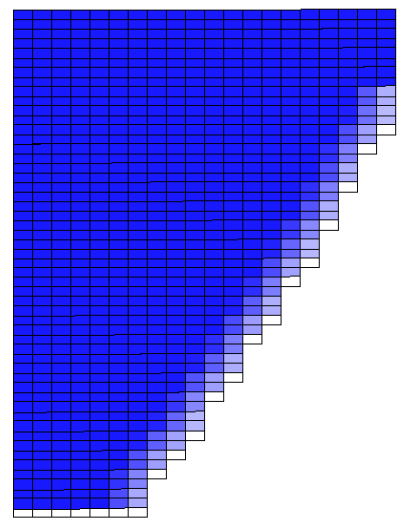

(b)

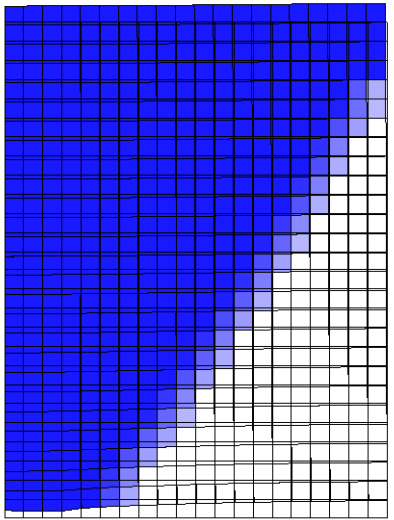

(c)

Figura A.2: (a) Topologia ótima, (b) deformada somente do sonotrodo da topologia ótima a $3 k H z$, e (c) modo de pistão a $4,5 k H z$.

mostrar que a convergência está sendo alcançada, ou seja, foram minimizadas as funções multi-objetivo, $L_{1}$ e $L_{3}$, e a função $L_{2}$ foi maximizada, apesar dos degraus apresentados nos gráficos causados pelas mudanças nas penalizações. O gráfico da função $L_{4}$ não foi 


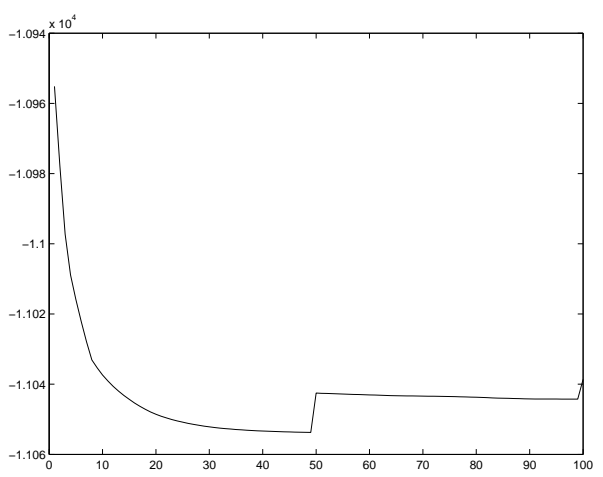

(a) Função multi-objetivo

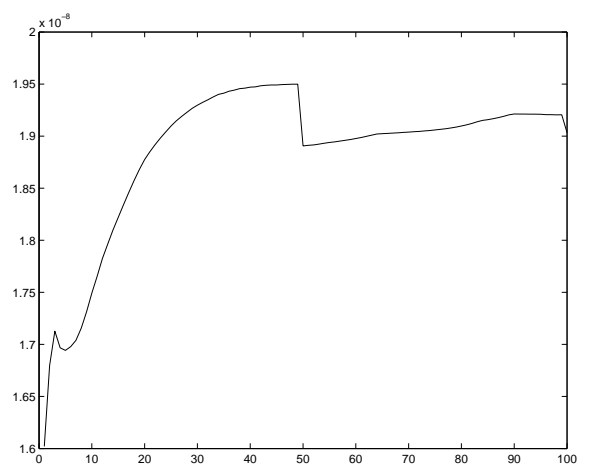

(c) Função $L_{2}$

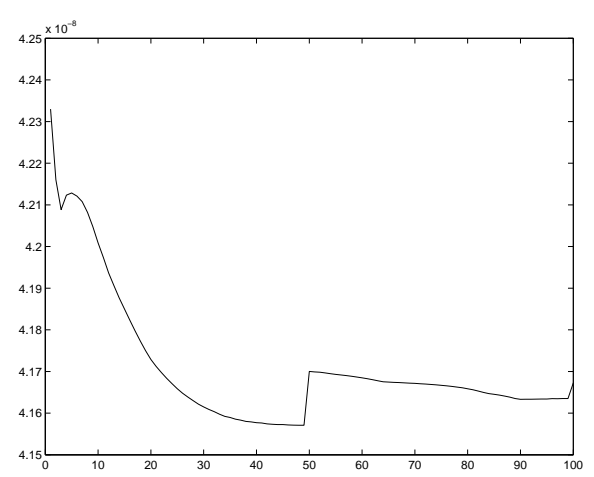

(b) Função $L_{1}$

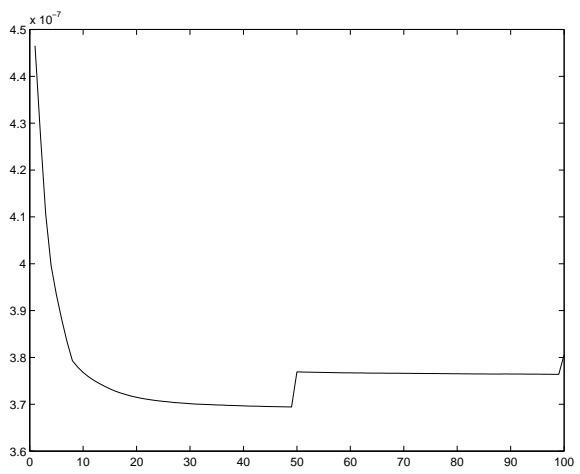

(d) Função $L_{3}$

Figura A.3: Gráfico de convergência das funções. A abscissa dos gráficos representam o número de iterações e a ordenada os valores das respectivas funções.

apresentado pois ela não foi considerada neste exemplo.

Para mostrar a uniformização obtida, são apresentados os gráficos da configuração de deslocamentos na face superior do sonotrodo (Fig. A.4(a)) e o gráfico de uniformização (Fig. A.4(b)). O gráfico de convergência da freqüência do modo de pistão é apresentado na Fig. A.4(c).

Ao final da otimização, a razão de uniformização $U_{\min } / U_{\max }$, em $3 \mathrm{kHz}$, e a freqüência do modo de pistão (primeiro modo de vibrar da estrutura) resultaram em valores iguais a $93,48 \%$ e $4472 \mathrm{~Hz}$, respectivamente.

Observa-se que a freqüência do modo de pistão final (aquele que possui em fase todas as amplitudes da face de trabalho do sonotrodo) ficou próxima à freqüência de excitação do problema, mesmo com a função $L_{4}$ não estar atuando. Isso era de se esperar, pois 3 $k H z$ é uma freqüência próxima do modo de operação quase-estático do transdutor, que é similar a um modo de pistão. 


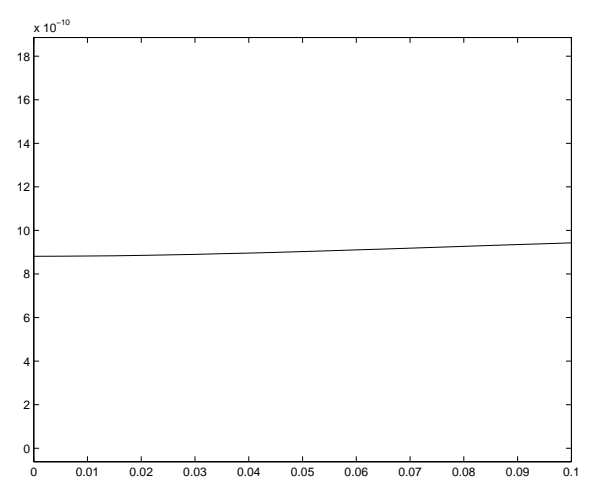

(a) Configuração dos deslocamentos

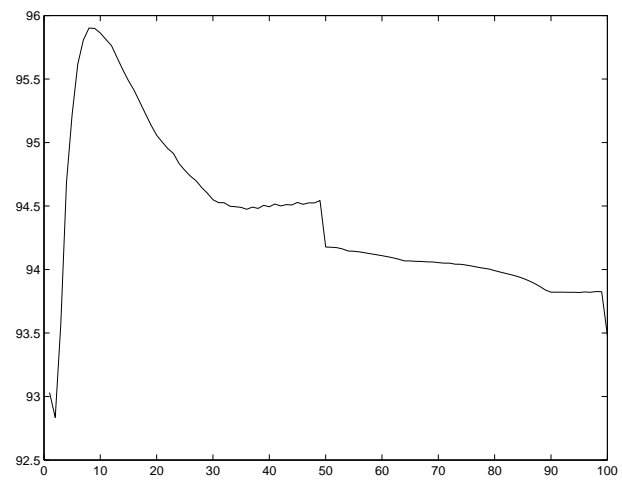

(b) Uniformização $\left(U_{\min } / U_{\max }\right)$

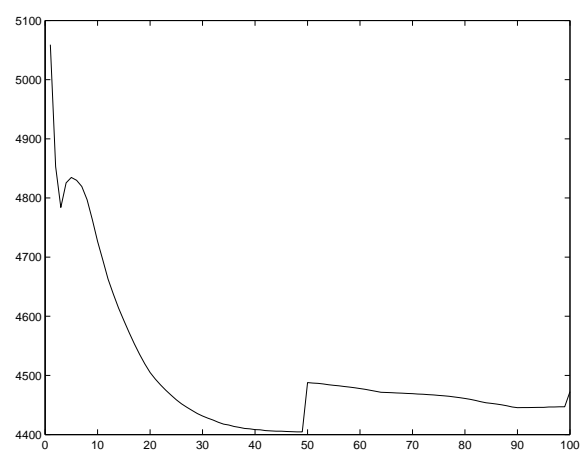

(c) Freqüência do modo de pistão

Figura A.4: Gráficos de uniformização e de freqüência. A abscissa dos gráficos representam o número de iterações e a ordenada os valores das respectivas funções.

No entanto, apesar da uniformização ser igual a 93,48\%, a amplitude dos deslocamentos na face do sonotrodo resultaram em valores inferiores a $1 \mathrm{~nm}$, como pode ser visto na Fig. A.4(a). Isso pode ser explicado pelo fato da impedância do conjunto, a $3 \mathrm{kHz}$, ser muito alta (aproximadamente 3,9 $\mathrm{k} \Omega$ ), como pode ser observado no gráfico da Fig. A.5. Observa-se também neste gráfico, que a freqüência de ressonância (oscilação para baixo na curva) e anti-ressonância (oscilação para cima na curva) são praticamente iguais, o que significa que este modo não é fortemente acoplado.

Portanto, a formulação apresentada neste trabalho pode ser utilizada também para a freqüência de excitação igual a $3 \mathrm{kHz}$, sendo necessário modificações nos parâmetros de otimização e no domínio inicial de projeto. 


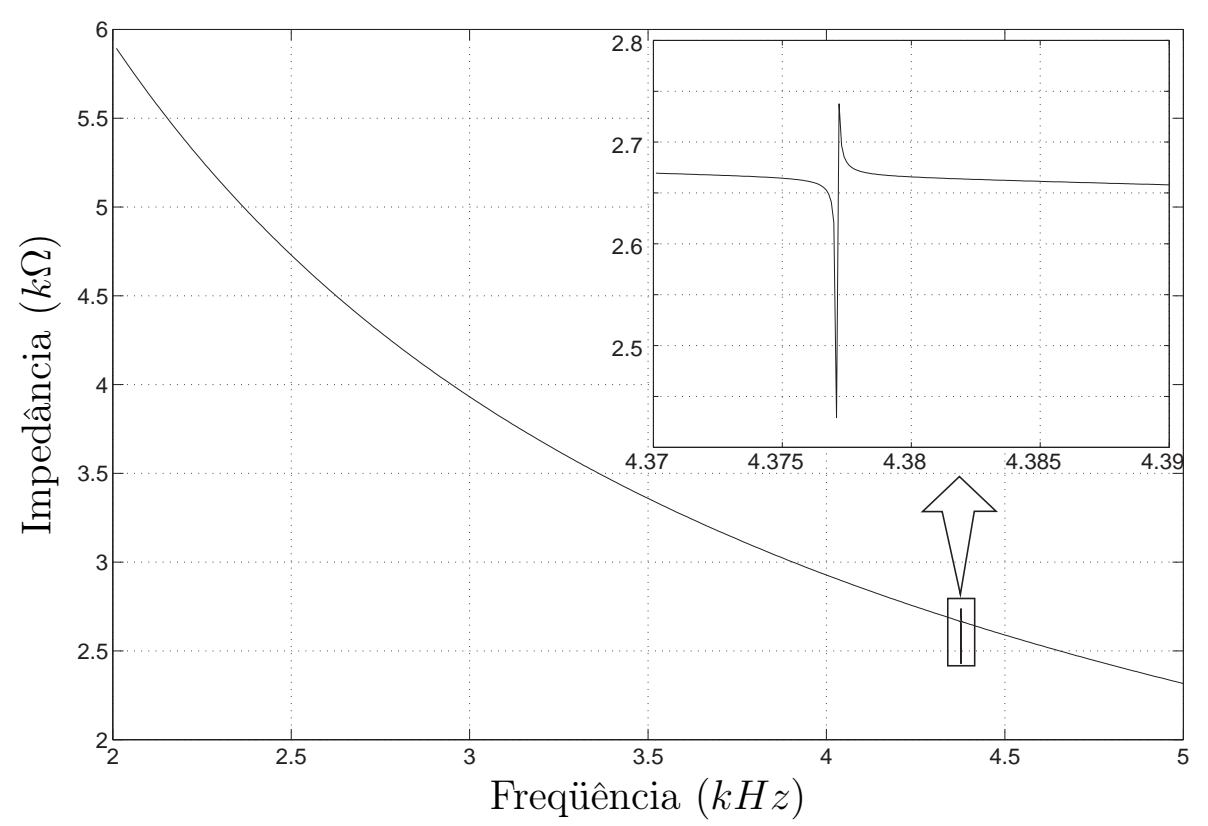

Figura A.5: Gráfico da Impedância próximo à freqüência de $3 \mathrm{kHz}$.

\section{A.2 Freqüência de Excitação igual a $10 \mathrm{kHz}$}

Para este exemplo foi utilizado o mesmo domínio daquele utilizado para $20 \mathrm{kHz}$, apresentado na Fig. 6.7(a).

Alguns parâmetros de otimização foram novamente modificados para se adaptarem ao novo problema. Os valores são $w_{1}=300, w_{2}=30, w_{3}=400, w_{4}=0, d_{0}=0,45$, $\Theta_{\text {lim_sup }}=0,55 \%, \beta=0,073 \mu \mathrm{m}$ e $R_{\max }=0,0055 \mathrm{~mm}$. Novamente, o parâmetro $w_{4}$ é igual a zero e o modo de pistão também será seguido somente para verificação. A otimização termina quando completadas 300 iterações, a penalização da densidade $(q)$ no modelo de material inicia com o valor 2, e aumenta em 2 unidades nas iterações 60 e 120, e a penalização do módulo de elasticidade $(p)$ inicia com o valor 1 , e aumenta em meia unidade $(0,5)$ nas iterações 30, 60, 90 e 120. Ao final da otimização, $q=3$ e $p=6$.

Assim, a Fig. A.6 apresenta a topologia ótima obtida pelo MOT, sua deformada (apenas o sonotrodo) e o seu modo de pistão.

Na Fig. A.7 são apresentados os gráficos da função multi-objetivo, $L_{1}, L_{2}$ e $L_{3}$ para mostrar a convergência de cada função. Foram minimizadas as funções multi-objetivo, $L_{1}$ e $L_{2}$, e a função $L_{3}$ foi maximizada. No entanto, a função $L_{2}$ deveria ser maximizada e a função $L_{3}$, minimizada. O que ocorreu neste caso é que a solução atingiu um mínimo local, que priorizou somente somente a função $L_{1}$. O gráfico da função $L_{4}$ não foi apresentado pois ela também não foi considerada neste exemplo. 


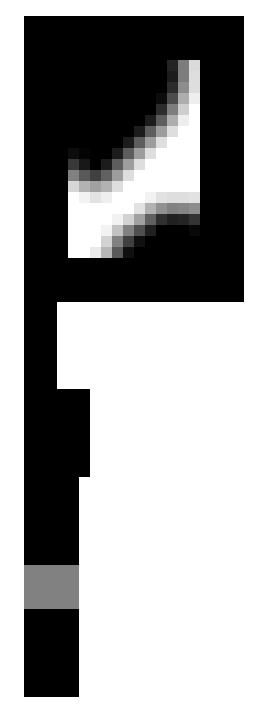

(a)

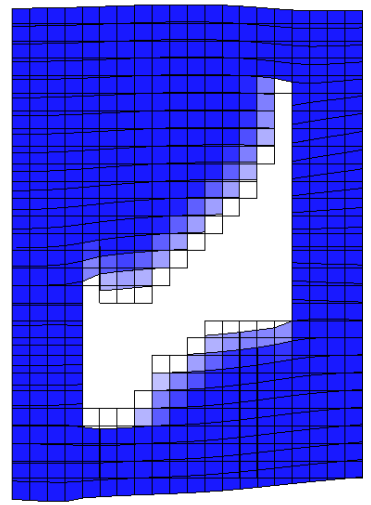

(b)

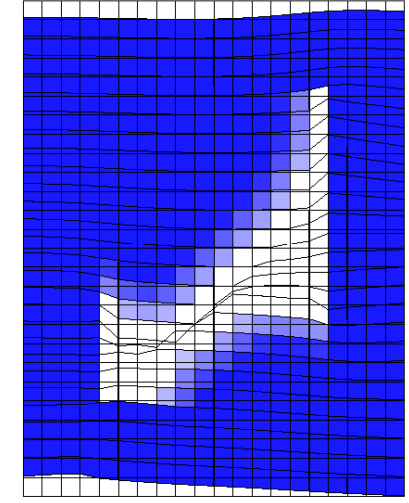

(c)

Figura A.6: (a) Topologia ótima, (b) deformada somente do sonotrodo da topologia ótima a $10 \mathrm{kHz}$, e (c) modo de pistão a $9,8 \mathrm{kHz}$.

Então, para mostrar a uniformização obtida, são apresentados os gráficos da configuração de deslocamentos na face superior do sonotrodo (Fig. A.8(a)) e o gráfico de uniformização (Fig. A.8(b)). O gráfico de convergência da freqüência do modo de pistão é apresentado na Fig. A.8(c).

Ao final da otimização, a razão de uniformização $U_{\min } / U_{\max }$, em $10 \mathrm{kHz}$, e a freqüência do modo de pistão (quarto modo de vibrar da estrutura) resultaram em valores iguais a $66,46 \%$ e $9790 \mathrm{~Hz}$, respectivamente.

Nota-se que a uniformização é menor que 80\%, o que também pode ser explicado pelo fato de que a solução atingiu um mínimo local, que embora tenha priorizado somente a função $L_{1}$, não foi capaz de aumentar a uniformização para níveis aceitáveis. No entanto o gráfico da Fig. A.8(b) mostra que a uniformização foi maximizada, tornando possível que a uniformização atinja o valor mínimo de $80 \%$, mediante a escolha certa do domínio inicial e dos parâmetros de otimização. Quanto à freqüência, observa-se novamente que a freqüência do modo de pistão final ficou próxima à freqüência de excitação do problema, pois $10 \mathrm{kHz}$ é uma freqüência próxima do modo de operação quase-estático do transdutor, que é similar a um modo de pistão.

Levantando a curva de impedância para este caso (Fig. A.9), nota-se que a freqüência de excitação utilizada $(10 \mathrm{kHz})$ está próxima à uma freqüência de ressonância, cujo modo é similar ao modo de pistão. 


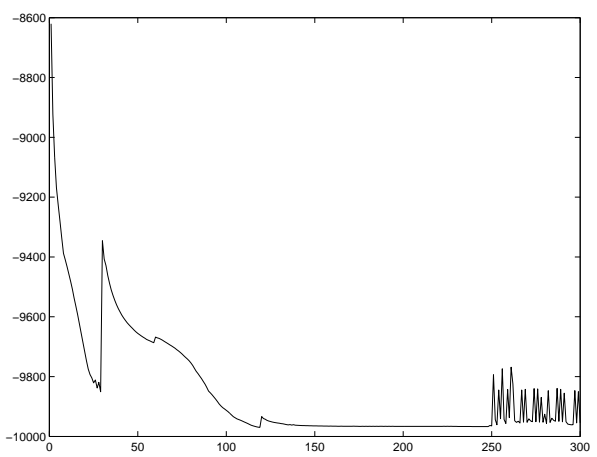

(a) Função multi-objetivo

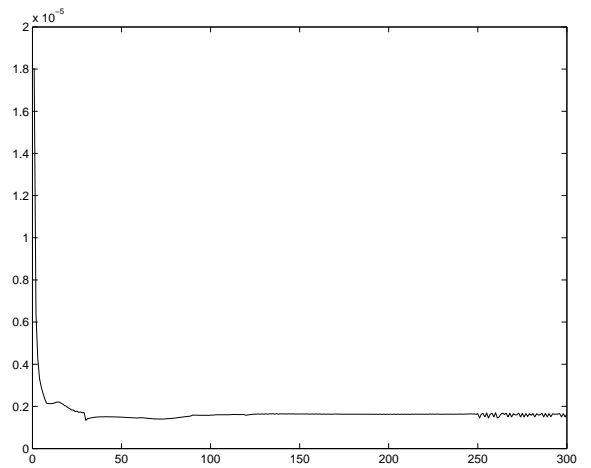

(c) Função $L_{2}$

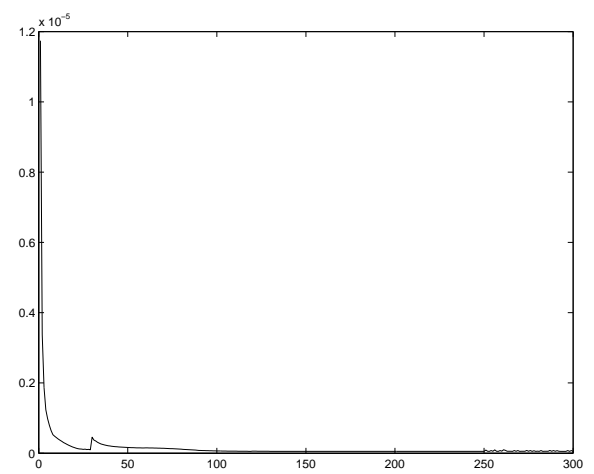

(b) Função $L_{1}$

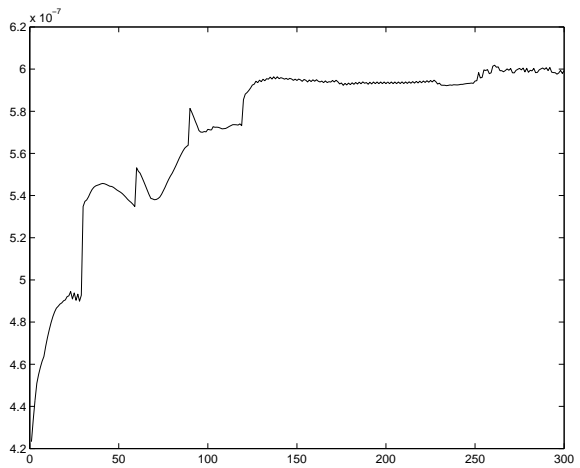

(d) Função $L_{3}$

Figura A.7: Gráfico de convergência das funções. A abscissa dos gráficos representam o número de iterações e a ordenada os valores das respectivas funções.

Portanto, mediante os exemplos apresentados, pode-se concluir que a formulação apresentada neste trabalho pode ser utilizada para diferentes freqüências de excitação com o objetivo de uniformizar as amplitudes na face de trabalho do sonotrodo, sendo necessário modificações nos parâmetros de otimização e no domínio inicial de projeto para cada caso. 


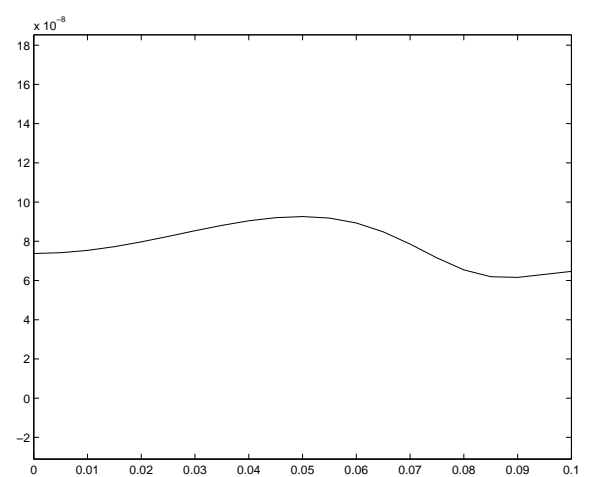

(a) Configuração dos deslocamentos

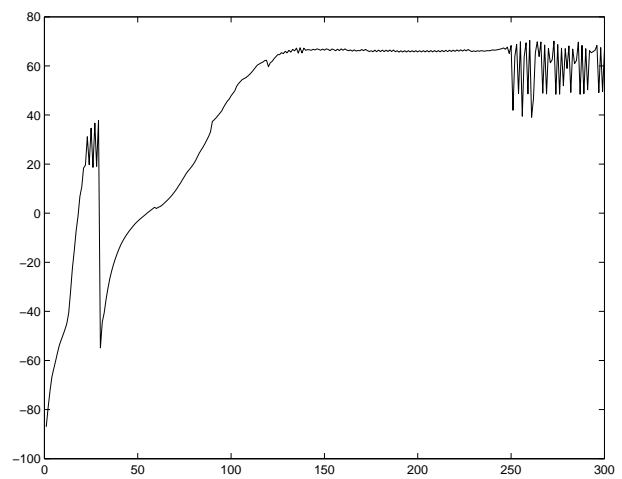

(b) Uniformização $\left(U_{\min } / U_{\max }\right)$

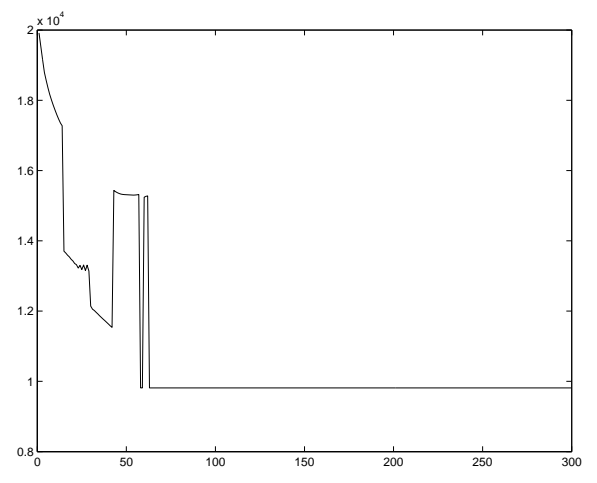

(c) Freqüência do modo de pistão

Figura A.8: Gráficos de uniformização e de freqüência. A abscissa dos gráficos representam o número de iterações e a ordenada os valores das respectivas funções.

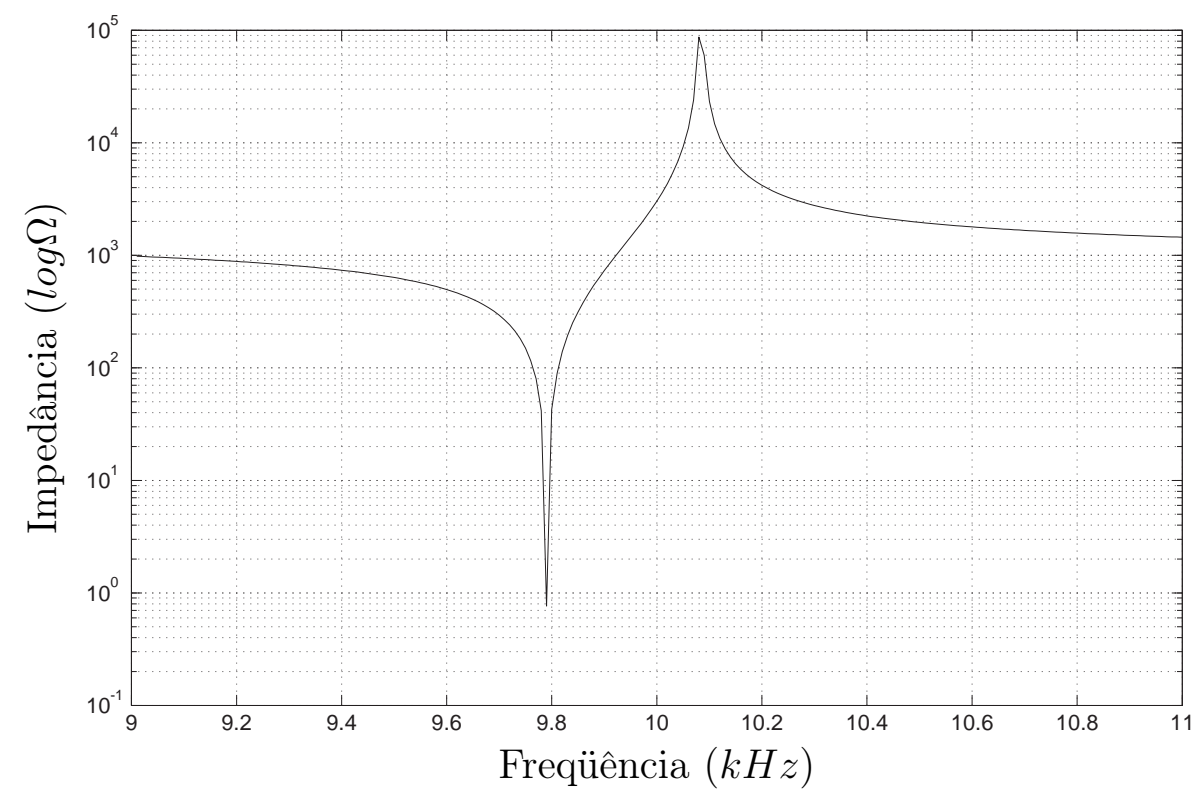

Figura A.9: Gráfico da Impedância próximo à freqüência de $10 \mathrm{kHz}$. 


\section{Anexo B - Comparação 2D \3D}

Ao utilizar um modelo 2D como aproximação do modelo 3D, de modo a reduzir o custo computacional, é necessário considerar, no caso deste trabalho, que se está aproximando o acoplamento entre um cilindro e um bloco (amplificador e sonotrodo respectivamente, Fig. B.1(a)) por um plano (Fig. B.1(c)), bem como considerar que se está desprezando modos de vibrar em outros planos da estrutura e modos não-simétricos. A análise feita do acoplamento entre o amplificador e o sonotrodo utilizou o domínio simétrico da estrutura (um quarto da estrutura completa, Fig. B.1(c)) para reduzir o custo computacional (não comprometendo a configuração dos deslocmentos gerados), e a análise modal foi feita utilizando o modelo 3D completo (Fig. B.1(a)) para que sejam verificadas algumas freqüências e modos não-simétricos da estrutura.

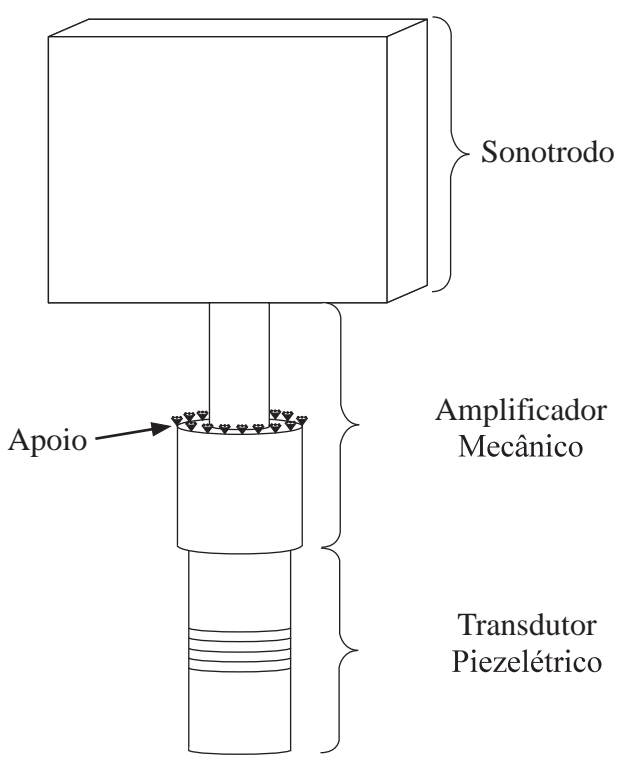

(a)

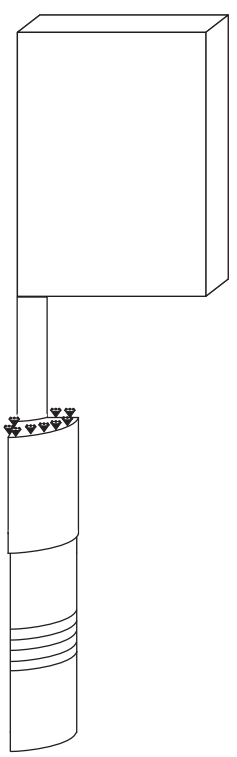

(b)

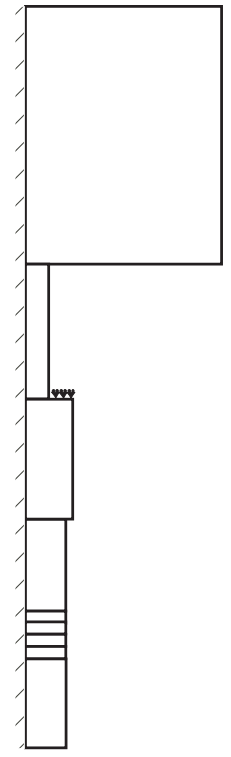

(c)

Figura B.1: (a) Modelo 3D completo, (b) simétrico e (c) 2D simétrico. 


\section{B.1 Acoplamento Amplificador/Sonotrodo}

A seguir, tem-se as figuras das deformadas do modelo simétrico 3D nas vistas isométrica e frontal, e do modelo simétrico 2D, ambos excitados em $20 \mathrm{kHz}$.

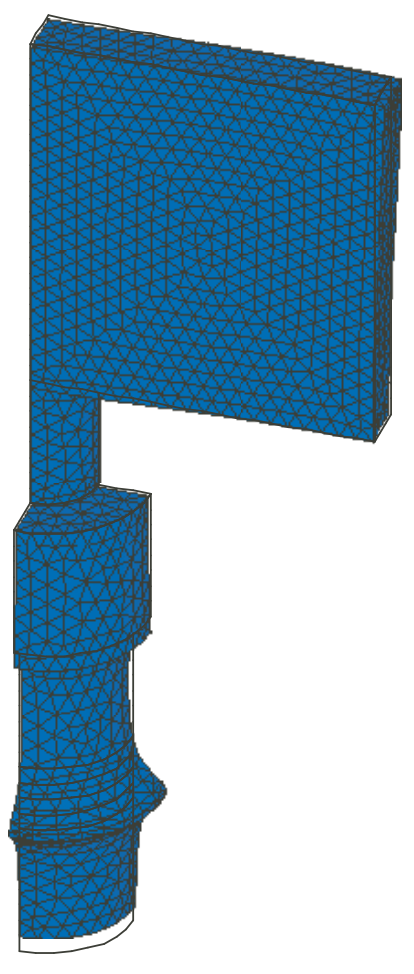

(a)

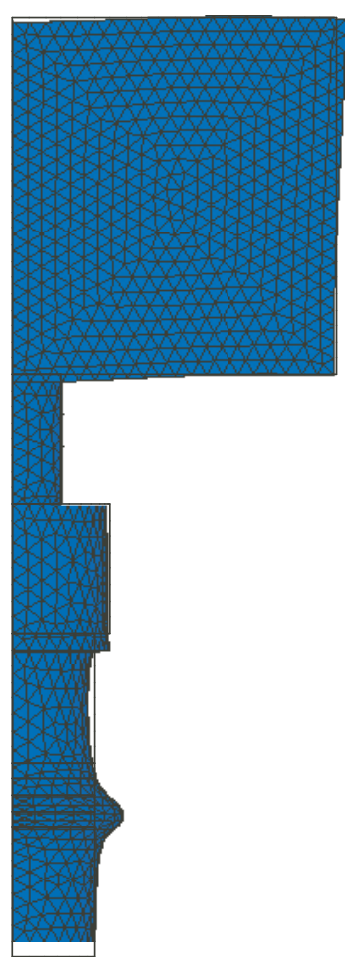

(b)

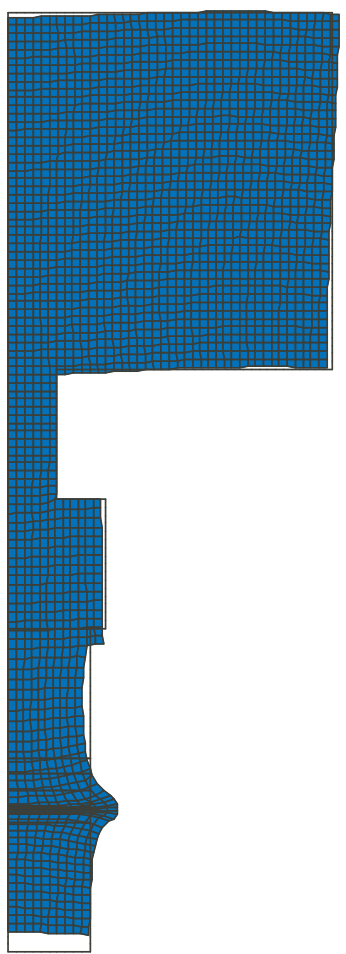

(c)

Figura B.2: Deformadas da estrutura excitado em $20 \mathrm{kHz}$ do modelo 3D em (a) Vista isométrica, (b) frontal e (c) do modelo 2D simétrico.

De acordo com as deformadas, nota-se que a configuração dos deslocamentos gerados pelo transdutor numa freqüência de $20 \mathrm{kHz}$ é muito semelhante nos modelos 2D e 3D. No entanto, não se pode concluir que são iguais, pois as amplitudes de deslocamento podem ser diferentes. Assim, plota-se na Fig. B.3, o gráfico dos deslocamentos tanto no acoplamento entre o amplificador e o sonotrodo (que é o caso mais crítico de aproximação), quanto na superfície de trabalho do sonotrodo. No caso do modelo 3D, a curva se refere ao deslocamento no plano de simetria, ou seja, na parte de trás do modelo da Fig. B.2(a).

Analisando os gráficos, a diferença média das amplitudes de deslocamento no acoplamento amplificador/sonotrodo entre os modelos 3D e 2D é igual a 3,57\%, e na superfície de trabalho do sonotrodo, essa diferença média é igual a 8,7\%. Então, essa comparação entre os modelos 3D e 2D mostrou que a aproximação 2D pode ser utilizada sem comprometer os resultados. No entanto, deve-se estar ciente de que uma análise 


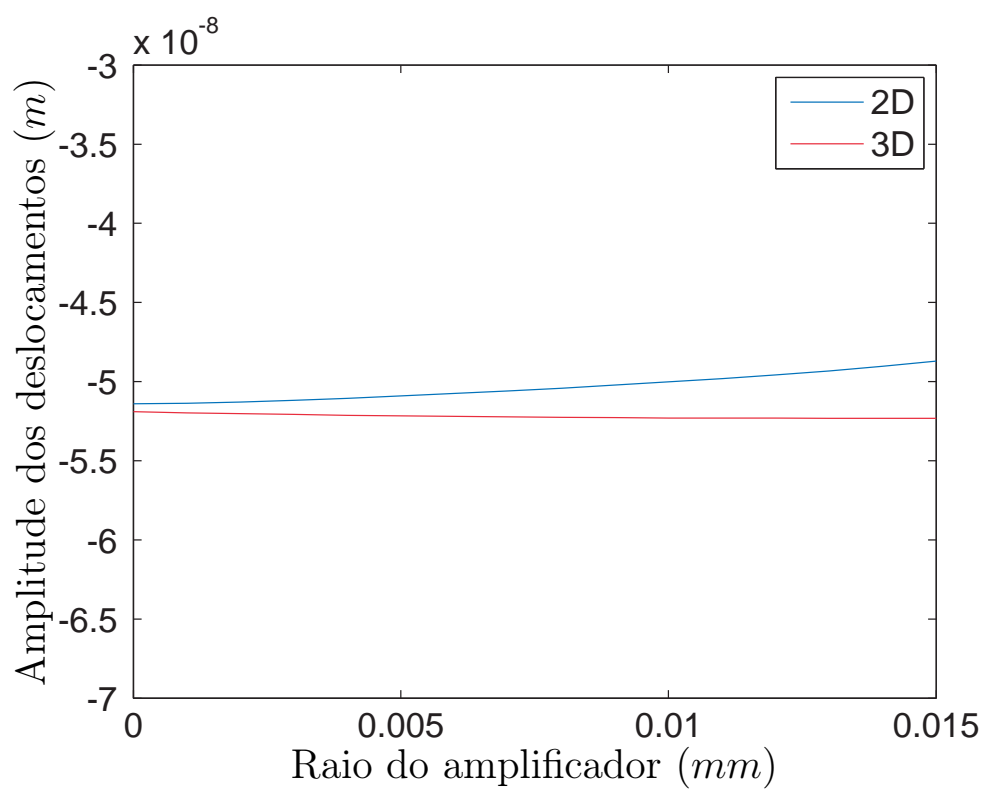

(a) Acoplamento Amplificador/Sonotrodo

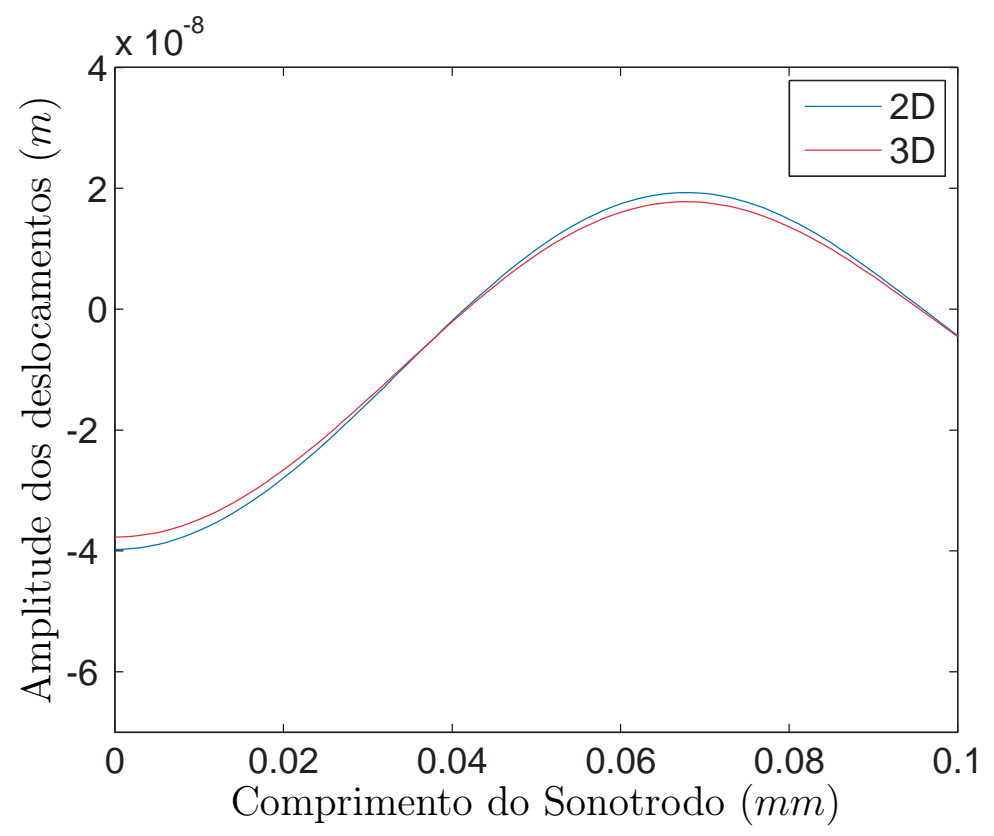

(b) Superfície de trabalho do sonotrodo

Figura B.3: Gráficos das amplitudes de deslocamento dos modelos 3D e 2D, (a) no acoplamento entre amplificador e sonotrodo e, (b) na superfície superior do sonotrodo.

do modelo 3D fornece resultados muito mais próximos do real, mas com um custo computacional muito maior. 


\section{B.2 Análise modal do modelo 3D completo}

Para verificar os modos não-simétricos e os modos em outros planos da estrutura, foram calculados os modos de vibrar dos modelos 3D completo (Fig. B.1(a)), 3D simétrico(Fig. B.1(b)) e 2D simétrico (Fig. B.1(c)) entre 18 e 22 kHz. Na Tab. B.1 são apresentados o número de modos de cada modelo e as freqüências de ressonância associadas a cada modo.

Tabela B.1: Análise modal dos modelos 3D completo, 3D simétrico e 2D simétrico entre 18000 e $22000 \mathrm{~Hz}$.

\begin{tabular}{c|c|c|c}
\hline Modo & 3D completo $(H z)$ & 3D simétrico $(H z)$ & 2D simétrico $(H z)$ \\
\hline 1 & 18835 & - & - \\
2 & 19378 & - & - \\
3 & 20191 & 20191 & 20246 \\
4 & 20582 & 20582 & 20582 \\
5 & 20706 & - & - \\
6 & 20913 & - & - \\
7 & 21337 & - & - \\
8 & 21341 & - & - \\
9 & 21436 & - & - \\
10 & 21855 & - & - \\
\hline
\end{tabular}

Nota-se que, numa faixa de $4 k H z$, perdem-se 8 modos ao utilizar a simetria em 3D ou a aproximação 2D. Esses modos são os modos não-simétricos ou modos em outros planos da estrutura. Os dois modos encontrados nas análises dos modelos 3D simétrico e 2D simétrico correspondem ao terceiro e quarto modos, respectivamente, e a Fig. B.4 mostra suas deformadas. Já a Fig. B.5 mostra alguns exemplos dos modos não-simétricos e em outros planos. 

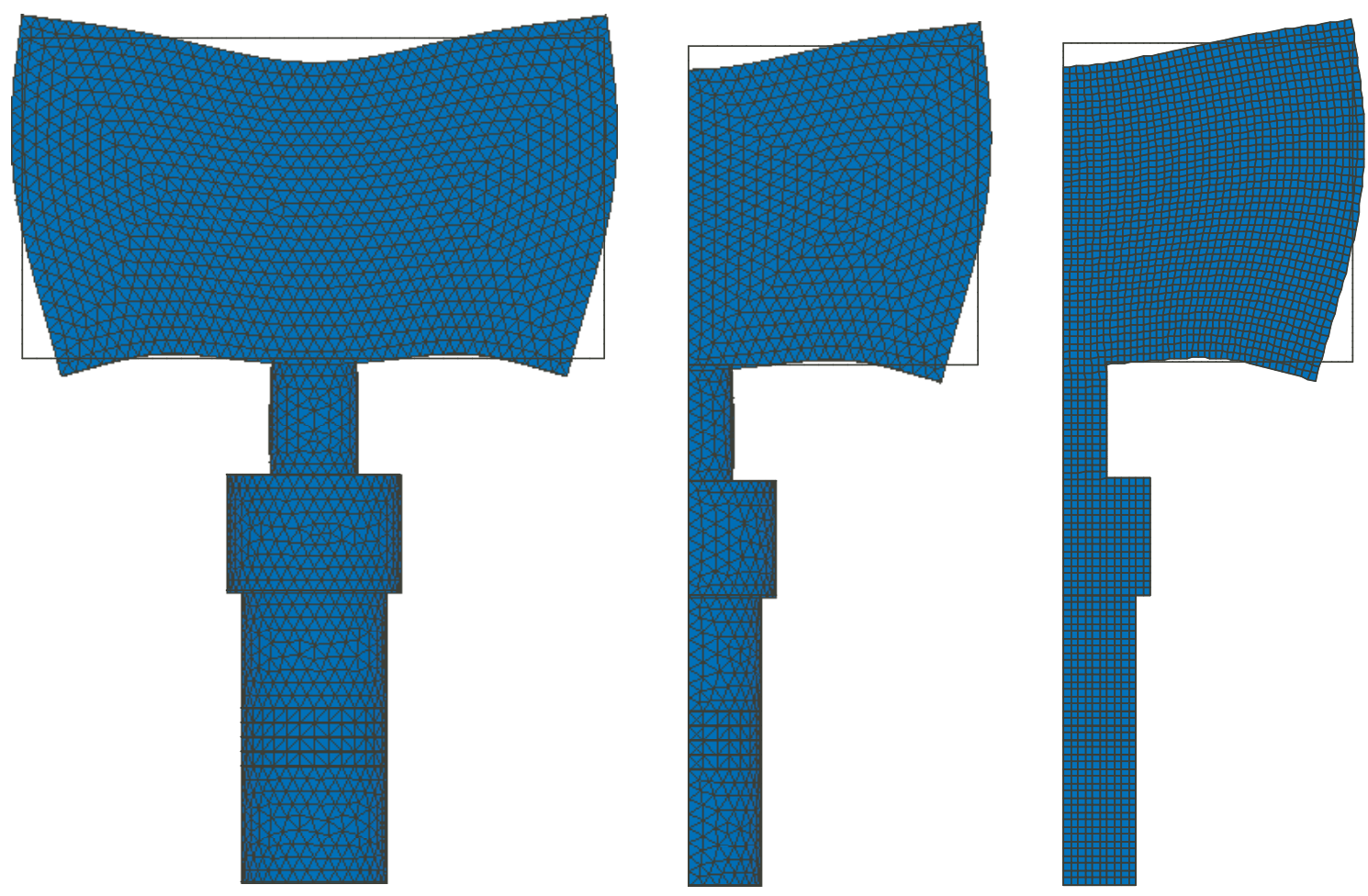

(a) Modo 3
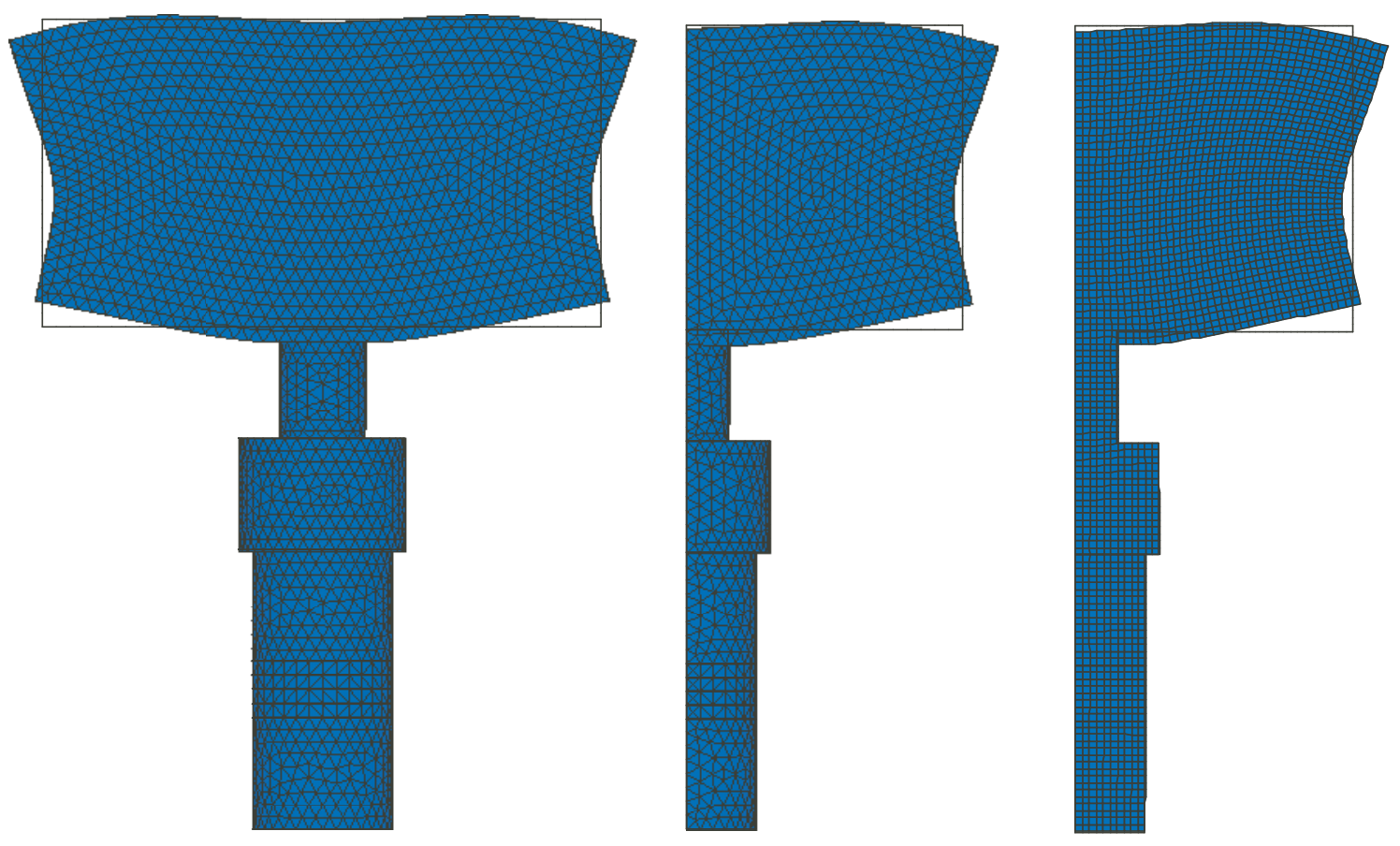

(b) Modo 4

Figura B.4: Terceiro e quarto modos do modelo 3D completo, correspondentes aos modos dos modelos 3D simétrico e 2D simétrico. 


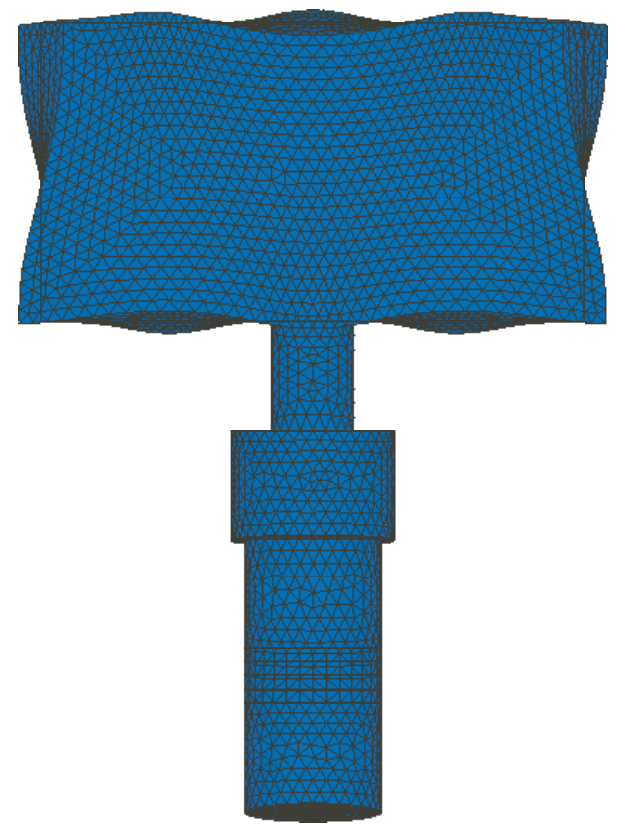

(a)

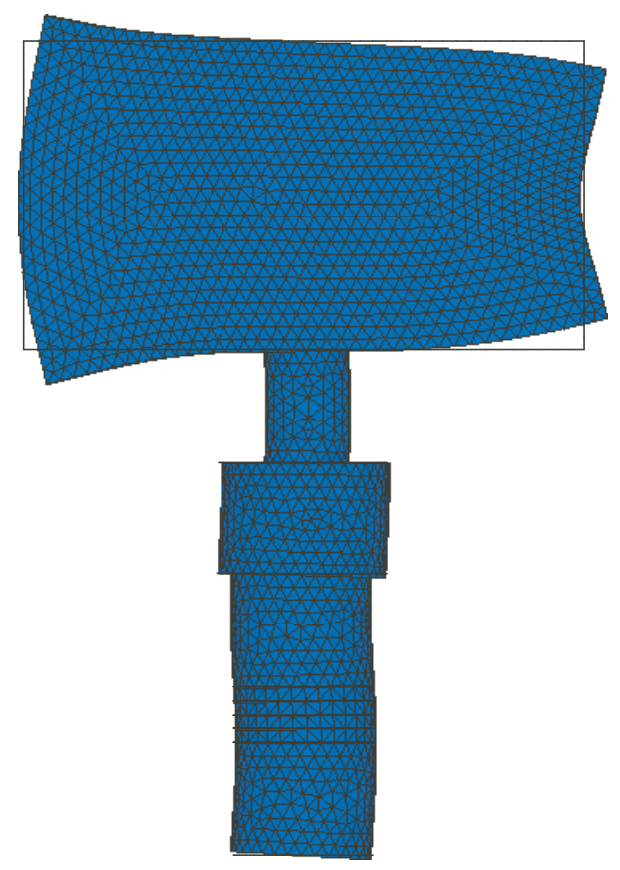

(c)

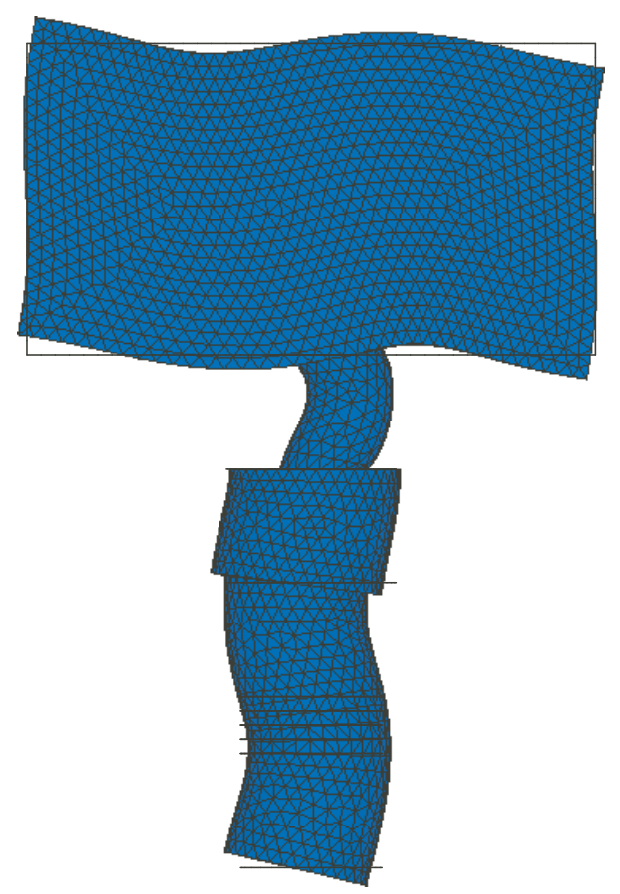

(b)

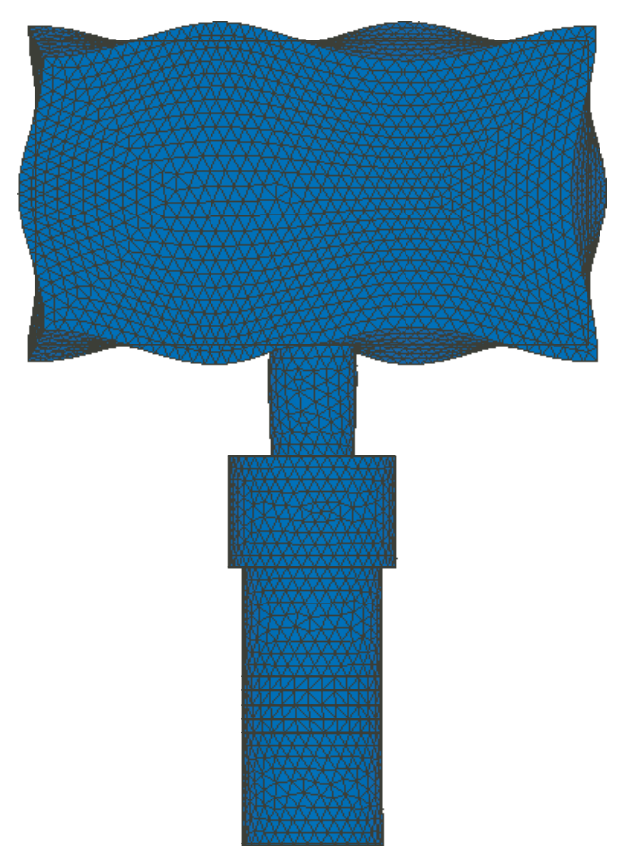

(d)

Figura B.5: Exemplos de modos não-simétricos e modos em outros planos da estrutura. 


\section{Referências}

ADACHI, K.; UEHA, S. Modal vibration control of large ultrasonic tools with the use of wave-trapped horns. Journal Of The Acoustical Society Of America, v. 87, n. 1, p. 208-214, jan. 1990.

ALLIK, H.; HUGHES, T. J. R. Finite element method for piezoelectric vibration. International Journal for Numerical Methods in Engineering, v. 2, n. 2, p. 151-157, 1970.

ARNOLD, F. J.; MUHLEN, S. S. The mechanical pre-stressing in ultrasonic piezotransducers. Ultrasonics, Elsevier Science Bv, v. 39, n. 1, p. 7-11, jan. 2001.

ARNOLD, F. J.; MUHLEN, S. S. The resonance frequencies on mechanically pre-stressed ultrasonic piezotransducers. Ultrasonics, Elsevier Science Bv, v. 39, n. 1, p. 1-5, jan. 2001.

BANGTSSON, E.; NORELAND, D.; BERGGREN, M. Shape optimization of an acoustic horn. Computer Methods In Applied Mechanics And Engineering, Elsevier Science Sa, v. 192, n. 11-12, p. 1533-1571, 2003.

BATHE, K. J. Finite Elements Procedures. New York, USA: Prentice Hall, 1995.

BENDSøE, M. P. Optimal shape design as a material distribution problem. Structural Optimization, v. 1, p. 192-202, 1989.

BENDSøE, M. P.; KIKICHI, N. Generating optimal topologies in structural design using a homogenization method. Computer Methods In Applied Mechanics And Engineering, Elsevier Science Sa Lausanne, v. 71, n. 2, p. 197-224, nov. 1988.

BENDSøE, M. P.; SIGMUND, O. Material interpolation schemes in topology optimization. Archive Of Applied Mechanics, Springer Verlag, v. 69, n. 9-10, p. 635-654, nov. 1999.

BENDSøE, M. P.; SIGMUND, O. Topology Optimization - Theory, Methods and Applications. New York, EUA: Springer, 2003.

BOURDIN, B. Filters in topology optimization. International Journal for Numerial Methods in Engineering, v. 50, n. 9, p. 2143-2158, mar. 2001.

BRISSAUD, M. Characterization of piezoceramics. IEEE Transactions on Ultrasonics, Ferroeletrics and Frequency control, v. 38-6, p. 603-617, 1991.

BRUYNEEL, M.; DUYSINX, P.; FLEURY, C. A family of mma approximations for structural optimization. Structural and Multidisciplinary Optimization, v. 24, n. 4, p. 263-276, out. 2002. 
CARBOnARI, R. C.; SILVA, E. C. N.; NISHIWAKI, S. Design of piezoelectric multi-actuated microtools using topology optimization. Smart Materials \& Structures, v. 14 , n. 6 , p. 1431-1447, dez. 2005.

CARDONI, A.; LUCAS, M. Enhanced vibration performance of ultrasonic block horns. Ultrasonics, Elsevier Science Bv, v. 40, n. 1-8, p. 365-369, maio 2002.

CARDONI, A.; LUCAS, M.; CARTMELL, M.; LIM, F. A novel multiple blade ultrasonic cutting device. Ultrasonics, Elsevier Science Bv, v. 42, n. 1-9, p. 69-74, abr. 2004.

CARDOSO, E. L.; FONSECA, J. S. O. Intermediate density reduction and complexity control in the topology optimization. In: Iberian Latin-American Congress on Computational Methods in Engineering - CILAMCE. [S.l.: s.n.], 1999. v. 20.

CHALLANDE, P. Optimizing ultrasonic transducers based on piezelectric composites using a finite-element method. IEEE Transactions on Ultrasonics, Ferroeletrics and Frequency control, v. 37 (2), p. 135-140, 1990.

CHENG, K. T.; OLHOFF, N. An investigation concerning optimal-design of solid elastic plates. International Journal of Solids and Structures, v. 17, n. 3, p. 305-323, 1981.

CHENG, K. T.; OLHOFF, N. Regularized formulation for optimal-design of axisymmetric plates. International Journal of Solids and Structures, v. 18, n. 2, p. 153-169, 1982.

COOK, R. D.; MALKUS, D. S.; PLESHA, M. E. Concepts and applications of finite element analysis. New York: John Wiley \& Sons, 1989.

DAMJANOVIC, D. Ferroelectric, dielectric and piezoelectric properties of ferroelectric thin films and ceramics. Prog. Phys., v. 61, n. 3, p. 1267-1324, 1998.

DANTZIG, G. Linear Programming and Extensions. [S.l.]: Princeton, New Jersey, 1963.

DESILETS, C.; WOJCIK, G.; NIKODYM, L.; MESTERTON, K. Analyses and measurements of acoustically matched, air-coupled tonpilz transducers. IEEE Ultrasonics Symposium, v. 1, p. 1045-1048, 1999.

DIAZ, A.; SIGMUND, O. Checkerboard patterns in layout optimization. Structural Optimization, v. 10, n. 1, p. 40-45, ago. 1995.

DIAZ, A. R.; BENDSøE, M. P. Shape optimization of structures for multiple loading conditions using a homogenization method. Structural Optimization, v. 4, n. 1, p. 17-22, mar. 1992.

DOGAN, A.; UCHINO, K.; NEWNHAM, R. E. Composite piezoelectric transducer with truncated conical endcaps cymbal. IEEE Transactions on Ultrasonics Ferroelectrics and Frequency Control, v. 44-3, p. 597-605, 1997.

ENSMINGER, D. Ultrasonics: Fundamentals, Technology, Applications. 2. ed. New York, USA: [s.n.], 1988.

EWINS, D. Modal Testing: Theory and Practice. [S.l.]: Brüel \& Kjær, 1986. 
FUKUSHIMA, J.; SUZUKI, K.; KIKUCHI, N. Applications to car bodies - Generalized layout design of three-dimensional shells. In: Optimization of large structural systems; Proceedings of the NATO/DFG Advanced Study Institute, Berchtesgaden, Germany, Sept. 23-Oct. 4, 1991. Vol. 1 (A93-54501 24-39), p. 177-191. [S.l.: s.n.], 1993. p. $177-191$.

GRAHAM, G.; PETZING, J. N.; LUCAS, M. Modal analysis of ultrasonic block horns by espi. Ultrasonics, Elsevier Science Bv, v. 37, n. 2, p. 149-157, fev. 1999.

GUEDES, J. M.; KIKUCHI, N. Preprocessing and postprocessing for materials based on the homogenization method with adaptive finite-element methods. Computer Methods in Applied Mechanics and Engineering, v. 83, n. 2, p. 143-198, out. 1990.

GUEST, J. K.; PREVOST, J. H.; BELYTSCHKO, T. Achieving minimum length scale in topology optimization using nodal design variables and projection functions. International Journal For Numerical Methods In Engineering, v. 61, n. 2, p. 238-254, set. 2004.

GUO, N.; CAWLEY, P.; HITCHINGS, D. The finite-element analysis of the vibration characteristics of piezoelectric disks. Journal Of Sound And Vibration, Academic Press Ltd, v. 159, n. 1, p. 115-138, nov. 1992.

HAFTKA, R. T.; GURDAL, Z.; KAMAT, M. P. Elements of Structural Optimization. 2. ed. [S.l.]: Kluwer Academic Publishers, 1990.

HANSEN, L. V. Topology optimization of free vibrations of fiber laser packages. Structural And Multidisciplinary Optimization, Springer, v. 29, n. 5, p. 341-348, maio 2005.

HASSANI, B.; HINTON, E. A review of homogenization and topology optimization i homogenization theory for media with periodic structure. Computers $\&$ Structures, v. 69, n. 6, p. 707-717, dez. 1998.

HASSANI, B.; HINTON, E. A review of homogenization and topology optimization iii topology optimization using optimality criteria. Computers 65 Structures, v. 69, n. 6, p. 739-756, dez. 1998.

HE, J.-H. Hamilton principle and generalized variational principles of linear thermopiezoelectricity. Journal of Applied Mechanics, ASME, v. 68, n. 4, p. 666-667, 2001. Disponível em: <http://link.aip.org/link/?AMJ/68/666/1>.

HEIKKOLA, E.; LAITINEN, M. Model-based optimization of ultrasonic transducers. Ultrasonics Sonochemistry, Elsevier Science Bv, v. 12, n. 1-2, p. 53-57, jan. 2005.

HIROSE, S.; AOYAGI, M.; TOMIKAWA, Y.; TAKAHASHI, S.; UCHINO, K. High power characteristics at antiresonance frequency of piezoelectric transducers. Ultrasonics, v. 34, n. 2-5, p. 213-217, jun. 1996.

IKEDA, T. Fundamentals of Piezoelectricity. [S.l.]: Oxford University Press, 1996.

JOG, C. S.; HABER, R. B. Stability of finite element models for distributed-parameter optimization and topology design. Computer Methods in Applied Mechanics and Engineering, v. 130, p. 203-226, 1996. 
JOG, C. S.; HABER, R. B.; BENDSØE, M. P. Topology design with optimized, self-adaptive materials. International Journal for Numerical Methods in Engineering, v. 37, n. 8, p. 1323-1350, abr. 1994.

KIKUCHI, N.; HOLLISTER, S.; YOO, J. A concept of image-based integrational cae for production engineering. In: Int. Symp. Optimization and Innovative Design. [S.l.: s.n.], 1997.

KIM, T. S.; KIM, Y. Y. Mac-based mode-tracking in structural topology optimization. Computers 8 Structures, v. 74, n. 3, p. 375-383, jan. 2000.

KOHN, R. V.; STRANG, G. Optimal-design and relaxation of variational-problems .1. Communications on Pure and Applied Mathematics, v. 39, n. 1, p. 113-137, jan. 1986.

KOHN, R. V.; STRANG, G. Optimal-design and relaxation of variational-problems .2. Communications on Pure and Applied Mathematics, v. 39, n. 2, p. 139-182, mar. 1986.

KOHN, R. V.; STRANG, G. Optimal-design and relaxation of variational-problems .3. Communications on Pure and Applied Mathematics, v. 39, n. 3, p. 353-377, maio 1986.

LEHOUCQ, R.; SORENSEN, D.; YANG, C. ARPACK User's Guide: Solution of Large-Scale Eigenvalue Problems with Implicity Restarted Arnoldi Methods. [S.l.]: SIAM, 1998.

LERCH, R. Simulation of piezoelectric devices by 2-dimensional and 3-dimensional finite-elements. Ieee Transactions On Ultrasonics Ferroelectrics And Frequency Control, Ieee-Inst Electrical Electronics Engineers Inc, v. 37, n. 3, p. 233-247, maio 1990.

LUCAS, M.; GRAHAM, G.; SMITH, A. C. Enhanced vibration control of an ultrasonic cutting process. Ultrasonics, Butterworth-Heinemann Ltd, v. 34, n. 2-5, p. 205-211, jun. 1996.

LURIE, K. A.; CHERKAEV, A. V.; FEDOROV, A. V. Regularization of optimal-design problems for bars and plates .1. Journal of Optimization Theory and Applications, v. 37, n. 4, p. 499-522, 1982.

LURIE, K. A.; CHERKAEV, A. V.; FEDOROV, A. V. Regularization of optimal-design problems for bars and plates .2. Journal of Optimization Theory and Applications, v. 37, n. 4, p. 523-543, 1982.

MA, Z. D.; KIKUCHI, N. A new method of sequential approximate optimization for structural optimization problems. Engieneering Optimization, v. 25, n. 3, p. 231-253, 1995.

MAEDA, Y.; NISHIWAKI, S.; IZUI, K.; YOSHIMURA, M.; MATSUI, K.; TERADA, K. Structural topology optimization of vibrating structures with specified eigenfrequencies and eigenmode shapes. International Journal for Numerical Methods in Engineering, v. 67 , n. 5 , p. 597-628, jul. 2006.

MATSUI, K.; TERADA, K. Continuous approximation of material distribution for topology optimization. International Journal For Numerical Methods In Engineering, John Wiley \& Sons Ltd, v. 59, n. 14, p. 1925-1944, abr. 2004. 
MIN, S.; KIKUCHI, N.; PARK, Y. C.; KIM, S.; CHANG, S. Optimal topology design of structures under dynamic loads. Structural Optimization, v. 17, n. 2-3, p. 208-218, abr. 1999.

MOFFETT, M. B.; POWERS, J. M.; JEVNAGER, M. D. A tonpilz projector for use in an underwater horn. Journal Of The Acoustical Society Of America, Amer Inst Physics, v. 103, n. 6, p. 3353-3361, jun. 1998.

MOROZOV, A. K.; WEBB, D. C. A sound projector for acoustic tomography and global ocean monitoring. Ieee Journal Of Oceanic Engineering, Ieee-Inst Electrical Electronics Engineers Inc, v. 28, n. 2, p. 174-185, abr. 2003.

NAILLON, M.; COURSANT, R. H.; BESNIER, F. Analysis of piezoelectric structures by a finite element method. Acta Eletronica, v. 25(4), p. 341-362, 1983.

NEVES, M. M.; RODRIGUES, H.; GUEDES, J. M. Generalized topology design of structures with a buckling load criterion. Structural Optimization, v. 10, n. 2, p. 71-78, out. 1995.

NEWNHAM, R.; DOGAN, A.; XU, Q.; ONITSUKA, K.; TRESSLER, J.; YOSHIKAWA, S. Flextensional moonie actuators. In: Ultrasonics Symposium, 1993. Proceedings., IEEE 1993. [S.l.: s.n.], 1993. p. 509-513 vol.1.

NISSE, E. E. On variational techniques for piezoelectric device analysis. Proceedings of the IEEE, v. 55, n. 8, p. 1524-1525, 1967. ISSN 0018-9219.

NYE, J. F. Physical Properties of Crystals. [S.l.]: Oxford University Press, 1985.

ONITSUKA, K.; DOGAN, A.; TRESSLER, J. F.; XU, Q.; YOSHIKAWA, S.; NEWNHAM, R. E. Metal-ceramic composite transducer, the moonie. Journal of Intelligent Material Systems and Structures, v. 6, p. 447-455, 1995.

OSTERGAARD, D. F.; PAWLAK, T. P. Three-dimensional finite elements for analyzing piezoelectric structures. In: IEEE Ultrasonics Symposim. [S.l.: s.n.], 1986.

PARRINI, L. Design of advanced ultrasonic transducers for welding devices. Ieee Transactions On Ultrasonics Ferroelectrics And Frequency Control, Ieee-Inst Electrical Electronics Engineers Inc, v. 48, n. 6, p. 1632-1639, nov. 2001.

PARRINI, L. New techniques for the design of advanced ultrasonic transducers for wire bonding. Ieee Transactions On Electronics Packaging Manufacturing, Ieee-Inst Electrical Electronics Engineers Inc, v. 26, n. 1, p. 37-45, jan. 2003.

PEDERSEN, N. L. Maximization of eigenvalues using topology optimization. Structural And Multidisciplinary Optimization, Springer-Verlag, v. 20, n. 1, p. 2-11, ago. 2000.

RAHMATALLA, S.; SWAN, C.; ASCE, M. Form finding of sparse structures with continuum topology optimization. Journal of Structural Engineering - ASCE, p. 1707-1716, 2003.

RAHMATALLA, S. F.; SWAN, C. C. A q4/q4 continuum structural topology optimization implementation. Structural And Multidisciplinary Optimization, Springer-Verlag, v. 27, n. 1-2, p. 130-135, maio 2004. 
ROZVANY, G. I. N.; OLHOFF, N.; CHENG, K. T.; TAYLOR, J. E. On the solid plate paradox in structural optimization. Journal of Structural Mechanics, v. 10, n. 1, p. 1-32, 1982.

SHERRIT, S.; BAO, X.; CHANG, Z.; DOLGIN, B. P.; BAR-COHEN, Y.; PAL, D.; KROH, J.; PETERSON, T. Modeling of the ultrasonic/sonic driller/corer: Usdc. IEEE Ultrasonic Symposium, v. 1, p. 4, 2000.

SHUYU, L. Design of piezoelectric sandwich ultrasonic trasnducers with large cross-section. Applied Acoustics, v. 44, p. 249-257, 1995.

SHUYU, L. Sandwiched piezoelectric ultrasonic transducers of longitudinal-torsional compound vibrational modes. IEE Transactions on Ultrasonics, Ferroelectrics, and Frequency Control, v. 44, n. 6, p. 1189-1197, 1997.

SHUYU, L. Optimization of the performance of the sandwich piezoelectric ultrasonic transducer. Journal of Acoustical Society of America, v. 115, n. 1, p. 182-186, 2004.

SHUYU, L. Analysis of the sandwich piezoelectric ultrasonic transducer in coupled vibration. Journal of Acoustical Society of America, v. 117, n. 2, p. 653-661, 2005.

SIGMUND, O. On the design of compliant mechanisms using topology optimization. Mechanics of Structures and Machines, v. 25, n. 4, p. 493-524, 1997.

SIGMUND, O.; PETERSSON, J. Numerical instabilities in topology optimization: A survey on procedures dealing with checkerboards, mesh-dependencies and local minima. 1998. 68-75 p. Disponível em: <http://dx.doi.org/10.1007/BF01214002>.

SILVA, E. Topology optimization applied to the design of linear piezoelectric motors. Journal of Intelligent Material Systems and Structures, v. 14, n. 4/5, p. 309-322, 2003.

SILVA, E. C. N.; FONSECA, J. S. O.; ESPINOSA, F. M.; CRUMM, A. T.; BRADY, G. A.; HALLORAN, J. W.; KIKUCHI, N. Design of piezocomposite materials and piezoelectric transducers using topology optimization - part i. Archives of Computational Methods in Engineering, v. 6, n. 2, p. 117-182, 1999.

SILVA, E. C. N.; KIKUCHI, N. Design of piezoelectric transducers using topology optimization. Smart Materials \& Structures, v. 8, n. 3, p. 350-364, jun. 1999.

SILVA, J. B. D. Análise Numérica de um Transdutor Piezelétrico de Potência para Processamento de Termoplásticos Têxteis. Dissertação (Mestrado) - Escola Politécnica da USP, São Paulo - Brasil, 2006.

STOLPE, M.; SVANBERG, K. An alternative interpolation scheme for minimum compliance topology optimization. Structural and Multidisciplinary Optimization, v. 22, n. 2 , p. $116-124$, set. 2001.

STRANG, G.; KOHN, R. V. Optimal-design in elasticity and plasticity. International Journal for Numerical Methods in Engineering, v. 22, n. 1, p. 183-188, jan. 1986.

SUZUKI, K.; KIKUCHI, N. A homogenization method for shape and topology optimization. Computer Methods in Applied Mechanics and Engineering, v. 93, n. 3, p. 291-318, December 1991. 
SVANBERG, K. The method of moving asymptotesa new method for structural optimization. International Journal for Numerical Methods in Engineering, v. 24, n. 2, p. 359-373, fev. 1987.

SWAN, C. C.; KOSAKA, I. Voigt-reuss topology optimization for structures with linear elastic material behaviours. International Journal for Numerical Methods in Engineering, v. 40, n. 16, p. 3033-3057, 1997.

THOMSEN, J. Topology optimization of structures composed of one or 2 materials. Structural Optimization, v. 5, n. 1-2, p. 108-115, dez. 1992.

TIERSTEN, H. Hamilton's principle for linear piezoelectric media. Proceedings of the IEEE, v. 55, n. 8, p. 1523-1524, 1967. ISSN 0018-9219.

TIMOSHENKO, S.; GOODIER, J. N. Theory of Elasticity. 3. ed. [S.l.: s.n.], 1970.

TURTELTAUB, S. Optimal material properties for transient problems. Structural and Multidisciplinary Optimization, v. 22, n. 2, p. 157-166, set. 2001.

VANDERPLAATS, G. N. Numerical Optimization Techniques for Engineering Design: with Applications. [S.l.]: McGraw-Hill, New York, EUA, 1984.

XU, Q.; YOSHIKAWA, S.; BELSICK, J.; NEWNHAM, R. Piezoelectric composites with high sensitivity and high capacitance for use at high pressures. Ultrasonics, Ferroelectrics and Frequency Control, IEEE Transactions on, v. 38, n. 6, p. 634-639, 1991. ISSN 0885-3010.

YANG R.J., C. C. Optimal topology design using linear programming. Computer and Structures, v. 52, n. 2, p. 265-275, 1994.

YONG, Y. K. A new storage scheme for the lanczos solution of large scale finite element models of piezoelectric resonators. IEEE Ultrasonics Symposium, v. 1, p. 1633-1636, 1995 .

YONG, Y. K.; CHO, Y. Algorithms for eigenvalue problems in piezoelectric finite element analysis. IEEE Ultrasonics Symposium, v. 1, p. 1057-1062, 1994.

YONG, Y. K.; CHO, Y. Numerical algorithms for solutions of large eigenvalue problems in piezoelectric resonators. International Journal For Numerical Methods In Engineering, v. 39, n. 6, p. 909-922, mar. 1996. 


\section{Apêndice A - Piezeletricidade}

A piezeletricidade é uma propriedade que certos materiais apresentam relacionando variáveis mecânicas, como tensão mecânica e deformação, e variáveis elétricas, como campo elétrico e deslocamento elétrico. Pode-se também, definir a piezeletricidade como a polarização elétrica produzida por uma deformação mecânica em certos cristais, ou seja, quando o material piezelétrico é submetido a uma deformação mecânica, um campo elétrico é gerado. O efeito inverso também se aplica, sendo gerada uma deformação mecânica no material piezelétrico quando submetido a um campo elétrico.

\section{A.1 Material Piezelétrico}

O efeito piezelétrico ocorre naturalmente em certos cristais, como o quartzo, no entanto, de maneira muito fraca. Por este motivo, desenvolveu-se novos materiais piezelétricos, como o Titanato Zircanato de Chumbo (PZT), no qual o efeito piezelétrico é induzido artificialmente através de um processo de polarização.

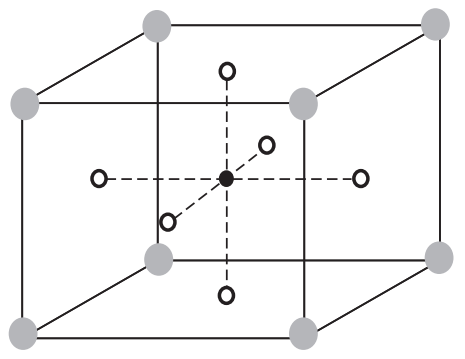

(a)

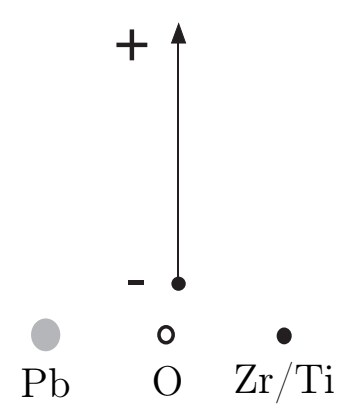

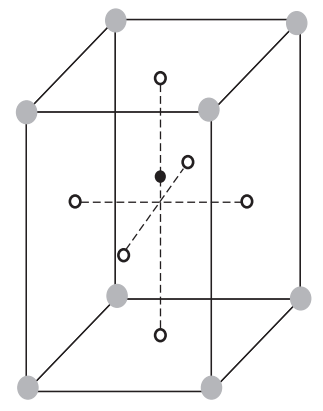

(b)

Figura A.1: Estrutura do PZT antes (a) e depois (b) do processo de polarização.

No PZT existem dipolos elétricos em seu interior (chamados de domínio de Weiss), orientados aleatoriamente antes da polarização (Fig. A.2(a)). Neste estado o material 
não é piezelétrico. A polarização consiste no aquecimento da cerâmica até a temperatura Curie, que é uma temperatura limite acima da qual as propriedades piezelétricas são perdidas.

Em seguida, um intenso campo elétrico $(>2000 \mathrm{~V} / \mathrm{mm})$ é aplicado, o que faz com que os dipolos se alinhem (Fig. A.2(b)), determinando a orientação das propriedades piezelétricas. Com a aplicação do campo, o material se expande na direção axial ao campo e contrai na direção perpendicular a ele. Mantendo o campo e resfriando o material lentamente, obtém-se finalmente um material piezelétrico, cujos dipolos se mantêm alinhados mesmo após retirar o campo elétrico (Fig. A.2(c)). Por causa desta característica, o PZT é um material ferroelétrico, em analogia aos materiais ferromagnéticos.

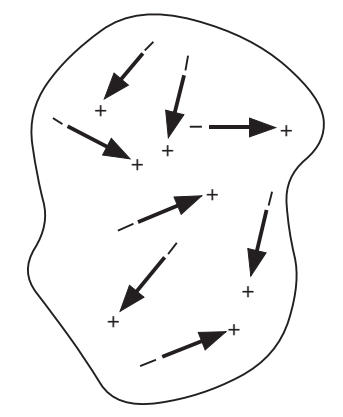

(a)

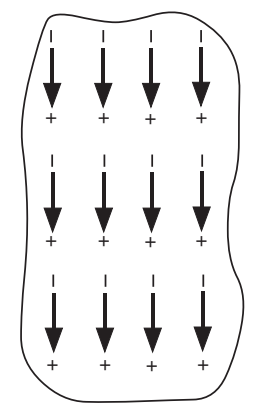

(b)

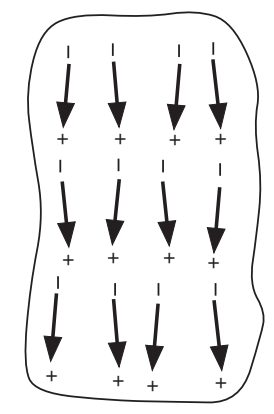

(c)

Figura A.2: Dipolos elétricos no domínio de Weiss: (a) despolarizado; (b) durante polarização; (c) após polarização.

Ao contrário dos cristais piezelétricos como o quartzo, as cerâmicas piezelétricas, como o PZT, possuem uma versatilidade maior, ou seja, suas características físicas, químicas e piezelétricas podem ser ajustadas conforme a aplicação, podendo também, ser confeccionadas em uma infinidade de formatos, por causa do processo de fabricação por aglomeração de partículas.

\section{A.2 Equações Constitutivas Piezelétricas}

As equações constitutivas que definem o comportamento de um material piezelétrico linear são dadas por (IKEDA, 1996; LERCH, 1990):

$$
\begin{aligned}
& \mathbf{T}=\mathbf{c}^{E} \mathbf{S}-\mathbf{e}^{t} \mathbf{E} \\
& \mathbf{D}=\mathbf{e} \mathbf{S}+\varepsilon^{S} \mathbf{E}
\end{aligned}
$$


Os tensores T, S, D e E são dados por:

$$
\mathbf{T}=\left\{\begin{array}{c}
T_{11} \\
T_{22} \\
T_{33} \\
T_{23} \\
T_{13} \\
T_{12}
\end{array}\right\} ; \mathbf{S}=\left\{\begin{array}{c}
S_{11} \\
S_{22} \\
S_{33} \\
S_{23} \\
S_{13} \\
S_{12}
\end{array}\right\} ; \mathbf{D}=\left\{\begin{array}{c}
D_{1} \\
D_{2} \\
D_{3}
\end{array}\right\} ; \mathbf{E}=\left\{\begin{array}{c}
E_{1} \\
E_{2} \\
E_{3}
\end{array}\right\} ; \boldsymbol{u}=\left\{\begin{array}{l}
u_{1} \\
u_{2} \\
u_{3}
\end{array}\right\}
$$

onde $\mathbf{T}$ e $\mathbf{S}$ são os tensores de tensão e de deformação mecânica, respectivamente, em que $S_{i j}=\frac{1}{2}\left(\frac{\partial u_{i}}{\partial x_{j}}+\frac{\partial u_{j}}{\partial x_{i}}\right) ; i, j=1,2,3 . \mathbf{D}, \mathbf{E}$ e $\boldsymbol{u}$ são os vetores de deslocamento elétrico, campo elétrico e deslocamento mecânico, respectivamente. Os índices 1, 2 e 3 indicam as coordenadas $x, y$ e $z$, respectivamente. As matrizes $\mathbf{c}^{E}$, e e $\varepsilon^{S}$ obtidas para a classe de simetria $6 \mathrm{~mm}$ são dadas por:

$$
\begin{gathered}
\mathbf{c}^{E}=\left[\begin{array}{cccccc}
c_{11}^{E} & c_{12}^{E} & c_{13}^{E} & 0 & 0 & 0 \\
c_{12}^{E} & c_{22}^{E} & c_{13}^{E} & 0 & 0 & 0 \\
c_{13}^{E} & c_{13}^{E} & c_{33}^{E} & 0 & 0 & 0 \\
0 & 0 & 0 & c_{44}^{E} & 0 & 0 \\
0 & 0 & 0 & 0 & c_{44}^{E} & 0 \\
0 & 0 & 0 & 0 & 0 & c_{66}^{E}
\end{array}\right] ; \mathbf{e}=\left[\begin{array}{cccccc}
0 & 0 & 0 & 0 & e_{15} & 0 \\
0 & 0 & 0 & e_{15} & 0 & 0 \\
e_{31} & e_{31} & e_{33} & 0 & 0 & 0
\end{array}\right] \\
\varepsilon^{S}=\left[\begin{array}{ccc}
\varepsilon_{11}^{S} & 0 & 0 \\
0 & \varepsilon_{11}^{S} & 0 \\
0 & 0 & \varepsilon_{33}^{S}
\end{array}\right]
\end{gathered}
$$

onde $\mathbf{c}^{E}$, e e $\varepsilon^{S}$ são as matrizes de propriedades de rigidez elástica, piezelétricas e dielétricas, respectivamente. A classe de simetria $6 \mathrm{~mm}$ se baseia na simetria em relação aos eixos dispostos como no sistema de coordenadas hexagonais apresentado na Fig. A.3.

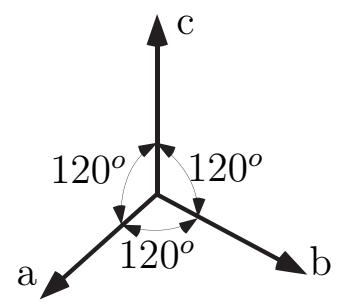

Figura A.3: Sistema de coordenadas hexagonais. 
Substituindo essas matrizes na Eq. (A.1), temos:

$$
\left\{\begin{array}{c}
T_{11} \\
T_{22} \\
T_{33} \\
T_{23} \\
T_{13} \\
T_{12} \\
D_{1} \\
D_{2} \\
D_{3}
\end{array}\right\}=\left[\begin{array}{ccccccccc}
c_{11}^{E} & c_{12}^{E} & c_{13}^{E} & 0 & 0 & 0 & 0 & 0 & e_{31} \\
c_{12}^{E} & c_{22}^{E} & c_{13}^{E} & 0 & 0 & 0 & 0 & 0 & e_{31} \\
c_{13}^{E} & c_{13}^{E} & c_{33}^{E} & 0 & 0 & 0 & 0 & 0 & e_{33} \\
0 & 0 & 0 & c_{44}^{E} & 0 & 0 & 0 & e_{15} & 0 \\
0 & 0 & 0 & 0 & c_{44}^{E} & 0 & e_{15} & 0 & 0 \\
0 & 0 & 0 & 0 & 0 & c_{66}^{E} & 0 & 0 & 0 \\
0 & 0 & 0 & 0 & e_{15} & 0 & -\varepsilon_{11}^{S} & 0 & 0 \\
0 & 0 & 0 & e_{15} & 0 & 0 & 0 & -\varepsilon_{11}^{S} & 0 \\
e_{31} & e_{31} & e_{33} & 0 & 0 & 0 & 0 & 0 & -\varepsilon_{33}^{S}
\end{array}\right]\left\{\begin{array}{c}
S_{11} \\
S_{22} \\
S_{33} \\
S_{23} \\
S_{13} \\
S_{12} \\
-E_{1} \\
-E_{2} \\
-E_{3}
\end{array}\right\}
$$

Os valores das propriedades elásticas, piezelétricas e dielétricas podem ser obtidos na literatura científica da área (IKEDA, 1996). 


\section{Apêndice B - Princípio Variacional Piezelétrico}

O princípio variacional piezelétrico linear é utilizado para obter as equações de equilíbrio piezelétrico, e é descrito nesta seção através do princípio de Hamilton (ALLIK; HUGHES, 1970; NAILLON; COURSANT; BESNIER, 1983; LERCH, 1990), considerando o meio piezelétrico linear.

As equações dinâmicas do meio piezelétrico contínuo são derivadas do princípio de Hamilton, no qual é utilizada a formulação Lagrangeana e o princípio dos trabalhos virtuais adaptados para incluir a contribuição elétrica ao sistema mecânico. Essas equações dinâmicas do meio piezelétrico contínuo são dadas por (NISSE, 1967; TIERSTEN, 1967; ALLIK; HUGHES, 1970; HE, 2001):

$$
\delta \int_{t_{1}}^{t_{2}}(\mathcal{L}+\mathcal{W}) d t=0
$$

onde $t_{1}$ e $t_{2}$ definem o intervalo de tempo (pela definição do princípio dos trabalhos virtuais sabe-se que $t_{1}=$ e $\left.t_{2}=t\right), \mathcal{L}$ é o Lagrangeano e $\mathcal{W}$ é o trabalho virtual dos carregamentos

mecânicos e elétricos das forças externas. O Lagrangeano $\mathcal{L}$ é definido pela soma das energias cinéticas $\mathcal{T}$ e da entalpia elétrica $\mathcal{H}$, como mostrado na expressão abaixo (NISSE, 1967; TIERSTEN, 1967; HE, 2001):

$$
\mathcal{L}=\mathcal{T}+\mathcal{H}
$$

sendo, a entalpia elétrica $\mathcal{H}$ obtida do funcional de Gibbs $(\mathcal{G}=\mathcal{H})$ (NYE, 1985; IKEDA, 1996; DAMJANOVIC, 1998), considerando somente o efeito piezelétrico linear.

Da teoria piezelétrica linear, $\mathcal{H}$ é dado por:

$$
\mathcal{H}=\frac{1}{2} c_{i j k l}^{E} \sigma_{i j} \sigma_{k l}-e_{k i j} E_{k} S_{i j}-\frac{1}{2} \varepsilon_{i j}^{S} E_{i} E_{j}
$$


Das equações constitutivas piezelétricas (Eq. (A.1)) pode-se escrever:

$$
\begin{aligned}
& \{\mathbf{T}\}=\left[\mathbf{c}^{E}\right]\{\mathbf{S}\}+[\mathbf{e}]^{t}\{-\mathbf{E}\} \\
& \{\mathbf{D}\}=[\mathbf{e}]\{\mathbf{S}\}+\left[-\boldsymbol{\varepsilon}^{S}\right]\{-\mathbf{E}\}
\end{aligned}
$$

Substituindo a Eq. (B.4) na Eq. (B.3), obtém-se:

$$
\mathcal{H}=\frac{1}{2}\left[\{\mathbf{S}\}^{t}\{\mathbf{T}\}-\{\mathbf{E}\}^{t}\{\mathbf{D}\}\right]
$$

e $\mathcal{T}$ é dado por (NAILLON; COURSANT; BESNIER, 1983):

$$
\mathcal{T}=\frac{1}{2} \rho\{\dot{\mathbf{u}}\}^{t}\{\dot{\mathbf{u}}\}
$$

onde $\dot{\mathbf{u}}$ é o campo de velocidades e $\rho$ é a densidade do meio.

O lagrangeano na forma variacional é definido por:

$$
\delta \mathcal{L}=\int_{t_{1}}^{t_{2}}(\delta \mathcal{T}+\delta \mathcal{H}) d t
$$

e, portanto, variacionando $\mathcal{T}$ e $\mathcal{H}$, obtém-se:

$$
\begin{aligned}
\delta \mathcal{T} & =\int_{t_{1}}^{t_{2}} \rho\{\delta \dot{\mathbf{u}}\}^{t}\{\dot{\mathbf{u}}\} d t=\underbrace{\left.\rho\{\delta \mathbf{u}\}^{t}\{\dot{\mathbf{u}}\}\right|_{t_{1}} ^{t_{2}}}_{=0 \rightarrow t_{1}=t_{2}=t}-\int_{t_{1}}^{t_{2}} \rho\{\delta \mathbf{u}\}^{t}\{\ddot{\mathbf{u}}\} d t= \\
& =-\int_{t_{1}}^{t_{2}} \rho\{\delta \mathbf{u}\}^{t}\{\ddot{\mathbf{u}}\} d t
\end{aligned}
$$

e

$$
\delta \mathcal{H}=\int_{t_{1}}^{t_{2}}\left[\{\delta \mathbf{S}\}^{t}\left([\mathbf{c}]^{E}\{\mathbf{S}\}-[\mathbf{e}]^{t}\{\mathbf{E}\}\right)-\{\delta \mathbf{E}\}^{t}\left([\mathbf{e}]\{\mathbf{S}\}+[\varepsilon]^{S}\{\mathbf{E}\}\right)\right] d t
$$

Então, substituindo-se as Eqs. (B.8) e (B.9) na Eq. (B.7), tem-se:

$$
\begin{aligned}
\delta \mathcal{L}= & -\int_{t_{1}}^{t_{2}} \int_{V} \rho\{\delta \mathbf{u}\}^{t}\{\ddot{\mathbf{u}}\} d V d t+ \\
& +\int_{t_{1}}^{t_{2}} \int_{V}\{\delta \mathbf{S}\}^{t}\left([\mathbf{c}]^{E}\{\mathbf{S}\}-[\mathbf{e}]^{t}\{\mathbf{E}\}\right) d V d t- \\
& -\int_{t_{1}}^{t_{2}} \int_{V}\{\delta \mathbf{E}\}^{t}\left([\mathbf{e}]\{\mathbf{S}\}+[\boldsymbol{\varepsilon}]^{S}\{\mathbf{E}\}\right) d V d t
\end{aligned}
$$

O trabalho virtual $\delta \mathcal{W}$ realizado pelos carregamentos mecânicos e elétricos para uma variação arbitrária do campo de deslocamento $\{\delta \mathbf{u}\}$ e do potencial elétrico $\{\delta \phi\}$, respectivamente, é dado por:

$$
\delta W=\int\left(\{\delta \mathbf{u}\}^{t}\{\mathbf{f}\}-\{\delta \phi\}^{t}\{\mathbf{q}\}\right) d t
$$

sendo que $\{\mathbf{f}\}$ é o carregamento (interno e externo) mecânico pontual e $\{\mathbf{q}\}$ é o potencial 
(interno e externo) elétrico pontual. Desta forma, substituindo as Eqs. (B.10) e (B.11) na Eq. (B.11), o variacional resultante é (ALLIK; HUGHES, 1970):

$$
\begin{aligned}
& -\int_{V}\left[\rho\{\delta \mathbf{u}\}^{t}\{\ddot{\mathbf{u}}\}\right] d V- \\
& -\int_{V}\left[\{\delta \mathbf{S}\}^{t}\left([\mathbf{c}]^{E}\{\mathbf{S}\}-[\mathbf{e}]^{t}\{\mathbf{E}\}\right)+\{\delta \mathbf{E}\}^{t}\left([\mathbf{e}]\{\mathbf{S}\}+[\boldsymbol{\varepsilon}]^{S}\{\mathbf{E}\}\right)\right] d V+ \\
& +\{\delta \mathbf{u}\}^{t}\{\mathbf{f}\}-\{\delta \phi\}^{t}\{\mathbf{q}\}=0
\end{aligned}
$$

e portanto, tem-se as equações de equilíbrio piezelétrico:

$$
\begin{aligned}
\int_{V}\left[\rho\{\delta \mathbf{u}\}^{t}\{\ddot{\mathbf{u}}\}\right] d V+ & \int_{V}\{\delta \mathbf{S}\}^{t}\left([\mathbf{c}]^{E}\{\mathbf{S}\}-[\mathbf{e}]^{t}\{\mathbf{E}\}\right) d V=\{\delta \mathbf{u}\}^{t}\{\mathbf{f}\} \\
\int_{V}\{\delta \mathbf{E}\}^{t}\left([\mathbf{e}]\{\mathbf{S}\}+[\boldsymbol{\varepsilon}]^{S}\{\mathbf{E}\}\right) d V & =\{\delta \phi\}^{t}\{\mathbf{q}\}
\end{aligned}
$$




\section{Apêndice C - Implementação do Controle Elétrico por Potencial Elétrico}

\section{C.1 Implementação no MEF}

Considerando a equação do problema elétrico (Eq. (2.59)), pode-se expressá-la da seguinte maneira:

$$
\left[\begin{array}{cc}
\hat{\mathbf{K}}_{\text {prescr }} & \hat{\mathbf{K}}_{\text {prescr rest }} \\
\hat{\mathbf{K}}_{\text {prescr rest }} & \hat{\mathbf{K}}_{\text {rest rest }}
\end{array}\right]\left\{\begin{array}{c}
\phi_{\text {prescr }} \\
\boldsymbol{\Psi}_{\text {rest }}
\end{array}\right\}=\left\{\begin{array}{c}
\mathbf{Q}_{\text {prescr }} \\
\boldsymbol{\Gamma}_{\text {rest }}
\end{array}\right\}
$$

onde $\phi_{\text {prescr }}$ é o vetor de potenciais elétricos prescritos (por exemplo, excitação elétrica de entrada), $\boldsymbol{\Psi}_{\text {rest }}$ são todos os outros termos do vetor deslocamento $\{\boldsymbol{\Psi}\}$ (incluindo deslocamento mecânico e potencial elétrico não-prescrito). $\mathbf{Q}_{\text {prescr }}$ é o vetor de cargas elétricas dos termos referentes aos graus de liberdade dos potenciais elétricos prescritos e $\boldsymbol{\Gamma}_{\text {rest }}$ é o restante do vetor de carregamento $\{\boldsymbol{\Gamma}\}$. Os termos $\hat{\mathbf{K}}_{i j}(i, j=$ prescr, rest $)$ são os termos da matriz $[\hat{\mathbf{K}}]$ referentes aos graus de liberdade dos potenciais elétricos prescritos e do restante da matriz.

Então, quando a excitação é por potencial elétrico, escreve-se a Eq. (C.1) como:

$$
\left[\begin{array}{ll}
\mathbf{I} & 0 \\
0 & \hat{\mathbf{K}}_{\text {rest rest }}
\end{array}\right]\left\{\begin{array}{c}
\boldsymbol{\phi}_{\text {prescr }} \\
\boldsymbol{\Psi}_{\text {rest }}
\end{array}\right\}=\left\{\begin{array}{c}
\boldsymbol{\phi}_{\text {prescr }} \\
\boldsymbol{\Gamma}_{\text {rest }}-\hat{\mathbf{K}}_{\text {rest prescr }} \boldsymbol{\phi}_{\text {prescr }}
\end{array}\right\}
$$

Portanto, os deslocamentos mecânicos e os potenciais elétricos nodais $\left\{\boldsymbol{\Psi}_{\text {rest }}\right\}$ encontram-se solucionando a Eq. (C.2). Note que solucionando esse sistema de equações recuperam-se os potenciais prescritos $\left\{\phi_{\text {prescr }}\right\}$. 


\section{C.2 Análise de Sensibilidades}

Derivando a Eq. (C.2), tem-se:

$$
\begin{gathered}
{\left[\begin{array}{cc}
\frac{\partial \mathbf{I}}{\partial \gamma} & 0 \\
0 & \frac{\partial \hat{\mathbf{K}}_{\text {rest rest }}}{\partial \gamma}
\end{array}\right]\left\{\begin{array}{c}
\boldsymbol{\phi}_{\text {prescr }} \\
\boldsymbol{\Psi}_{\text {rest }}
\end{array}\right\}+\left[\begin{array}{cc}
\mathbf{I} & 0 \\
0 & \hat{\mathbf{K}}_{\text {rest rest }}
\end{array}\right]\left\{\begin{array}{c}
\frac{\partial \boldsymbol{\phi}_{\text {prescr }}}{\partial \gamma} \\
\frac{\partial \mathbf{\Psi}_{\text {rest }}}{\partial \gamma}
\end{array}\right\}=} \\
\frac{\partial \boldsymbol{\phi}_{\text {prescr }}}{\partial \gamma} \\
=\left\{\begin{array}{c}
\left.\frac{\partial \Gamma_{\text {rest }}}{\partial \gamma}-\frac{\partial \hat{\mathbf{K}}_{\text {prescr rest }}}{\partial \gamma} \boldsymbol{\phi}_{\text {prescr }}-\hat{\mathbf{K}}_{\text {prescr rest }} \frac{\partial \boldsymbol{\phi}_{\text {prescr }}}{\partial \gamma}\right\}
\end{array}\right.
\end{gathered}
$$

e como os potenciais elétricos prescritos não dependem da variável de projeto $\left(\frac{\partial \phi_{\text {prescr }}}{\partial \gamma}=\right.$ 0), então a Eq. (C.3) pode ser simplificada:

$$
\begin{aligned}
& {\left[\begin{array}{cc}
0 & 0 \\
0 & \frac{\partial \hat{\mathbf{K}}_{\text {rest rest }}^{\partial \gamma}}{\partial \gamma}
\end{array}\right]\left\{\begin{array}{c}
\boldsymbol{\phi}_{\text {prescr }} \\
\boldsymbol{\Psi}_{\text {rest }}
\end{array}\right\}+\left[\begin{array}{cc}
\mathbf{I} & 0 \\
0 & \hat{\mathbf{K}}_{\text {rest rest }}
\end{array}\right]\left\{\begin{array}{c}
0 \\
\left.\frac{\partial \mathbf{\Psi}_{\text {rest }}}{\partial \gamma}\right\}
\end{array}\right\} } \\
&=\left\{\begin{array}{c}
0 \\
-\frac{\partial \hat{\mathbf{K}}_{\text {prescr rest }}}{\partial \gamma} \boldsymbol{\phi}_{\text {prescr }}
\end{array}\right\}
\end{aligned}
$$

e fazendo:

$$
\left[\begin{array}{cc}
0 & 0 \\
0 & \frac{\partial \hat{\mathbf{K}}_{\text {rest rest }}}{\partial \gamma}
\end{array}\right]\left\{\begin{array}{c}
\boldsymbol{\phi}_{\text {prescr }} \\
\boldsymbol{\Psi}_{\text {rest }}
\end{array}\right\}=\left\{\begin{array}{c}
0 \\
\frac{\partial \hat{\mathbf{K}}_{\text {rest rest }}}{\partial \gamma} \boldsymbol{\Psi}_{\text {rest }}
\end{array}\right\}
$$

pode-se reescrever a Eq. (C.4) da seguinte maneira:

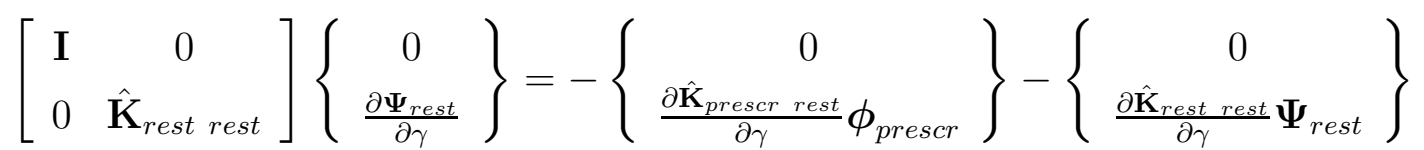

O lado direito da Eq. (C.6) pode ser substituido como a multiplicação de uma matriz e um vetor da seguinte forma:

$$
\begin{gathered}
\left\{\begin{array}{c}
0 \\
\frac{\partial \hat{\mathbf{K}}_{\text {prescr rest }}}{\partial \gamma} \boldsymbol{\phi}_{\text {prescr }}
\end{array}\right\}+\left\{\begin{array}{c}
0 \\
\frac{\partial \hat{\mathbf{K}}_{\text {rest rest }}}{\partial \gamma} \boldsymbol{\Psi}_{\text {rest }}
\end{array}\right\}= \\
=\left[\begin{array}{cc}
0 & 0 \\
\frac{\partial \hat{\mathbf{K}}_{\text {prescr rest }}}{\partial \gamma} & \frac{\partial \hat{\mathbf{K}}_{\text {rest rest }}}{\partial \gamma}
\end{array}\right]\left\{\begin{array}{c}
\boldsymbol{\phi}_{\text {prescr }} \\
\boldsymbol{\Psi}_{\text {rest }}
\end{array}\right\}
\end{gathered}
$$


e fazendo as seguintes simplificações:

$$
\begin{aligned}
& {[\tilde{\mathbf{K}}]=\left[\begin{array}{cc}
\mathbf{I} & 0 \\
0 & \hat{\mathbf{K}}_{\text {rest rest }}
\end{array}\right]} \\
& {\left[\frac{\partial \tilde{\mathbf{K}}}{\partial \gamma}\right]=\left[\begin{array}{cc}
0 & 0 \\
\frac{\partial \hat{\mathbf{K}}_{\text {prescr rest }}}{\partial \gamma} & \frac{\partial \hat{\mathbf{K}}_{\text {rest rest }}}{\partial \gamma}
\end{array}\right]} \\
& \{\boldsymbol{\Psi}\}=\left\{\begin{array}{c}
\boldsymbol{\phi}_{\text {prescr }} \\
\boldsymbol{\Psi}_{\text {rest }}
\end{array}\right\} \\
& \left\{\begin{array}{c}
0 \\
\left.\frac{\partial \tilde{\boldsymbol{\Psi}}}{\partial \gamma}\right\}=\left\{\begin{array}{c}
0 \\
\frac{\partial \boldsymbol{\Psi}_{\text {rest }}}{\partial \gamma}
\end{array}\right\}
\end{array}\right.
\end{aligned}
$$

tem-se a derivada do vetor de deslocamentos mecânicos e potenciais elétricos nodais $(\partial \tilde{\boldsymbol{\Psi}} / \partial \gamma)$ como:

$$
\left\{\frac{\partial \tilde{\boldsymbol{\Psi}}}{\partial \gamma}\right\}=-[\tilde{\mathbf{K}}]^{-1}\left[\frac{\partial \tilde{\mathbf{K}}}{\partial \gamma}\right]\{\boldsymbol{\Psi}\}
$$




\section{Apêndice D - Formulação da Transdução Média}

O conceito de transdução média é utilizado para atender a condição de flexibilidade estrutural ou máxima deformação, através de uma formulação que permite se obter o máximo deslocamento em direções e regiões determinadas, com a aplicação de energia elétrica no meio piezelétrico, cargas elétricas ou potênciais elétricos (voltagem). A transdução média é obtida aplicando-se o teorema da reciprocidade (ou teorema de Betti) proveniente da teoria da elasticidade (TIMOSHENKO; GOODIER, 1970) e estendê-lo para o meio piezelétrico.

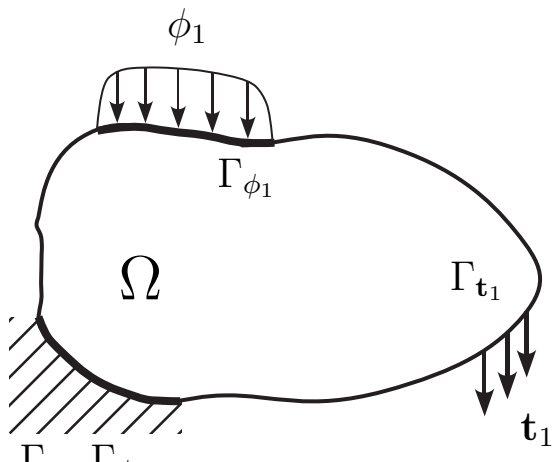

$\Gamma_{\mathbf{u}}, \Gamma_{\phi}$

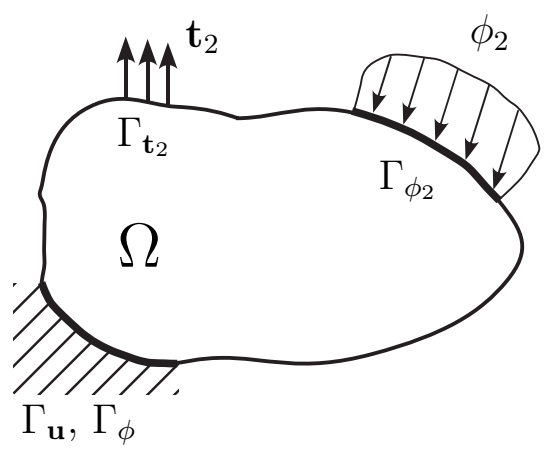

(b) caso b

(a) caso a

Figura D.1: Extensão do teorema da reciprocidade para o meio piezelétrico.

Desta forma, considere um corpo piezelétrico mostrado na Fig. D.1, sujeito a potenciais elétricos $\phi_{1}$ e $\phi_{2}$, aplicados nas regiões $\Gamma_{\phi_{1}}$ e $\Gamma_{\phi_{2}}$ (região denominada de eletrodos da piezocerâmica), respectivamente, e carregamentos mecânicos $\mathbf{t}_{1}$ e $\mathbf{t}_{2}$, aplicados nas regiões $\Gamma_{\mathbf{t}_{1}}$ e $\Gamma_{\mathbf{t}_{2}}$, respectivamente. Primeiro, aplicam-se simultaneamente o potencial elétrico $\phi_{1}$ e o carregamento mecânico $\mathbf{t}_{1}$ nas suas respectivas regiões $\Gamma_{\phi_{1}}$ e $\Gamma_{\mathbf{t}_{1}}$, mantendo-os constantes, Fig. D.1(a), enquanto o potêncial elétrico $\phi_{2}$ e o carregamento mecânico $\mathbf{t}_{2}$ são aplicados simultaneamente nas regiões $\Gamma_{\phi_{2}}$ e $\Gamma_{\mathbf{t}_{2}}$, respectivamente, 
Fig. D.1(b). Portanto, o trabalho total (mecânico e elétrico) realizado pelos potênciais elétricos e carregamentos mecânicos nas regiões é dado pela expressão:

$$
\begin{aligned}
\prod_{1} & =\frac{1}{2} \int_{\Gamma_{\mathbf{t}_{1}}} \mathbf{t}_{1} \mathbf{u}_{1} d \Gamma+\frac{1}{2} \int_{\Gamma_{\phi_{1}}} d_{1} \phi_{1} d \Gamma+\int_{\Gamma_{\mathbf{t}_{1}}} \mathbf{t}_{1} \mathbf{u}_{2} d \Gamma+ \\
& +\int_{\Gamma_{\phi_{1}}} d_{1} \phi_{2} d \Gamma+\frac{1}{2} \int_{\Gamma_{\mathbf{t}_{2}}} \mathbf{t}_{2} \mathbf{u}_{2} d \Gamma+\frac{1}{2} \int_{\Gamma_{\phi_{2}}} d_{2} \phi_{2} d \Gamma
\end{aligned}
$$

onde $d=\mathbf{D} \cdot \mathbf{n}, \mathbf{D}$ é o vetor deslocamento elétrico, e $\mathbf{n}$ é o vetor normal à superfície.

No entanto, aplicando-se simultaneamente o potêncial elétrico $\phi_{2}$ e o carregamento mecânico $\mathbf{t}_{2}$ nas suas respectivas superfícies $\Gamma_{\phi_{2}}$ e $\Gamma_{\mathbf{t}_{2}}$, ao contrário do caso anterior, ou seja, aplicando-se primeiro $\phi_{2}$ e $\mathbf{t}_{2}$, e mantendo-os constantes, enquanto $\phi_{1}$ e $\mathbf{t}_{1}$ são aplicados simultaneamente no meio $\Gamma_{\phi_{1}}$ e $\Gamma_{\mathbf{t}_{1}}$, respectivamente), então o trabalho total (mecânico e elétrico) realizado pelos potênciais elétricos e carregamentos mecânicos nas regiões é dado pela expressão:

$$
\begin{aligned}
\prod_{2} & =\frac{1}{2} \int_{\Gamma_{\mathbf{t}_{2}}} \mathbf{t}_{2} \mathbf{u}_{2} d \Gamma+\frac{1}{2} \int_{\Gamma_{\phi_{2}}} d_{2} \phi_{2} d \Gamma+\int_{\Gamma_{\mathbf{t}_{2}}} \mathbf{t}_{2} \mathbf{u}_{1} d \Gamma+ \\
& +\int_{\Gamma_{\phi_{2}}} d_{2} \phi_{1} d \Gamma+\frac{1}{2} \int_{\Gamma_{\mathbf{t}_{1}}} \mathbf{t}_{1} \mathbf{u}_{1} d \Gamma+\frac{1}{2} \int_{\Gamma_{\phi_{1}}} d_{1} \phi_{1} d \Gamma
\end{aligned}
$$

Em cada caso, desde que o carregamento mecânico e o potêncial elétrico nas regiões sejam aplicados gradativamente, o trabalho feito será armazenado como energia de deformação e elétrica no corpo. Como o estado final para cada caso é idêntico, $\prod_{1}=\prod_{2}$, concluí-se que:

$$
\begin{aligned}
L_{2}\left(\mathbf{u}_{1}, \phi_{1}\right) & =\int_{\Gamma_{\mathbf{t}_{2}}} \mathbf{t}_{2} \mathbf{u}_{1} d \Gamma+\int_{\Gamma_{d_{2}}} d_{2} \phi_{1} d \Gamma= \\
& =\int_{\Gamma_{\mathbf{t}_{1}}} \mathbf{t}_{1} \mathbf{u}_{2} d \Gamma+\int_{\Gamma_{d_{1}}} d_{1} \phi_{2} d \Gamma=L_{1}\left(\mathbf{u}_{2}, \phi_{2}\right)
\end{aligned}
$$

a equação acima é o teorema da reciprocidade estendido para o meio piezelétrico. Analogamente à energia mútua, $L_{1}\left(\mathbf{u}_{2}, \phi_{2}\right)$ ou $L_{2}\left(\mathbf{u}_{1}, \phi_{1}\right)$ são denominadas de transdução média, no qual as quantidade mecânicas e elétricas estão envolvidas, Fig. D.1. Agora considerando a formulação da equação de equilíbrio do meio piezelétrico, temos:

$$
\begin{array}{r}
\int_{\Omega} \rho(\delta \mathbf{u})^{t} \ddot{\mathbf{u}} d \Omega+\int_{\Omega} \varepsilon(\mathbf{u})^{t} \mathbf{c}^{E} \varepsilon(\mathbf{v}) d \Omega+\int_{\Omega}(\nabla \phi)^{t} \mathbf{e}^{t} \varepsilon(\mathbf{v}) d \Omega=\int_{\Gamma_{t}} \mathbf{t v} d \Gamma \\
\int_{\Omega} \varepsilon(\mathbf{u})^{t} \mathbf{e}(\nabla \varphi) d \Omega-\int_{\Omega}(\nabla \phi)^{t} \epsilon^{S}(\nabla \varphi) d \Omega=\int_{\Gamma_{\phi}} d \varphi d \Gamma \\
\text { para } \mathbf{u}, \phi \in V e \forall \mathbf{v}, \forall \phi \in V
\end{array}
$$


onde $V=\left(\mathbf{v}=v_{j} \bar{e}_{j}, \varphi: v_{j}, \phi_{i} \in H^{1}(\Omega) \operatorname{com} \mathbf{v}=0\right.$ em $\Gamma_{\mathbf{u}}$ e $\varphi=0$ em $\Gamma_{\phi}, j=1$ ou 3$), \Omega$ é o domínio do meio piezocerâmico (também pode conter um material não piezelétrico), $\nabla$ é o operador gradiente, $\mathbf{c}^{E}$, e e $\epsilon^{S}$ são as propriedades elásticas a campo elétrico constante, piezelétricas e dielétricas a deformação constante, respectivamente do meio piezelétrico. O índice $j$ assume o valor 1 ou 3, porque o problema está considerando no plano $1-3$. A piezocerâmica está polarizada na direção $\sharp 3$. A Eq. (D.4) pode ser escrita numa forma mais compacta, como mostrado abaixo:

$$
\begin{aligned}
A(\mathbf{u}, \mathbf{u}) & =\int_{\Omega} \rho(\delta \mathbf{u})^{t} \ddot{\mathbf{u}} d \Omega \\
B(\mathbf{u}, \mathbf{v}) & =\int_{\Omega} \varepsilon(\mathbf{u})^{t} \mathbf{c}^{E} \varepsilon(\mathbf{v}) d \Omega \\
C(\phi, \mathbf{v}) & =\int_{\Omega}(\nabla \phi)^{t} \mathbf{e}^{t} \varepsilon(\mathbf{v}) d \Omega \\
D(\phi, \varphi) & =\int_{\Omega}(\nabla \phi)^{t} \epsilon^{S}(\nabla \varphi) d \Omega \\
L_{t}(\mathbf{t}, \mathbf{v}) & =\int_{\Gamma_{t}} \mathbf{t v} d \Gamma \\
L_{d}(d, \varphi) & =\int_{\Gamma_{\phi}} d \varphi d \Gamma
\end{aligned}
$$

Portanto, D.4 torna-se:

$$
\begin{array}{r}
A(\mathbf{u}, \mathbf{u})+B(\mathbf{u}, \mathbf{v})+C(\phi, \mathbf{v})=L_{t}(\mathbf{t}, \mathbf{v}) \\
C(\varphi, \mathbf{u})-D(\phi, \varphi)=L_{d}(d, \varphi)
\end{array}
$$

Fazendo $\mathbf{u}$ e $\mathbf{v}$ iguais à $\mathbf{u}_{1}$ e $\mathbf{u}_{2}, \mathbf{t}$ iguais à $\mathbf{t}_{1}$ e $\mathbf{t}_{2}, \mathrm{~d}$ iguais à $d_{1}$ e $d_{2}$, e finalmente, $\phi$ iguais à $\phi_{1}$ e $\phi_{2}$ (desde que $\mathbf{v}$ e $\phi$ sejam arbritários), obtém-se:

$$
\begin{array}{r}
A\left(\mathbf{u}_{1}, \mathbf{u}_{1}\right)+B\left(\mathbf{u}_{1}, \mathbf{u}_{2}\right)+C\left(\phi_{1}, \mathbf{u}_{2}\right)=L_{t_{1}}\left(\mathbf{t}_{1}, \mathbf{u}_{2}\right) \\
C\left(\phi_{2}, \mathbf{u}_{1}\right)-D\left(\phi_{1}, \phi_{2}\right)=L_{d}\left(d_{1}, \phi_{2}\right)
\end{array}
$$

e:

$$
\begin{array}{r}
A\left(\mathbf{u}_{2}, \mathbf{u}_{2}\right)+B\left(\mathbf{u}_{2}, \mathbf{u}_{1}\right)+C\left(\phi_{2}, \mathbf{u}_{1}\right)=L_{t_{2}}\left(\mathbf{t}_{2}, \mathbf{u}_{1}\right) \\
C\left(\phi_{1}, \mathbf{u}_{2}\right)-D\left(\phi_{2}, \phi_{1}\right)=L_{d}\left(d_{2}, \phi_{1}\right)
\end{array}
$$

Portanto, substituindo as Eqs. (D.7) e (D.8), na equações na Eq. (D.3), resultando 
na expressão abaixo:

$$
\begin{aligned}
L_{2}\left(\mathbf{u}_{1}, \phi_{1}\right) & =A\left(\mathbf{u}_{1}, \mathbf{u}_{1}\right)+B\left(\mathbf{u}_{1}, \mathbf{u}_{2}\right)+C\left(\phi_{1}, \mathbf{u}_{2}\right)+C\left(\phi_{2}, \mathbf{u}_{1}\right)-D\left(\phi_{1}, \phi_{2}\right)= \\
& =A\left(\mathbf{u}_{2}, \mathbf{u}_{2}\right)+B\left(\mathbf{u}_{2}, \mathbf{u}_{1}\right)+C\left(\phi_{2}, \mathbf{u}_{1}\right)+C\left(\phi_{1}, \mathbf{u}_{2}\right)-D\left(\phi_{2}, \phi_{1}\right)= \\
& =L_{1}\left(\mathbf{u}_{2}, \phi_{2}\right)
\end{aligned}
$$

Os operadores definidos na Eq. (D.4), bem como a transdução média (Eq. (D.9)) são operadores auto-adjuntos, ou seja, possuem as propriedades definidas pela seguinte expressão:

$$
(u, \mathbf{L} v)=(v, \mathbf{L} u)
$$

onde:

$$
(u, \mathbf{L} v)=\int_{0}^{L} u \mathbf{L} v d x
$$

